\title{
VARSKIN MOD2 and SADDE MOD2: Computer Codes for Assessing Skin Dose from Skin Contamination
}

Manuscript Completed: November 1992

Date Published: December 1992

Prepared by

J. S. Durham

J. M. Bell, NRC Technical Monitor

Pacific Northwest Laboratory

Richland, WA 99352

\section{Prepared for}

Division of Regulatory Applications

Office of Nuclear Regulatory Research

U.S. Nuclear Regulatory Commission

Washington, DC 20555

NRC FIN L1543

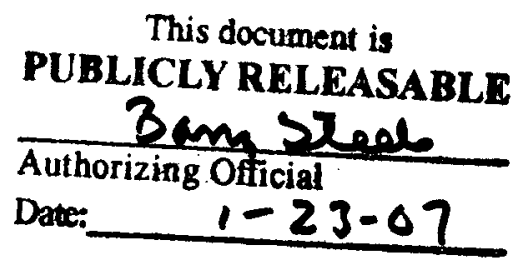




\section{DISCLAIMER}

This report was prepared as an account of work sponsored by an agency of the United States Government. Neither the United States Government nor any agency Thereof, nor any of their employees, makes any warranty, express or implied, or assumes any legal liability or responsibility for the accuracy, completeness, or usefulness of any information, apparatus, product, or process disclosed, or represents that its use would not infringe privately owned rights. Reference herein to any specific commercial product, process, or service by trade name, trademark, manufacturer, or otherwise does not necessarily constitute or imply its endorsement, recommendation, or favoring by the United States Government or any agency thereof. The views and opinions of authors expressed herein do not necessarily state or reflect those of the United States Government or any agency thereof. 


\section{DISCLAIMER}

Portions of this document may be illegible in electronic image products. Images are produced from the best available original document. 


\begin{abstract}
The computer code VARSKIN has been modified to calculate dose to skin from three-dimensional sources, sources separated from the skin by layers of protective clothing, and gamma dose from certain radionuclides. A correction for backscatter has also been incorporated for certain geometries. This document describes the new code, VARSKIN Mod 2, including installation and operation instructions, provides detailed descriptions of the models used, and suggests methods for avoiding misuse of the code. The input data file for VARSKIN Mod 2 has been modified to reflect current physical data, to include the contribution to dose from internal

conversion and Auger electrons, and to reflect a correction for low-energy electrons.

In addition, the computer code SADDE: Scaled Absorbed Dose Distribution Evaluator has been modified to allow the generation of scaled absorbed dose distributions for mixtures of radionuclides and internal conversion and Auger electrons. This new code, SADDE Mod 2, is also described in this document. Instructions for installation and operation of the code and detailed descriptions of the models used in the code are provided.
\end{abstract}




\section{CONTENTS}

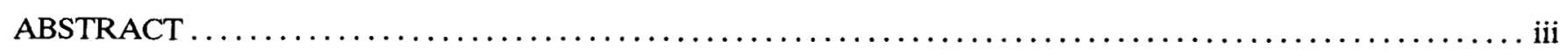

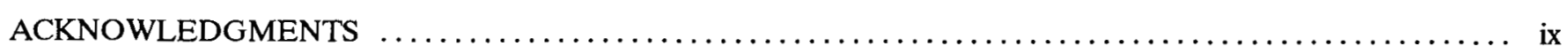

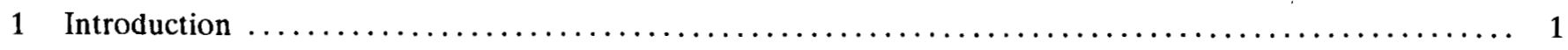

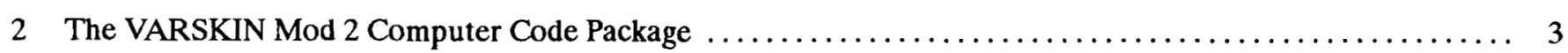

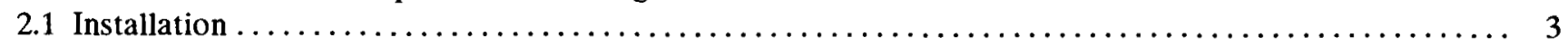

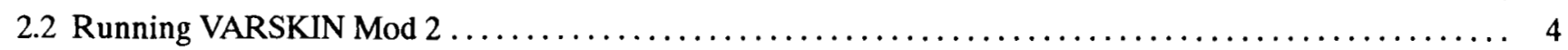

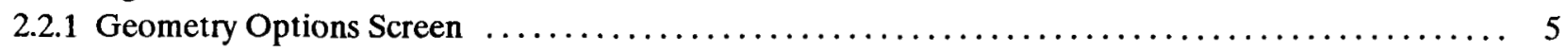

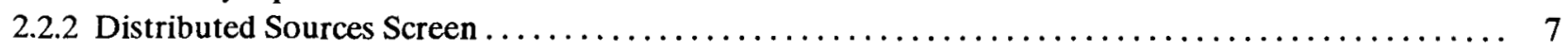

2.2.3 Radionuclide Selection and Source Strength Screen $\ldots \ldots \ldots \ldots \ldots \ldots \ldots \ldots \ldots \ldots \ldots \ldots \ldots \ldots$

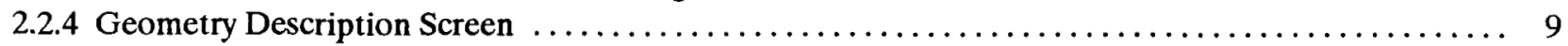

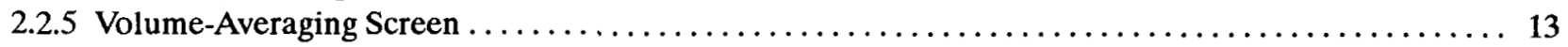

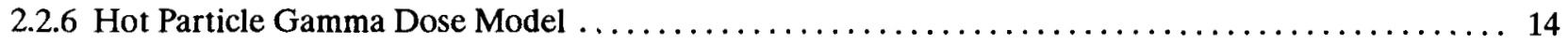

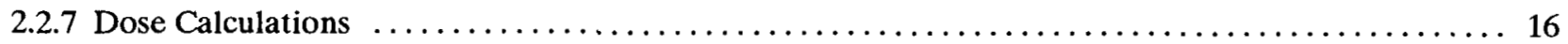

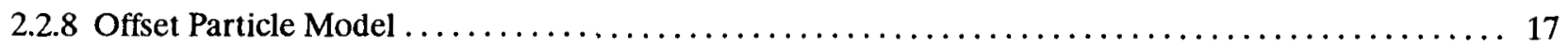

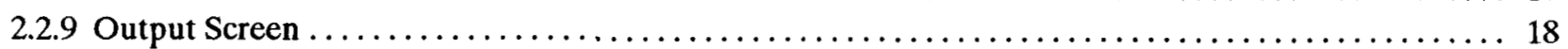

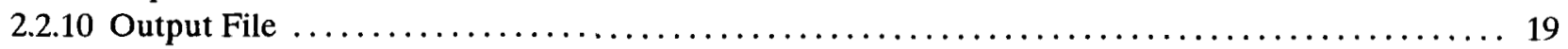

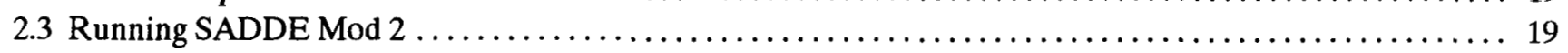

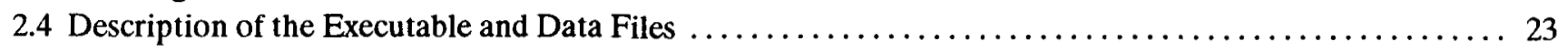

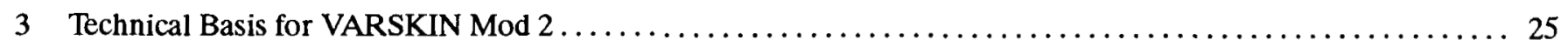

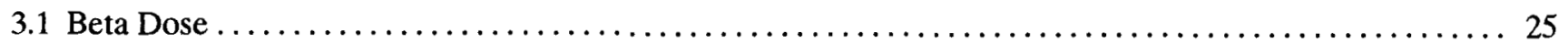

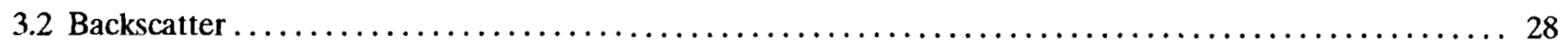

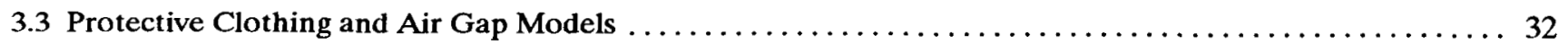

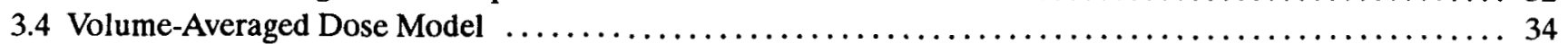

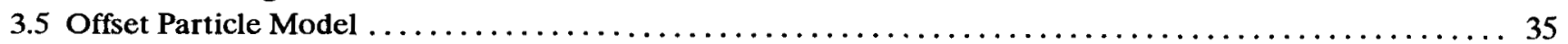

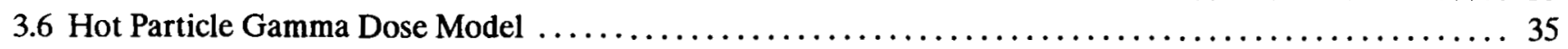

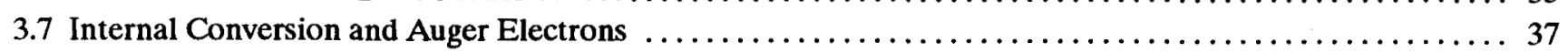

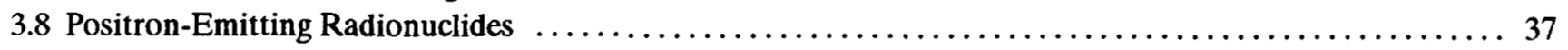

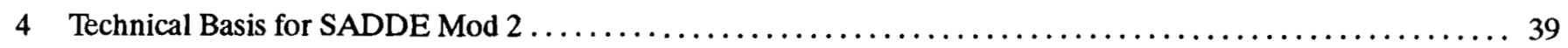

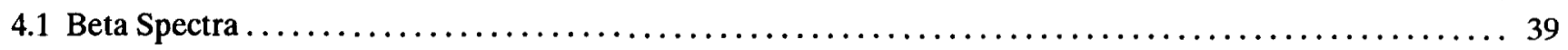

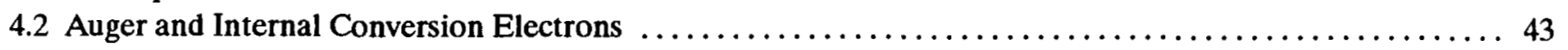

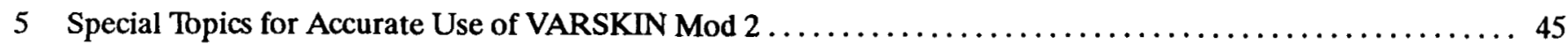

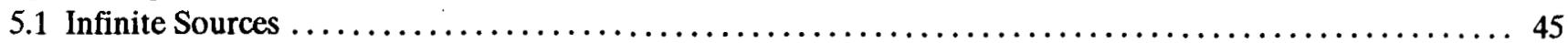

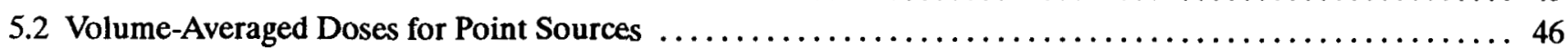

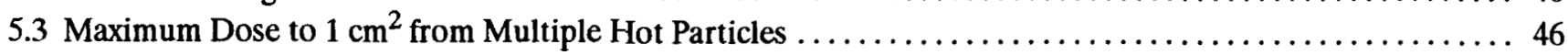

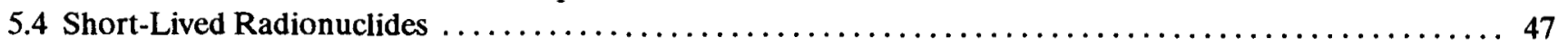




\section{Contents}

6 References

APPENDIX A - BETA-DECAY RADIONUCLIDE DATABASE

APPENDIX B - INDEPENDENT VERIFICATION OF VARSKIN MOD 2 DOSE CALCULATIONS

APPENDIX C - TEXT OF VARSKIN MOD 2 CODE

APPENDIX D - TEXT OF THE USER INTERFACE MODULES

APPENDIX E - TEXT OF SADDE MOD 2 CODE

\section{FIGURES}

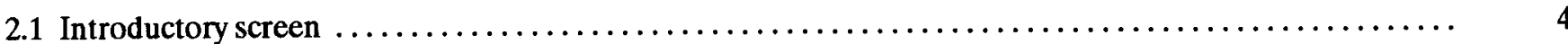

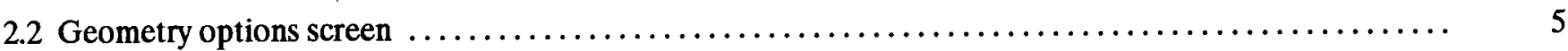

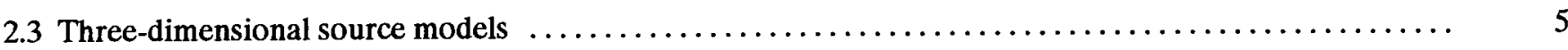

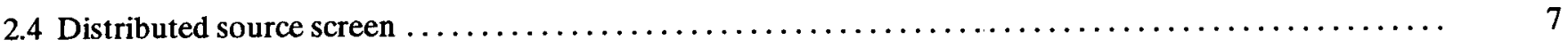

2.5 Radionuclide selection and source strength screen $\ldots \ldots \ldots \ldots \ldots \ldots \ldots \ldots \ldots \ldots \ldots \ldots \ldots \ldots$

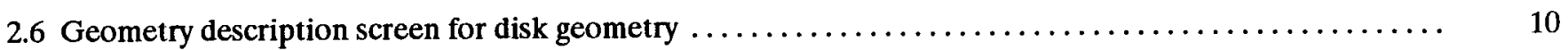

2.7 Schematic of the protective clothing and air gap models $\ldots \ldots \ldots \ldots \ldots \ldots \ldots \ldots \ldots \ldots \ldots \ldots \ldots \ldots \ldots \ldots$

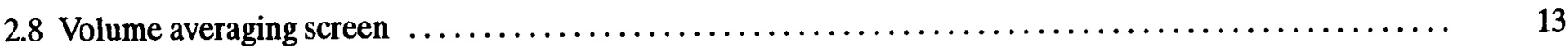

2.9 Schematic drawing of the volume averaged dose model $\ldots \ldots \ldots \ldots \ldots \ldots \ldots \ldots \ldots \ldots \ldots \ldots \ldots . . \ldots \ldots$

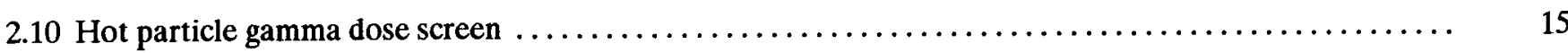

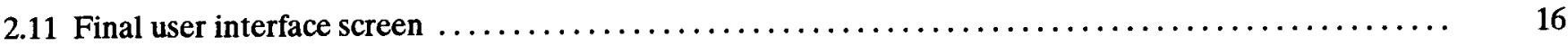

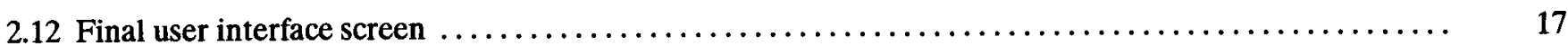

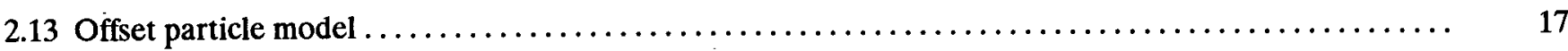

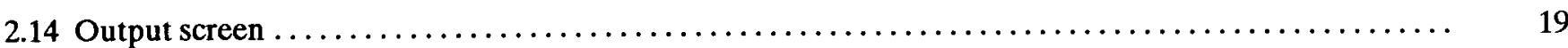


3.1 Schematic representation of a dose calculation for a three-dimensional

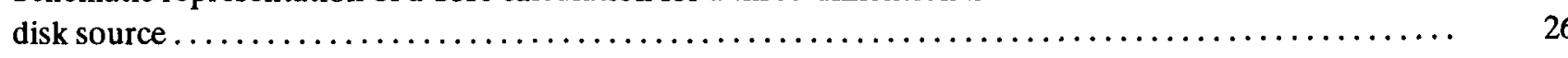

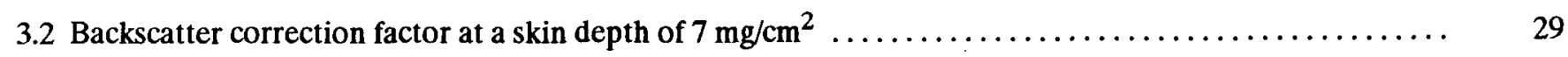

3.3 Backscatter model as a function of $\mathrm{X}_{90}$ thickness based on Cross's backscatter model .............. 30

3.4 Schematic drawing of a generic dosed calculation performed by VARSKIN Mod 2

for the three-dimensional disk geometry $\ldots \ldots \ldots \ldots \ldots \ldots \ldots \ldots \ldots \ldots \ldots \ldots \ldots \ldots \ldots \ldots \ldots \ldots \ldots \ldots$

TABLES

1 Types and abundances of a combination of radionuclides comprising a 65-day aged fuel particle based on the distribution published by Bray et al. (1987) $\ldots \ldots \ldots \ldots \ldots \ldots \ldots \ldots \ldots \ldots \ldots$

2 Typical thicknesses and densities of protective clothing covering materials $\ldots \ldots \ldots \ldots \ldots \ldots \ldots \ldots \ldots$

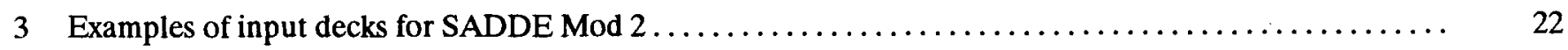

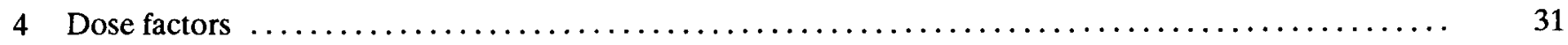




\section{ACKNOWLEDGMENTS}

The author would like to acknowledge the following people outside the Pacific Northwest Laboratory, the developer of this software, who helped test VARSKIN Mod 2:

\section{Ross Hirning, Ontario Hydro}

J. P. Burion and D. Delacroix, C. E. Saclay Bradley Parfitt, GPU Nuclear

Rick Traub, EG\&G Mound Applied Technologies Christian Wernli, Paul Scherrer Institut Douglas Stretch, Union Electric

Dale S. Cunningham, Westinghouse Hanford Corporation

Paul D. Rittman, Westinghouse Hanford Corporation

Dick Olsher, Los Alamos National Laboratory

John Lepo, Pennsylvania Power and Light
The author would also like to acknowledge the efforts of W. Dan Reece, Texas A\&M University, for the development of the user interface and the backscatter model; Gamal Akabani for compiling the input data for SADDE Mod 2; Walter E. Wilson for calculating the percentile-distance ratios and scaled absorbed dose distributions for low-energy primary electrons; and Robert D. Stewart for performing EGS4 calculations to verify VARSKIN Mod 2 results.

Finally, the author would like to thank Ila Stephens, group secretary; Marianna Cross, word processor; and Jim Weber, technical editor, for the production of this document. 


\section{FORWORD}

The original VARSKIN code has been used for several years as a tool for helping in the assessment of personnel doses from skin contamination, including so-called "hot particles." Although useful in skin contamination dose assessments, VARSKIN tended to provide overly conservative dose results for hot particles because of its inability to account for source selfshielding and source geometry effects. Also, the code was unable to accommodate those situations where sources are not directly on the skin (e.g., on clothing or hair) and are shielded by clothing or protective equipment. With beta-gamma emitting sources shielded by clothing or protective equipment, the gamma dose assumes more importance but could not be calculated with VARSKIN.

As a result of these recognized shortcomings, a new code has been developed to allow more accurate skin dose assessments with improved flexibility in a more "user-friendly" format. The new code has been extensively peer-reviewed and validated.

Although the new code was developed for use by NRC personnel for performing skin dose calculations needed in carrying out their regulatory duties, it is hoped that, like the original VARSKIN code, it will prove useful to other members of the radiation protection community.

NUREG/CR-5873 is not a substitute for NRC regulations, and compliance is not required. The approaches and/or methods described in this NUREG are provided for information only. Publication of this report does not necessarily constitute NRC approval or agreement with the information contained herein.

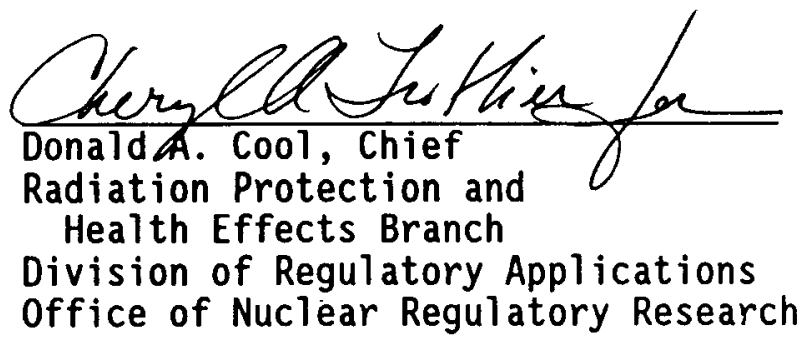




\section{Introduction}

The original VARSKIN computer code (Traub et al. 1987) was intended for calculation of skin dose from contamination directly on the skin. The contamination was assumed to be an infinitely thin layer of radioactive material distributed over an area or at a single point on the skin. Soon after the release of VARSKIN, a "new" type of skin contaminant was encountered in the industry. This contaminant consists of discrete microscopic radioactive particles, called "hot" particles. These particles differ radically from uniform skin contamination in that the particles have a thickness associated with them and many of the exposures result from particles on the outside of protective clothing. Since no other option for calculating skin dose from hot particles was readily available, skin dose calculations from hot particles were performed using VARSKIN.

Calculating skin doses from hot particles using VARSKIN has three primary drawbacks. First, VARSKIN models the contaminant as having no thickness; thus, source self-shielding is not taken into account in the calculation. For many hot particles consisting of the weak beta-emitting radionuclide ${ }^{60} \mathrm{Co}$, VARSKIN greatly overestimates calculated skin doses, including particles that are thicker than about $1 \mu \mathrm{m}$. Second, VARSKIN does not model protective clothing that is sometimes between the source and skin, again leading to an overestimate of skin dose from beta-emitting fuel particles. Finally, it has been shown that the gamma component of a mixed beta/gamma-emitting hot particle becomes increasingly important as the skin depth and, most importantly, the thickness of protective clothing increase. Since VARSKIN does not model protective clothing, the gamma dose is not generally significant and no gamma dose model was included. Thus, an upgrade of VARSKIN was needed to provide accurate skin dose calculations.

The upgraded computer code VARSKIN Mod 2 contains all of the features of the original VARSKIN with numerous significant additions. Additional features in VARSKIN Mod 2 include modeling of three-dimensional sources (thick disks, spheres, and slabs), modeling of materials placed between the source and skin (including air gaps), modeling of hot particle gamma doses in certain cases, and methods to model multiple hot particles and volume-averaged doses. VARSKIN Mod 2 also uses a correction for backscatter for one- and two-dimensional sources in certain cases. In addition, the VARSKIN Mod 2 package incorporates a user interface that greatly simplifies data entry for calculating skin dose in addition to providing on-line guidance in the form of help screens.

VARSKIN Mod 2 includes models for particle self-shielding and attenuation by protective clothing and air gaps. Also included in the upgraded code are volume averaging and offset particle models. The volume-averaging model allows the user to calculate the dose averaged over a volume defined by two different skin depths instead of the dose at one given depth. The offset particle model, which allows dose to be calculated for a particle that is not centered over the dose area of interest, is useful for calculating dose from multiple hot particles. Finally, the operation of 
Introduction

VARSKIN Mod 2 is facilitated by a user interface with on-line help and the capability to use composite source terms that allow a user to calculate the total dose from a mixture of beta-emitters in the same amount of time required for one radionuclide.

The upgraded code is based on the same Berger kernel used in VARSKIN (Berger 1971), but the integration is more complex. Instead of integrating over two source dimensions as in VARSKIN, VARSKIN Mod 2 integrates over three source dimensions. The added dimension necessarily requires significantly more execution time.

The input data file for VARSKIN Mod 2, BETADATA.DAT, has undergone significant revision. Note that previous versions of BETADATA.DAT (supplied with VARSKIN) will not allow VARSKIN Mod 2 to calculate doses. The old file should be removed from the system or stored separately from VARSKIN Mod 2 files. The new BETADATA.DAT file has been modified to reflect more recent physical data for the average energy, maximum energy, and yield based on data generated by the computer code RADLST (Burrows 1988). Additional information on RADLST is given in Appendix B. Further modifications include adding data for gamma dose calculations and including contributions from internal conversion and Auger electrons.

Also included with the VARSKIN Mod 2 package is an upgraded (Mod 2) version of SADDE: Scaled Absorbed Dose Distribution Evaluator (Reece et al. 1989). The original version of SADDE calculated the scaled absorbed dose distribution functions that were input to VARSKIN. The upgraded version, SADDE Mod 2, contains all of the capabilities of the original version, with the additional feature of allowing the user to develop scaled absorbed dose distributions for mixtures of up to 30 radionuclides. To facilitate the use of SADDE Mod 2, input to the code for virtually every beta-emitting radionuclide is included in Appendix A. SADDE Mod 2 uses scaled absorbed dose distributions for monoenergetic electrons that have been extended down to energies of $50 \mathrm{eV}$. Finally, SADDE Mod 2 can be used to include doses from internal conversion and Auger electrons when they accompany beta emission.

Section 2.0 of this report describes the contents of the VARSKIN Mod 2 and SADDE Mod 2 code packages, including instructions for installing each package on a DOS-system personal computer and for running the package. Section 3.0 discusses the technical basis for VARSKIN Mod 2, the skin dose calculation portion of the package, including the calculational methodology. Section 4.0 presents the models and required input to SADDE Mod 2, the upgraded version of SADDE. Section 5.0 alerts users to possible misuse of the VARSKIN Mod 2 package and explains the correct method for modeling semi-infinite sources. 


\section{The VARSKIN Mod 2 Computer Code Package}

\subsection{Installation}

This section describes the procedures for installing the VARSKIN Mod 2 and SADDE Mod 2 computer codes on a personal computer. It also describes the separate codes in the code package, some of which are executed via a single batch file and some of which must be executed separately. VARSKIN Mod 2 and SADDE Mod 2 may be obtained from Energy Science and Technology Software Center (ESTSC), P.O. Box 1020, Oak Ridge, TN 37831-1020, or the Radiation Shielding Information Center (RSIC), Oak Ridge National Laboratory, P.O. Box 2008, Oak Ridge, TN 37831-6367. The telephone number for ESTSC is (615) 576-2606 and for RSIC it is (615) 574-6176. A nominal fee may be required.

Both VARSKIN Mod 2 and SADDE Mod 2 are designed for use on personal computers in a DOS environment. The codes are supplied on 3.5-in. or 5.25-in. diskettes. The source codes for the programs are also provided.

The most efficient operation of VARSKIN Mod 2 and SADDE Mod 2 is realized by installing them on a hard disk drive in a computer with a math coprocessor. The user should create a subdirectory dedicated to the VARSKIN Mod 2 code package, which includes both VARSKIN Mod 2 and SADDE Mod 2. To create a subdirectory use the following command at the prompt:

\section{MKDIR VARSKIN}

Next, switch to the VARSKIN subdirectory using the command

\section{VARSKIN}

To install the code package, put the diskette into the appropriate drive (usually the 'A' drive). If the computer on which the code is to be installed has a math coprocessor, use the command

a:CD math

to change to the subdirectory on the distribution diskette that contains the codes for computers with a math coprocessor. Then use the command

copy $a^{*} .^{*}$

If the personal computer is not supplied with a math coprocessor, use the command

a: cd emul

to change to the subdirectory on the distribution diskette that contains VARSKIN Mod 2 for computers without a math coprocessor. Then, type the command

copy $\mathrm{a}^{* *} \cdot *$ 
If the personal computer does not have a hard drive, VARSKIN Mod 2 can be run from a floppy disk, but the execution time will be significantly increased. A backup copy of the distribution diskette should be made before attempting to use either VARSKIN Mod 2 or SADDE Mod 2. Follow the above instructions for the hard disk installation but substitute the drive letter of the target diskette for the hard drive designation.

A third subdirectory, entitled SOURCE, contains all of the FORTRAN and C source codes for the executable files. These files are not required to execute either VARSKIN Mod 2 or SADDE Mod 2, and are included to allow a user to make changes or review the programming of each of the two codes. The FORTRAN codes were compiled using Microsoft optimizing FORTRAN Compiler Version 5.1. The $\mathrm{C}$ modules were compiled using Microsoft $\mathrm{C}$ compiler Version 6.0.

To begin VARSKIN Mod 2, enter the following command at the prompt:

\section{VARMOD2}

A short title screen, shown in Figure 2.1, will appear. Hitting any key at this point will initiate the user interface module, which allows data entry in VARSKIN Mod 2. The statement "Reading Data - Please Wait" will appear for up to 20 seconds (depending on the speed of the computer) after pressing any key while the BETADATA.DAT file is read by the user interface.

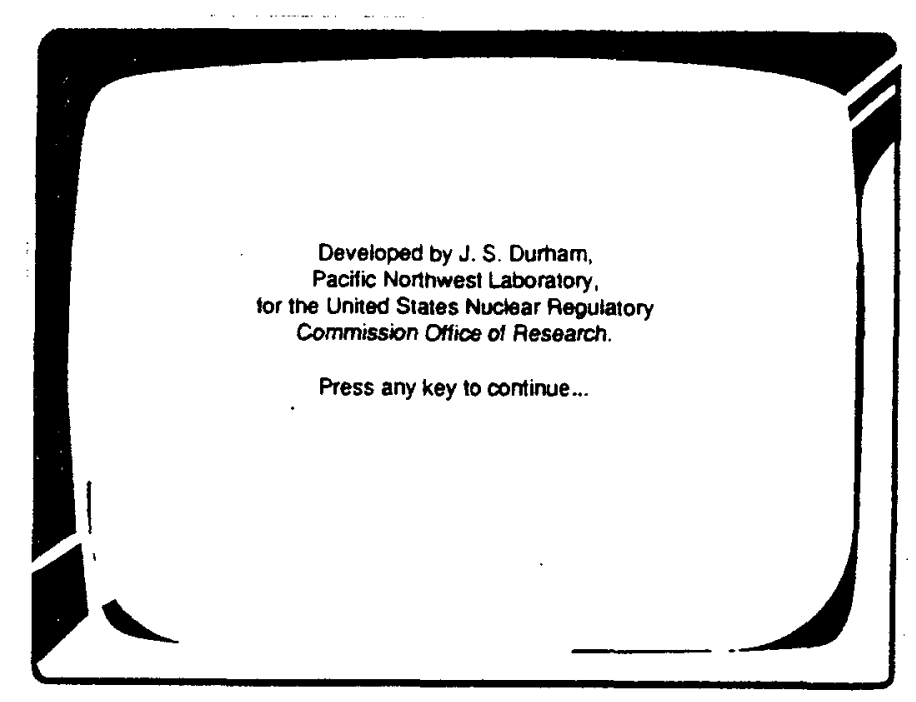

Figure 2.1 Introductory screen 


\subsubsection{Geometry Options Screen}

The geometry options screen, shown in Figure 2.2, lists the five available geometry options in VARSKIN Mod 2: Point, 2-D disk (identical to the previous version of VARSKIN), 3-D disk, Sphere, and 3-D Slab. Representative drawings of the three models are provided in Figure 2.3. A brief description of each model follows.

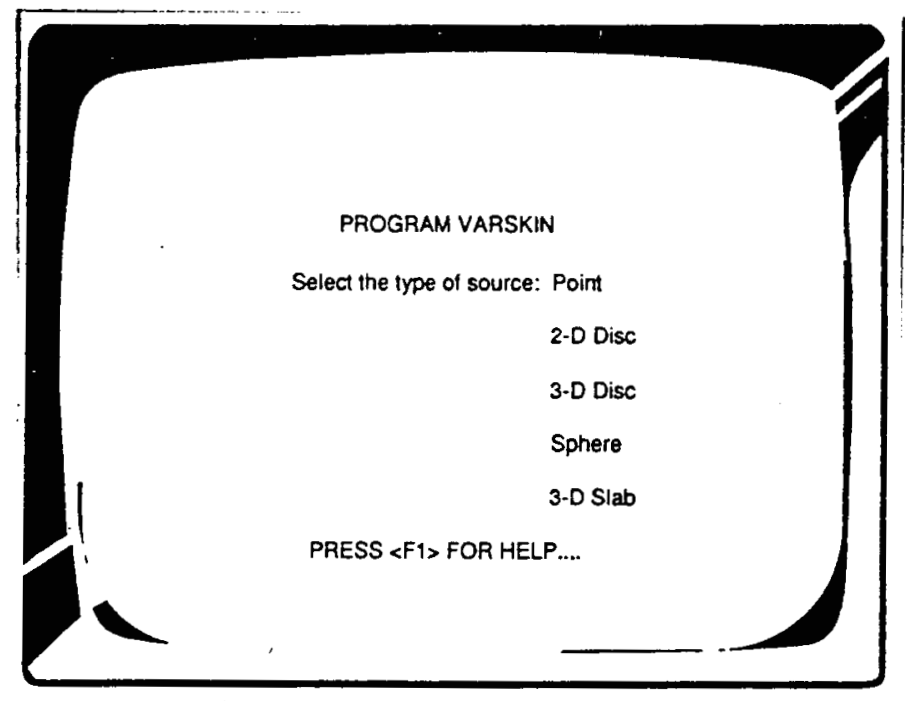

Figure 2.2 Geometry options screen

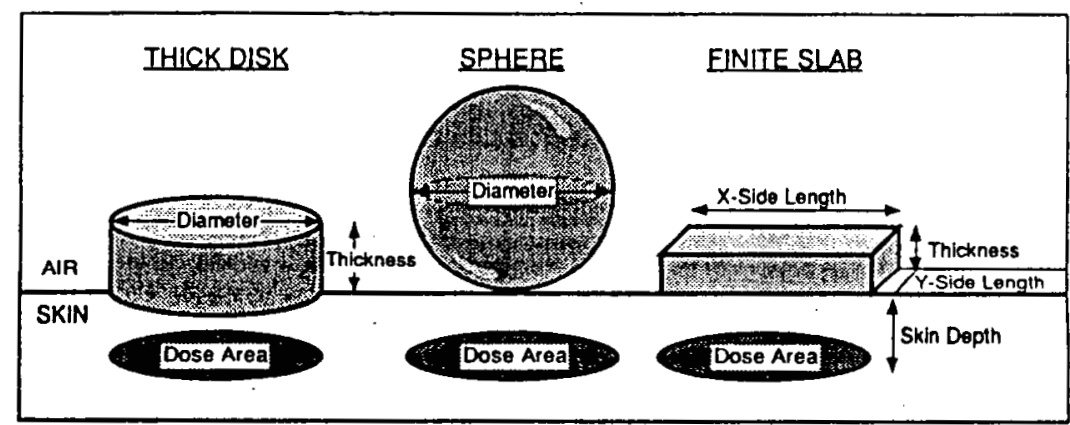

Figure 2.3 Three-dimensional source models

The spherical geometry model is perhaps the simplest to use because it requires knowledge of only one source dimension: the sphere diameter. The sphere model assumes that the source is surrounded by air and touches the skin or protective clothing only at the bottom-most point on the sphere surface. The air surrounding the sphere does not shield the source particles as efficiently as in the disk and slab models, and the average path length within a spherical source is at a minimum; therefore, doses calculated using the spherical model will be consistently higher than those calculated using the other models. Because hot particles seldom resemble spheres, this model is of more limited utility than the other two models. 
The three-dimensional disk model requires knowledge of two dimensions: the disk diameter and the disk thickness. The disk model assumes that the source is surrounded by air but that the entire bottom of the disk is in contact with skin or protective clothing. This model requires the same amount of time to execute as the spherical model but offers a more practical application. Of the two disk source dimensions, the calculated dose is much more sensitive to changes in the disk thickness than in the disk diameter. For low-energy beta emitters, the difference in the calculated dose averaged over $1 \mathrm{~cm}^{2}$ at a skin depth of $70 \mu \mathrm{m}$ for $2-\mathrm{mm}$ - and 10 -mm-diameter sources is only $11 \%$, while for high-energy sources this difference is $16 \%$, all other source parameters being equal.

The three-dimensional slab geometry model requires knowledge of three physical dimensions: the $x$-side length, the $y$-side length, and the thickness. The slab model requires 2 to 5 times more execution time than the three-dimensional disk and sphere models, and the accuracy of the calculation is significantly lower. The 3-D slab geometry model should only be used when skin dose from multiple, discrete particles is needed.

The following general rules should govern the choice of geometry package, progressing from most conservative to least conservative:

- If nothing is known about the particle size and shape, use the point source option (option 1).

- If the diameter is known but the thickness cannot be estimated, or if a distributed source is being modeled (i.e., with a known source strength per unit area), use the 2-D disk option (option 2).

- If the thickness and the diameter can be estimated but the shape is not known, use the 3-D disk option (option 3), because this geometry requires only two dimensions (thickness and radius) to describe the particle.

- If the particle is known to be spherical and is not imbedded in another material, use only the spherical geometry option (option 4).

- If the particle is known to be rectangular, use the 3-D disk geometry (option 5). The thickness of the particle should be maintained, and the area of the contact surface should be equivalent. This option requires less time to run than the 3-D slab model and is more accurate.

Note the statement in Figure 2.2, "PRESS <F1 > FOR HELP...." The user interface to VARSKIN Mod 2 contains help screens for most of the input parameters. The help screen will, in general, repeat what is stated in this document for the parameter of interest. 


\subsubsection{Distributed} Sources Screen
Once the geometry has been selected, the distributed source screen (Figure 2.4) appears. A distributed source is characterized in units of activity per unit area or volume. The source can also be characterized in terms of total activity. If the total activity of the source is known, answer this question by typing " $\mathrm{N}$ ". However, if the source is known in terms of $\mu \mathrm{Ci} / \mathrm{cm}^{2}$ or $\mu \mathrm{Ci} / \mathrm{cm}^{3}$, answer this question by typing "Y". The distributed source feature is useful for large-area contaminations.

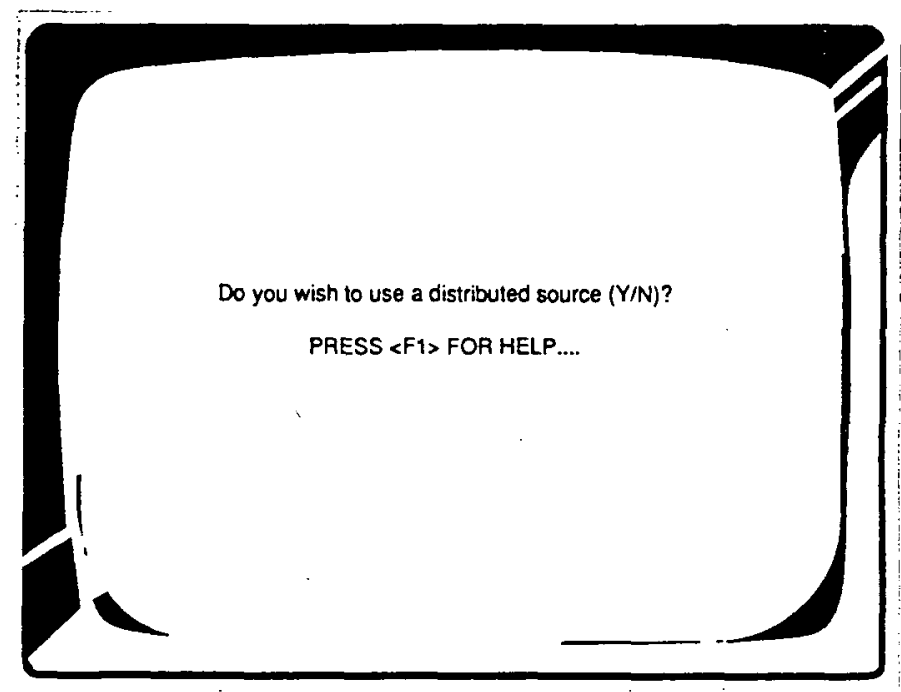

Figure 2.4 Distributed source screen

\subsubsection{Radionuclide}

Selection and Source Strength Screen
Next, the list of available radionuclides will be displayed on the screen as shown in Figure 2.5. The arrow keys are used to move around the screen and highlight the desired radionuclide. When the desired radionuclide is highlighted, press $<$ Return $>$. At this point, a window will open that requests the source strength either in $\mu \mathrm{Ci}, \mu \mathrm{Ci} / \mathrm{cm}^{2}$, or $\mu \mathrm{Ci} / \mathrm{cm}^{3}$, depending on the response to the question on the distributed source screen. Enter the source strength in the appropriate units at this point. When the source strength is entered, the list of available radionuclides appears again and another radionuclide can be selected. VARSKIN Mod 2 can be used to calculate the dose from up to five radionuclides in a single execution. 


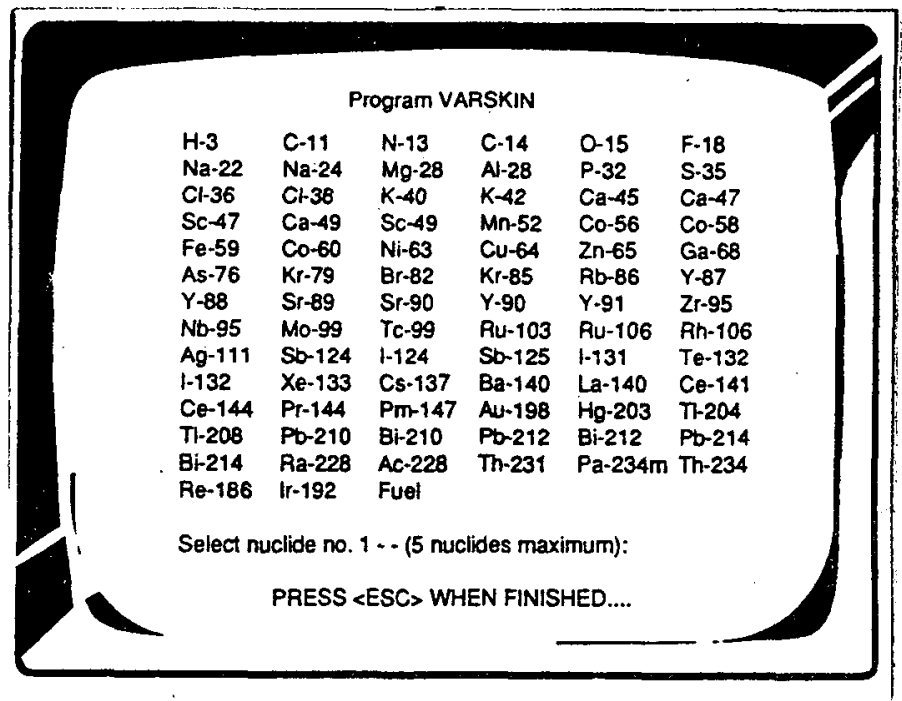

Figure 2.5 Radionuclide selection and source strength screen

Note that one of the "radionuclides" in the list is entitled "Fuel." This entry serves as an example of the type of composite source that can be prepared using SADDE Mod 2. This source is a composite of 16 weighted radionuclides that comprise a 65-day-old fuel particle (Bray et al. 1987). The fuel particle includes each of the radionuclides listed in Table 1, yet requires the same execution time as for a single radionuclide. Choosing "Fuel" as the radionuclide will provide an estimate of skin dose from an aged fuel particle. Only the beta dose is calculated when "Fuel" is chosen as the source since the list of radionuclides in Table 2.1 does not include gamma emitters. 
Table 1 Types and abundances of a combination of radionuclides comprising a 65-day aged fuel particle based on the distribution published by Bray et al. (1987)

\begin{tabular}{lcc}
\hline Radionuclide & Eo $(\mathrm{MeV})$ & Fraction of Total Activity \\
\hline${ }^{89} \mathrm{Sr}$ & 1.491 & 0.0905 \\
${ }^{90} \mathrm{Sr}$ & 0.546 & 0.0085 \\
${ }^{90} \mathrm{Y}$ & 2.284 & 0.0085 \\
${ }^{91} \mathrm{Y}$ & 1.543 & 0.1278 \\
${ }^{95} \mathrm{Zr}$ & 0.123 & 0.1097 \\
${ }^{95} \mathrm{Nb}$ & 1.160 & 0.1640 \\
${ }^{103} \mathrm{Ru}$ & 0.723 & 0.0650 \\
${ }^{106} \mathrm{Rh}$ & 3.541 & 0.0458 \\
${ }^{106} \mathrm{Ru}$ & 0.039 & 0.0458 \\
${ }^{140} \mathrm{Ba}$ & 1.005 & 0.0112 \\
${ }^{140} \mathrm{La}$ & 2.164 & 0.0192 \\
${ }^{141} \mathrm{Ce}$ & 0.580 & 0.0639 \\
${ }^{143} \mathrm{Pr}$ & 0.935 & 0.0075 \\
${ }^{144} \mathrm{Ce}$ & 0.318 & 0.1331 \\
${ }^{144} \mathrm{Pr}$ & 2.996 & 0.1331 \\
${ }^{147} \mathrm{Pm}$ & 0.225 & 0.0288 \\
${ }^{\mathrm{TOTAL}}$ & & 1.000 \\
\hline
\end{tabular}

\subsubsection{Geometry} Description Screen
The geometry description screen, shown for a three-dimensional disk source in Figure 2.6, contains a description of the geometry that is to be modeled. The input parameters include the dimensions of the source, the irradiation time in minutes, the density of the source if necessary, the relative error (see discussion below), the thickness of any air gap, and the thickness and density of any protective clothing. Source dimensions are entered in micrometers; all densities are in grams per cubic centimeter; and the air gap and protective clothing thicknesses are in millimeters. The user can enter the values simply by using the arrow keys to highlight the desired entry. If a value for a given variable is to be changed, the original value for that parameter must be erased by striking < Delete $>$ once before entering the new variable. If the delete key is not used, then the new value will be appended to the existing value, possibly resulting in erroneous data entry. 


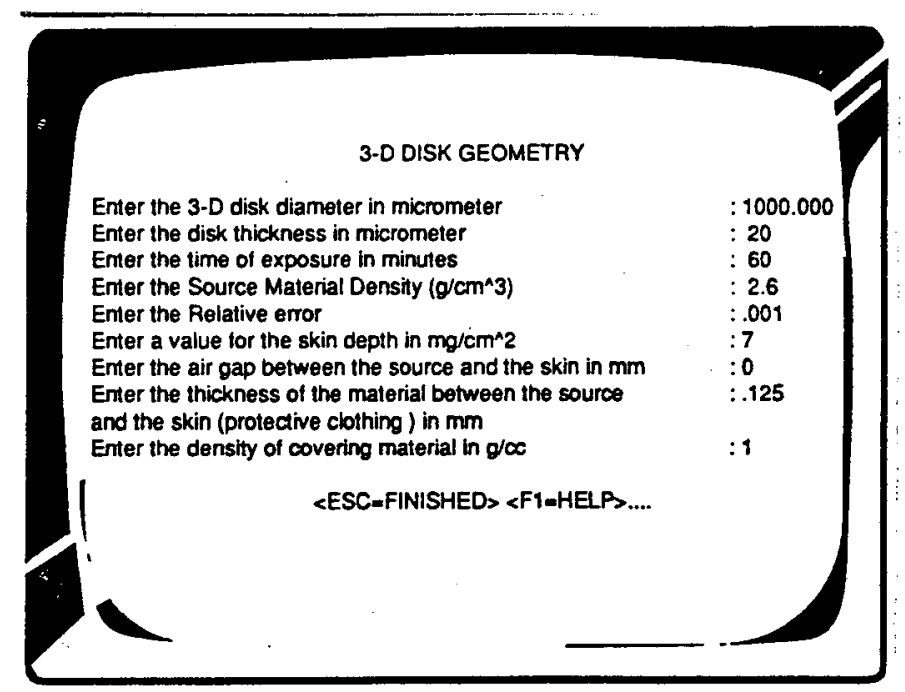

Figure 2.6 Geometry description screen for disk geometry

The two-dimensional disk geometry package has a unique feature. The user has the option of entering either the source radius or the source area in $\mathrm{cm}^{2}$. This feature simplifies data entry for two-dimensional sources where the area and the total microcurie inventory are known.

A sensitivity analysis of many of the geometry input parameters for VARSKIN Mod 2 has been published (Durham et al. 1991). The results of the analysis show that the most sensitive source dimension for calculating dose is the source thickness, and that the dose will vary greatly for small variations in the value of this parameter. Similarly, the source density is an equally sensitive parameter. It is essential that these parameters be known accurately or that a conservative estimate of their values be made. The following guidelines will help in choosing appropriate values for source dimensions and density:

1. Diameter (2-D and 3-D disks); X-side and Y-side lengths (3-D Slab)

The dose calculation for most radionuclides is relatively insensitive with respect to these dimensions for sizes below 1-2 mm. A conservative dose estimate can be made by choosing a larger rather than a smaller value for this dimension.

\section{Thickness (3-D disk, 3-D Slab, and Sphere Diameter)}

The dose calculation for all radionuclides is very sensitive to this dimension, especially for low-energy beta-emitting radionuclides. A conservative dose estimate can be made by minimizing the value of this dimension. 


\section{Source Density (3-D geometries)}

Choose a source density that is consistent with the radionuclide within the source. For hot particle contaminations, a typical density for stellite $\left({ }^{60} \mathrm{Co}\right)$ is $8.3 \mathrm{~g} / \mathrm{cm}^{3}$ and for fuel is $14 \mathrm{~g} / \mathrm{cm}^{3}$.

Additional guidance and several examples of the sensitivity of these calculations can be found in the literature (Durham et al. 1991). The above guidance is also available in the form of a help screen, which may be viewed by highlighting the appropriate variable and pressing $<\mathrm{F} 1>$.

The next variable to input is the relative error, which must be between zero and one. This value controls the convergence of the numerical integration. (Additional information on the integration is provided in Section 4.) The recommended value of the relative error is 0.001 , which corresponds to a convergence level of $0.1 \%$. A larger value may result in decreased execution time, but with reduced precision. Similarly, a smaller value may provide greater precision but can require a larger execution time.

The next input is the skin depth in $\mathrm{mg} / \mathrm{cm}^{2}$. The default value is $7 \mathrm{mg} / \mathrm{cm}^{2}$, the depth recommended by the NRC for skin dose calculations.

The presence of protective clothing and air gaps can be chosen separately or together. A representative drawing of the two models is shown in Figure 2.7, which depicts the three-dimensional disk geometry, although the two models are available for any of the source geometries. The required input for the protective clothing model is the thickness (in $\mathrm{mm}$ ) of the clothing and its corresponding density in grams per cubic centimeter. Note that both parameters are needed to account for the $1 / \mathrm{r}^{2}$ dependence of the Berger point kernel (geometric attenuation) and for the energy loss due to attenuation in the material (material attenuation). For the air gap model, only the thickness of the air gap is required for input. VARSKIN Mod 2 uses a default value of $0.001293 \mathrm{~g} / \mathrm{cm}^{3}$ for air density.

Air gap and protective clothing physical characteristics can significantly affect the calculated skin dose. While the air gap has little effect on material attenuation, its effect on geometric attenuation can be dramatic. Protective clothing affects both the geometric and material attenuation. Some suggested thickness and density values are given in Table 2. VARSKIN Mod 2 allows only one cover material and one air gap to be modeled. However, composite cover materials can be modeled using the following procedure. 


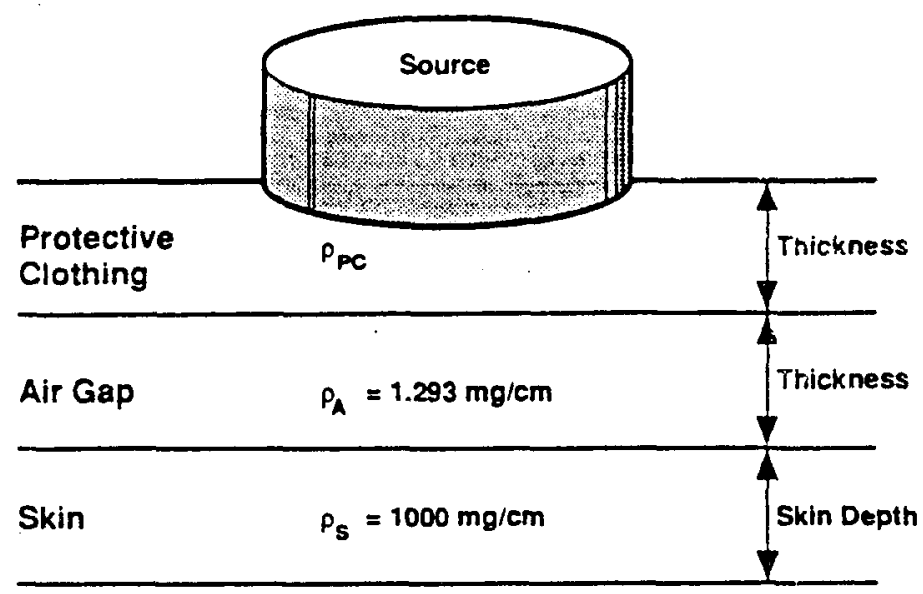

Figure 2.7 Schematic of the protective clothing and air gap models

Table 2 Typical thicknesses and densities of protective clothing covering materials

\begin{tabular}{lcc}
\hline \multicolumn{1}{c}{ Material } & $\begin{array}{c}\text { Thickness } \\
(\mathrm{mm})\end{array}$ & $\begin{array}{c}\text { Density } \\
\left(\mathrm{g} / \mathrm{cm}^{3}\right)\end{array}$ \\
\hline Lab Coat (plastic) & 0.1 & 0.036 \\
Cotton Glove Liner & 0.3 & 0.3 \\
Surgeon's Glove & 0.5 & 0.9 \\
Outer Glove (thick) & 0.45 & 1.1 \\
Ribbed Outer Glove & 0.55 & 0.9 \\
Plastic Bootie & 0.2 & 0.6 \\
Rubber Shoe Cover & 1.2 & 1.0 \\
Coveralls & 0.7 & 0.4 \\
\hline
\end{tabular}

Both the density $\left(\mathrm{g} / \mathrm{cm}^{3}\right)$ and thickness ( $\mathrm{mm}$ ) of each covering material must be known. To obtain the thickness ( $\mathrm{mm}$ ) of the composite material, sum the thicknesses $(\mathrm{mm})$ of the covering materials. To obtain the density of the composite material, multiply the density of each material by its thickness $(\mathrm{cm})$ to obtain the density thickness $\left(\mathrm{g} / \mathrm{cm}^{2}\right)$ of each covering material; then, sum the density thicknesses of the covering materials and divide this number by the total thickness of the composite material. The result is the density of the composite material in grams per cubic centimeter. Be sure to enter the thickness of the composite material in $\mathrm{mm}$.

The thickness of an air gap will greatly influence the calculated dose rate. A conservative estimate of the dose will be obtained if the air gap is ignored or 
minimized. When the exposure geometry has been entered, the user is instructed to press $<$ Escape $>$ to accept the entered values.

\subsubsection{Volume- Averaging Screen}

The next screen, shown in Figure 2.8, will ask if the user wishes to perform volume averaging. The volume-averaging model allows the user to calculate the dose averaged between any two depths in the skin. Initially, the code calculates the dose at 20 depths between a skin depth of zero and the maximum penetration depth of the beta particles, as shown in Figure 2.9. Thus, the volume-averaging model requires up to 20 times the execution time of that for a single depth. Once the code has completed the initial calculations, the user is prompted to enter the skin depths corresponding to the top and bottom of the volume, and the volume-averaged dose is calculated almost instantaneously. The user can then enter two new skin depths or press $<$ Enter $>$ to complete the dose calculation.

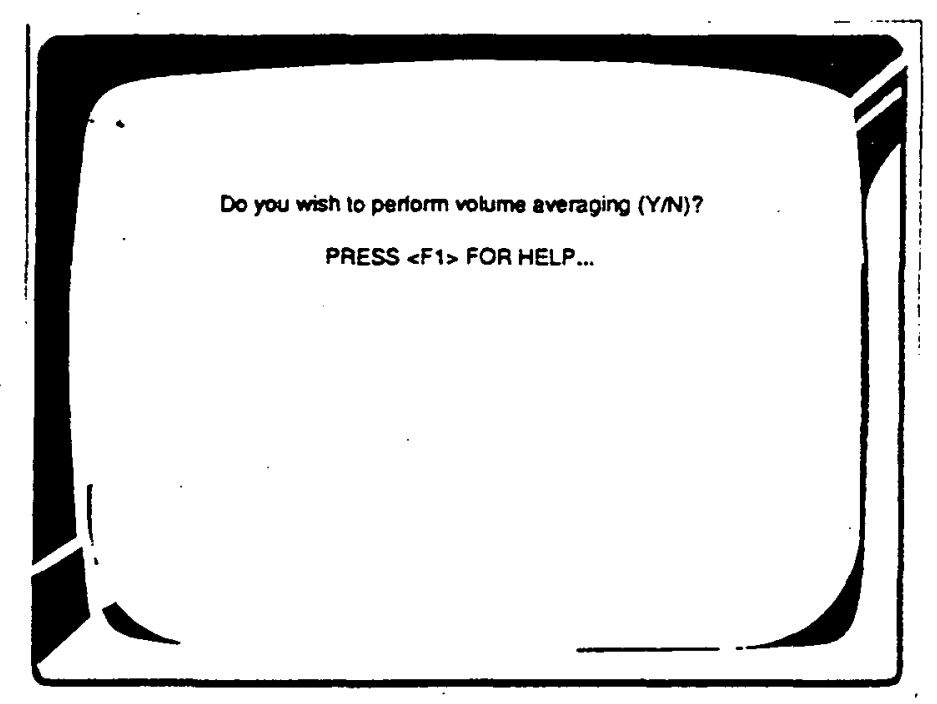

Figure 2.8 Volume averaging screen 


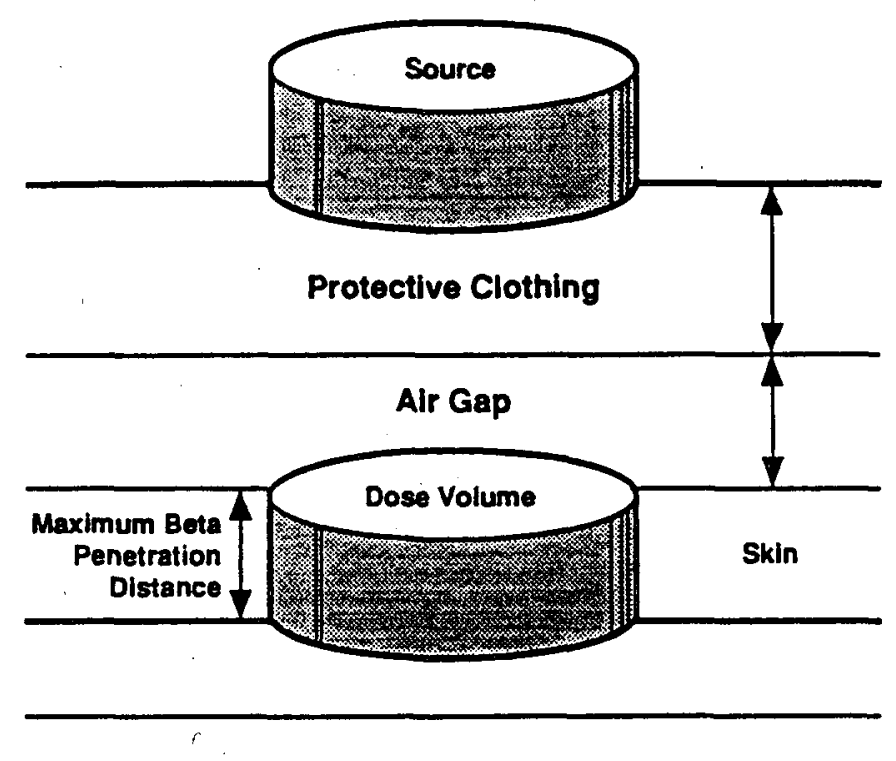

Figure 2.9 Schematic drawing of the volume averaged dose model

\subsubsection{Hot Particle Gamma Dose Model}

VARSKIN Mod 2 includes a "hot particle gamma dose" calculation routine that assumes that the source is small enough to be considered a point source and that any covering material is similar to tissue or water. The gamma dose model is based on a model published by Lantz and Lambert (1990) and is described in more detail in Section 3.6. The code will perform the gamma calculation only if the following four criteria are satisfied:

1. The radionuclide has a gamma component.

2. The diameter of the disk or sphere source, or any dimension of the slab source, is smaller than $1 \mathrm{~mm}(1000 \mu \mathrm{m})$.

3. Any air gap is smaller than $5 \mathrm{~cm}$.

4. The dose averaging area is $1 \mathrm{~cm}^{2}$.

Hot particle gamma doses are calculated as if the source were a point source, and the model includes effects due to the incomplete establishment of electronic equilibrium. Electronic equilibrium is established at different depths, depending on the average energy of the gamma photon (or photons) emitted by the particle. At shallow depths (before electronic equilibrium is established), the gamma dose is lower than the dose would be if electronic equilibrium had been fully established. The gamma dose calculation executes in less than 2 seconds. A screen, shown in Figure 2.10, asks the user if the gamma dose is to be calculated for each of the radionuclides that have a gamma component. 


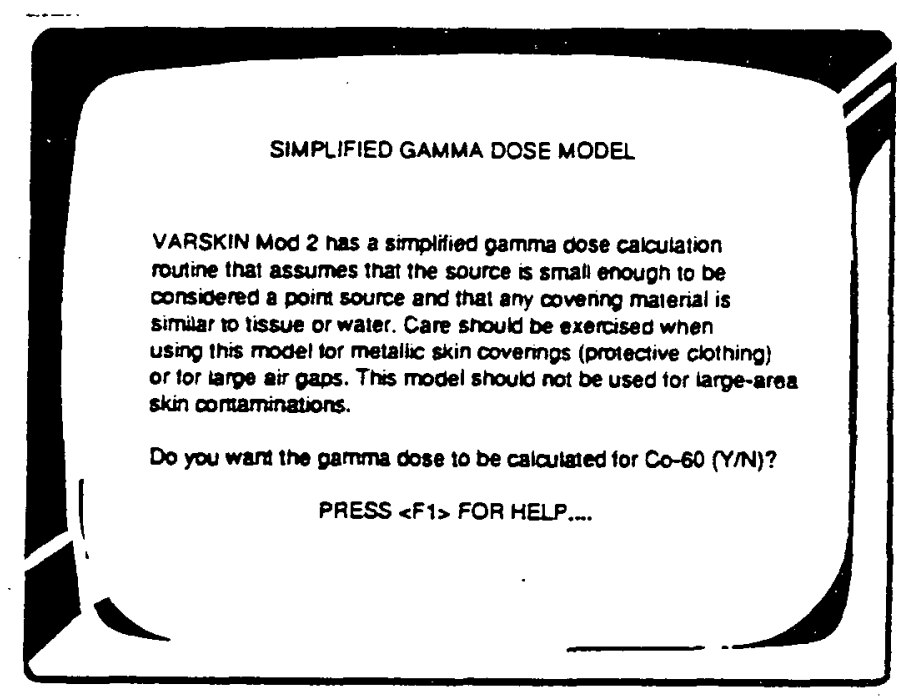

Figure 2.10 Hot particle gamma dose screen

In general, the calculated gamma skin dose will be negligible compared to the beta dose, usually by three orders of magnitude. A significant exception to this concerns ${ }^{60} \mathrm{Co}$. The relatively low energy beta particles from this radionuclide can be easily shielded by protective clothing, with little attenuation of the gamma dose. For this case, the gamma dose will account for $100 \%$ of the calculated skin dose.

Measurements verifying this phenomenon have been published by Durham and Lantz (1991).

VARSKIN Mod 2 is primarily a beta dose calculation tool. The hot particle gamma dose model is intended for use in estimating the gamma dose, averaged over $1 \mathrm{~cm}^{2}$ of skin, from a hot particle. It is not intended for use with distributed contamination. Calculations of gamma dose for distributed sources can be performed using a code designed to calculate gamma dose. An example of such a gamma dose code is ISOSHLD, available from the Radiation Shielding Information Center, Oak Ridge, TN. (ISOSHLD is only an example; no recommendation for the use of ISOSHLD is implied.)

The instruction concerning calculation of the gamma dose completes the user interface portion of VARSKIN Mod 2. The input data are written to the ASCII file OUTPUT.DAT, as shown in Figure 2.11. If the user wishes to make any changes to the input data, a provision is made for this option before beginning the dose calculation portion of VARSKIN Mod 2. However, the user must re-enter all of the source radionuclides if changes to the input data are required. 


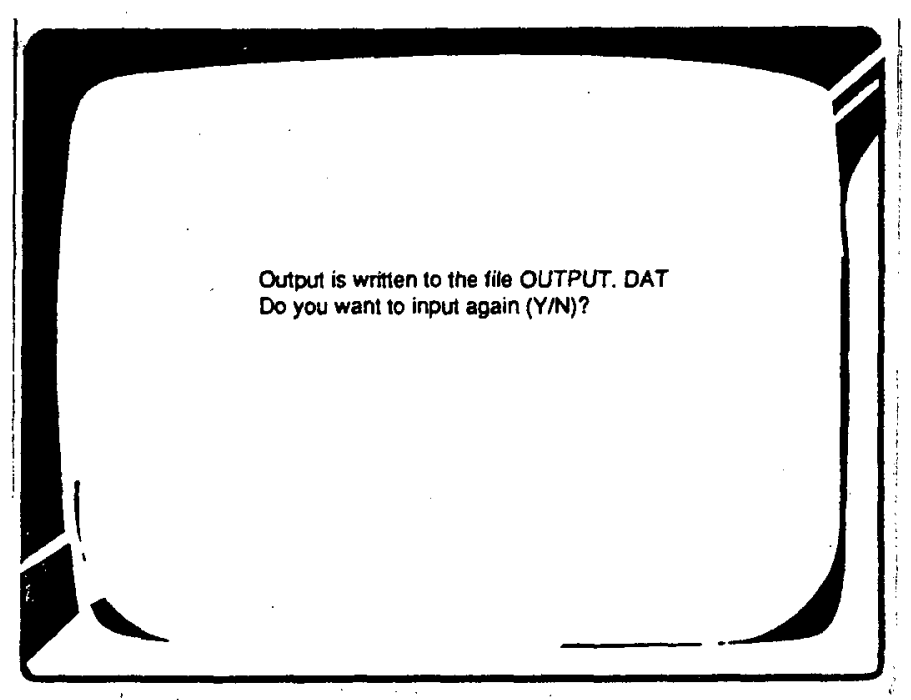

Figure 2.11 Final user interface screen

\subsubsection{Dose Calculations}




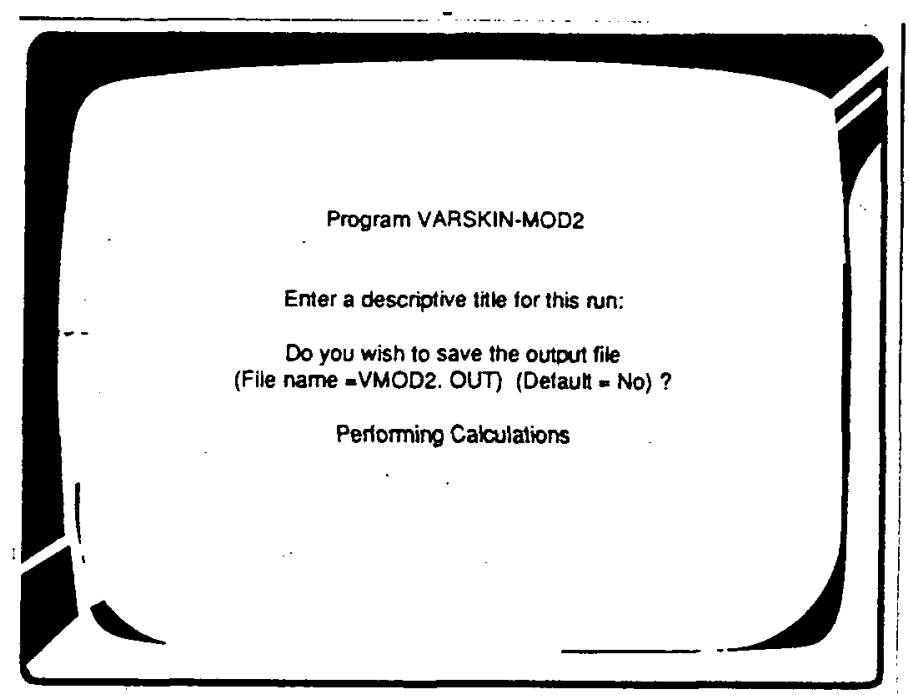

Figure 2.12 Final user interface screen

\subsubsection{Offset Particle Model}

Another option is available in VARSKIN Mod 2 for the three-dimensional slab geometry. The offset particle model allows skin dose to be calculated for a particle that is not centered over the dose area of interest and is useful for calculating dose from multiple hot particles. This model is available only for the three-dimensional slab geometry, and the offset particle must have equal $x$ - and $y$-side lengths. The offset model is pictured in Figure 2.13. As seen in the figure, the offset is the horizontal distance between the center of the offset particle and the center of the dose-averaging area. The value of the offset is the only additional input value that is required for the model, and the calculation is completed in roughly the same amount of time as a centered particle. The three-dimensional slab geometry should only be used if use of the offset particle model is desired.

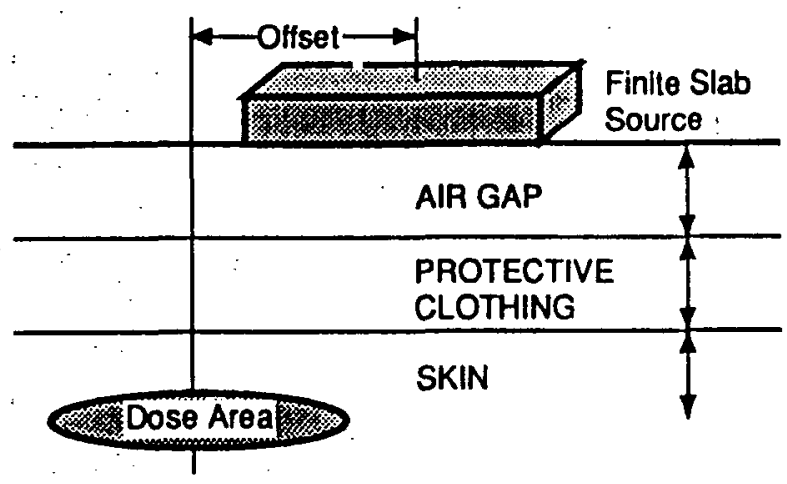

Figure 2.13 Offset particle model 


\subsubsection{Output Screen}

NUREG/CR-5873
The offset particle model can be used to calculate the dose from multiple hot particles. One of the particles is chosen to be the centered particle, and the dose to the dose-averaging area is calculated. The offset for each of the remaining particles is then determined, and the dose to the dose-averaging area is calculated for each particle. The user can then add the contributions from each particle to obtain the total skin dose to the dose-averaging area.

Next, VARSKIN Mod 2 performs the beta dose calculations. Following completion of the calculations, the user is asked if the output data file is to be saved. The name of the output file is automatically chosen by the code. If the output file is not to be saved, the user must obtain the results directly from the screen. The output file name is chosen by VARSKIN Mod 2; the user may change the output file name manually at the completion of the code. VARSKIN Mod 2 can name up to 27 different output files; if a user attempts to save more than 27 output files, VARSKIN Mod 2 will issue an error message and the user must delete or rename some of the output files.

When the dose calculations are completed, the results are displayed on the screen, as shown in Figure 2.14 for the input data in Figure 2.6. The output screen includes the input variables chosen and the results of the calculations. The first result displayed tells the user whether the irradiated area is larger or smaller than the area over which the calculation is averaged and shows the beta dose for the area of irradiation. If the irradiated area is smaller than the averaging area, the radius of the irradiated area and the dose averaged over that area is provided to the user.

The next two results present the beta dose and beta dose rate averaged over the dose area. Note that the dose area defaults to $1 \mathrm{~cm}^{2}$ for the first calculation. The last two results that are printed to the screen are the gamma dose and dose rate averaged over $1 \mathrm{~cm}^{2}$. If a given radionuclide has no gamma component, the gamma dose is omitted. If a given radionuclide has a gamma component, the total dose (gamma plus beta) is displayed.

One final option exists for VARSKIN Mod 2. If the user wishes to repeat the calculations for the same radionuclide(s) and geometry but for a different skin area (the initial value of the skin area is $1 \mathrm{~cm}^{2}$ ), the new value is entered at the prompt, "Enter new area over which to average dose." If $<$ Return $>$ or a non-positive number is entered, the code will terminate, the output file will be saved (if desired), the red border around the screen will be erased (math coprocessing version only), and the program will exit to the operating system. 


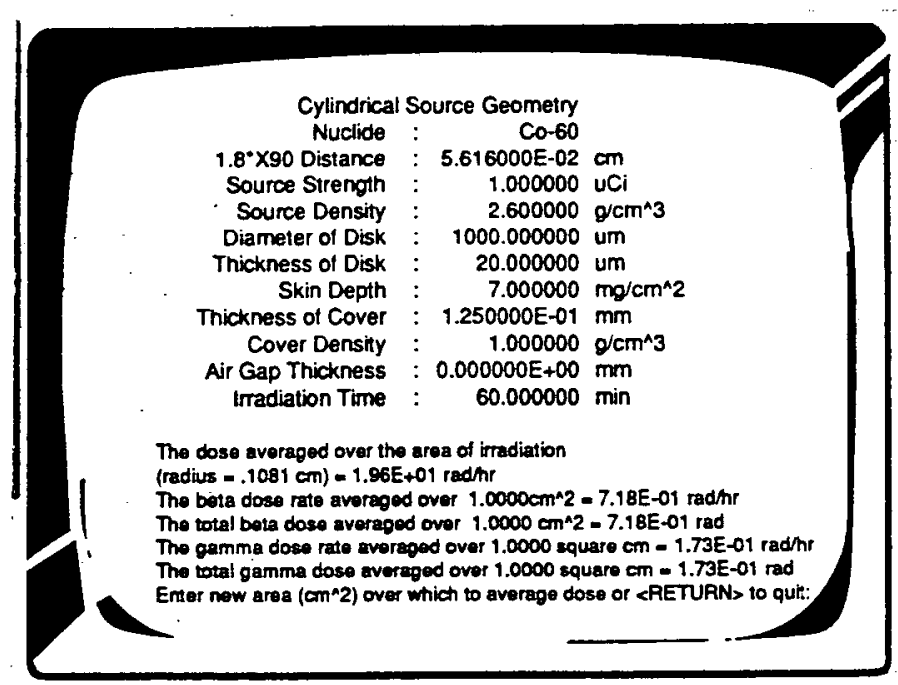

Figure 2.14 Output screen

VARSKIN Mod 2 can calculate doses from up to five radionuclides simultaneously. If more than one radionuclide is selected, the output screen and the output file, if requested, will display the sum of the beta doses and the sum of the gamma doses from all of the selected radionuclides. The sum of the total doses (gamma plus beta) from all of the selected radionuclides will also be displayed if any of the selected radionuclides contain a gamma component. The results are not shown in Figure 2.14.

\subsubsection{Output File}

\subsection{Running SADDE Mod 2}

If the user desires to save the data in an output file, a file is created by VARSKIN Mod 2 with a filename that conforms to the following procedure. VARSKIN Mod 2 attempts to open a file with the name VMOD2.OUT. If that file exists, VARSKIN Mod 2 attempts to open a file VMOD2A.OUT, followed by VMOD2B.OUT, etc. If all files including VMOD2Z.OUT already exist, VARSKIN Mod 2 will issue an error message and execution will be terminated.

The output file contains virtually the same information as displayed on the output screen. For all geometry options except for the three-dimensional slab, the output file will also contain the dose profile as a function of the radius of the circular skin irradiation area. This information is not provided when using the slab geometry since radial symmetry does not exist for a slab source.

At the DOS prompt, start SADDE Mod 2 by typing

\section{SADMOD2.}

The user will be asked to answer a series of questions, after which the new scaled absorbed dose distribution will be calculated. SADDE Mod 2 automatically accesses BETADATA.DAT and adds the new data, including the name of the radionuclide. 
In order to accomplish this task, however, BETADATA.DAT must be present in the same directory as SADDE Mod 2. If BETADATA.DAT is not present, the data generated by SADDE will be lost and the user must restart SADDE Mod 2.

Because the SADDE input is not printed in the output files and all computer users are prone to errors, the creation of an input file is highly recommended. The input file simply contains the answers to the questions asked by SADDE. The input file can be created using EDIT or EDLIN, text editors included in the DOS package, or a word processing program, as long as the input file is in ASCII format. The input file must contain the following information in the specified order:

1. The name of the radionuclide or mixture - This name must be no longer than six characters and must be different than any previous source name.

2. Enter "Y" if multiple radionuclides are to be included in the distribution. If not, enter " $N$ ".

If the response to step 2 is " $\mathrm{N}^{n}$, proceed to step 4.

3. Enter the number of radionuclides to be included in the distribution. This number must be less than 30 .

4. Enter the number of decay modes for the parent radionuclide.

5. Enter the atomic number of the transition product (daughter) nucleus (parent atomic number plus 1 ).

6. Enter the atomic mass of the decaying (parent) radionuclide.

If the response to step 2 is " $\mathrm{N}$ ", proceed to step 8.

7. Enter the relative weighting of the radionuclide. The sum of the weighting factors will usually equal to 1 .

8. Enter the endpoint energy of the decay path in $\mathrm{MeV}$.

9. Enter the probability (yield) of that decay path.

10. Enter the degree of forbiddenness for that decay path.

11. Repeat the information in steps 8 through 10 for each decay path.

12. Enter the number of Auger and/or internal conversion electrons emitted by this radionuclide. This number must be less than 30 .

13. Enter the energy of the Auger or internal conversion electron. 
14. Enter the yield of the Auger or internal conversion electron.

15. Repeat steps 13 and 14 for each Auger or internal conversion electron identified in step 12.

16. If multiple radionuclides are to be included, repeat steps 4 through 15 for each radionuclide.

Input data for parts 8, 9, and 10 are tabulated in Appendix A. Input data for steps 13 and 14 can be obtained from several references, including Kocher (1981). Examples of several input files are provided in Table 3. If the input file is named INPUT, for example, after changing directories or drives as discussed above, run the code by typing "SADMOD2 < INPUT". Note that if an input file is not used, the preceding list of input parameters must be supplied by the user in the order shown.

After completing the distribution calculations, SADDE MOD 2 automatically writes the results to the ASCII file BETADATA.DAT for use with VARSKIN Mod 2. If BETADATA.DAT is not present in the local subdirectory, SADDE Mod 2 will issue an error message and the code must be restarted. If BETADATA.DAT contains data for 100 radionuclides, then the user must edit BETADATA.DAT using either EDIT, EDLIN, or another word processor to remove any unwanted data entries. Note that a user can create a replacement BETADATA.DAT by creating an ASCII text file that contains the line

\section{ENDOFI}

SADDE Mod 2 will add the new distributions to this file, providing a customized BETADATA.DAT file.

Information needed for calculations using the gamma dose model in VARSKIN Mod 2 is not calculated by SADDE Mod 2. Therefore, SADDE Mod 2 simply enters values of 0.0 for both the average gamma energy and the specific gamma ray constant. See Sections 2.2.6 and 3.6 for more information concerning the gamma dose calculation. Section 3.6 also describes the methodology that can be used to add gamma dose information to BETADATA.DAT. 
VARSKIN Code Package

Table 3 Examples of input decks for SADDE Mod 2

\begin{tabular}{|c|c|c|}
\hline Input Deck for ${ }^{49} \mathrm{Ca}$ & Input Deck for ${ }^{90} \mathrm{Y}$ & Input Deck for ${ }^{49} \mathrm{Ca},{ }^{141} \mathrm{Ce}$, and ${ }^{90} \mathrm{Y}$ \\
\hline$\overline{\mathrm{Ca}-49}$ & Y-90 & $\operatorname{Mix} 1$ \\
\hline $\mathrm{n}$ & $\mathbf{n}$ & $\mathrm{y}$ \\
\hline 6. & 1 & 3 \\
\hline 21. & 40. & 6 \\
\hline 49. & 90. & 21. \\
\hline .525 & 2.2792 & 49. \\
\hline 0.0021 & .99984 & .333 \\
\hline 0 & 1 & .525 \\
\hline .77 & 0 & 0.0021 \\
\hline 0.0066 & & 0 \\
\hline 0 & Input Deck for ${ }^{90} \mathrm{Sr} /{ }^{90} \mathrm{Y}$ & .77 \\
\hline 1.1913 & SrY-90 & 0.0066 \\
\hline 0.07 & $\mathbf{y}$ & 0 \\
\hline 0 & 2 & 1.1913 \\
\hline 1.7467 & 1 & 0.07 \\
\hline 0.0018 & 39. & 0 \\
\hline 0 & 90. & 1.7467 \\
\hline 2.1789 & .500 & 0.0018 \\
\hline 0.915 & 1. & 0 \\
\hline 0 & 0.546 & 2.1789 \\
\hline 2.8915 & 1.000 & 0.915 \\
\hline 0.0041 & 1 & 0 \\
\hline 0 & 0 & 2.8915 \\
\hline \multirow[t]{2}{*}{0} & 1 & 0.0041 \\
\hline & 40. & 0 \\
\hline Input Deck for ${ }^{141} \mathrm{Ce}$ & 90. & 0 \\
\hline Ce-141 & .500 & 2 \\
\hline $\mathrm{n}$ & 1 & 59. \\
\hline 2 & 2.2792 & 141. \\
\hline 59. & .99984 & .333 \\
\hline 141. & 1 & 0.5813 \\
\hline 0.5813 & 0 & 0.2980 \\
\hline 0.2980 & & 0 \\
\hline 0 & & 0.4359 \\
\hline 0.4359 & & 0.702 \\
\hline 0.702 & & 0 \\
\hline 0 & & 2 \\
\hline 2 & & 0.1035 \\
\hline 0.1035 & & 0.188 \\
\hline 0.188 & & 0.1386 \\
\hline 0.1386 & & 0.0259 \\
\hline \multirow{8}{*}{0.0259} & & 1 \\
\hline & & 40. \\
\hline & & 90. \\
\hline & & .333 \\
\hline & & 2.2792 \\
\hline & , & .99984 \\
\hline & & 1 \\
\hline & & 0 \\
\hline
\end{tabular}




\subsection{Description of the Executable and Data Files}

During installation, a number of executable files will be copied from the distribution diskette to the target drive (hard disk or floppy diskette). The following discussion will give a brief overview of these various executable files and their function. VARSKIN Mod 2 will be discussed first, followed by SADDE Mod 2.

When the name of the batch file VARMOD2 is entered, a series of files is sequentially executed. The first file, BORDERB, simply draws a blue border around the text area of the screen and is included for aesthetic purposes only. The programs that draw borders around the text area of the screen will not affect a monochrome screen, and no border is drawn unless a math coprocessor is used. Next, the user interface (written in the programming language $\mathrm{C}$ ) is executed through the executable file INT.

Before the FORTRAN portion of VARSKIN Mod 2 is executed, BORDERR changes the color of the area bordering the text region of the screen to red, providing the math coprocessor package is used. This color change signifies that the user interface has been exited and the dose calculation portion of the program has been initiated. The calculational portion of the program has been given the name VARINT and is described in detail in Section 3. When the execution of VARINT is complete, the utility program BORDERBL erases the colored border that outlines the text portion of the screen and VARSKIN Mod 2 is complete. Note:

BORDERB, BORDERR, and BORDERBL can only be used with a math coprocessor. If the files under the subdirectory EMUL are installed, the border will remain black throughout the calculations.

Three other files are also required to run the VARSKIN Mod 2 package: BETADATA.DAT, OUTPUT.DAT, and HELP.TXT. The BETADATA.DAT file contains the necessary input for the source radionuclides available for VARINT. The OUTPUT.DAT file contains the data on the geometry package to be chosen by the user. When execution of the user interface portion of VARSKIN Mod 2 is completed, OUTPUT.DAT is saved on the disk. This file contains the input data needed by VARINT for execution. OUTPUT.DAT has another function, as well. When INT.EXE is executed, the default values are obtained from the latest version of OUTPUT.DAT. Thus, VARSKIN Mod 2 "remembers" the previous calculation. The HELP.TXT file contains the text portion of the help screens and can be edited to reflect the needs of the user. This file is used by the interface program INT.EXE.

SADDE Mod 2 consists of a single executable file, SADMOD2.EXE. The data file BETADATA.DAT must reside in the same directory as SADMOD2.EXE, or the data file cannot be modified. If the two files are not in the same directory, an error message will be generated by SADDE Mod 2 . 


\section{Technical Basis for VARSKIN}

Mod 2
This section discusses the theory underlying the dose calculation portion of VARSKIN Mod 2, including the modeling of beta dose, backscatter, protective clothing and air gaps, volume-averaged doses, offset particles, and hot particle gamma doses. A brief discussion of doses from internal conversion electrons is also provided. The formulas and features described in this section are incorporated into the FORTRAN program VARINT.

The computer code VARSKIN Mod 1 calculates skin dose rate from beta-emitters by performing a numerical integration of the Berger point kernel (Berger 1971). This kernel is mathematically written as

$$
B(r)=k E_{\beta} Y F_{\beta}\left(r_{1} / X_{90}\right) /\left(4 \pi \rho r^{2} X_{90}\right)
$$

where

$$
\begin{aligned}
r= & \text { a variable representing the distance between a source point and a } \\
& \text { dose point } \\
\mathbf{k}= & \text { a unit conversion constant } \\
\mathrm{E}_{\beta}= & \text { the average beta energy for the radionuclide } \\
\mathrm{Y}= & \text { the beta yield per disintegration } \\
\mathrm{F}_{\boldsymbol{\beta}}\left(\mathrm{r}_{1} / \mathrm{X}_{90}\right)= & \text { the scaled absorbed dose distribution as a function of the modified } \\
& \text { path length and the } \mathrm{X}_{90} \text { distance } \\
\mathrm{r}_{1}= & \text { the modified path length between the source point and dose point } \\
\rho= & \text { the density of the irradiated medium (assumed to be unity for tissue). }
\end{aligned}
$$

The $\mathrm{X}_{90}$ distance is defined as the radius of a sphere in which $90 \%$ of the beta energy is deposited from a point source in an infinite medium. A computer code, SADDE Mod 2, that calculates $F_{\beta}\left(r_{1} / X_{90}\right)$ for various radionuclides is described in Section 4.

In VARSKIN, the integration was performed over the area of the two-dimensional disk source. If the position at the skin depth from the center of the irradiated area is denoted as $\mathrm{d}$, then

$$
\overrightarrow{\mathbf{r}}=\mathbb{d}+\overrightarrow{\mathbf{r}}^{\prime}
$$

and

$$
\dot{D}(d)=\int_{0}^{2 \pi} \int_{0}^{R} S_{A} B\left(r^{\prime}, d\right) r^{\prime} d r^{\prime} d \theta
$$


where $R$ is the radius of the source disk, $d=d \cdot \hat{i}$, and $S_{A}$ is source term (assumed to be constant with respect to time and space for VARSKIN Mod 2 areal calculations).

For the purposes of illustration, the following discussion describes a dose calculation for the three-dimensional disk geometry shown in Figure 3.1. The integration is performed by choosing 26 dose points (points at which the dose rate is to be calculated), beginning at the source centerline and proceeding radially outward from the centerline at the skin depth level. Since the source is symmetric, dose points represent concentric isodose circles that describe the radial dose rate profile at a given depth in skin. For each dose point, an integration is performed over the area of the disk-shaped source by choosing eight concentric circles located at radially equidistant positions on the source. One of these circles defined by a radial dose point is chosen, subdivided into eight source points at equiangular locations (angular source points), and the dose rate at the dose point from each of these eight source points is calculated. The contribution to the dose from the first four points is then compared to the contribution to the last four points; if the relative difference between the two contributions is less than a user-selected tolerance (denoted as the relative error), then convergence of the integral for the circle is considered to be achieved and this procedure is repeated at the next radial position. If the relative difference between the two contributions is greater than the relative error, each of the two contributions is again subdivided into eight source points and the above procedure is repeated for each of the two sets. This process, known as the NewtonCotes eight-panel quadrature routine, provides a fast and accurate method of numerically integrating complex functions such as the Berger kernel.

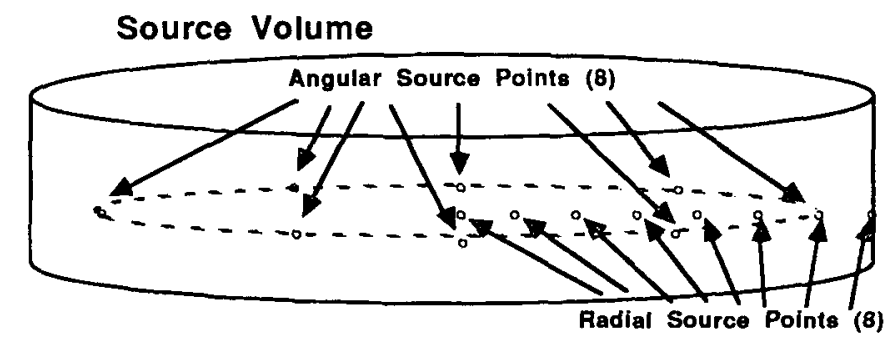

Dose area at the skin depth level

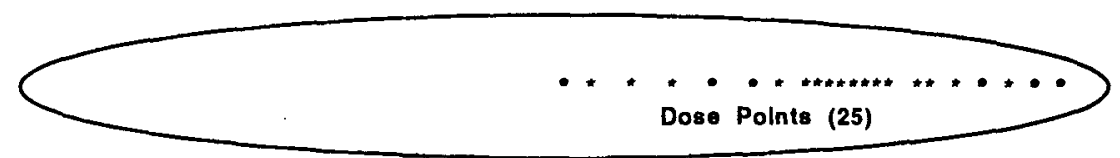

Figure 3.1 Schematic representation of a dose calculation for a three-dimensional disk source. Note that the $z$ value for the source is constant to reduce clutter. 
Once a dose rate value from each radial dose point is determined, the first four radial values are summed and compared to the sum of the last four radial values. These two groups of radial points are checked for convergence, and the above procedure is followed until convergence is reached for all radial points in the source. The dose rate at the next dose point is then calculated until values are obtained at all 26 dose points. If the dose rate profile defined by these 26 points as a function of target radius $d^{\prime}$ is denoted $\mathrm{D}\left(\mathrm{d}^{\prime}\right)$, then the dose rate averaged over an area in the skin is calculated using the equation

$$
D_{\text {avg }}=\frac{2 \pi \int_{0}^{R} D\left(d^{\prime}\right) d^{\prime} d d^{\prime}}{\pi R^{2}}
$$

The 26 dose points are chosen such that more points are chosen where the dose profile is changing the most. In general, this occurs at the edge of the source. The distribution of dose points is chosen automatically within VARSKIN Mod 2 and is listed in the output file.

VARSKIN Mod 2 uses the same Newton-Cotes eight-panel quadrature routines as VARSKIN with the addition of a third integration that accounts for the thickness of the source $T$. The eight-panel quadrature routine was chosen to optimize computer time and calculational accuracy. Thus, the dose integral is written

$$
\dot{D}(\mathrm{~d})=\int_{0}^{2 \pi} \int_{0}^{R} \int_{0}^{T} S_{v} B\left(r^{\prime}, d, \theta, z\right) r^{\prime} d z d r^{\prime} d \theta
$$

where $S_{v}$ is the volumetric source strength. A similar equation can be written for spherical and slab geometries. Those equations are:

$$
\dot{D}(d)=S_{v} \int_{0}^{\pi} \int_{0}^{2 \pi} \int_{0}^{R} B\left(r^{\prime} d^{\prime}, \theta, \phi\right) r^{\prime} \sin \theta d r^{\prime} d \theta d \phi
$$

in spherical geometry, and

$$
\dot{D}(d)=S_{v} \int_{-x_{\max }}^{x_{\max }} \int_{-y_{\max }}^{y_{\max }} \int_{0}^{\text {thichnoses }} B\left(d^{\prime}, x, y, z\right) d z d y d x
$$

in slab geometry. 
VARSKIN Technical Basis

\subsection{Backscatter}

When modelling a three-dimensional source, two types of attenuation must be addressed: attenuation due to the presence of the source material and attenuation due to geometry (i.e., $1 / \mathrm{r}^{2}$ attenuation). The VARSKIN Mod 2 code determines the attenuation within the source material by calculating the path lengths within the source, outside the source but above the skin, in the cover material (if any), and in the skin. Each of the path lengths is then modified by multiplying the path length by the ratio of the density of the material to the density of water.

VARSKIN Mod 2 incorporates a limited backscatter correction. When Berger developed the point kernel used in VARSKIN Mod 2, he used data that was measured in an infinite water medium. Therefore, the scaled absorbed dose distributions used by VARSKIN Mod 2 include backscatter from the surrounding medium. For infinitely thin sources (i.e., point sources or two-dimensional disk sources) that are bounded on one side by air (the side not in contact with the skin), the calculated doses overestimate the actual skin dose because they include the contribution from particles that were scattered from the medium behind the source toward the skin.

The absence of backscatter occurs only in the case of infinitely thin sources. For sources with a finite thickness, the greatest contribution to skin dose is from beta particles emitted from the portion of the source that is nearest to the skin. Because the remaining source material lies above this portion, backscatter from the remaining source material must be included. Since most hot particles have a density higher than that of water (such as fuel or stellite), the backscatter component is probably underestimated from the portion of the source that is nearest to the skin. This underestimate is compensated, within the accuracy of the calculations, by the overestimate of backscatter from the portions of the source that are farthest from the skin. Thus, for three-dimensional sources, a backscatter correction is not included in the code.

Backscatter calculations and their results for plane, isotropic sources at a skin density thickness of $7 \mathrm{mg} / \mathrm{cm}^{2}$ have been published by Cross et al. (1990). Figure 3.2 illustrates the backscatter factor as a function of $E_{\max }$ for a plane source and a point detector. Because of reciprocity, the same backscatter factor is applicable for a point source and a plane detector. Other data published by Cross et al. suggest that the backscatter factor for a plane source and a plane detector is within $10 \%$ of the data in Figure 3.2. The backscatter factor incorporated within VARSKIN Mod 2 is an extension of the Cross data.

Since the data from Cross only applies to point sources irradiating a $1 \mathrm{~cm}^{2}$ area or a $1 \mathrm{~cm}^{2}$ source irradiating a point, the backscatter correction is used sparingly. Backscatter corrections are performed only when the following conditions exist: 1) the source is either a point or an infinitely-thin plane, and 2) the area over which the dose is averaged is $1 \mathrm{~cm}^{2}$. For other conditions, no backscatter correction is made. 


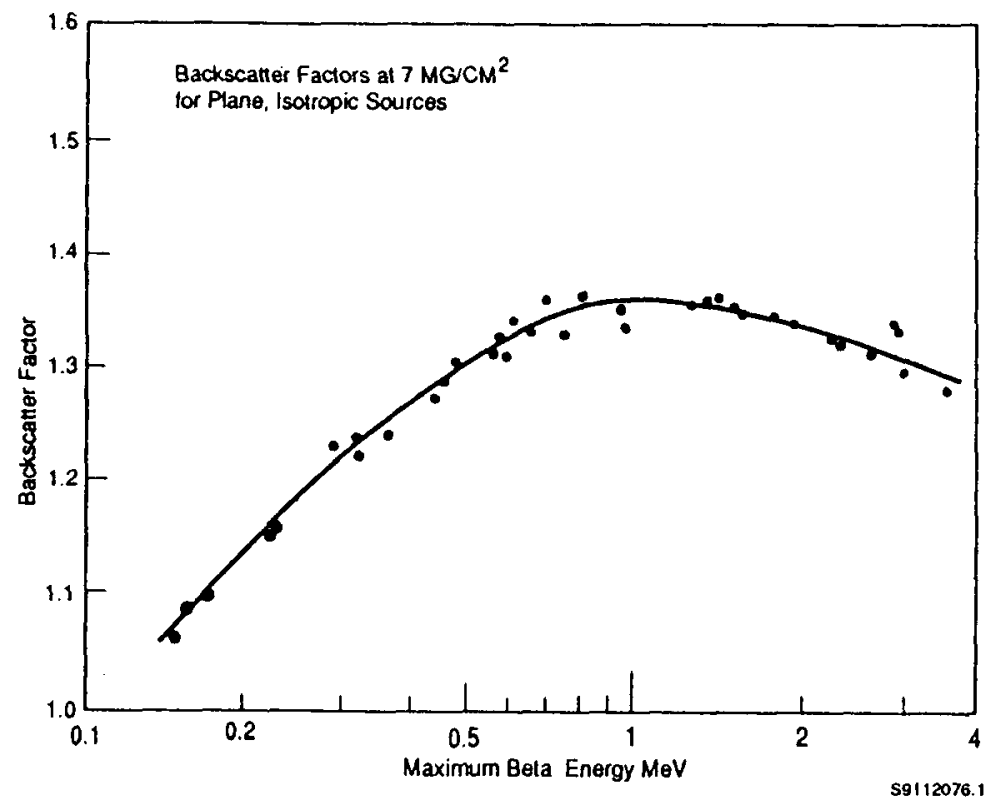

Figure 3.2 Backscatter correction factor at a skin depth of $7 \mathrm{mg} / \mathrm{cm}^{2}$ (Cross et al. 1990)

The backscatter factor incorporated into VARSKIN Mod 2 was derived in the following manner. The abscissa of Figure 3.2, $\mathrm{E}_{\max }$, was converted to $\mathrm{X}_{90}$ using the data presented by Berger (1971). The $\mathrm{X}_{90}$ distance was then divided by $0.007 \mathrm{~cm}$, the skin density thickness at which the Cross data was calculated, to obtain the backscatter factor shown in Figure 3.3. Implicit in the VARSKIN Mod 2 backscatter factor is the assumption that the shape of the curve in Figure 3.2 does not change with skin depth, but that the curve merely shifts to the left at depths less than $7 \mathrm{mg} / \mathrm{cm}^{2}$ and shifts to the right at depths greater than $7 \mathrm{mg} / \mathrm{cm}^{2}$.

A feature of the backscatter factor in VARSKIN Mod 2 is that backscatter is included with the protective clothing and air gap models and at different skin depths. Since the abscissa of Figure 3.3 is labeled " $\mathrm{X}_{90}$ Thickness", an equivalent thickness that includes the skin depth, the thickness of protective clothing, and the air gap thickness must be calculated. The equivalent thickness is obtained by adding the density thicknesses of skin, protective clothing, and any air gap and dividing by $1 \mathrm{~g} / \mathrm{cm}^{2}$. The $X_{90}$ distance is then divided by the equivalent thickness and the backscatter factor obtained from Figure 3.3. 


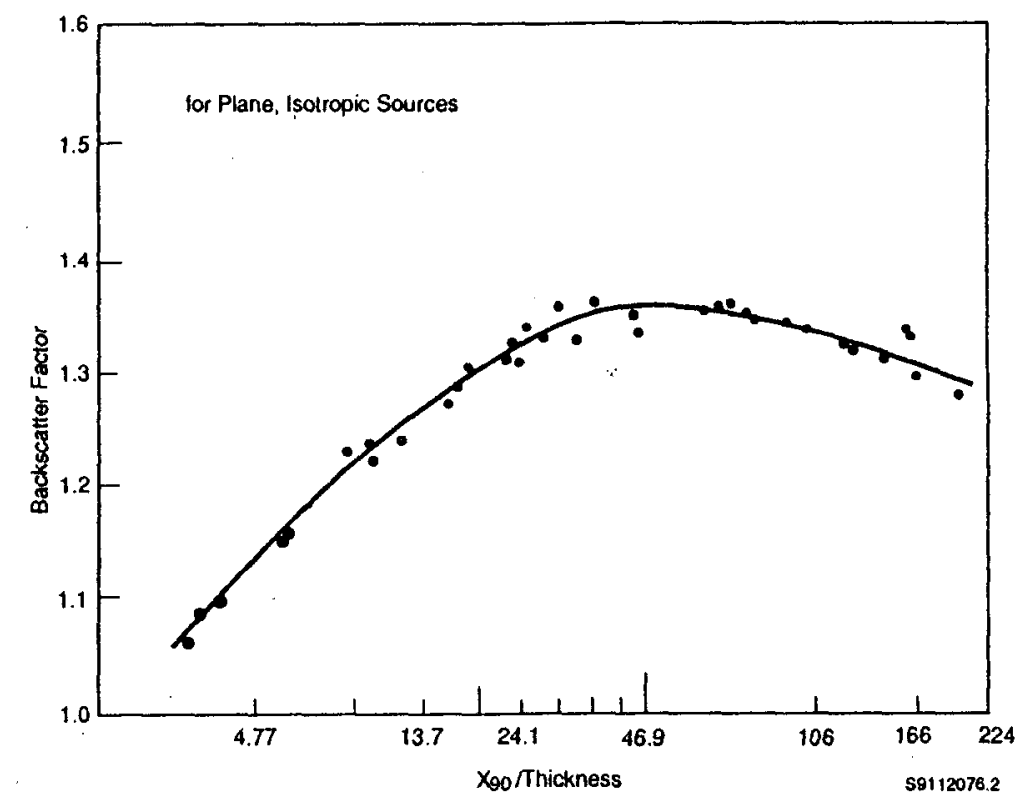

Figure 3.3 Backscatter model as a function of $\mathrm{X}_{90}$ /thickness based on Cross's backscatter model (Cross et al. 1990)

The original VARSKIN document (Traub et al. 1987) included a table of dose factors for various radionuclides. In VARSKIN Mod 2, these values have changed significantly because the following have been added: a backscatter factor, new low-energy electron contributions to dose (see Chapter 4), upgraded radioactive decay data, dose from Auger and internal conversion electrons, and gamma dose. The updated dose factors are presented in Table 4. The dose factors in Table 4 were calculated using the computer code VARSKIN Mod 2 using a point source of strength $1 \mu \mathrm{Ci} / \mathrm{cm}^{2}$ at a depth of $7 \mathrm{mg} / \mathrm{cm}^{2}$. The dose factors provide the dose rate in $\mathrm{rad} / \mathrm{h}$. The dose factors in Table 4 do reflect any contribution from gamma components of the radionuclide.

Many users who are familiar with the original VARSKIN code may wish to repeat their original calculations using VARSKIN Mod 2. Since the backscatter model is automatically incorporated into VARSKIN Mod 2, doses calculated with the two versions will differ. To perform original VARSKIN calculations using VARSKIN Mod 2, the user should choose the three-dimensional disk model with a source diameter of $1 \mu \mathrm{m}$, a source thickness of $1 \mu \mathrm{m}$, and a source density of $0.001 \mathrm{~g} / \mathrm{cm}^{3}$. By choosing a three-dimensional source geometry, the backscatter model will not be included and original VARSKIN calculations can be reproduced. 
VARSKIN Technical Basis

Table 4. Dose factors ${ }^{(a)}$

\begin{tabular}{|c|c|c|c|c|c|}
\hline$\underline{\text { Radionuclide }}$ & Factor & $\underline{\text { Radionuclide }}$ & Factor & $\underline{\text { Radionuclide }}$ & Factor \\
\hline \multirow[t]{2}{*}{${ }^{3} \mathrm{H}$} & $-0-$ & ${ }^{63} \mathrm{Ni}$ & $-0-$ & ${ }^{124} \mathrm{I}$ & 0.535 \\
\hline & & & & ${ }^{13} 1_{I}$ & 5.03 \\
\hline${ }^{11} \mathrm{C}$ & 6.35 & ${ }^{64} \mathrm{Cu}$ & 0.241 & ${ }^{132} \mathrm{I}$ & 5.92 \\
\hline \multirow[t]{2}{*}{${ }^{14} \mathrm{C}$} & 0.942 & & & & \\
\hline & & ${ }^{6} \mathrm{Zn}$ & 0.0669 & ${ }^{133} \mathrm{Xe}$ & 3.71 \\
\hline \multirow{2}{*}{${ }^{13} \mathrm{~N}$} & 6.41 & & & & \\
\hline & & ${ }^{68} \mathrm{Ga}$ & 5.57 & ${ }^{137} \mathrm{Cs}$ & 5.78 \\
\hline${ }^{15} \mathrm{O}$ & 6.59 & ${ }^{76} \mathrm{As}$ & 6.37 & ${ }^{140} \mathrm{Ba}$ & 5.32 \\
\hline \multirow[t]{2}{*}{${ }^{18} \mathrm{~F}$} & 5.96 & & & & \\
\hline & & ${ }^{82} \mathrm{Br}$ & 4.65 & ${ }^{140} \mathrm{La}$ & 5.89 \\
\hline${ }^{22} \mathrm{Na}$ & 5.02 & & & & \\
\hline \multirow[t]{2}{*}{${ }^{24} \mathrm{Na}$} & 6.20 & ${ }^{79} \mathrm{Kr}$ & 0.173 & ${ }^{141} \mathrm{Ce}$ & 5.79 \\
\hline & & ${ }^{85} \mathrm{Kr}$ & 5.45 & ${ }^{144} \mathrm{Ce}$ & 3.22 \\
\hline \multirow[t]{2}{*}{${ }^{28} \mathrm{Mg}$} & 4.94 & & & & \\
\hline & & ${ }^{86} \mathrm{Rb}$ & 6.13 & ${ }^{144} \mathrm{Pr}$ & 6.52 \\
\hline \multirow[t]{2}{*}{${ }^{28} \mathrm{Al}$} & 6.68 & & & & \\
\hline & & ${ }^{89} \mathrm{Sr}$ & 6.01 & ${ }^{147} \mathrm{Pm}$ & 2.15 \\
\hline \multirow[t]{2}{*}{${ }^{32} \mathrm{P}$} & 6.24 & ${ }^{90} \mathrm{Sr}$ & 5.25 & & \\
\hline & & & & ${ }^{198} \mathrm{Au}$ & 5.89 \\
\hline \multirow[t]{2}{*}{${ }^{35} \mathrm{~S}$} & 1.04 & "87 $Y$ & 0.155 & & \\
\hline & & ${ }^{88} \mathrm{Y}$ & 0.274 & ${ }^{203} \mathrm{Hg}$ & 4.60 \\
\hline${ }^{36} \mathrm{Cl}$ & 5.45 & ${ }^{90} \mathrm{Y}$ & 6.40 & & \\
\hline${ }^{38} \mathrm{Cl}$ & 6.55 & ${ }^{91} \mathrm{Y}$ & 6.01 & ${ }^{210} \mathrm{~Pb}$ & 0.061 \\
\hline${ }^{40} \mathrm{~K}$ & 4.87 & ${ }^{95} \mathrm{Zr}$ & 4.10 & ${ }^{204} \mathrm{~T} 1$ & 5.43 \\
\hline \multirow[t]{2}{*}{${ }^{42} \mathrm{~K}$} & 6.54 & & & ${ }^{200} \mathrm{~T} 1$ & 6.90 \\
\hline & & ${ }^{95} \mathrm{Nb}$ & 0.885 & & \\
\hline${ }^{45} \mathrm{Ca}$ & 2.85 & & & ${ }^{212} \mathrm{~Pb}$ & 9.90 \\
\hline${ }^{47} \mathrm{Ca}$ & 5.61 & 99 Mo & 5.59 & ${ }^{214} \mathrm{~Pb}$ & 8.73 \\
\hline \multirow[t]{2}{*}{${ }^{49} \mathrm{Ca}$} & 6.43 & & & & \\
\hline & & ${ }^{99} \mathrm{Tc}$ & 3.15 & ${ }^{210} \mathrm{Bi}$ & 5.74 \\
\hline${ }^{47} \mathrm{Sc}$ & 4.77 & & & ${ }^{212} \mathrm{Bi}$ & 2.60 \\
\hline${ }^{49} \mathrm{Sc}$ & 6.34 & $\begin{array}{l}{ }^{103} \mathrm{Ru} \\
{ }^{106} \mathrm{Ru}\end{array}$ & $\begin{array}{l}2.56 \\
-0-\end{array}$ & ${ }^{214} \mathrm{Bi}$ & 5.98 \\
\hline \multirow[t]{2}{*}{${ }^{52} \mathrm{Mn}$} & 0.898 & & & ${ }^{228} \mathrm{Ac}$ & 6.64 \\
\hline & & ${ }^{111} \mathrm{Ag}$ & 5.72 & & \\
\hline \multirow[t]{2}{*}{${ }^{59} \mathrm{Fe}$} & 3.99 & & & ${ }^{231} \mathrm{Th}$ & 3.53 \\
\hline & & ${ }^{124} \mathrm{Sb}$ & 5.12 & ${ }^{234 \mathrm{~m}} \mathrm{~Pa}$ & 6.29 \\
\hline${ }^{56} \mathrm{Co}$ & 5.10 & ${ }^{125} \mathrm{Sb}$ & 2.49 & & \\
\hline${ }^{58} \mathrm{Co}$ & 0.259 & & & ${ }^{234} \mathrm{Th}$ & 1.50 \\
\hline${ }^{60} \mathrm{Co}$ & 3.89 & ${ }^{132} \mathrm{Te}$ & 2.65 & & \\
\hline
\end{tabular}

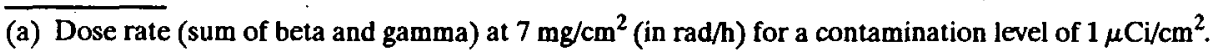




\subsection{Protective Clothing and Air Gap Models}

The backscatter model is executed automatically when either the point source or two-dimensional disk source models are chosen. However, the backscatter model is only applied to a dose area of $1 \mathrm{~cm}^{2}$. No additional information is required from the user.

Both protective clothing and air gaps can be modelled using VARSKIN Mod 2. The models use the concept of path length to determine the energy lost in either clothing or air. The "path length" is not the true path traversed by the beta particle; it is merely a mathematical convenience introduced to provide a measure of the energy lost in each layer.

The method used to determine path length within the source and within the cover material can be illustrated using Figure 3.4. For the pictured cylindrical or threedimensional disk source, the known values in the figure are the source radius $(R M A X)$, the horizontal distance from the centerline to the source point (SRAD), the source thickness (STHICK), the cover thickness (CTHICK), the skin depth $(S D E P)$, the source and cover densities ( $\rho_{\mathrm{s}}$ and $\rho_{\mathrm{c}}$, respectively), the distance from the centerline to the dose point $(P S)$, and the distance from the skin to the plane of the source point (DRAD). The quadrature routines choose values for the distance from the centerline to the source point (SRAD), the angle between SRAD and PS (THETA), and the height of the dose point (DRAD). The first quantity to be calculated is $r$, the physical distance from a source point to a dose point. To do this, the square of the projected distance, $r_{p}{ }^{2}$, is calculated using the law of cosines:

$$
\mathbf{r}_{\mathrm{p}}^{2}=\mathrm{PS}^{2}+\mathrm{SRAD} \mathrm{D}^{2}-2 \cdot \mathrm{PS} \cdot \mathrm{SRAD} \cdot \cos (\mathrm{THETA})
$$

The value of $r$ can then be determined using the Pythagorean Theorem:

$$
\mathbf{r}=\sqrt{\mathbf{r}_{\mathrm{p}}^{2}+\mathrm{DRAD}^{2}}
$$

The quantity $r$ is used in the denominator of the expression in Equation (3.1) and represents the geometric attenuation between the dose point and the source point. This quantity is further analyzed to calculate the modified path length used to evaluate the scaled absorbed dose distribution as discussed below. 


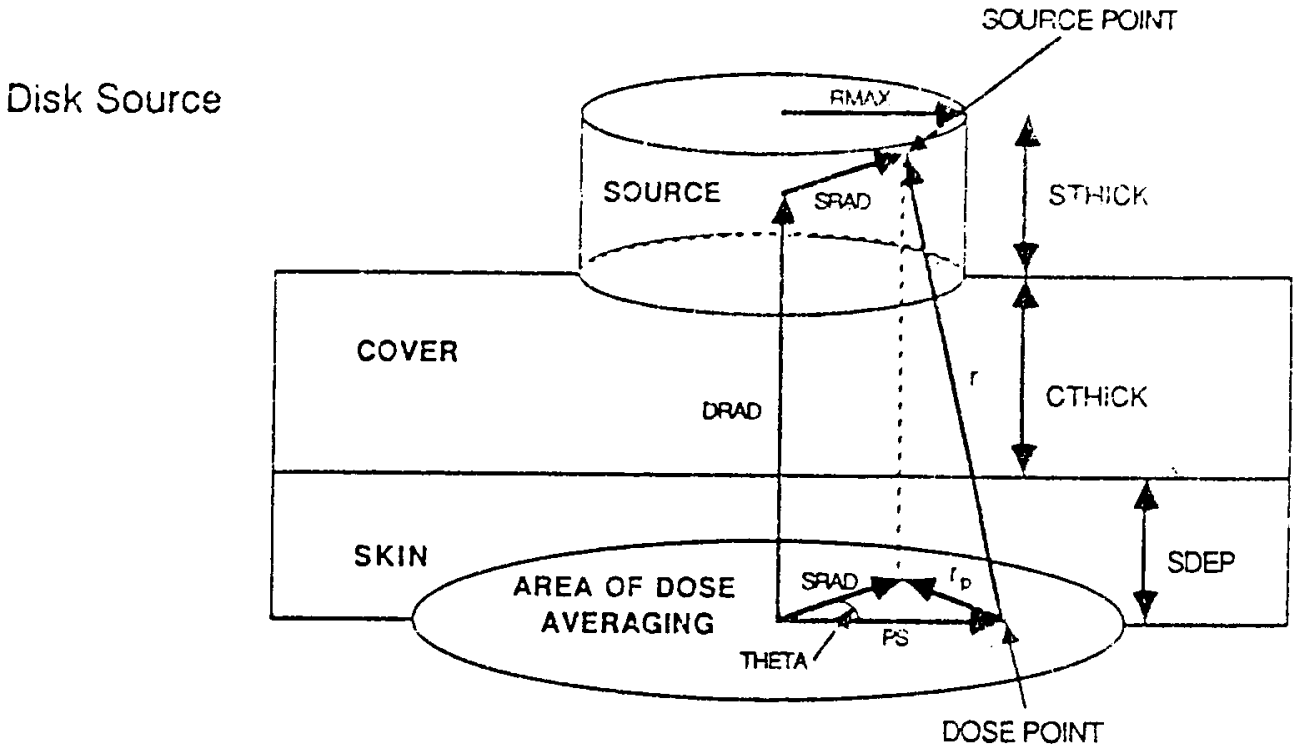

Figure 3.4 Schematic drawing of a generic dosed calculation performed by VARSKIN Mod 2 for the three-dimensional disk geometry

By the law of similar triangles, the ratio to $r$ of each of the actual distances along $r$ through the source, the cover material, and the tissue is the same as the ratio to $D R A D$ of the thickness of the cover material, the tissue, and the remaining distance along $D R A D$, respectively, providing that the line connecting the dose point and the source point exits through that part of the source that is in contact with the cover material. Thus, the distance traveled through the cover material is written

$$
t_{c}=C T H I C K \cdot r / D R A D
$$

The distance traveled through the skin is given by

$$
\mathbf{r}_{\mathbf{t}}=\operatorname{SDEP} \cdot \mathbf{r} / \mathrm{DRAD}
$$

Finally, the distance traveled through the source is given by

$$
r_{s}=(D R A D-C T H C K-S D E P) \cdot r / D R A D
$$




$$
r_{1}=\left(r_{s} \rho_{s}+r_{c} \rho_{c}+r_{t} \rho_{t}\right) / \rho_{t}
$$

where $\rho_{t}$ is the density of tissue (water).

For small-diameter sources, the path between the dose point and the source point may pass through the side of the source, that is, the path may exit the sources and pass through air before passing into skin. Thus, the quantity in Equation (3.7) must be further analyzed to determine the path length within the source and the path length outside the source but above the cover-material level. The actual path length within the source is multiplied by the source density, and the path length outside the source and above the cover material is multiplied by the density of the material outside the source, assumed to be air.

In spherical geometry, the actual distance from source point to dose point is given by Equation (3.8) after substituting SRAD SIN $\phi$ for SRAD. In slab geometry, the actual distance is given by the equation

$$
\begin{aligned}
r= & {\left[\left(x_{\text {source }}-x_{\text {dose }}\right)^{-2}+\left(y_{\text {source }}-y_{\text {dosd }}\right)^{2}\right.} \\
& \left.+\left(z_{\text {source }}-z_{\text {dose }}\right)^{2}\right]^{1 / 2}
\end{aligned}
$$

Equations (3.9) through (3.13) then apply to both spherical and slab geometries.

\subsection{Volume- Averaged Dose Model}

The volume-averaged dose model, shown schematically in Figure 2.9, allows the calculation of doses averaged over volumes of skin. The volume is bounded by any two planes of irradiated skin, i.e., any two planes between zero skin depth and the skin depth that corresponds to the maximum penetration of beta particles emitted by the source. For sources in contact with the skin, the maximum penetration depth is equal to $1.8 \cdot \mathrm{X}_{90}$. Doses averaged over $1 \mathrm{~cm}^{2}$ are calculated at 20 skin depths between these two limits, and a cubic spline (a third-order piecewise polynomial curve fit) is fit to this depth-dose distribution. When the user enters the skin depths corresponding to the volume of interest, VARSKIN Mod 2 integrates the depth dose function over the region of interest to obtain the volume-averaged dose. Note that the doses calculated at the various skin depths are averaged over $1 \mathrm{~cm}^{2}$ when VARSKIN Mod 2 is first invoked. The user can choose to calculate volumeaveraged doses for different areas only after calculating volume-averaged doses for $1-\mathrm{cm}^{2}$ areas.

The volume-averaged dose model requires roughly 20 times the execution time as a single depth calculation to establish the depth dose function. Once this function is calculated, however, subsequent calculations require less than 1 second. Thus, doses 


\subsection{Offset Particle Model}

\subsection{Hot Particle Gamma Dose Model}

averaged over a number of different volumes can be obtained in roughly the same amount of time as the dose averaged over a single volume.

The offset particle model, shown schematically in Figure 2.13, allows calculation of skin dose averaged over areas that are not directly below the contaminant. This model is intended to be used to calculate doses from multiple hot particles. In the interests of reasonable execution time and code complexity, the offset particle model is available only for the three-dimensional slab geometry.

The offset particle model requires only one input variable, the distance of the offset. When performing the calculations for the model, VARSKIN Mod 2 assumes that the offset is in the $x$ direction. Since this requirement implies a symmetrical source, the $x$ - and $y$-side lengths must be equal. If a user invokes the offset particle model with a source that does not have equal $x$ - and $y$-side lengths, VARSKIN Mod 2 will request that the user re-enter the $x$ - and $y$-side lengths. VARSKIN Mod 2 also provides the user with the $x$-side length that corresponds to a source of equivalent area should the user wish to maintain the area of the source.

For multiple particle irradiations, doses from each particle must be calculated separately, running VARSKIN Mod 2 for each particle. The offset particle model does not calculate the maximum dose to skin from several particles; the iterative process for determining the maximum dose to $1 \mathrm{~cm}^{2}$ is outlined in Section 5.3 .

Hot particle gamma doses calculated by VARSKIN Mod 2 use a simplified model that has limited application. The model was developed by Lantz and Lambert (1990). The gamma dose model assumes that the source is a point source and that any material between the source and skin is nearly tissue-equivalent. Therefore, the largest dimension of any source has been arbitrarily limited to a maximum of $1 \mathrm{~mm}$. Also, the size of any air gap is limited to $5 \mathrm{~cm}$. The gamma dose is calculated (provided that the radionuclide emits gamma radiation) only for a skin area of $1 \mathrm{~cm}^{2}$. The gamma dose model has been experimentally verified by Durham and Lantz (1991).

Two pieces of information are required to perform the gamma dose: the specific gamma-ray exposure constant and the average gamma energy. The specific gammaray constant is used to determine gamma doses when electronic equilibrium is fully established. The average gamma energy is used to determine the extent to which electronic equilibrium is established.

The specific gamma-ray exposure constant, in units of $\mathrm{R}-\mathrm{cm}^{2}-\mathrm{Ci}^{-1}-\mathrm{h}^{-1}$, was calculated for the radionuclides listed in BETADATA.DAT using data published by Unger and Trubey (1982). This reference also contains all of the information needed to calculate the specific gamma ray constants for other gamma-emitting radionuclides.

To calculate the specific gamma-ray exposure constant, the following equation should be used (Attix 1986): 
VARSKIN Technical Basis

$$
\Gamma=193.8\left[\sum_{i=1}^{n} P_{i} E_{i}\left(\frac{\mu_{e n}}{\rho}\right)_{E_{i, a i r}}\right]
$$

where $P_{i}$ is the probability of emission of a gamma ray having an energy $E_{i}$ in $\mathrm{MeV}$ and $\left(\mu_{\mathrm{en}} / \rho\right)_{E \mathrm{i}, \text { air }}$ is the mass energy absorption coefficient in air in units of $\mathrm{m}^{2} / \mathrm{kg}$ for the gamma ray. Data for $\left(\mu_{\mathrm{en}} / \rho\right)_{\text {Ei,air }}$ can be obtained from Attix (1986), and data for $E_{i}$ and $P_{i}$ can be found in Unger and Trubey (1982).

The average gamma energy in $\mathrm{keV}$ was calculated using the equation

$$
E_{\text {avg }}=\frac{\sum_{i} P_{i} E_{i}}{\sum_{i} P_{i}}
$$

The specific gamma-ray constant and the average gamma energy are included in BETADATA.DAT. Beta-dose data for radionuclides not included in BETADATA.DAT can be added using the computer code SADDE Mod 2, but data needed for calculating gamma dose cannot be added using this code. It is possible for a user to add gamma dose data to BETADATA.DAT by manually editing the last line for the radionuclide. After adding a radionuclide using SADDE Mod 2, the last line of the added data contains two zeros. The user can replace the first 0.0 with the correct value for the specific gamma-ray exposure constant and the second 0.0 with the value of the average energy. VARSKIN Mod 2 will then automatically calculate the gamma component of dose when the radionuclide is chosen.

Similarly, the data in BETADATA.DAT can be modified to calculate doses from radionuclides that are pure gamma emitters. This can be accomplished by copying the lines of data for any of the listed radionuclides using a suitable editor. The first line of data (after the radionuclide name, which should be changed to reflect the new radionuclide) contains the values of the average beta energy, the $\mathrm{X}_{90}$ distance, and the beta yield of the radionuclide. Substituting a value of " $0.1^{\text {" for the }} \mathrm{X}_{\mathbf{9 0}}$ distance will cause VARSKIN Mod 2 to bypass the beta dose calculation. The appropriate values for the specific gamma ray exposure constant and the average gamma energy, respectively, can be substituted for the two values in the last line of data. Note that the format of the data must be preserved. 


\subsection{Internal} Conversion and Auger Electrons

\subsection{Positron- Emitting Radionuclides}

The input file for VARSKIN Mod 2, BETADATA.DAT, includes the contribution to the scaled absorbed dose distribution function, $F_{\beta}$, from Auger and internal conversion electrons. BETADATA.DAT includes the contribution to $F_{\beta}$ from all Auger and internal conversion electrons that have energies greater than $65 \mathrm{keV}$ and emission yields, relative to the beta emission yield, of greater than 0.005 . The energy cutoff was chosen since electrons with energies less than $65 \mathrm{keV}$ do not contribute to dose at a depth of $7 \mathrm{mg} / \mathrm{cm}^{2}$. The emission yield cutoff was chosen because the contribution to dose from electrons with relative yields below 0.005 is negligible. Due to the integration method used in VARSKIN Mod 2, it is not possible to calculate skin dose from radionuclides that emit Auger and internal conversion electrons without an accompanying beta spectrum.

Inclusion of Auger and internal conversion electrons with energies or yields below the cutoff value can be accomplished by using SADDE Mod 2. A description of the methodology used to include Auger and internal conversion electrons is included in Section 4.2.

Radionuclides that emit positrons pose a problem when using VARSKIN Mod 2 and SADDE Mod 2, since all positrons are treated as negatrons. The energy distribution of positrons is higher than that of negatrons because of coulombic repulsion by the nucleus. Thus, positrons have a higher ratio of average energy to maximum energy than negatrons. Also, $F_{\beta}$ is skewed toward higher positrons than negatrons.

Average energies calculated for positrons using SADDE Mod 2 were compared to published data for positrons (Kocher 1981). It was found that the difference in the average energy calculated using SADDE Mod 2 was consistently less than the published value by as much as $31 \%$. Thus, the values of the average energy for positron-emitting radionuclides were adjusted to remain consistent with values of average energy generated using RADLST (Burrows 1988).

The adjustment to the average energy will not correct differences in the spatial energy distribution for positron-emitting radionuclides. Care must be exercised when performing calculations of skin dose from positrons using VARSKIN Mod 2. Errors in the spatial energy distribution, $F_{\beta}$ range from $3 \%$ for low- $Z$ radionuclides to $31 \%$ for high- $Z$ radionuclides. However, errors in doses calculated for positrons will not be as large as the errors in $F_{\beta}$, especially if doses are averaged over $1 \mathrm{~cm}^{2}$. The errors associated with skin dose calculations for positrons will be consistent with the errors associated with the calculation, especially if the average energy, as calculated using SADDE Mod 2, is adjusted to be consistent with published data. 


\section{Technical Basis for SADDE}

\section{Mod 2}

\subsection{Beta Spectra}

This chapter presents the technical approach used in SADDE Mod 2. Much of the information is reprinted from the original NUREG document that describes SADDE (Reece et al. 1989) with additions that reflect the modifications. Section 2.3 highlights the modifications incorporated into the upgraded code, and this chapter serves as a complete technical description of SADDE MOD 2.

From the basic definitions of specific absorbed fraction for monoenergetic electrons given by Berger (1971), the absorbed dose rate is defined as follows:

$$
R\left(x, E_{0}\right)=A n k E_{0} \phi\left(x, E_{0}\right)
$$

where $\mathrm{R}\left(\mathrm{x}, \mathrm{E}_{0}\right)=$ the absorbed dose rate

$x=$ the distance from the point source

$A=$ the source activity

$\mathrm{n}=$ the number of electrons emitted at energy $\mathrm{E}_{0}$ per unit of source activity

$\mathbf{k}=\mathbf{a}$ unit conversion factor

$E_{0}=$ the initial energy of the electron

$\Phi\left(\mathrm{x}, \mathrm{E}_{0}\right)=$ the specific absorbed fraction of the energy.

For a spectrum of energies such as would be emitted from a source undergoing beta decay, the absorbed dose rate $R_{B}(x)$ would be:

$$
R_{\beta}(x)=A Y_{\beta} k \int_{0}^{E_{\max }} E S(E) \phi(x, E) d E
$$

where $E_{\max }$ is the endpoint energy of the beta spectrum, $Y_{B}$ is the number of beta particles emitted per disintegration (the yield), and $S(E)$ is the energy spectrum of the beta particles in particles/MeV.

For convenience, Equation (4.2) can be cast into the same form as Equation (4.1):

$$
R_{\beta}(x)=A Y_{\beta} k E_{a v} \phi_{\beta}(x)
$$

where $E_{a v}$, the mean energy of the spectrum, is defined as 


$$
E_{\mathrm{av}}=\frac{\int_{0}^{E_{\max }} E S(E) d E}{\int_{0}^{E_{\max }} S(E) d E}
$$

and

$$
\phi_{\beta}(x)=\int_{0}^{E_{\max }}\left(E / E_{a v}\right) S(E) \phi(x, E) d E
$$

The first step in determining the scaled absorbed dose distributions for a given isotope is calculating the emitted beta spectra. This is accomplished by a code called BETA, developed at Kansas State University by Richard Faw and Gale Simons, which (with minor modifications) is installed as a subroutine in the SADDE MOD 2 code. The BETA code is used with the permission of Faw and Simons. The following information is needed to calculate the beta spectrum from the decay of a radionuclide or mixture:

- the name of the radionuclide or mixture - This name must be no longer than six characters and must be different than any previous radionuclide name.

- an indication if multiple radionuclides are to be used when calculating the distribution

- if multiple radionuclides are to be included in the distribution, the number of those radionuclides - This number must be less than 30 .

- the number of decay modes for the parent radionuclide

- the atomic number of the transition product (daughter) nucleus

- the atomic mass of the decaying (parent) radionuclide

- if multiple radionuclides are to be included in the distribution, the relative weight of each radionuclide - The sum of the weighting factors will usually be equal to 1 .

- the endpoint (maximum) energy of the particular decay path 
- the probability of the particular decay path (the yield)

- the degree of forbiddenness for the particular decay.

If multiple radionuclides are to be included in the distribution, the last seven items must be repeated for each radionuclide.

Using this input data, the routine BETA calculates a normalized beta spectrum, $n(E)$, such that

$$
\int_{0}^{E_{\operatorname{mex}}} n(E) d E=Y_{\beta}
$$

The calculated spectrum is divided into 150 energy bins and then interpolated to 1500 values using a spline interpolation routine. These interpolated values are stored in an array called COMP (see Appendix $E$ for a listing of the FORTRAN programs). The average energy of the composite spectrum is calculated using the following algorithm:

$$
\mathrm{EAV}=\int_{0}^{\mathrm{E}_{\max }} \operatorname{COMP}(\mathrm{E}) \mathrm{E} d \mathrm{E}
$$

where $E_{\max }$ is the maximum endpoint energy for the composite spectrum.

The subroutine SADD uses the average energy and COMP to calculate the scaled absorbed dose function, $\Phi_{B}(x)$. The SADD routine determines the maximum beta range and sets the range step size to $1 / 150$ of this maximum range. The scaled absorbed dose function for the beta spectrum is calculated for each distance step by integrating through energy in steps of $1 / 150$ th of the maximum energy using the following equation:

$$
\phi_{B}=\frac{1}{E_{a v}} \int_{0}^{\mathrm{E}_{\max }} \mathrm{En}(\mathrm{E}) \phi(x, \mathrm{E}) \mathrm{dE}
$$

where $\Phi(\mathrm{x}, \mathrm{E})$ is the scaled absorbed dose function for monoenergetic electrons.

The function $\Phi(\mathrm{x}, \mathrm{E})$ is determined within the SADD routine by a routine named SPENS, an algorithm for interpolating Spencer's data (1959) for scaled absorbed dose distribution as a function of distance and electron energy through energy and 
distance. When SADD picks an energy (E) and a distance $(\mathrm{x}), \boldsymbol{\Phi}(\mathrm{x}, \mathrm{E})$ is calculated by the following algorithm:

$$
\Phi(\mathrm{x}, \mathrm{E})=\mathrm{F}(\xi, \mathrm{E}) / 4 \pi \rho \mathrm{x}^{2} \mathrm{X}_{90}(\mathrm{E})
$$

where $\xi$ is $x / X_{90}(E)$ and $\rho$ is the density of the absorbing medium.

$F(\xi, E)$ is a tabulated function of $\xi$ and $E$ that is interpolated to calculate the function value. Output of the routine SADD is the function $\Phi_{B}(x)$, the array PHI in the FORTRAN program, which has 150 values.

The $\mathrm{X}_{90}$ distance is calculated using the array PHI and EAV. This is accomplished by summing up the values of $\mathrm{dE} / \mathrm{dx}$ over distance and arriving at the total energy in the beta spectrum:

$$
\frac{d E}{d x}=4 \pi \rho x^{2} E_{a v} \Phi_{\beta}(x)
$$

Therefore,

$$
E_{T o t}=\sum_{i} \Delta x_{i} 4 \pi r x^{2} E_{a v} \Phi_{\beta}(x)
$$

Once the total energy in the spectrum is found, a second sum is computed as follows:

$$
\text { FRAC }=\sum_{i} \Delta x_{i} 4 \pi \rho x^{2} E_{a v} \Phi_{\beta}(x) / E_{t o t}
$$

FRAC is evaluated for successively larger values of $x$ until FRAC is 0.9 , then the current $x$ value becomes the $X_{90}$ value and is stored as the FORTRAN variable $X 90$.

The routine FBETA calculates the function $F_{B}(\xi)$. The routine uses $X 90$ and PHI to perform the following calculation:

$$
F_{\beta}(\xi)=4 \pi \rho x^{2} X_{90} \Phi_{\beta}(x)
$$

The values of $x$ are divided by $X_{90}$ and then $F_{B}(x)$ is tabulated against the $x$ values. The resulting values of $F_{B}(\rho)$ are stored in the array $F$. The final routine FIT uses 
linear interpolation to extract the 30 specific values of $F_{B}(\rho)$ needed by VARSKIN and writes these values to the output file.

Values of the functions $F(\xi, E)$ and $X_{90}(E)$ in Equation (4.9) have been extended to energies as low as $0.05 \mathrm{keV}$ in SADDE Mod 2. Work performed by Wilson and Reece (1991) extends the values of these functions to $0.05 \mathrm{keV}$. Wilson's results compared favorably with Berger's improved point kernels (1973) at $25 \mathrm{keV}$, the lowest energy for which Berger's functions were provided. The low energy corrections in SADDE Mod 2 should have little bearing on doses averaged over areas larger than a few $\mathrm{mm}^{2}$, but doses averaged over smaller areas could be affected, particularly if the contamination is in contact with skin.

\subsection{Auger and} Internal Conversion Electrons
The contribution to dose from Auger and internal conversion electrons can be modeled using SADDE Mod 2. The only input required is the electron energy in $\mathrm{MeV}$ and the electron yield expressed as a fraction. SADDE Mod 2 adds the contribution of the electrons to the beta spectrum, $\phi_{\beta}$, weighted by the yield of the electron. The total yield of the radionuclide is also adjusted to reflect the additional radionuclides.

Internal conversion and Auger electrons should only be modeled if the yield of the electron relative to the beta-particle yield is greater than 0.005 . In addition, electrons with energies less than $65 \mathrm{keV}$ can be ignored unless skin dose calculations at depths less than $7 \mathrm{mg} / \mathrm{cm}^{2}$ are needed. 


\section{Special Topics for Accurate Use of VARSKIN Mod 2}

\subsection{Infinite Sources}

VARSKIN Mod 2 is designed to be very flexible while maintaining the required accuracy. However, VARSKIN Mod 2 can be misused, particularly in modeling infinitely large sources (sources with all dimensions greater than the $\mathrm{X}_{99}$ distance for these radionuclides) and in volume-averaging doses for point sources. This section describes these two possible misuses of VARSKIN Mod 2 and how to avoid them, and provides a method to determine the maximum dose to $1 \mathrm{~cm}^{2}$ from multiple hot particles.

When modeling "infinite" or "semi-infinite" sources such as an enveloping cloud with VARSKIN Mod 2, the tendency is to choose very large dimensions for the source. This approach will result in the calculation of grossly inaccurate doses or zero doses because the integration routine becomes inaccurate. The correct method is to determine the maximum penetration distance (i.e., $1.8 \cdot \mathrm{X}_{90}$ distance, or $\mathrm{X}_{99}$ distance) and set the source dimensions accordingly.

The $\mathrm{X}_{99}$ distance can be found either by looking at the file BETADATA.DAT or by running a simple calculation for the radionuclide of interest. To find the radionuclide in BETADATA.DAT, look for the radionuclide name. The next line contains three values: the average beta energy, the $\mathrm{X}_{90}$ distance (in $\mathrm{cm}$ ), and the number of betas emitted per disintegration (yield). Multiplying the $\mathrm{X}_{90}$ distance by 1.8 gives the $\mathrm{X}_{99}$ distance. An alternative method of finding the $\mathrm{X}_{99}$ distance is to run a simple point source geometry problem with the radionuclide of interest. The $\mathrm{X}_{99}$ distance is printed on both the output screen and the output file as

$$
1.8 \cdot \mathrm{X}_{90} \text { distance : }
$$

When the $\mathrm{X}_{99}$ distance has been determined, the maximum source radius, $\mathrm{r}_{\max }$, for $\mathrm{x}$ and $y$-side lengths is determined using the equation:

$$
t_{\max }=t_{d o s}+\left(X_{g g} / \rho_{d}\right)
$$

where $\boldsymbol{r}_{\text {dos }}$ is the radius of the dose-averaging area (in $\mathrm{cm}$ ) and $\rho_{c}$ is the density of the covering material (in $\mathrm{g} / \mathrm{cm}^{3}$ ). If more than one covering material exists, use the density of the least dense material. Using the least-dense material will ensure that the area over which the dose averaged includes contributions from the entire source. If an air gap is used, use the density of air $\left(0.001293 \mathrm{~g} / \mathrm{cm}^{3}\right)$. If no cover material is used, use the density of tissue $\left(1 \mathrm{~g} / \mathrm{cm}^{3}\right)$. Remember to convert $r_{\max }$ from $\mathrm{cm}$ in Equation (5.1) to $\mu \mathrm{m}$ for VARSKIN Mod 2 input.

When modeling infinite sources, the use of three-dimensional slab geometry is recommended. When using slab geometry, the source thickness, $\Delta t_{\max }$, should be determined using the equation:

$$
\Delta t_{\max }=X_{9 g} / \rho_{s}
$$


VARSKIN Special Topics

\subsection{Volume-Averaged Doses for Point Sources}

\subsection{Maximum Dose to $1 \mathrm{~cm}^{2}$ from Multiple Hot Particles}

where $\rho_{\mathrm{s}}$ is the source density in $\mathrm{g} / \mathrm{cm}^{3}$. Again, remember to convert $\Delta \mathrm{t}_{\max }$ from $\mathrm{cm}$ in Equation (5.2) to $\mu \mathrm{m}$ for VARSKIN Mod 2 input.

Point sources have very steep dose gradients at the interface between the source and skin. Attempting to use the volume-averaged dose model in VARSKIN Mod 2 for point sources in direct contact with the skin will produce erroneous results because the cubic spline function does not accurately describe such steep gradients. For the same reason, modeling a point source as a two-dimensional disk with a very small radius will not alleviate the problem. Note that this problem occurs only when the point source is in direct contact with the skin.

The only accurate method for calculating volume-averaged doses for point sources in direct contact with skin is to insert a cover material with a thickness of $1 \mu \mathrm{m}$ and a density of $1 \mathrm{~g} / \mathrm{cm}^{3}$ between the source and skin. At this depth, the dose gradient is not nearly so steep, and the cubic spline function describes the depth dose profile accurately. When the results of the volume-averaged dose are reported, the skin depth should by increased by $0.1 \mathrm{mg} / \mathrm{cm}^{2}$ to account for the extra covering material.

Determining the maximum dose to $1 \mathrm{~cm}^{2}$ for multiple hot particles requires numerous calculations and cannot be performed quickly. The calculations also require elements that are not available in VARSKIN Mod 2 but that can be accomplished with some graph paper and a calculator.

Before attempting to run the offset particle model (Section 3.5), determine the dose directly beneath each of the hot particles. The output screen for each of the calculations will display the radius of the irradiated area if the dose-averaging area is large enough. If not, entering a larger area will eventually provide the radius of the irradiated area. By comparing these areas for each source, it may be possible to eliminate one or more of the particles because there are no overlapping fields associated with them. For particles with overlapping fields, the doses and their relative positions should then be plotted on a sheet of graph paper, leaving plenty of room between the sources for results from additional calculations.

Next, calculations using the offset particle model should be performed for locations midway between any two particles. For more than two sources that are not in a straight line, a central location should be chosen and the dose at this point should be calculated using the offset particle model. Thus, for three particles in a triangular formation, a total of four calculations should be performed, choosing the value of the offset to be one-half of the distance between any two sources, with one additional dose calculation performed in the center of the triangle.

After these calculations have been performed, it is left to the user's discretion to determine the most probable area of highest dose based on the distribution of dose on the graph paper. After determining this area, a final calculation for each particle using the offset particle model can be performed. An accuracy of greater than $20 \%$ should not be sought since the error associated with determining the relative positions of the particles on skin or protective clothing will usually exceed $20 \%$. 


\subsection{Short-Lived Radionuclides}

Care must be exercised when calculating skin doses from radionuclides that have short half lives relative to the exposure time. VARSKIN Mod 2 calculates the total beta and gamma doses assuming that no significant decay occurs during the exposure time. For short-lived nuclides, such as ${ }^{234 \mathrm{~m}} \mathrm{~Pa}\left(\mathrm{~T}_{1 / 2}=1.18 \mathrm{~min}\right)$, the source strength will decrease appreciably during most exposure times.

The method used to correct for a decaying source term is to multiply the calculated beta and/or gamma dose rate by a decay factor. That is, the corrected dose, $D_{\text {corr }}$, received during the exposure time, $\mathrm{t}_{\text {exp }}$, is given by

$$
D_{\text {corr }}=\frac{\dot{D}_{\text {init }} T_{1 / 2}}{.693}\left[1-\exp \left(-\frac{.693 t_{\text {exp }}}{T_{1 / 2}}\right)\right]
$$

where $D_{\text {init }}$ is initial dose rate in $\mathrm{rad} / \mathrm{hr}$ and $T_{1 / 2}$ is the half-life of the radionuclide in hours. 


\section{References}


Section 6

Traub, R. J., Reece, W. D., Scherpelz, R. I., and Sigalla, L. A. Dose Calculation for Contamination of the Skin Using the Computer Code VARSKIN. NUREG/CR-4418, U.S. Nuclear Regulatory Commission, Washington, D.C. (1987).

Unger, L. M., and Trubey, D. K. Specific Gamma-Ray Dose Constants for Nuclides Important to Dosimetry and Radiological Assessment, ORNL/RSIC-45/R1, Oak Ridge National Laboratory, Oak Ridge, Tennessee (1982).

Wilson, W. E., and W. D. Reece. Calculation of Percentile Distance Ratios and Sealed Absorbed Dose Distributions for 0.05-30 keV Primary Electrons. PNL-7848, Pacific Northwest Laboratory, Richland, Washington (1991). 
APPENDIX A

\section{BETA-DECAY RADIONUCLIDE DATABASE}




\section{APPENDIX A}

\section{BETA-DECAY RADIONUCLIDE DATABASE}

This appendix is designed to be used to determine input to the code SADDE Mod 2 (Reece, Miller, and Durham 1989), which calculates the beta spectrum for a specific radionuclide given the number of decay paths and the maximum energy, yield, and forbiddenness of each decay path. The output from SADDE Mod 2 is used as input for the code VARSKIN Mod 2.

The Beta-Decay Radionuclide Database contains the type, yield, and forbiddenness of each decay path for every beta-emitting radionuclide with a half-life greater than 8 hours. The Beta-Decay Radionuclide Database was generated by RADLST (Burrows 1988) using the Evaluated Nuclear Structure Data Files (ENSDF) obtained from the National Nuclear Data Center at Brookhaven National Laboratory (Upton, NY). The ENSDF format allows the coding of nuclear structure and decay data and associated textual documentation in a form which may be used by computers. The RADLST code is designed to calculate the type, yield, and energy of nuclear and atomic radiations associated with the radioactive decay of nuclei. It uses the ENSDF database as primary input. This code was used to calculate yields and maximum beta energies associated with a radionuclide that undergoes beta decay. The computer code LOGFT was used to determine the forbiddenness of each decay path.

The computer code SADDE Mod 2 calculates the scaled absorbed dose distribution for any beta-emitting radioisotope. As described in section 4, SADDE Mod 2 first generates the beta spectrum of the radioisotope. Beta spectra are classified according to the change in momentum and parity between parent and daughter nuclei. These classifications, as well as the assigned forbiddenness for each classification, are summarized in Table A.1. The beta transitions in ENSDF are classified either on the basis of the change in momentum and parity as shown in Table A.1 (if the dats is known) or on the basis of the systematics of Raman and Gove (1973) as summarized in Table A.2 if momentum and parity data are not known. The levels of forbiddenness are based on those assigned in ICRP-38 (1983). The values in the database (beginning on page A.3) are the maximum energy, yield, and forbiddenness.

\section{REFERENCES}

Burrows, T. W. 1988. The Program RADLST. Information Analysis Center Report, BNL-NCS-52142, Brookhaven National Laboratory, Upton, New York.

International Commission on Radiological Protection (ICRP). Radionuclide Transformations. ICRP

Publication 38, Pergamon Press, New York, 1983.

Raman, S., and N. G. Gove. 1973. "Rules for Spin and Parity Assignments Based on Log st Values." Phys. Rev. C7:1995.

Reece, W. D., S. D. Miller, and J. S. Durham. 1989. SADDE (Scaled Absorbed Dose Distribution Evaluator). NUREG/CR-5276, PNL-6761, U.S. Nuclear Regulatory Commission, Washington, D.C. 
Appendix A

Table A.1. Determination of Forbiddenness of Beta Spectra Based on Momentum and Parity Change

\begin{tabular}{lccc}
\hline \multicolumn{1}{c}{ Classification } & Momentum Change & Parity Change & Forbiddenness \\
\hline Superallowed $(\Delta \mathrm{T}=0)$ & $0^{+} \rightarrow 0^{+}$ & No & 0 \\
Isospin forbidden $(\Delta \mathrm{T}=0)$ & $0^{+} \rightarrow 0^{+}$ & No & 0 \\
Allowed & $0, \pm 1\left(\right.$ not 0 to $\left.0^{+}\right)$ & No & 0 \\
1st-forbidden nonunique & $0, \pm 1$ & Yes & 0 \\
1st-forbidden unique & \pm 2 & Yes & 1 \\
2nd-forbidden nonunique & \pm 2 & No & 1 \\
2nd-forbidden unique & \pm 3 & No & 2 \\
3rd-forbidden nonunique & \pm 3 & Yes & 2 \\
3rd-forbidden unique & \pm 4 & Yes & 3 \\
\hline
\end{tabular}

Table A.2. Determination of Forbiddenness Based on Log ( $\mathrm{ft}$ )

\begin{tabular}{lll}
\hline \multicolumn{1}{c}{ Classification } & \multicolumn{1}{c}{ LOGFT Ranges } & Forbiddenness \\
\hline Superallowed & $3.48<\log (\mathrm{ft})<3.5$ & 0 \\
Allowed & $3.6<\log (\mathrm{ft})<$ & 0 \\
& $5.9(\mathrm{a})$ & \\
Isospin forbidden $(\Delta \mathrm{T}=1)$ & $\log (\mathrm{ft})<8.5$ & 0 \\
Allowed & & 0 \\
1st-forbidden nonunique & & \\
Isospin forbidden $(\Delta \mathrm{T}=1)$ & $\log (\mathrm{ft})<11.0$ & 0 \\
Allowed & & 0 \\
1st-forbidden nonunique & & 1 \\
1st-forbidden unique & & \\
Isospin forbidden $(\Delta \mathrm{T}=1)$ & $\log (\mathrm{ft})<12.8$ & 0 \\
Allowed & & 0 \\
1st-forbidden nonunique & & 1 \\
1st-forbidden unique & & 1 \\
2nd-forbidden nonunique & & 1 \\
1st-forbidden unique & $\log (\mathrm{ft})>8.5$ & \\
\hline
\end{tabular}

(a) For the mass region around $\mathrm{Z}=82$, the upper limit should be lowered to 5.1. 


\section{BETA-DECAY RADIONUCLIDE DATABASE}

Note: The format used in this table is as follows:

Radionuclide Name (half life)

$\mathrm{E}_{\max }(\mathrm{MeV}) \quad$ Yield Forbiddenness

\begin{tabular}{|c|c|c|c|c|c|}
\hline \multicolumn{3}{|c|}{ 226AC B- DECAY } & \multicolumn{3}{|c|}{ 110AG B-DECAY (249.76 D) } \\
\hline 1.1170 & 0.24000 & 0 & 0.5304 & 0.30560 & 0 \\
\hline 1.0450 & 0.10000 & 0 & 0.4707 & 0.00080 & 0 \\
\hline \multirow[t]{2}{*}{0.8870} & 0.49000 & 0 & 0.1336 & 0.00410 & 0 \\
\hline & & & 0.0836 & 0.67100 & 0 \\
\hline \multicolumn{6}{|c|}{ 227AC B-DECAY } \\
\hline 0.0436 & 0.55000 & 0 & \multicolumn{3}{|c|}{ 111AG B-DECAY } \\
\hline 0.0343 & 0.35000 & 0 & 1.0370 & 0.92000 & 0 \\
\hline \multirow[t]{2}{*}{0.0191} & 0.10000 & 0 & 0.7916 & 0.01000 & 1 \\
\hline & & & 0.6949 & 0.07100 & 0 \\
\hline \multicolumn{6}{|c|}{ 228AC B- DECAY } \\
\hline 2.0840 & 0.10000 & 0 & \multicolumn{3}{|c|}{ 28AL B-DECAY } \\
\hline 1.9550 & 0.01900 & 0 & \multirow[t]{2}{*}{2.8642} & \multirow[t]{2}{*}{0.99990} & 0 \\
\hline 1.8140 & 0.00540 & 1 & & & \\
\hline 1.7460 & 0.11600 & 0 & \multicolumn{3}{|c|}{ 242AM B-DECAY $(16.02 \mathrm{H})$} \\
\hline 1.1730 & 0.31000 & 0 & 0.6625 & 0.37000 & 0 \\
\hline 1.126 & 0.00300 & 0 & \multirow[t]{2}{*}{0.6204} & \multirow[t]{2}{*}{0.46000} & 0 \\
\hline 1.1190 & 0.03000 & 0 & & & \\
\hline 1.0190 & 0.05820 & 0 & \multicolumn{3}{|c|}{ 244AM B-DECAY $(10.1 \mathrm{H})$} \\
\hline 0.9890 & 0.05600 & 0 & \multirow[t]{2}{*}{0.3871} & \multirow[t]{2}{*}{1.00000} & 0 \\
\hline 0.9740 & 0.03540 & 0 & & & \\
\hline 0.9150 & 0.00800 & 0 & \multicolumn{3}{|c|}{ 244AM B- DECAY (26 M) } \\
\hline 0.7100 & 0.00900 & 0 & 1.5150 & 0.65000 & 0 \\
\hline 0.6110 & 0.08100 & 0 & \multirow[t]{2}{*}{1.4720} & \multirow{2}{*}{0.30000} & 0 \\
\hline 0.6030 & 0.00180 & 0 & & & \\
\hline 0.5040 & 0.01150 & 0 & \multicolumn{3}{|c|}{ 246AM B- DECAY (25 M) } \\
\hline 0.4990 & 0.00780 & 0 & 2.3350 & 0.07000 & $\mathbf{0}$ \\
\hline 0.4960 & 0.04180 & 0 & 1.5360 & 0.16100 & 0 \\
\hline 0.4590 & 0.01180 & 0 & 1.5020 & 0.06900 & $\mathbf{0}$ \\
\hline 0.4540 & 0.02600 & 0 & 1.2990 & 0.37400 & 0 \\
\hline 0.4180 & 0.01570 & 0 & 1.2730 & 0.14700 & 0 \\
\hline \multicolumn{3}{|c|}{ 108AG B- DECAY (2.37 M) } & \multicolumn{3}{|c|}{ 246AM B- DECAY (39 M) } \\
\hline 1.6550 & 0.95400 & 0 & 1.1990 & 1.00000 & 0 \\
\hline \multirow[t]{2}{*}{1.0220} & 0.01760 & 0 & & & \\
\hline & & & \multicolumn{2}{|c|}{ 39AR B-DECAY } & \\
\hline \multicolumn{3}{|c|}{ 110AG B- DECAY (24.6 S) } & 0.5650 & 1.00000 & 1 \\
\hline 2.8928 & 0.95200 & 0 & & & \\
\hline 2.2353 & 0.04420 & 0 & & & \\
\hline
\end{tabular}


Appendix A

41AR B- DECAY

$\begin{array}{lll}2.4923 & 0.00800 & 1 \\ 1.1986 & 0.99000 & 0 \\ 0.8153 & 0.00052 & 0\end{array}$

74AS B- DECAY (17.77 D)

1.3539

0.7191

0.0843

0.18600

0.15400

0.00040

76AS B-DECAY

2.9635

2.4044

1.8412

1.7473

1.1758

0.5344

0.3082

$\begin{array}{ll}0.51000 & 1 \\ 0.35200 & 0 \\ 0.00810 & 1 \\ 0.07500 & 0 \\ 0.01770 & 0 \\ 0.01690 & 0 \\ 0.01030 & 0\end{array}$

77AS B-DECAY $(38.83 \mathrm{H})$

0.6831

0.4441

0.4333

0.1624

0.97000

0.01600

0.00630

0.00630

198AU B-DECAY

0.9607

0.2848

0.98700

199AU B-DECAY

0.4526

0.2942

0.2444

0.14300

0.66400

0.19300

200AU B- DECAY (18.7 H) 0.6100

1.00000

200AU B- DECAY (48.4 M)

2.2600

1.8900

1.2300

0.6900

0.6900

0.6700

0.6300
0.79000

0.04700

0.00230

0.00640

0.00020

0.10900

0.03900
140BA B-DECAY

$\begin{array}{lll}1.0040 & 0.24000 & 1 \\ 0.9900 & 0.38000 & 0 \\ 0.8710 & 0.04400 & 1 \\ 0.5660 & 0.09300 & 0 \\ 0.4530 & 0.23800 & 0\end{array}$

10BE B- DECAY

0.5562

$1.00000 \quad 2$

\section{BI B-DECAY}

$\begin{array}{lll}1.1614 & 1.00000 & 0\end{array}$

\section{BI B-DECAY}

0.5840

$0.00280 \quad 0$

212BI B-DECAY

2.2460

1.5190

$0.55310 \quad 0$

0.7330

$0.04490 \quad 0$

0.6250

0.5670

0.4400

$0.01460 \quad 0$

$0.01890 \quad 0$

$0.00244 \quad 0$

$0.00650 \quad 0$

214BI B- DECAY

3.2740

1.8960

1.8590

1.7310

1.6130

1.5440

1.5100

1.4270

1.3840

1.2790

1.2630

1.2570

1.1550

1.1260

1.0810

1.0700

0.8260

0.7920
$0.19900 \quad 0$

$0.07180 \quad 0$

$0.00870 \quad 0$

$0.00640 \quad 0$

$0.17500 \quad 0$

$0.16900 \quad 0$

$0.08260 \quad 0$

$0.01590 \quad 0$

$0.01380 \quad 0$

$0.01660 \quad 0$

$0.02900 \quad 0$

$0.04140 \quad 0$

$0.00560 \quad 0$

$0.00830 \quad 0$

$0.05540 \quad 0$

$0.02740 \quad 0$

$0.01450 \quad 0$
$0.03050 \quad 0$ 


$\begin{array}{ccc}249 B K \text { B- DECAY }(320 \mathrm{D}) & \\ 0.1257 & 0.99000 & 0 \\ 0.0632 & 0.01000 & 1 \\ & & \\ 250 \mathrm{BK} B-\text { DECAY } & & \\ 1.7810 & 0.04800 & 1 \\ 1.7380 & 0.04800 & 0 \\ 0.7490 & 0.83400 & 0 \\ 0.7100 & 0.06210 & 0\end{array}$

82BR B-DECAY

$\begin{array}{lll}0.4441 & 0.98500 & 0 \\ 0.2644 & 0.01300 & 0\end{array}$

14C B- DECAY

$\begin{array}{lll}0.1565 & 1.00000 & 0\end{array}$

$\begin{array}{ccc}\text { 45CA } \beta \text { - DECAY } & & \\ 0.2569 & 1.00000 & 0 \\ & & \\ 49 \text { CA } \beta \text { - DECAY } & & \\ 2.891 & 0.00410 & 0 \\ 2.178 & 0.91500 & 0 \\ 1.7465 & 0.00180 & 0 \\ 1.1912 & 0.07000 & 0 \\ 0.7699 & 0.00660 & 0 \\ 0.5249 & 0.00210 & 0\end{array}$

47CA B-DECAY

$\begin{array}{lll}1.9880 & 0.19000 & 0 \\ 0.6910 & 0.81000 & 0\end{array}$

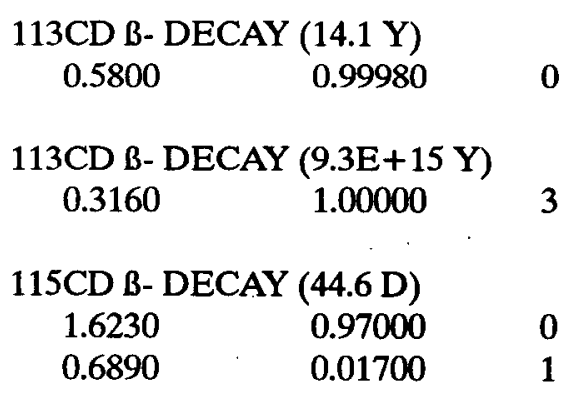

$\begin{array}{ccc}115 C D \text { B- DECAY } & (53.46 \mathrm{H}) & \\ 1.1060 & 0.62600 & 0 \\ 0.8450 & 0.01160 & 0 \\ 0.6130 & 0.03300 & 0 \\ 0.5780 & 0.33100 & 0\end{array}$

$\begin{array}{ccc}\text { 141CE B- DECAY } & & \\ 0.5813 & 0.29800 & 0 \\ 0.4359 & 0.70200 & 0\end{array}$

$\begin{array}{ccc}\text { 143CE } B \text { - DECAY } & & \\ 1.4042 & 0.35000 & 0 \\ 1.1110 & 0.48300 & 0 \\ 0.7397 & 0.13200 & 0 \\ 0.5238 & 0.01360 & 0\end{array}$

$\begin{array}{ccc}\text { 144CE B- DECAY } & & \\ 0.3182 & 0.76500 & 0 \\ 0.2381 & 0.03900 & 0 \\ 0.1847 & 0.19600 & 0\end{array}$

253CF B- DECAY

$\begin{array}{lll}0.2880 & 0.99700 & 0\end{array}$

36CL B- DECAY

$\begin{array}{lll}0.7092 & 0.98100 & 1\end{array}$

$\begin{array}{ccc}38 C L \text { B- DECAY } & & \\ 4.915 & 0.57600 & 1 \\ 2.748 & 0.10800 & 0 \\ 1.106 & 0.31600 & 0\end{array}$

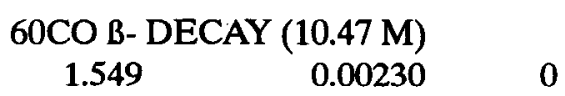

$\begin{array}{ccc}60 C O & B-D E C A Y & (5.2704 \\ 1.4911 & 0.00057 & 2\end{array}$

$\begin{array}{lll}0.3179 & 0.99925 & 0\end{array}$

61CO B-DECAY

$\begin{array}{lll}1.2547 & 0.95600 & 0 \\ 0.4129 & 0.04400 & 0\end{array}$

$\begin{array}{ccc}134 C S \beta-D E C A Y & & \\ 0.6570 & 0.7010 & 0 \\ 0.4152 & 0.0248 & 0 \\ 0.0886 & 0.2740 & 0\end{array}$


Appendix A

132CS B-DECAY

$\begin{array}{lll}0.8100 & 0.01570 & 0 \\ 0.2400 & 0.00370 & 0 \\ 0.1400 & 0.00060 & 0\end{array}$

135CS B- DECAY (2.3E+6 Y) 0.2050 $1.00000 \quad 0$

136CS B- DECAY (13.16 D) 0.6815

0.5176

0.4942

0.4079

0.3410

0.1744

0.13000

0.00200

0.04700

0.10500

0.70300

0.02040

137CS B-DECAY

$\begin{array}{lll}1.1732 & 0.05570 & 1 \\ 0.5115 & 0.94430 & 1\end{array}$

64CU B- DECAY

$\begin{array}{lll}0.5782 & 0.37100 & 0\end{array}$

67CU B- DECAY

$\begin{array}{lll}0.5760 & 0.20000 & 0 \\ 0.4830 & 0.23000 & 0 \\ 0.3910 & 0.56000 & 0 \\ 0.1820 & 0.01100 & 0\end{array}$

166DY B-DECAY

$\begin{array}{lll}0.4869 & 0.04900 & 0 \\ 0.4327 & 0.04900 & 1 \\ 0.4044 & 0.90200 & 0\end{array}$

169ER B- DECAY

$\begin{array}{lll}0.3520 & 0.55000 & 0 \\ 0.3436 & 0.45000 & 0\end{array}$

171ER $\beta$ - DECAY

1.4900

1.0651

0.8141

0.5770

0.4914
$0.02300 \quad 0$

$0.94000 \quad 0$

$0.00190 \quad 0$

$0.02190 \quad 0$

$0.00520 \quad 0$
172ER B- DECAY

0.8910

0.4840

$0.02800 \quad 1$

0.4160

$0.03100 \quad 0$

0.3560

$0.00940 \quad 0$

0.2810

$0.46700 \quad 0$

0.46480 . $\quad 0$

254ES B- DECAY (275.7 D)

0.1590

$1.00000 \quad 0$

254ES B- DECAY (39.3 H)

$\begin{array}{lll}1.1710 & 0.18000 & 0 \\ 1.1260 & 0.10000 & 0 \\ 1.0220 & 0.01000 & 0 \\ 0.4770 & 0.56000 & 0 \\ 0.4370 & 0.16000 & 0\end{array}$

150EU B-DECAY $(12.8 \mathrm{H})$

$1.0130 \quad 0.89000 \quad 0$

$\begin{array}{lll}0.3750 & 0.00020 & 1\end{array}$

152EU B-DECAY

$\begin{array}{lll}1.4778 & 0.08200 & 0 \\ 1.0667 & 0.00940 & 0 \\ 0.8915 & 0.00300 & 0 \\ 0.7129 & 0.00290 & 0 \\ 0.6989 & 0.13800 & 0 \\ 0.3881 & 0.02400 & 0 \\ 0.1787 & 0.01780 & 0\end{array}$

154EU B-DECAY

1.5979

$0.10000 \quad 0$

1.1534

0.9726

$0.00190 \quad 0$

0.8411

$0.00700 \quad 0$

$0.03500 \quad 0$

$0.16800 \quad 0$

$\begin{array}{lll}0.7173 & 0.00290 & 0\end{array}$

$\begin{array}{lll}0.7051 & 0.00670 & 0\end{array}$

$\begin{array}{lll}0.5713 & 0.36900 & 0\end{array}$

$\begin{array}{lll}0.4376 & 0.00280 & 0\end{array}$

$\begin{array}{lll}0.3517 & 0.01550 & 0\end{array}$

$\begin{array}{lll}0.3080 & 0.00800 & 0\end{array}$

$\begin{array}{lll}0.2493 & 0.28300 & 0\end{array}$ 


$\begin{array}{ccc}\text { 155EU B- DECAY } & & \\ 0.2527 & 0.13000 & 0 \\ 0.1927 & 0.08600 & 0 \\ 0.1662 & 0.28000 & 0 \\ 0.1474 & 0.50000 & 0 \\ 0.1347 & 0.00690 & 0 \\ 0.1066 & 0.00780 & 0\end{array}$

156EU B-DECAY

$\begin{array}{lll}2.4530 & 0.32000 & 0 \\ 1.4040 & 0.01300 & 0 \\ 1.2850 & 0.04100 & 0 \\ 1.2110 & 0.05300 & 0 \\ 1.0870 & 0.02100 & 0 \\ 0.5010 & 0.00900 & 0 \\ 0.4870 & 0.29000 & 0 \\ 0.4260 & 0.05700 & 0 \\ 0.2660 & 0.10300 & 0 \\ 0.2480 & 0.02200 & 0 \\ 0.1830 & 0.04200 & 0\end{array}$

157EU B- DECAY

$\begin{array}{lll}1.2990 & 0.48000 & 0 \\ 0.9290 & 0.15000 & 0 \\ 0.8880 & 0.22000 & 0 \\ 0.8480 & 0.03000 & 0 \\ 0.8380 & 0.04000 & 0 \\ 0.6800 & 0.04000 & 0 \\ 0.6760 & 0.03000 & 0 \\ 0.6120 & 0.02000 & 0\end{array}$

$\begin{array}{ccc}\text { 59FE } B \text { - DECAY } & & \\ 1.5650 & 0.00180 & 0 \\ 0.4660 & 0.53100 & 0 \\ 0.2730 & 0.45200 & 0 \\ 0.1310 & 0.01290 & 0\end{array}$

60FE B-DECAY

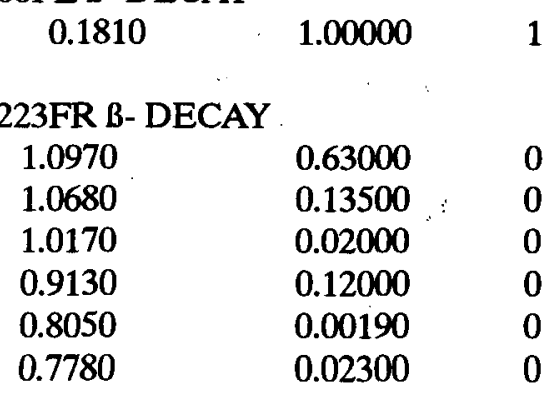

$\begin{array}{ccc}\text { 72GA B-DECAY } & & \\ 3.1584 & 0.10300 & 0 \\ 2.5284 & 0.08500 & 0 \\ 2.2641 & 0.00800 & 0 \\ 1.9274 & 0.02990 & 0 \\ 1.5900 & 0.00310 & 0 \\ 1.5285 & 0.00150 & 0 \\ 1.4776 & 0.08900 & 0 \\ 1.0489 & 0.01870 & 0 \\ 0.9568 & 0.27700 & 0 \\ 0.6674 & 0.21700 & 0 \\ 0.6506 & 0.15000 & 0 \\ 0.5530 & 0.00300 & 0 \\ 0.5371 & 0.00340 & 0 \\ 0.3143 & 0.00780 & 0\end{array}$

$\begin{array}{ccc}\text { 159GD B-DECAY } & & \\ 0.9747 & 0.62000 & 0 \\ 0.9167 & 0.26000 & 0 \\ 0.6265 & 0.00270 & 0 \\ 0.6112 & 0.11000 & 0\end{array}$

$\begin{array}{ccc}\begin{array}{cc}\text { 3H B-DECAY } \\ 0.0186\end{array} & 1.00000 & 0 \\ & & \\ \text { 181HF B-DECAY } & & \\ 0.5460 & 0.00500 & 1 \\ 0.4130 & 0.93000 & 0 \\ 0.4090 & 0.07000 & 0\end{array}$

$\begin{array}{ccc}182 \mathrm{HF} B-\text { DECAY } & (61.5 \mathrm{M}) & \\ 0.8940 & 0.08900 & 0 \\ 0.4310 & 0.37400 & 0 \\ 0.2100 & 0.01400 & 0\end{array}$

182HF B- DECAY (9E+6 Y)

$\begin{array}{lll}0.1040 & 1.00000 & 1\end{array}$

184HF B-DECAY

$\begin{array}{lll}1.1100 & 0.49000 & 0 \\ 0.8900 & 0.15000 & 0 \\ 0.7200 & 0.36000 & 0\end{array}$

203HG B-DECAY

$\begin{array}{lll}0.2126 & 1.00000 & 0\end{array}$


Appendix A

$\begin{array}{ccc}166 \mathrm{HO} \text { B- DECAY }(26.80 \mathrm{H}) & \\ 1.8539 & 0.50400 & 0 \\ 1.7733 & 0.48700 & 1 \\ 0.3940 & 0.00950 & 0 \\ & & \\ 166 \mathrm{HO} \text { B- DECAY } & (1200 \mathrm{Y}) & \\ 0.0729 & 0.74700 & 0 \\ 0.0324 & 0.17300 & 0 \\ & & \\ 129 \text { I B- DECAY } & & \\ 0.1540 & 1.00000 & 1 \\ & & \\ 130 \text { I B- DECAY } & & \\ 1.7810 & 0.01400 & 0 \\ 1.1780 & 0.01450 & 0 \\ 1.0420 & 0.47700 & 0 \\ 0.9040 & 0.00175 & 0 \\ 0.8140 & 0.02140 & 0 \\ 0.6240 & 0.46000 & 0 \\ 0.5590 & 0.00185 & 0 \\ 0.3780 & 0.00500 & 0 \\ 0.3570 & 0.00330 & 0 \\ 131 \text { I B- DECAY } & & \\ 0.8069 & 0.00420 & 1 \\ 0.6063 & 0.89400 & 0 \\ 0.3338 & 0.07360 & 0 \\ 0.3039 & 0.00620 & 0 \\ 0.2479 & 0.02130 & 0\end{array}$

$\begin{array}{ccc}\text { 133I B-DECAY } & & \\ 1.5300 & 0.01040 & 1 \\ 1.2300 & 0.83000 & 0 \\ 1.0200 & 0.01830 & 0 \\ 0.8800 & 0.04200 & 0 \\ 0.7100 & 0.00630 & 0 \\ 0.5200 & 0.03200 & 0 \\ 0.4600 & 0.03800 & 0 \\ 0.4100 & 0.00400 & 0 \\ 0.3700 & 0.01260 & 0\end{array}$

$\begin{array}{crc}\text { 115IN B-DECAY } & & \\ 0.8230 & 0.05000 & 0 \\ 0.3260 & 0.00047 & 0 \\ & & \\ 116 \text { IN B- DECAY } & (54.41 \mathrm{M}) & \\ 1.0120 & 0.52100 & 0 \\ 0.8740 & 0.33800 & 0 \\ 0.6020 & 0.10200 & 0 \\ 0.3570 & 0.02710 & 0\end{array}$

$\begin{array}{ccc}\text { 192IR B- DECAY } & & \\ 0.6720 & 0.48100 & 0 \\ 0.5360 & 0.41600 & 0 \\ 0.2560 & 0.05550 & 0\end{array}$

194IR B- DECAY (171 D)

$0.300 \quad 1.00$

0

132I B-DECAY

$\begin{array}{lll}2.1400 & 0.17600 & 0 \\ 1.6170 & 0.12700 & 0 \\ 1.4700 & 0.10200 & 0 \\ 1.4680 & 0.01900 & 0 \\ 1.4130 & 0.01700 & 0 \\ 1.2760 & 0.00910 & 0 \\ 1.1850 & 0.19000 & 0 \\ 1.1550 & 0.02110 & 0 \\ 0.9960 & 0.03790 & 0 \\ 0.9670 & 0.08100 & 0 \\ 0.9100 & 0.03600 & 0 \\ 0.8260 & 0.00370 & 0 \\ 0.7410 & 0.12800 & 0 \\ 0.7400 & 0.01810 & 0 \\ 0.6890 & 0.00880 & 0 \\ 0.5220 & 0.00340 & 0 \\ 0.4580 & 0.00380 & 0\end{array}$

194IR B-DECAY (19.15 H)

$\begin{array}{lll}2.2486 & 0.85400 & 0 \\ 1.9202 & 0.09200 & 0 \\ 1.6266 & 0.01350 & 0 \\ 1.3259 & 0.00300 & 1 \\ 0.9815 & 0.01760 & 0 \\ 0.7694 & 0.00640 & 0 \\ 0.7367 & 0.00560 & 0\end{array}$

40K B- DECAY $\begin{array}{lll}1.3121 & 0.8933 & 3\end{array}$

42K B- DECAY

$\begin{array}{lll}3.5251 & 0.81200 & 1 \\ 2.0002 & 0.18300 & 0 \\ 1.6876 & 0.00350 & 1\end{array}$


43K B- DECAY

$\begin{array}{lll}1.8170 & 0.01500 & 1 \\ 1.4440 & 0.03000 & 0 \\ 1.2240 & 0.03400 & 0 \\ 0.8270 & 0.89000 & 0 \\ 0.4220 & 0.03000 & 0\end{array}$

85KR B- DECAY (10.756 Y)

$\begin{array}{lll}0.6874 & 0.99563 & 1 \\ 0.1734 & 0.00434 & 0\end{array}$

$\begin{array}{ccc}87 \text { KR B- DECAY } & & \\ 3.8870 & 0.30500 & 0 \\ 3.4840 & 0.41000 & 0 \\ 3.0420 & 0.07300 & 1 \\ 2.4970 & 0.00119 & 0 \\ 2.3090 & 0.00120 & 0 \\ 2.1460 & 0.00620 & 0 \\ 1.4730 & 0.05500 & 0 \\ 1.3320 & 0.09400 & 0 \\ 1.0760 & 0.00600 & 0 \\ 0.9260 & 0.04400 & 0\end{array}$

138LA B-DECAY

0.33600

2

140LA B-DECAY

$\begin{array}{lll}2.1648 & 0.04800 & 0 \\ 1.6778 & 0.19200 & 0 \\ 1.4131 & 0.04930 & 0 \\ 1.4113 & 0.00360 & 1 \\ 1.3490 & 0.44000 & 0 \\ 1.2969 & 0.05450 & 0 \\ 1.2802 & 0.01070 & 0 \\ 1.2453 & 0.05680 & 0 \\ 1.2396 & 0.10900 & 0 \\ 1.2138 & 0.00630 & 1\end{array}$

176LU B- DECAY (3.6 E10 y)

$\begin{array}{lll}0.5970 & 0.99660 & 0 \\ 0.1959 & 0.00340 & 0\end{array}$

176LU B-DECAY $(3.68 \mathrm{H})$

$\begin{array}{ll}1.3170 & 0.39000\end{array}$

1.2286

$0.61000 \quad 0$

177LU B- DECAY (160.9 D)

0.1518

0.79000
177LU B-DECAY (6.71 D)

$\begin{array}{lll}0.4971 & 0.78600 & 0 \\ 0.3841 & 0.09100 & 0 \\ 0.1758 & 0.12200 & 0\end{array}$

179LU B-DECAY

$\begin{array}{lll}1.3500 & 0.87000 & 0 \\ 1.1400 & 0.10900 & 0 \\ 1.0100 & 0.02000 & 0\end{array}$

28MG B- DECAY

$\begin{array}{lll}0.8596 & 0.01400 & 0 \\ 0.4589 & 0.94000 & 0 \\ 0.2118 & 0.04600 & 0\end{array}$

56MN B- DECAY

$\begin{array}{lll}2.8489 & 0.56300 & 0 \\ 1.0382 & 0.27900 & 0 \\ 0.7358 & 0.14600 & 0 \\ 0.3260 & 0.01160 & 0\end{array}$

99MO B- DECAY

$\begin{array}{lll}1.2143 & 0.82400 & 0 \\ 0.8479 & 0.01140 & 0 \\ 0.4363 & 0.16500 & 0\end{array}$

24NA B-DECAY

1.3902

$0.99944 \quad 0$

94NB B-DECAY $(2.03 E+4 \mathrm{Y})$

$\begin{array}{lll}0.4716 & 0.98100 & 0\end{array}$

95NB B-DECAY (35.6 D)

$\begin{array}{lll}0.1597 & 0.99950 & 0\end{array}$

95NB B- DECAY $(86.6 \mathrm{H})$

$\begin{array}{lll}1.160 & 0.032 & 0 \\ 0.956 & 0.023 & 0 \\ 0.374 & 0.00068 & 0\end{array}$

96NB B-DECAY

$\begin{array}{lll}0.7490 & 0.96700 & 0 \\ 0.7460 & 0.02300 & 0 \\ 0.4320 & 0.00490 & 0 \\ 0.3120 & 0.00500 & 0\end{array}$

97NB B-DECAY

$\begin{array}{lll}1.2759 & 0.98340 & 0 \\ 0.9094 & 0.01080 & 0\end{array}$


Appendix A

$\begin{array}{ccc}98 N B \text { B - DECAY } & (51.3 \mathrm{M}) & \\ 3.2380 & 0.01400 & 0 \\ 3.1600 & 0.08000 & 0 \\ 2.6520 & 0.00800 & 1 \\ 2.5650 & 0.00850 & 0 \\ 2.4460 & 0.15900 & 0 \\ 2.2510 & 0.02060 & 1 \\ 2.1640 & 0.05200 & 1 \\ 2.0490 & 0.01140 & 0 \\ 1.9910 & 0.28600 & 0 \\ 1.9020 & 0.07700 & 0 \\ 1.6930 & 0.00760 & 0 \\ 1.6490 & 0.06500 & 0 \\ 1.6190 & 0.02310 & 0 \\ 1.4590 & 0.11000 & 0 \\ 1.3440 & 0.02850 & 0 \\ 1.2750 & 0.02900 & 0 \\ 1.2150 & 0.01750 & 0 \\ 1.1230 & 0.01240 & 0 \\ 0.9270 & 0.01110 & 0\end{array}$

239NP B-DECAY

$\begin{array}{lll}0.7142 & 0.04000 & 0 \\ 0.6648 & 0.02000 & 0 \\ 0.4366 & 0.53000 & 0 \\ 0.3919 & 0.07000 & 0 \\ 0.3304 & 0.33000 & 0 \\ 0.2102 & 0.01800 & 0\end{array}$

240NP B- DECAY (61.9 M) $\begin{array}{lll}1.9000 & 0.03200 & 0\end{array}$

240NP B- DECAY (7.22 M)

$\begin{array}{lll}2.1800 & 0.10000 & 0\end{array}$

$2.1370 \quad 0.42000 \quad 0$

$\begin{array}{lll}2.0380 & 0.01500 & 0\end{array}$

$\begin{array}{lll}1.5830 & 0.31000 & 0\end{array}$

$\begin{array}{lll}1.5310 & 0.00100 & 0\end{array}$

$\begin{array}{lll}1.3190 & 0.02500 & 0\end{array}$

$\begin{array}{lll}1.2800 & 0.03800 & 0\end{array}$

$\begin{array}{lll}1.2420 & 0.01400 & 0\end{array}$

$\begin{array}{lll}1.2210 & 0.01260 & 0\end{array}$

$\begin{array}{lll}0.7420 & 0.00360 & 0\end{array}$

$\begin{array}{lll}0.6920 & 0.00580 & 0\end{array}$

$\begin{array}{lll}0.6400 & 0.02300 & 0\end{array}$

$\begin{array}{ccc}1910 S B-D E C A Y & (15.4 D) & \\ 0.1414 & 1.00000 & 0\end{array}$

193OS B-DECAY

$\begin{array}{lll}1.1370 & 0.55000 & 0 \\ 1.0640 & 0.18000 & 0 \\ 0.9980 & 0.12500 & 0 \\ 0.9570 & 0.02000 & 0 \\ 0.7750 & 0.00770 & 0 \\ 0.6770 & 0.07900 & 0 \\ 0.5800 & 0.02400 & 0 \\ 0.5780 & 0.00740 & 0\end{array}$

$\begin{array}{ccc}\text { 194OS B- DECAY } & & \\ 0.0970 & 0.24000 & 0 \\ 0.0539 & 0.76000 & 0\end{array}$

32P B-DECAY

$\begin{array}{lll}1.7104 & 1.00000 & 0\end{array}$

33P B-DECAY

$\begin{array}{lll}0.2485 & 1.00000 & 0\end{array}$ 


$\begin{array}{ccc}\text { 230PA B-DECAY } & & \\ 0.5020 & 0.08200 & 0 \\ 0.1870 & 0.00180 & 0 \\ & & \\ 232 \text { PA B- DECAY } & & \\ 1.3370 & 0.00100 & 1 \\ 1.2890 & 0.00700 & 0 \\ 1.1800 & 0.00800 & 1 \\ 1.0150 & 0.00076 & 0 \\ 0.7740 & 0.01000 & 0 \\ 0.7080 & 0.00500 & 0 \\ 0.6460 & 0.00100 & 1 \\ 0.6020 & 0.00100 & 0 \\ 0.4700 & 0.01000 & 0 \\ 0.4260 & 0.00500 & 0 \\ 0.3200 & 0.73500 & 0 \\ 0.2860 & 0.25100 & 0\end{array}$

$\begin{array}{ccc}210 \mathrm{~PB} B-\text { DECAY } & & \\ 0.0630 & 0.20000 & 0 \\ 0.0165 & 0.80000 & 0 \\ 211 \mathrm{~PB} \text { B- DECAY } & & \\ 1.3790 & 0.91300 & 0 \\ 0.9740 & 0.01540 & 0 \\ 0.5470 & 0.06320 & 0 \\ & & \\ 212 \mathrm{~PB} \mathrm{B-DECAY} & & \\ 0.5730 & 0.11800 & 0 \\ 0.3340 & 0.83200 & 0 \\ 0.1580 & 0.05090 & 0\end{array}$

$\begin{array}{ccc}\text { 233PA B- DECAY } & & \\ 0.5721 & 0.08000 & 0 \\ 0.2599 & 0.17000 & 0 \\ 0.2316 & 0.40000 & 0 \\ 0.1735 & 0.16400 & 0 \\ 0.1563 & 0.27700 & 0\end{array}$

\section{PB B- DECAY}

$\begin{array}{lll}1.0320 & 0.09300 & 0 \\ 0.7370 & 0.40500 & 0 \\ 0.6800 & 0.46000 & 0 \\ 0.4980 & 0.01040 & 0 \\ 0.1930 & 0.02350 & 0\end{array}$

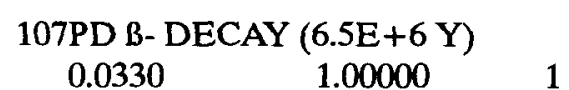

$\begin{array}{ccc}\text { 109PD B- DECAY } & & \\ 1.0279 & 0.99900 & 0\end{array}$

$\begin{array}{ccc}\text { 234PA B- DECAY }(1.17 \mathrm{M}) & \\ 2.2730 & 0.98400 & 0 \\ 1.4630 & 0.00620 & 0 \\ 1.2280 & 0.00740 & 0\end{array}$

234PA B-DECAY $(6.70 \mathrm{H})$

$\begin{array}{lll}1.2510 & 0.01100 & 0 \\ 1.2300 & 0.08000 & 0 \\ 1.1750 & 0.02600 & 0 \\ 1.1750 & 0.05500 & 0 \\ 1.1300 & 0.04000 & 0 \\ 1.1070 & 0.10000 & 0 \\ 0.9240 & 0.01400 & 0 \\ 0.7030 & 0.05000 & 0 \\ 0.6470 & 0.23000 & 0 \\ 0.5060 & 0.06800 & 0 \\ 0.4760 & 0.16000 & 0 \\ 0.4760 & 0.35000 & 0 \\ 0.4610 & 0.03300 & 0 \\ 0.4370 & 0.03100 & 0 \\ 0.4170 & 0.05700 & 0 \\ 0.3880 & 0.01400 & 0 \\ 0.3180 & 0.01800 & 0\end{array}$

\begin{tabular}{ccc}
$\begin{array}{cc}\text { 147PM B-DECAY } \\
0.2247\end{array}$ & 0.99994 & 0 \\
\multicolumn{3}{l}{ 148PM B-DECAY } \\
(41.29 D) & \\
1.0160 & 0.00800 & 0 \\
0.7040 & 0.22100 & 0 \\
0.5150 & 0.22700 & 0 \\
0.4160 & 0.50000 & 0
\end{tabular}

148PM B- DECAY (5.370 D)

$\begin{array}{lll}2.4730 & 0.55000 & 0 \\ 1.9230 & 0.09400 & 0 \\ 1.0490 & 0.00240 & 1 \\ 1.0080 & 0.33300 & 0\end{array}$

149PM B-DECAY

$\begin{array}{lll}1.0730 & 0.65100 & 0 \\ 1.0500 & 0.32600 & 0 \\ 0.7870 & 0.02300 & 0\end{array}$


Appendix A

$\begin{array}{ccc}1151 \text { PM B-DECAY } & \\ 1.1870 & 0.10000 & 0 \\ 1.1820 & 0.10000 & 0 \\ 1.1210 & 0.01300 & 0 \\ 1.1170 & 0.02700 & 0 \\ 1.0820 & 0.03000 & 0 \\ 1.0190 & 0.07900 & 0 \\ 1.0190 & 0.01400 & 0 \\ 0.9780 & 0.02300 & 0 \\ 0.8800 & 0.01700 & 0 \\ 0.8630 & 0.03300 & 0 \\ 0.8420 & 0.42000 & 0 \\ 0.7910 & 0.01900 & 0 \\ 0.7410 & 0.07100 & 0 \\ 0.6660 & 0.00770 & 0 \\ 0.4460 & 0.03100 & 0 \\ 0.4130 & 0.01140 & 0 \\ 0.3640 & 0.06100 & 0 \\ 0.2340 & 0.01000 & 0\end{array}$

200PT B- DECAY

$\begin{array}{lll}0.6900 & 0.19000 & 0 \\ 0.6300 & 0.01000 & 1 \\ 0.6100 & 0.46000 & 0 \\ 0.5900 & 0.08200 & 0 \\ 0.5200 & 0.00480 & 0 \\ 0.4500 & 0.12900 & 0 \\ 0.4500 & 0.00300 & 0 \\ 0.3900 & 0.09500 & 0 \\ 0.3000 & 0.06000 & 0 \\ 0.2200 & 0.00480 & 0\end{array}$

241PU B- DECAY

$\begin{array}{lll}0.0208 & 1.00000 & 0\end{array}$

243PU B-DECAY

$\begin{array}{lll}0.5830 & 0.60000 & 0 \\ 0.5400 & 0.08500 & 0 \\ 0.4990 & 0.21000 & 0 \\ 0.4860 & 0.01700 & 0 \\ 0.4730 & 0.05000 & 0 \\ 0.4390 & 0.03500 & 0 \\ 0.1170 & 0.01200 & 0\end{array}$

143PR B-DECAY $\begin{array}{lll}0.9345 & 1.00000 & 0\end{array}$

144PR B- DECAY (17.28 M)

$\begin{array}{lll}2.9960 & 0.97900 & 0 \\ 2.2990 & 0.01040 & 1 \\ 0.8100 & 0.01050 & 0\end{array}$

246PU B-DECAY

$\begin{array}{lll}0.3740 & 0.19000 & 1 \\ 0.3300 & 0.26200 & 0 \\ 0.1500 & 0.71000 & 0 \\ 0.0750 & 0.00836 & 0\end{array}$

225RA B-DECAY

$\begin{array}{lll}0.3710 & 0.30500 & 0 \\ 0.3310 & 0.69500 & 0\end{array}$

$\begin{array}{ccc}\text { 144PR B-DECAY } & (7.2 \mathrm{M}) & \\ 2.3590 & 1.00000 & 0\end{array}$

$\begin{array}{ccc}\text { 145PR B- DECAY } & & \\ 1.8050 & 0.97000 & 0 \\ 1.7330 & 0.00330 & 0 \\ 1.0570 & 0.01000 & 0 \\ 0.7540 & 0.00400 & 0\end{array}$

197PT B-DECAY (18.3 H)

$\begin{array}{lll}0.7190 & 0.11000 & 0 \\ 0.6417 & 0.81000 & 0 \\ 0.4502 & 0.08200 & 0\end{array}$

197PT B- DECAY (95.41 M) 0.7096

\section{RA B- DECAY

$\begin{array}{lll}0.0390 & 1.00000 & 0\end{array}$

86RB B- DECAY

$\begin{array}{lll}1.7743 & 0.9136 & 1 \\ 0.6973 & 0.0864 & 0\end{array}$

$\begin{array}{ccc}\text { 186RE B-DECAY } & & \text { ' } \\ 1.0745 & 0.79800 & 0 \\ 0.9373 & 0.20200 & 0\end{array}$

187RE B-DECAY

$\begin{array}{lll}0.0026 & 1.00000 & 1\end{array}$ 


$\begin{array}{ccc}\text { 188RE } \beta \text { - DECAY }(16.98 \mathrm{H}) & \\ 2.1202 & 0.70600 & 0 \\ 1.9652 & 0.26000 & 0 \\ 1.4872 & 0.01680 & 0 \\ 1.0338 & 0.00640 & 0 \\ 0.6577 & 0.00450 & 0 \\ & & \\ \text { 189RE B- DECAY } & & \\ 1.0080 & 0.64000 & 0 \\ 0.9770 & 0.04000 & 1 \\ 0.9720 & 0.03700 & 1 \\ 0.9380 & 0.02300 & 0 \\ 0.7910 & 0.13000 & 0 \\ 0.7890 & 0.07900 & 0 \\ 0.7320 & 0.05400 & 0 \\ 0.4080 & 0.01300 & 0\end{array}$

102RH B- DECAY (207 D)

$\begin{array}{lll}1.1390 & 0.18900 & 0 \\ 0.5820 & 0.01900 & 0\end{array}$

106RH B-DECAY (130 M)

$\begin{array}{lll}0.9220 & 0.85000 & 0\end{array}$

$\begin{array}{lll}0.7270 & 0.14500 & 0\end{array}$

106RH B- DECAY (29.8 S)

3.5400

3.0280

2.4120

2.4060

1.9780

1.5380

0.78600

0.08100

0.00640

0.10000

0.01770

0.00460

103RU B-DECAY

0.7660

0.4710

0.2290

0.1160

0.00870

0.00243

0.92200

0.06610

106RU B- DECAY

0.0394

$1.00000 \quad 0$

35S B-DECAY

0.1668

$1.00000 \quad 0$

122SB B-DECAY

1.9830

1.4190

0.7260

$\begin{array}{ll}0.26100 & 1 \\ 0.66800 & 0 \\ 0.04600 & 0\end{array}$

124SB B- DECAY (60.20 D)

$\begin{array}{lll}2.3023 & 0.23200 & 0 \\ 1.6564 & 0.02620 & 0 \\ 1.5795 & 0.04880 & 0 \\ 0.9471 & 0.02040 & 0 \\ 0.8657 & 0.03860 & 0 \\ 0.8134 & 0.00730 & 0 \\ 0.7225 & 0.00470 & 0 \\ 0.6113 & 0.51100 & 0 \\ 0.4217 & 0.00358 & 0 \\ 0.2113 & 0.08880 & 0\end{array}$

\section{SB B- DECAY}

0.6220

0.4457

0.3034

0.2416

0.1308

0.1246

0.0954

$\begin{array}{ll}0.13500 & 1 \\ 0.07140 & 0 \\ 0.40200 & 0 \\ 0.01620 & 0 \\ 0.18100 & 0 \\ 0.05790 & 0 \\ 0.13600 & 0\end{array}$

126SB B- DECAY (12.4 D)

1.8900

1.1700

$0.20000 \quad 1$

0.9000

0.8500

0.6800

0.6000

0.4900

0.4700

0.2100

$0.16000 \quad 0$

$0.04900 \quad 0$

$0.08100 \quad 0$

$0.04200 \quad 0$

$0.08400 \quad 0$

$0.05900 \quad 0$

$0.29000 \quad 0$

$0.02090 \quad 0$

127SB B- DECAY (3.85 D)

$\begin{array}{lll}1.4930 & 0.02000 & 1 \\ 1.2410 & 0.02100 & 0 \\ 1.1080 & 0.22500 & 0 \\ 0.9500 & 0.04400 & 0 \\ 0.8960 & 0.34300 & 0 \\ 0.7980 & 0.17200 & 0 \\ 0.7960 & 0.07870 & 0 \\ 0.6570 & 0.01100 & 0 \\ 0.5040 & 0.05200 & 0 \\ 0.4400 & 0.01590 & 0 \\ 0.4260 & 0.00900 & 0 \\ 0.2910 & 0.00740 & 0\end{array}$

46SC B- DECAY

$\begin{array}{lll}0.3567 & 0.99996 & 0\end{array}$ 
Appendix A

47SC B-DECAY

$\begin{array}{lll}0.6006 & 0.31700 & 0 \\ 0.4412 & 0.68300 & 0\end{array}$

48SC B-DECAY

$\begin{array}{lll}3.0100 & 0.00600 & 3 \\ 1.6980 & 0.00600 & 1 \\ 0.6610 & 0.90000 & 0 \\ 0.4850 & 0.09850 & 0\end{array}$

49SC B-DECAY

1.9940

$0.99940 \quad 0$

79SE B- DECAY

$$
\begin{array}{lll}
0.1490 & 1.00000 & 1
\end{array}
$$

31SI B-DECAY

$\begin{array}{lll}1.4908 & 0.99930 & 0 \\ 0.2247 & 0.00070 & 0\end{array}$

32SI B-DECAY

$$
\begin{array}{lll}
0.2130 & 1.00000 & 0
\end{array}
$$

151SM B-DECAY

$\begin{array}{lll}0.0763 & 0.99090 & 0\end{array}$

$\begin{array}{lll}0.0548 & 0.00910 & 0\end{array}$

153SM B- DECAY

$\begin{array}{lll}0.8098 & 0.20700 & 0\end{array}$

$\begin{array}{lll}0.7120 & 0.00570 & 0\end{array}$

$\begin{array}{lll}0.7070 & 0.43800 & 0\end{array}$

$\begin{array}{lll}0.6370 & 0.34700 & 0\end{array}$

156SM B-DECAY

$\begin{array}{lll}0.7140 & 0.00100 & 0 \\ 0.6910 & 0.52000 & 0 \\ 0.4230 & 0.45000 & 0\end{array}$

121SN B-DECAY (27.06 H)

$0.3866 \quad 1.00000$

121SN B- DECAY (55 Y)

0.3560

1.00000

123SN B- DECAY (129.2 D)

1.3970

0.3080

0.99370

0.00600 $\begin{array}{ccc}\text { 123SN B- DECAY } & (40.08 \mathrm{M}) & \\ 1.2610 & 0.99920 & 0\end{array}$

125SN B-DECAY (9.64 D)

2.3500

1.2610

$0.83000 \quad 1$

1.0010

$0.02820 \quad 0$

0.4600

$0.00331 \quad 1$

0.3670

$0.06180 \quad 0$

0.3480

$\begin{array}{ll}0.04020 & 0 \\ 0.02020 & 0\end{array}$

126SN B-DECAY

0.2500

$1.00000 \quad 0$

89SR B- DECAY

$\begin{array}{lll}1.4920 & 0.99990 & 1\end{array}$

90SR B- DECAY

$\begin{array}{lll}0.5460 & 1.00000 & 1\end{array}$

180TA B-DECAY

$\begin{array}{lll}0.7080 & 0.10500 & 0\end{array}$

0.6040

$0.03500 \quad 0$

182TA ß-DECAY

1.7135

1.4842

0.5922

0.5562

0.5244

0.4825

0.4398

0.3708

0.3261

$0.00058 \quad 0$

$0.00096 \quad 0$

$0.04000 \quad 0$

$0.00880 \quad 0$

$0.39700 \quad 0$

$0.02200 \quad 0$

0.20500 . 0

$0.00587 \quad 0$

0.2604

$0.01800, \quad 0$

183TA B-DECAY

$\begin{array}{lll}0.6584 & 0.02300 & 0\end{array}$

$\begin{array}{lll}0.6174 & 0.92000 & 0\end{array}$

$\begin{array}{lll}0.4752 & 0.00990 & 0\end{array}$

$0.4477 \quad 0.05000 \quad 0$ 


$\begin{array}{ccc}184 \mathrm{TA} \beta-\mathrm{DECAY} & & \\ 1.4400 & 0.00490 & 1 \\ 1.2800 & 0.00210 & 0 \\ 1.1900 & 0.00390 & 0 \\ 1.1700 & 0.86100 & 0 \\ 1.1200 & 0.15400 & 0 \\ 0.8400 & 0.00890 & 0 \\ 0.4800 & 0.00760 & 0 \\ 0.3700 & 0.00340 & 0\end{array}$

$\begin{array}{ccc}\text { 158TB B-DECAY } & & \\ 0.8400 & 0.16900 & 0 \\ 0.6220 & 0.01100 & 0\end{array}$

160TB B-DECAY

$\begin{array}{lll}1.7489 & 0.00340 & 0 \\ 0.8695 & 0.28800 & 0 \\ 0.7866 & 0.06300 & 0 \\ 0.6799 & 0.00200 & 0 \\ 0.5709 & 0.45600 & 0 \\ 0.5490 & 0.03440 & 0 \\ 0.4770 & 0.08540 & 0 \\ 0.4493 & 0.01010 & 0 \\ 0.4368 & 0.04390 & 0\end{array}$

161TB B- DECAY

0.5921

0.5664

0.5175

0.4603

$0.05000 \quad 0$

$0.05000 \quad 0$

$0.66000 \quad 0$

$0.26000 \quad 0$

98TC B- DECAY

0.3980

$1.00000 \quad 0$

99TC B- DECAY (2.111E+5 Y) 0.2936

0.99998

0

127TE B-DECAY $(9.35 \mathrm{H})$

0.6940

0.2760

0.98800

0.01190

127TE B- DECAY (109 D)

0.7250

0.02400
129TE B- DECAY (69.6 M)

$\begin{array}{lll}1.4700 & 0.88000 & 0 \\ 1.2190 & 0.00560 & 0 \\ 1.0100 & 0.09300 & 0 \\ 0.9380 & 0.00251 & 0 \\ 0.3860 & 0.00881 & 0\end{array}$

$\begin{array}{ccc}\text { 129TE } \beta \text { - DECAY } & (33.6 \mathrm{D}) & \\ 1.6040 & 0.33000 & 1 \\ 0.9080 & 0.03100 & 0 \\ 0.8740 & 0.00710 & 0\end{array}$

131TE B- DECAY (25.0 M)

$\begin{array}{lll}2.1000 & 0.59200 & 0 \\ 1.7570 & 0.00780 & 0 \\ 1.6480 & 0.21800 & 0 \\ 1.3730 & 0.01177 & 0 \\ 1.1520 & 0.02590 & 0 \\ 1.1030 & 0.09980 & 0 \\ 0.9520 & 0.00540 & 0 \\ 0.8230 & 0.01340 & 0 \\ 0.8060 & 0.01186 & 0 \\ 0.7490 & 0.01199 & 0\end{array}$

131TE B- DECAY $(30 \mathrm{H})$

$\begin{array}{lll}2.4320 & 0.03800 & 1 \\ 1.6590 & 0.00300 & 0 \\ 1.5800 & 0.00400 & 0 \\ 1.4260 & 0.00200 & 0 \\ 1.3730 & 0.00300 & 0 \\ 1.1170 & 0.00200 & 0 \\ 0.7860 & 0.02600 & 0 \\ 0.6350 & 0.00300 & 0 \\ 0.5450 & 0.01300 & 0 \\ 0.5330 & 0.16100 & 0 \\ 0.5080 & 0.02100 & 0 \\ 0.4520 & 0.37100 & 0 \\ 0.4310 & 0.05400 & 0 \\ 0.4210 & 0.02600 & 0 \\ 0.3690 & 0.00500 & 0 \\ 0.3180 & 0.02000 & 0 \\ 0.2640 & 0.01100 & 0 \\ 0.2620 & 0.00500 & 0 \\ 0.1910 & 0.00400 & 0 \\ 0.1620 & 0.00600 & 0\end{array}$

132TE B- DECAY

0.2150

$1.00000 \quad 0$ 
Appendix A

$\begin{array}{ccc}231 \text { TH B-DECAY } & & \\ 0.3310 & 0.00330 & 0 \\ 0.3119 & 0.00330 & 0 \\ 0.3054 & 0.35000 & 0 \\ 0.2882 & 0.37000 & 0 \\ 0.2873 & 0.12000 & 0 \\ 0.2154 & 0.01300 & 0 \\ 0.2061 & 0.12800 & 0 \\ 0.1423 & 0.02800 & 0\end{array}$

$\begin{array}{ccc}\text { 172TM B-DECAY } & & \\ 1.8700 & 0.29000 & 1 \\ 1.7910 & 0.36000 & 0 \\ 1.6100 & 0.00910 & 1 \\ 0.8270 & 0.00290 & 1 \\ 0.7520 & 0.00291 & 0 \\ 0.7150 & 0.00960 & 0 \\ 0.6980 & 0.06600 & 0 \\ 0.6710 & 0.00244 & 0 \\ 0.4040 & 0.10100 & 0 \\ 0.3930 & 0.01110 & 0 \\ 0.3210 & 0.02400 & 0 \\ 0.2620 & 0.10000 & 0 \\ 0.2070 & 0.01280 & 0\end{array}$

$\begin{array}{ccc}234 \text { TH B-DECAY } & & \\ 0.1960 & 0.72500 & 0 \\ 0.1040 & 0.18000 & 0 \\ 0.1030 & 0.07100 & 0 \\ 0.0830 & 0.02600 & 0\end{array}$

\section{TL B- DECAY

$\begin{array}{lll}0.7634 & 1.00000 & 1\end{array}$

206TL B-DECAY $\begin{array}{lll}1.5312 & 0.99900 & 0\end{array}$
207TL B-DECAY $\begin{array}{lll}1.4180 & 0.99760 & 0\end{array}$

208TL B- DECAY

$\begin{array}{lll}1.7960 & 0.48700 & 0 \\ 1.5190 & 0.21800 & 0 \\ 1.2860 & 0.24500 & 0 \\ 1.0740 & 0.00630 & 0 \\ 1.0330 & 0.03090 & 0\end{array}$

170TM B-DECAY $\begin{array}{lll}0.9681 & 0.76000 & 0\end{array}$ $\begin{array}{lll}0.8838 & 0.24000 & 0\end{array}$

$\begin{array}{ccc}\text { 171TM B- DECAY } & & \\ 0.0965 & 0.98000 & 0 \\ 0.0298 & 0.02000 & 0\end{array}$

237U B-DECAY

$\begin{array}{lll}0.2514 & 0.42000 & 0 \\ 0.2376 & 0.51000 & 0 \\ 0.1865 & 0.03400 & 0 \\ 0.1480 & 0.00800 & 0\end{array}$

240U ß- DECAY

$\begin{array}{lll}0.3600 & 0.29000 & 0 \\ 0.3160 & 0.61000 & 0\end{array}$

185W B-DECAY

$\begin{array}{lll}0.4328 & 0.99930 & 0\end{array}$

187W B-DECAY

$\begin{array}{lll}1.3124 & 0.29800 & 0 \\ 1.1782 & 0.00700 & 1 \\ 0.8006 & 0.00024 & 0 \\ 0.6940 & 0.04700 & 0 \\ 0.6869 & 0.03300 & 0 \\ 0.6266 & 0.54900 & 0 \\ 0.5395 & 0.04230 & 0 \\ 0.4856 & 0.00010 & 0 \\ 0.4478 & 0.00644 & 0 \\ 0.4329 & 0.00369 & 0\end{array}$


188W B-DECAY

0.3490

0.2850

0.0580

0.99000

0.00150

0.00830

133XE B- DECAY

0.3460

0.2660

0.99000

0.00810

$0.99984 \quad 1$

2.2792

91Y B-DECAY

1.5456

0.99700

1

93Y B- DECAY

2.8840

2.6170

1.9370

1.4590

1.4340

0.6990

$0.90400 \quad 1$

$0.04500 \quad 0$

$0.02500 \quad 0$

$0.00240 \quad 0$

$0.00330 \quad 0$

$0.01500 \quad 0$
175YB B-DECAY

0.4677

0.3539

0.2162

0.0714

$\begin{array}{ll}0.86500 & 0 \\ 0.03300 & 0 \\ 0.00800 & 1 \\ 0.10300 & 0\end{array}$

69ZN B-DECAY $(13.76 \mathrm{H})$

0.7700

1.00000

1

69ZN B- DECAY (56.4 M)

0.9060

$0.99999 \quad 0$

72ZN B- DECAY

$\begin{array}{lll}0.2960 & 0.85300 & 0 \\ 0.2490 & 0.14700 & 0\end{array}$

93ZR B-DECAY

0.0597

0.95000

1

95ZR B- DECAY

0.8870

0.3990

0.3660
$0.01110 \quad 1$

$0.44200 \quad 0$

\section{ZR B- DECAY}

$\begin{array}{lll}1.9151 & 0.88100 & 0 \\ 1.4075 & 0.04100 & 0 \\ 1.2249 & 0.00600 & 1 \\ 1.1100 & 0.00710 & 0 \\ 0.9079 & 0.00300 & 0 \\ 0.8941 & 0.01820 & 0 \\ 0.5526 & 0.05600 & 0\end{array}$




\section{APPENDIX B}

\section{INDEPENDENT VERIFICATION OF VARSKIN MOD 2 DOSE CALCULATIONS}




\section{APPENDIX B}

\section{INDEPENDENT VERIFICATION OF VARSKIN MOD 2 DOSE CALCULATIONS}

Verification of VARSKIN Mod 2 was performed by several independent users who agreed to test the program in a draft form and compare VARSKIN Mod 2 results to results obtained using other calculational methods. Most of the comments on the draft VARSKIN Mod 2 have been accomodated; the details of these comments will not be presented here. Several users compared the results obtained using VARSKIN Mod 2 to results obtained using independent methods, and those results are provided in this appendix.

\section{B.1 POINT SOURCE ON CLOTHING}

The model of Chabot et al. (1988) was used in this test. The types of protective clothing modelled were

- one layer of brown cotton coveralls, of thickness $0.37 \mathrm{~mm}$ and density $0.7 \mathrm{~g} \mathrm{~cm}^{-3}$ (1B)

- two layers of brown cotton coveralls, double the above thickness (2B)

- one layer of brown coveralls plus one layer of plastic suit material, the latter of thickness $0.4 \mathrm{~mm}$ and density $1.1 \mathrm{~g} \mathrm{~cm}^{-3}(\mathrm{~B}+\mathrm{P})$. For VARSKIN Mod 2 , the material densities were combined according to the instructions in this document.

The calculations were done for the isotopes ${ }^{60} \mathrm{Co},{ }^{137} \mathrm{Cs}$, and ${ }^{90} \mathrm{Sr} / \mathrm{Y}$, assuming a point source of $1 \mu \mathrm{Ci}$ of each and no self-absorption. Air gaps of 0.2 and $1.0 \mathrm{~cm}$ were used for the first two clothing types, and 1.0 and $5.0 \mathrm{~cm}$ for the third. It was assumed that there was no air gap between layers of protection clothing.

Gamma-ray doses were calculated using the analytical expression in Chabot et al. (1988), assuming no attenuation in clothing, no scatter, and $1 \mathrm{rem} R^{-1}$.'

The results of these calculations and the same calculations done with VARSKIN Mod 2 are shown in
Table B.1. In general, there is reasonable agreement between the two calculations for both the beta and gamma dose rate calculations. The largest differences occur for the case of ${ }^{137} \mathrm{Cs}$ on two layers of cotton coveralls.

\section{B.2 POINT SOURCE ON SKIN}

In this test, calculations were done for the case of a point source directly on the skin, i.e., no material and no air gap between the source and skin. For a $1-\mu \mathrm{Ci}$ hot particle on the skin, the beta dose rate to $1 \mathrm{~cm}^{2}$ at $7 \mathrm{mg} \mathrm{cm}^{-2}$ was calculated using both VARSKIN and VARSKIN Mod 2. For ${ }^{60} \mathrm{Co}$, a comparison of beta and gamma dose rates given by Chabot et al. (1988) to those predicted by VARSKIN Mod 2 was made. The results are shown in Table B.2. There is again reasonable agreement among the beta dose rates. The difference in the gamma dose calculation reflects the inclusion of charged particle equilibrium effects in VARSKIN $\operatorname{Mod} 2$.

\section{B.3 DISTRIBUTED CONTAMINATION ON SKIN}

The final set of calculations was for the case of distributed contamination on the skin. A concentration of $1 \mu \mathrm{Ci} \mathrm{cm}^{-2}$ over a circular area of $100 \mathrm{~cm}^{2}$ was assumed, and the beta dose rate to $1 \mathrm{~cm}^{2}$ of skin centered under the contamination was calculated. The calculations were done with both versions of VARSKIN, and the results compared with values published by Rohloff and Heinzelmann (1986) and by Kocher and Eckerman (1987). The results from VARSKIN Mod 1, VARSKIN Mod 2, and Kocher and Eckerman (1987), shown in Table B.3, agree quite well, while those from Rohloff and Heinzelmann (1986) are somewhat lower because they neglected backscatter from air. At the time of this test, the backscatter model had not been incorporated into VARSKIN Mod 2. 
Appendix B

Table B.1. Comparison of Chabot's Method and VARSKIN Mod 2 for 1- $\mu$ Ci Hot Particle Point Source on Clothing

\begin{tabular}{|c|c|c|c|c|c|c|c|c|}
\hline \multirow[b]{2}{*}{ Isotope } & \multirow[b]{2}{*}{$\begin{array}{l}\text { Air Gap } \\
\text { (cm) }\end{array}$} & \multirow[b]{2}{*}{ Clothing } & \multicolumn{3}{|c|}{ Beta Dose Rate (rem h ${ }^{-1}$ ) } & \multicolumn{3}{|c|}{ Gamma Dose Rate $\left(\mathrm{rem} \mathrm{h}^{-1}\right)$} \\
\hline & & & $\begin{array}{l}\text { Chabot } \\
\text { et al. }\end{array}$ & $\begin{array}{l}\text { VARSKIN } \\
\operatorname{Mod} 2\end{array}$ & $\begin{array}{l}\text { Diff. } \\
(\%)\end{array}$ & $\begin{array}{l}\text { Chabot } \\
\text { et al. }\end{array}$ & $\begin{array}{l}\text { VARSKIN } \\
\text { Mod } 2\end{array}$ & $\begin{array}{l}\text { Diff. } \\
\text { (\%) }\end{array}$ \\
\hline \multirow[t]{6}{*}{${ }^{60} \mathrm{Co}$} & 0.2 & 1B & 0.203 & 0.194 & -4 & 0.077 & 0.0725 & -6 \\
\hline & 1.0 & 1B & 0.080 & 0.078 & -2 & 0.011 & 0.0102 & -7 \\
\hline & 0.2 & 2B & 0.002 & $\mathbf{0}$ & - & 0.067 & 0.064 & -5 \\
\hline & 1.0 & 2B & 0.004 & $\mathbf{0}$ & - & 0.010 & 0.0096 & -4 \\
\hline & 1.0 & $\mathbf{B}+\mathbf{P}$ & 0 & $\mathbf{0}$ & - & 0.010 & 0.0095 & -5 \\
\hline & 5.0 & $\mathbf{B}+\mathbf{P}$ & $\mathbf{0}$ & $\mathbf{0}$ & - & 0.0005 & 0.0005 & $\mathbf{0}$ \\
\hline \multirow[t]{6}{*}{${ }^{137} \mathrm{Cs}$} & 0.2 & $1 B$ & 1.33 & 1.39 & +4 & 0.019 & 0.020 & +5 \\
\hline & 1.0 & 1B & 0.295 & 0.275 & -7 & 0.0027 & 0.0028 & +4 \\
\hline & 0.2 & $2 B$ & 0.357 & 0.474 & +32 & 0.0167 & 0.0175 & +5 \\
\hline & 1.0 & $2 B$ & 0.121 & 0.140 & +16 & 0.0025 & 0.0026 & +4 \\
\hline & 1.0 & $\mathbf{B}+\mathbf{P}$ & 0.067 & 0.075 & +12 & 0.0025 & 0.0026 & +4 \\
\hline & 5.0 & $\mathrm{~B}+\mathrm{P}$ & 0.0035 & 0.0037 & +6 & 0.0001 & 0.0001 & 0 \\
\hline \multirow[t]{6}{*}{${ }^{90} \mathrm{Sr} / \mathrm{Y}$} & 0.2 & $1 B$ & 3.534 & 3.68 & +4 & & & \\
\hline & 1.0 & $1 B$ & 0.596 & 0.587 & -2 & & & \\
\hline & 0.2 & $2 B$ & 2.245 & 2.38 & +6 & & & \\
\hline & 1.0 & $2 \mathrm{~B}$ & 0.409 & 0.438 & +7 & & & \\
\hline & 1.0 & $\mathrm{~B}+\mathrm{P}$ & 0.337 & 0.365 & +8 & & & \\
\hline & 5.0 & $\mathrm{~B}+\mathrm{P}$ & 0.018 & 0.0185 & +3 & & & \\
\hline
\end{tabular}




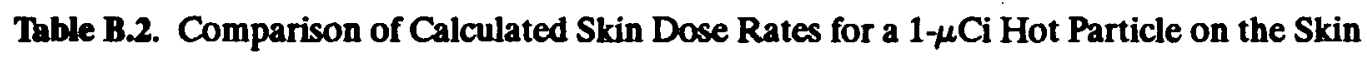

\begin{tabular}{clllll}
\hline & \multicolumn{3}{c}{ Beta Dose Rate $\left(\mathrm{rad} \mathrm{h}^{-1}\right)$} & \multicolumn{2}{c}{ Gamma Dose Rate $\left(\mathrm{rad} \mathrm{h}^{-1}\right)$} \\
\cline { 2 - 6 } Isotope & VARSKIN & VARSKIN Mod 2 & Chabot & VARSKIN Mod 2 & Chabot \\
\hline${ }^{60} \mathrm{Co}$ & 4.13 & 4.12 & 4.33 & 0.185 & 0.364 \\
${ }^{137} \mathrm{Cs}$ & 6.42 & 6.36 & - & 0.075 & - \\
${ }^{90} \mathrm{Sr} / \mathrm{Y}$ & 15.9 & 15.8 & - & - & - \\
\hline
\end{tabular}

Table B.3. Comparison of Calculated Beta Skin Doses for Distributed Contamination of $1 \mu \mathrm{Ci} \mathrm{cm}{ }^{-2}$ (over $100 \mathrm{~cm}^{2}$ ) on the Skin

\begin{tabular}{ccccc}
\hline & \multicolumn{4}{c}{ Beta Dose Rate (rad h $\left.{ }^{-1}\right)$} \\
\cline { 2 - 5 } Isotope & VARSKIN & VARSKIN Mod 2 & $\begin{array}{c}\text { Rohloff and } \\
\text { Heinzelmann (1986) }\end{array}$ & $\begin{array}{c}\text { Kocher and } \\
\text { Eckerman (1987) }\end{array}$ \\
\hline${ }^{60} \mathrm{Co}$ & 4.13 & 4.07 & 3.24 & 4.18 \\
${ }^{137} \mathrm{Cs}$ & 6.46 & 6.3 & - & 5.91 \\
${ }^{90} \mathrm{Sr} / \mathrm{Y}$ & 16.1 & 15.7 & 12.06 & 15.61 \\
\hline
\end{tabular}

D. Delacroix and J. P. Bourion of Centre D'Études Nucleaires de Saclay compared the results of calculations using VARSKIN Mod $2(V)$ and the work of the following:

- (D): Delacroix 1986

- (K): Kocher and Eckerman 1987

- (P): Piechowski et al. 1988.

These three papers give maximum dose rates (B e) from a source modelled as an "infinitely thick," uniformly. contaminated $\left(1 \mu \mathrm{Ci} / \mathrm{cm}^{2}\right)$ plane surface. Doses were calculated at different depths in the skin. The

VARSKIN Mod 2 input data were

- 2-D disk geometry

- 2-cm diameter

- $3.14 \mu \mathrm{Ci}$ activity.

Tables B.4 and B.5 show that the results are in good agreement. 
Appendix B

Table B.4. 70- $\mu \mathrm{m}$ Depth Dose Rates

\begin{tabular}{|c|c|c|c|c|c|c|}
\hline \multicolumn{2}{|c|}{$\begin{array}{l}\mathrm{rad} / \mathrm{h} \text { per } \\
\mu \mathrm{Ci} / \mathrm{cm}^{2}\end{array}$} & $\frac{{ }^{14} \mathrm{C}}{1.08}$ & $\frac{{ }^{32} \mathrm{P}}{8.94}$ & $\frac{{ }^{131} \mathrm{I}}{6.22}$ & $\frac{{ }^{90} \mathrm{Sr}}{6.67}$ & $\frac{{ }^{90} \mathrm{Y}}{9.04}$ \\
\hline $\mathbf{V}$ & $\begin{array}{l}\text { a } \\
\text { b } \\
\text { c } \\
\text { d } \\
\text { e }\end{array}$ & $\begin{array}{l}1.08 \\
1.08 \\
1.08 \\
1.07 \\
1.06\end{array}$ & $\begin{array}{l}8.94 \\
9.07 \\
9.09 \\
9.09 \\
9.09\end{array}$ & $\begin{array}{l}6.22 \\
6.21 \\
6.24 \\
6.23 \\
6.23\end{array}$ & $\begin{array}{l}6.67 \\
6.60 \\
6.65 \\
6.73 \\
6.72\end{array}$ & $\begin{array}{l}9.04 \\
8.17 \\
9.21 \\
9.21 \\
9.21\end{array}$ \\
\hline D & & 1.07 & 9.15 & 6.42 & 6.99 & 9.18 \\
\hline $\mathbf{K}$ & & 1.22 & 8.87 & 6.34 & 6.76 & 8.87 \\
\hline $\mathbf{P}$ & & 1.2 & 7.0 & 6.0 & 5.9 & 7.5 \\
\hline
\end{tabular}

a: beta dose rate averaged over $1 \mathrm{~cm}^{2}$

b: beta dose rate averaged over $0.1 \mathrm{~cm}^{2}$

c: beta dose rate averaged over $0.01 \mathrm{~cm}^{2}$

$\mathrm{d}$ : beta dose rate averaged over $0.001 \mathrm{~cm}^{2}$

e: beta dose rate averaged over $0.0001 \mathrm{~cm}^{2}$

Table B.5. Dose Rates Versus Depth for ${ }^{90} \mathrm{Y}$ Averaged Over $0.01 \mathrm{~cm}^{2}$

\begin{tabular}{ccccc}
\hline $\begin{array}{c}\mathrm{rad} / \mathrm{h} \text { per } \\
\mu \mathrm{Ci} / \mathrm{cm}^{2}\end{array}$ & $40 \mu \mathrm{m}$ & $70 \mu \mathrm{m}$ & $100 \mu \mathrm{m}$ & $400 \mu \mathrm{m}$ \\
\hline $\mathrm{V}$ & 10.6 & 9.21 & 8.38 & 5.18 \\
$\mathrm{D}$ & 10.46 & 9.18 & 8.37 & 5.24 \\
$\mathrm{~K}$ & 10.14 & 8.87 & - & 5.07 \\
\hline
\end{tabular}

\section{REFERENCES}

Chabot, G. E., K. W. Krable, and C. S. French. 1988. When Hot Particles are Not on the Skin. Radiation Protection Management 5(6):31-42.

Delacroix, D. 1986. Beta Particle and Electron Absorbed Dose Calculations for Skin Surface Contaminations (in French). DCES/SPR/SRI/86-656, Commissariat a l'Energie Atomique, Paris.
Kocher, D. C., and K. F. Eckerman. 1987. Electron Dose-Rate Conversion Factors for External Exposure of the Skin from Uniformly Deposited Activity on the Body Surface. Health Physics 53:135-141.

Piechowski et al. 1988. Dosimetry and Therapy of Skin Contaminations (in French). CEA-R-5441, Commissariat a l'Energie Atomique, Paris.

Rohloff, F., and M. Heinzelmann. 1986. Calculation of Dose Rates for Skin Contamination by Beta Radiation. Radiation Protection Dosimetry 14:279-287. 


\section{APPENDIX C}

\section{TEXT OF VARSKIN MOD 2 CODE}

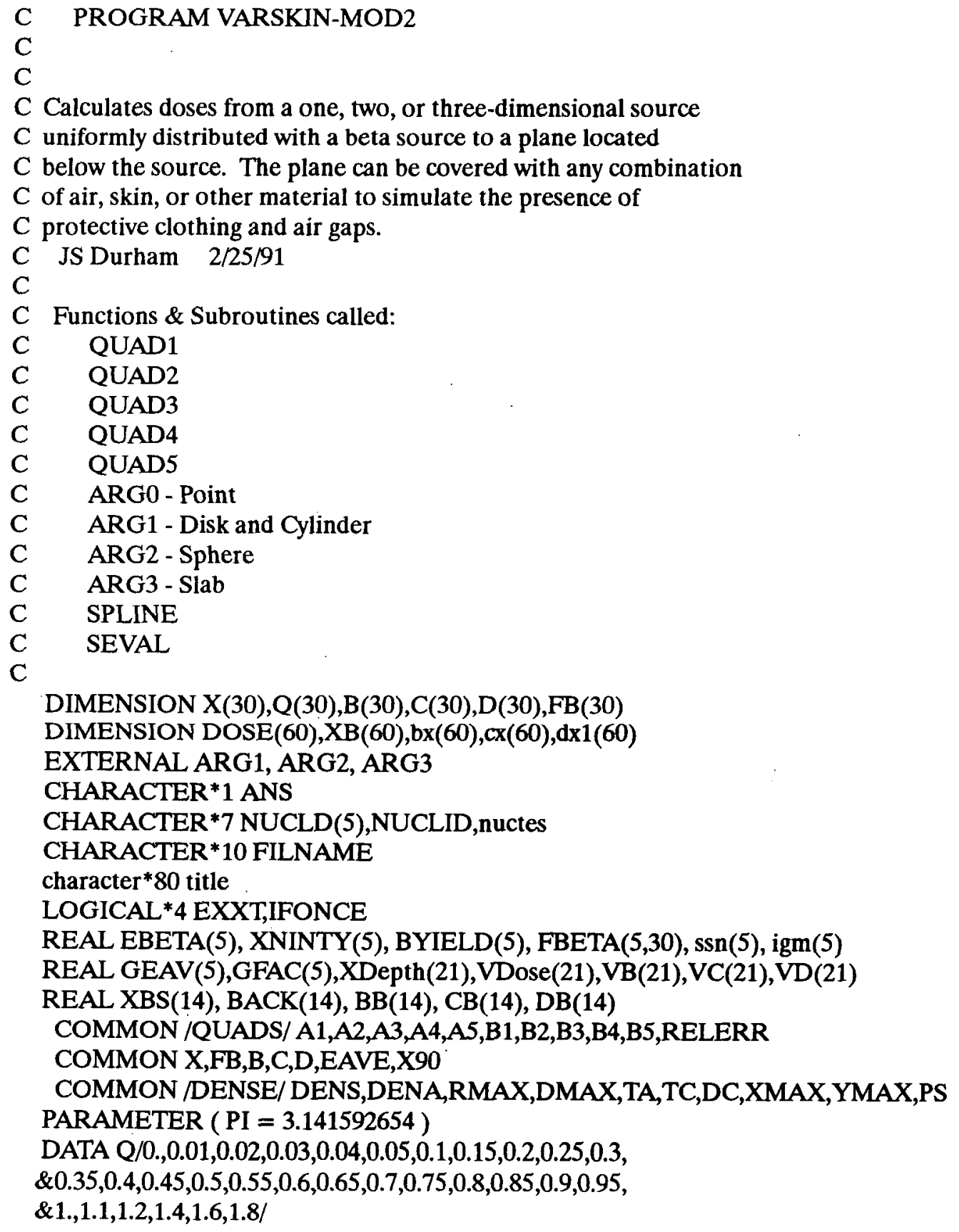


Appendix C

DATA xbs/2.67,4.77,9.00,13.71,18.71,24.14,29.57,35.29,

\& $\quad 41.00,46.86,75.71,105.71,165.71,224.29 /$

DATA BACK/1.0,1.13,1.22,1.27,1.30,1.32,1.34,1.35,1.36,1.36,

\& $1.34,1.33,1.31,1.28 /$

DATA ifonce/.true./

C

C THIS ROUTINE READS IN THE BERGER SCALED ABSORBED DOSE DISTRIBUTION, C SET UP THE INTEGRATION AND GENERATES THE OUTPUT

C.

DO $1 \mathrm{I}=1,30$

$$
\mathrm{X}(\mathrm{I})=\mathrm{Q}(\mathrm{I})
$$

1 CONTINUE

3 format( a1)

31 format( a )

filname $=$ 'VMOD2.OUT'

INQUIRE $($ FILE $=$ filname, EXIST $=$ EXXT)

IF (EXXT) THEN

ICR $=64$

FILNAME(1:5)='VMOD2'

FILNAME $(7: 10)=$ '.OUT'

$4 \quad \mathrm{ICR}=\mathrm{ICR}+1$

IF(ICR.GT.90)THEN

WRITE $\left({ }^{*},{ }^{*}\right)$ ' VM2OUTA-Z.OUT ALL EXIST'

WRITE $\left({ }^{*},{ }^{*}\right)$ ' PLEASE ERASE OR RENAME FILES AND RESTART THE PROGRAM' GO TO 800

ENDIF

FILNAME $(6: 6)=$ CHAR(ICR)

INQUIRE $($ FILE $=$ FILNAME, EXIST $=$ EXXT)

IF (EXXT) GO TO 4

$\mathrm{C}$

ENDIF

C Read Input Data

$\mathrm{C}$

OPEN (UNIT =13, FILE = 'OUTPUT.DAT',STATUS = 'OLD')

READ(13,5)rmax 1

$\operatorname{READ}(13,5) \times \max 1$

$\operatorname{READ}(13,5) \mathrm{ymax} 1$

$\operatorname{READ}(13,5)$ dens

READ(13,5)relerr

$\operatorname{READ}(13,5) \mathrm{dmax} 1$

$\operatorname{READ}(13,5) \mathrm{tc} 1$

$\operatorname{READ}(13,5) \mathrm{dc}$

$\operatorname{READ}(13,5)$ dth 1

$\operatorname{READ}(13,5)$ ta 1

$\operatorname{READ}(13,5)$ ttime

$\operatorname{READ}(13,5)$ earea

READ(13,51)igeo

$\operatorname{READ}(13,51)$ nnuc

$\operatorname{READ}(13,3)$ ans 
ivol $=0$

if(ans.eq.'y'.or.ans.eq.' 'Y')ivol $=1$

$\operatorname{READ}(13,51)$ isors

if(igeo.eq.0)isors $=0$

do $\mathrm{nn}=1$,nnuc

$\operatorname{READ}(13,3)$ ans

$\operatorname{igm}(\mathrm{nn})=0$

if(ans.eq.'y'.or.ans.eq.'Y')igm(nn) $=1$

$\operatorname{READ}(13,74)$ nuctes

backspace(13)

if(nuctes(4:4).eq.' ')then

$\operatorname{READ}(13,7)$ nucld(nn),ebeta(nn),xninty(nn),byield(nn),

1 (fbeta(nn,j),j=1,30)

elseif(nuctes(5:5).eq.' ')then

$\operatorname{READ}(13,71)$ nucld(nn), ebeta(nn),xninty(nn),byield(nn),

1 (fbeta(nn,j),j=1,30)

elseif(nuctes(6:6).eq.' ')then

$\operatorname{READ}(13,72)$ nucld(nn), ebeta(nn),xninty(nn),byield(nn),

1 (fbeta(nn,j),j=1,30)

elseif(nuctes(7:7).eq.' ')then

$\operatorname{READ}(13,73)$ nucld(nn), ebeta(nn),xninty(nn),byield(nn),

1 (fbeta(nn,j),j=1,30)

else

READ(13,74)nucld(nn), ebeta(nn),xninty(nn), byield(nn),

1 (fbeta(nn,j),j=1,30)

endif

$\operatorname{READ}(13,8) \operatorname{geav}(\mathrm{nn}), \mathrm{gfac}(\mathrm{nn})$

$\operatorname{READ}(13,5) \operatorname{ssn}(\mathrm{nn})$

enddo

5 format(f12.0)

51 format(i1)

7 FORMAT(a3,3X,3F9.4,/4X,10F8.4,/4X,10F8.4,/4X,10F8.4)

71 FORMAT(a4,3X,3F9.4,/4X,10F8.4,/4X,10F8.4,/4X,10F8.4)

72 FORMAT(a5,3X,3F9.4,/4X,10F8.4,/4X,10F8.4,/4X,10F8.4)

73 FORMAT(a6,3X,3F9.4,/4X,10F8.4,/4X,10F8.4,/4X,10F8.4)

74 FORMAT(a7,3X,3F9.4,/4X,10F8.4,/4X,10F8.4,/4X,10F8.4)

8 FORMAT(2F9.4)

C

C Initialize Variables

C

$$
\begin{aligned}
& \text { idos }=0 \\
& \text { IPAR }=0 \\
& \text { rmax }=\operatorname{rmax} 1 / 20000 . \\
& \text { dth }=d t h 1 / 10000 . \\
& \text { xmax }=x \max 1 / 20000 . \\
& \text { ymax }=y \max 1 / 20000 . \\
& \text { dmax }=\text { dmax } 1 / 1000 . \\
& \text { tc }=\text { tc } 1 / 10 . \\
& \text { ta }=\text { ta } 1 / 10 .
\end{aligned}
$$


Appendix C

dena $=.001293$

$\mathrm{npt}=26$

C

C Start the loop for multiple radionuclides

$\mathrm{C}$

do $10 \mathrm{jj}=1$,nnuc

NUCLID $=$ NUCLD $(j i)$

$\mathrm{EAVE}=\operatorname{EBETA}(\mathrm{jj})$

$\mathrm{X} 90=\mathrm{XNINTY}(\mathrm{jj})$

YIELD = BYIELD $(\mathrm{jj})$

geavg $=\operatorname{geav}(\mathrm{ji})$

gamfac $=\operatorname{gfac}(\mathrm{jj})$

$\mathrm{SS}=\operatorname{ssn}(\mathrm{jj})$

igam $=$ igm $(\mathrm{jj})$

DO 1150 IX $=1,30$

$1150 \quad \mathrm{FB}(\mathrm{IX})=\mathrm{FBETA}(\mathrm{jj}, \mathrm{IX})$

$\mathrm{C}$

C Spline fit for $\mathrm{X}$ vs chosen FB array--for later interpolations

$\mathrm{C}$

CALL SPLINE(30,X,FB,B,C,D)

darea $=1$.

bsfa $=1$.

bsf $1=1$.

ss $1=$ ss

dist $=1.8 * \mathrm{X} 90$

$\mathrm{C}$

C Determine if the gamma dose can be calculated

$\mathrm{C}$

$\operatorname{dimax}=2 .^{*} \max (x \max , y \max )$

if(igeo.ge.1.and.igeo.le.3.and.rmax.gt.0.1)igam $=0$

if(igeo.eq.4.and.dimax.gt.0.1)igam $=0$

if(ta.gt.5.)igam $=0$

C

if (ivol.eq.1)igam $=0$

C Spline fit for Backscatter model

C

If(igeo.eq.1.or.igeo.eq.0)then

$\mathrm{C}$

CALL SPLINE(14,xbs,back,Bb,Cb,Db)

C Calculate the Backscatter factor (used for igeo $=0,1$ only)

$\mathrm{C}$

if $\left(\mathrm{dmax}+\mathrm{tc}^{*} \mathrm{dc}+\mathrm{ta}^{*} \mathrm{dena}\right.$.gt.0.) then

$\mathrm{xbs} 1=\mathrm{X} 90 /\left(\mathrm{dmax}+\mathrm{tc}^{*} \mathrm{dc}+\mathrm{ta}{ }^{*} \mathrm{dena}\right)$

else

$\mathrm{xbs} 1=224.29$

endif

if(xbs1.le.2.67) $\mathrm{xbs} 1=2.67$

if(xbs1.ge.224.29) xbs1 $=224.29$

bsf1 $=\operatorname{seval}(14, x b s 1, x b s$, back,bb,cb,db $)$ 
$\mathrm{C}$

C Point Geometry

C

if(igeo.eq.0)then

$\mathrm{dth}=0.0001$

$\operatorname{rmax}=0.0001$

if(isors.eq.0)SS1 $=\mathrm{ss} /\left(\mathrm{pi}^{*}\right.$ rmax $\left.^{*} \mathrm{rmax}^{*} \mathrm{dth}\right)$

FACTOR $=S S 1 * 3.7 E 4 *$ YIELD*1.6E-8*EAVE*3600./(2. $\left.{ }^{*} \mathrm{pi}^{*} \mathrm{X} 90 * \mathrm{bsf1}\right)$

C

C Disk Geometry

C

elseif(igeo.eq.1)then

$\mathrm{dth}=0.0001$

if(isors.eq.0)SS $1=\mathrm{ss} /\left(\mathrm{pi}{ }^{*} \mathrm{rmax}^{*} \mathrm{rmax}^{*} \mathrm{dth}\right)$

if (isors.eq.1) SS1 $=$ ss $/ \mathrm{dth}$

$\mathrm{C}$

FACTOR $=S S 1 * 3.7 E 4^{*}$ YIELD*1.6E-8*EAVE*3600./(2. $\left.{ }^{*} \mathrm{pi}^{*} \mathrm{X} 90^{*} \mathrm{bsf} 1\right)$

C Cylindrical Geometry

$\mathrm{C}$

elseif(igeo.eq.2)then

if(isors.eq.0)SS1 $=\mathrm{ss} /\left(\mathrm{pi}^{*} \mathrm{rmax}^{*} \mathrm{rmax}^{*} \mathrm{dth}\right)$

FACTOR $=S S 1 * 3.7 E 4 *$ YIELD*1.6E-8*EAVE*3600./(2. $\left.{ }^{*} \mathrm{pi}^{*} \mathrm{X} 90\right)$

$\mathrm{C}$

C Spherical Geometry

$\mathrm{C}$

elseif(igeo.eq.3)then

if(isors.eq.0)SS1 = (ss $\left.{ }^{*} 3.\right) /\left(4 .{ }^{*} \mathrm{pi}^{*} \mathrm{rmax}^{*} \mathrm{rmax}{ }^{*} \mathrm{rmax}\right)$

C

FACTOR $=S S 1 * 3.7 \mathrm{E} 4{ }^{*}$ YIELD*1.6E-8*EAVE*3600./(2. $\left.{ }^{*} \mathrm{pi}^{*} \mathrm{X} 90\right)$

C Slab Geometry

C

else

if(isors.eq.0)SS1 $=0.001 *$ ss / $\left(4{ }^{*}\right.$ xmax ${ }^{*}$ ymax ${ }^{*}$ dth)

FACTOR $=S S 1 * 3.7 E 7^{*}$ YIELD*1.6E-8*EAVE*3600./(4. ${ }^{*}$ pi $^{*}$ X90)

endif

$\mathrm{C}$

C Save the output file, if desired

C

if(ifonce)then

WRITE $\left({ }^{*}, 1001\right)$ filname

1001 FORMAT $(\Gamma$ Do you wish to save the output file '

$1 \quad($ File name $=$ ',a10,') $($ Default $=$ No) $? ', \$)$

$\operatorname{read}\left({ }^{*}, 3\right)$ ans

if(ans.eq.'y'.or.ans.eq.'Y')iout $=1$

ifonce $=$.false.

endif

if(iout.eq.1)then

WRITE $(*, 2)$ 
Appendix C

2 FORMAT( /20X, ' Program VARSKIN-MOD2',

1 //1X,'Enter a descriptive title for this run:' $\$$ ) $\operatorname{read}\left({ }^{*}, 31\right)$ title endif

$\mathrm{C}$

C Calculate the maximum skin depth penetration of the radiation

C

C

SkinMx $=$ Dist $-\mathrm{TC}^{*} \mathrm{DC}-\mathrm{TA}{ }^{*}$ DenA

C Initiate Volume Averaged Calculations if necessary.

C

2330 IF (ivol.eq.1)THEN

if(skinmx.le.0.)then

write $\left({ }^{*}, 2331\right)$

2331 format $(P$ The cover depth shields the beta dose completely') go to 2341

endif

$\mathrm{dmax} 1=0$.

ICount $=1$

NDep $=20$

skinmx1 = skinmx * 1000 .

DO $233 \mathrm{I}=1$,NDep

$\mathrm{XDepth}(\mathrm{I})=$ SkinMx*float $((\mathrm{I}-1) *(\mathrm{I}-1)) /$ Float $(($ NDep-1)*(ndep-1))

233 CONTINUE

vdose (ndep) $=0$.

C

ENDIF

C This is where the iteration loop begins for calculations of the

$\mathrm{C}$ volume averaged dose

C

C Evaluate the Dose at XDepth

C

234 IF (ivol.EQ.1) DMax $=$ XDepth(ICount)

C

C Check that the betas can penetrate the cover, air, and skin layers

C

2341 IF (DMax.GT.SkinMx.or.skinmx.le.0.) THEN

C

C Skin Depth is too deep... dose is zero.

$\mathrm{C}$

dos $=0$.

idos $=-1$

GOTO 1000

ENDIF

$\mathrm{C}$

C Calculate Doses for NPT pts from the center of the skin

$\mathrm{C}$ to a distance equal to a distance corresponding to

C $99 \%$ of the range of the beta

C 
411 if(abs(darea-1.).ge.0.001)bsfa $=$ bsf1

if(IGEO.LE.2)then

C

C Determine the outermost radius of irradiation on the skin

C

$\operatorname{rdmax} 1=\operatorname{sqrt}($ darea/pi $)$

depth $=\mathrm{dth}+\mathrm{ta}+\mathrm{tc}+\mathrm{dmax}$

rhomax $=$ dist $/$ dena

if(depth.gt.0.)rhomax $=$ dist*depth $/\left((\mathrm{dth}+\mathrm{ta}) *\right.$ dena $\left.+\mathrm{tc}{ }^{*} \mathrm{dc}+\mathrm{dmax}\right)$

rdmax $=$ rmax

if(rhomax.gt.depth)rdmax $=$ rmax + sqrt(rhomax* ${ }^{*}$ homax-depth*depth)

if(rdmax1.lt.rdmax)rdmax $=$ rdmax 1

elseif(IGEO.EQ.3)then

rdmax $1=\operatorname{sqrt}($ darea/pi)

$\mathrm{DEPTH}=\mathrm{RMAX}+\mathrm{TA}+\mathrm{TC}+\mathrm{DMAX}$

RHOMAX $=$ DIST $^{*}$ DEPTH $/(($ RMAX + TA $) *$ DENA + TC $*$ DC + DMAX $)$

RDMAX = RMAX + SQRT(RHOMAX*RHOMAX - DEPTH*DEPTH)

if(rdmax.gt.rdmax1)rdmax $=$ rdmax 1

elseif(IGEO.EQ.4)THEN

dpar $=0$.

write $\left({ }^{*}, 420\right)$

420 format $/ / P$ Do you wish to calculate the dose from multiple ',

1 'particles (default is No,',, Instructions will follow if',

2 'Yes) ?',\$)

$\operatorname{read}(*, 3)$ ans

if(ans.eq.'Y'.or.ans.eq.'y')then

Write $\left({ }^{*}, 421\right)$

421 Format( $/$ VARSKIN Mod 2 offers the option to calculate doses',

1 'from multiple particles.'; $;$ To do so, the dose from each',

2 'particle must be calculated separately.', First, choose',

3 'the location of the CENTER of the area over which to average ',

$4 /$ the dose. Second, determine the location of the CENTER of ',

5 'the particle', $\Gamma$ for which the dose is to be calculated. Now',

6 ' enter the distance', $P$ (offset) between these two points in',

7 'um :'\$)

read $\left({ }^{*}, 5\right)$ dpar1

dpar $=$ dpar $1 / 10000$.

ipar $=1$

If(xmax1-ymax1.gt.0.27)then

xsugg $=\operatorname{sqrt}(x \max 1 * \mathrm{ymax} 1)$

Write $\left({ }^{*}, 422\right) x s u g g, x s u g g$

422 format(' For this model, the X-side length must equal the ',

1 'Y-side length.', $f$ The suggested value is ',f6.0,' um.',

2 ' Please enter $a^{\prime}, f$ new side length in um (or <enter $>$ ',

3 'to accept ',f6.0,' um) : ',\$)

$\operatorname{read}\left({ }^{*}, 5\right)$ xmax1

if(xmax1.le.0.) $\mathrm{xmax} 1=\mathrm{xsugg}$

$\mathrm{ymax} 1=\mathrm{xmax} 1$

$\mathrm{xmax}=\mathrm{xmax} 1 / 20000$. 
Appendix C

$y \max =y \max 1 / 20000$.

if(isors.eq.0)SS1 $=0.001{ }^{*} \mathrm{ss} /\left(4{ }^{*} \mathrm{xmax}^{*} \mathrm{ymax}^{*} \mathrm{dth}\right)$

FACTOR $=S S 1 * 3.7 E 7 *$ YIELD*1.6E-8*EAVE*3600./(4. ${ }^{*}$ pi $^{*}$ X90)

EndIf

endif

GO TO 503

ENDIF

C

DO $500 \mathrm{I}=1, \mathrm{NPT}$

C PS is the distance to the dose point from the center of the skin

$\mathrm{C}$

$\mathrm{PS}=\mathrm{FLOAT}(\mathrm{I}-1) / \mathrm{FLOAT}(\mathrm{NPT}-1){ }^{*} \operatorname{rdmax}$

if(rmax/rdmax.le.0.15)

$1 \mathrm{PS}=(\mathrm{FLOAT}(\mathrm{I}-1) / \mathrm{FLOAT}(\mathrm{NPT}-1) * \operatorname{sqrt}(\mathrm{rdmax}))^{* * 2}$

if(rmax/rdmax.ge.0.85)

1 PS $=\operatorname{sqrt}\left(\right.$ FLOAT(I-1)/FLOAT(NPT-1) $\left.{ }^{*} \operatorname{rdmax}{ }^{*} \operatorname{rdmax}\right)$

$\mathrm{XB}(\mathrm{I})=\mathrm{PS}$

if(i.eq.1)write $\left({ }^{*}, 5000\right)$

5000 format $(P$ Performing Calculations')

IF(IGEO.EQ.3)GO TO 502

radmax $=\operatorname{rmax}$

$\mathrm{a} 3=0.000027$

b3 $=$ radmax

depth $=\mathrm{dth}+\mathrm{ta}+\mathrm{tc}+\mathrm{dmax}$

rhomax $=$ dist $/$ dena

rhomax $=$ dist ${ }^{*}$ depth/((dth + ta $) *$ dena $\left.+\mathrm{tc}^{*} \mathrm{dc}+\mathrm{dmax}\right)$

if(rhomax.gt.depth)a3=ps-sqrt(rhomax*rhomax-depth*depth)

if(rhomax.gt.depth)b3=ps + sqrt(rhomax*rhomax-depth*depth)

if(a3.lt.0.000027) $\mathrm{a} 3=0.000027$

if(b3.gt.radmax $) \mathrm{b} 3=$ radmax

$\mathrm{A} 4=0.0$

$\mathrm{B} 4=\mathrm{pi}$

a5 $=0.0$

$\mathrm{b} 5=\mathrm{dth}$

DDOSE = QUAD3(dum1,dum2,arg1)

go to 504

$502 \mathrm{~A} 3=0.000027$

$\mathrm{B} 3=\mathrm{RMAX}$

$\mathrm{A} 4=0.0$

$\mathrm{B} 4=\mathrm{pi}$

A5 $=0.0$

$\mathrm{B} 5=\mathrm{pi}$

DDOSE = QUAD3(dum1,dum2,arg2)

504 DDOSE $=$ DDOSE $*$ bsfa

$\operatorname{DOSE}(\mathrm{I})=$ FACTOR $*$ DDOSE

IF ( DOSE(I) .LT. 0. ) DOSE(I) $=0$.

500 CONTINUE

GO TO 1000

C 


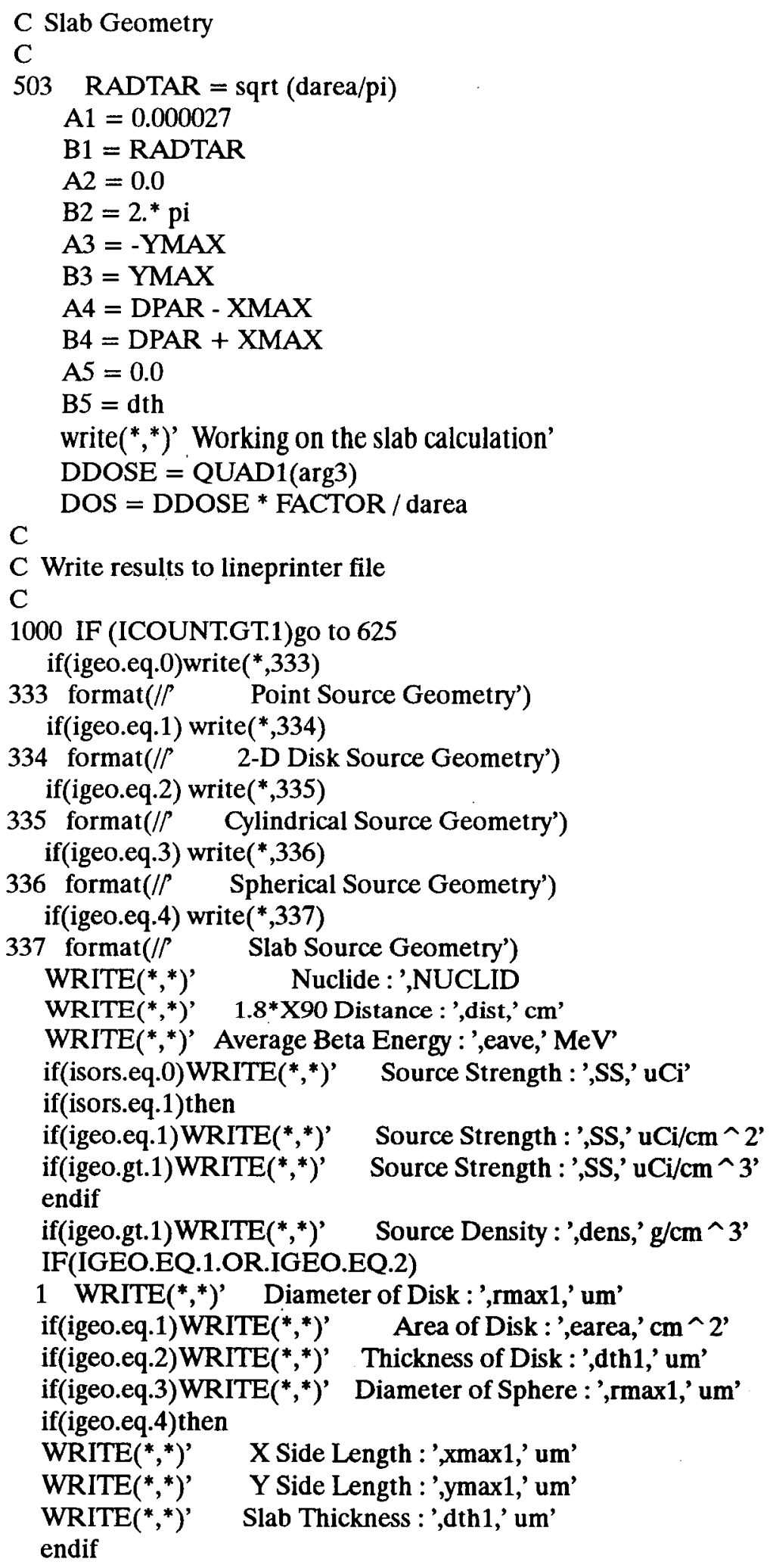


if(ipar.eq.1)WRITE $\left({ }^{*},{ }^{*}\right)$ ' Particle Offset : ',dpar1,' um'

WRITE $\left({ }^{*},{ }^{*}\right)$ Skin Depth :',dmax1,' mg/cm^^2'

WRITE $\left({ }^{*},{ }^{*}\right)$ ' Thickness of Cover : ',tc1,' mm'

if(tc1.gt.0.)WRITE $\left({ }^{*},{ }^{*}\right)$ ' Cover Density : ',dc,' g/cm^ 3 '

WRITE $\left({ }^{*},{ }^{*}\right)$ ' Air Gap Thickness : ',ta1,' mm'

WRITE $\left({ }^{*},{ }^{*}\right)$ ' Irradiation Time : ',ttime,' min'

if(iout.eq.1)then

OPEN $($ UNIT $=15$, FILE $=$ FILNAME, STATUS $=$ 'NEW'

WRITE $(15,6)$ title

6 FORMAT( /20X, ' Program VARSKIN-MOD2',//a,/)

if(igeo.eq.0)write(15,*)' Point Source Geometry'

if(igeo.eq.1) write $\left(15,{ }^{*}\right)$ ' 2-D Disk Source Geometry'

if(igeo.eq.2) write $\left(15,{ }^{*}\right)$ ' Cylindrical Source Geometry'

if(igeo.eq.3) write $\left(15,{ }^{*}\right)$ ' Spherical Source Geometry'

if(igeo.eq.4) write $\left(15,{ }^{*}\right)^{\prime} \quad$ Slab Source Geometry'

WRITE $\left(15,{ }^{*}\right)$

WRITE $(15, *)$

WRITE $\left(15,{ }^{*}\right)$ ' Nuclide : ',NUCLID

WRITE $\left(15,{ }^{*}\right)^{\prime} \quad 1.8^{*}$ X90 Distance : ',dist,' cm'

WRITE $\left(15,{ }^{*}\right)$ ' Average Beta Energy : ',eave,' MeV'

if(igam.eq.1)then

write $\left(15,{ }^{*}\right)$ ' Average Gamma Energy : ',geavg,' keV'

write $\left(15,{ }^{*}\right)$ ' Gamma Factor :',gamfac,' R-cm^^2/mCi-h'

else

write $\left(15,{ }^{*}\right)^{\prime} \quad$ No gamma dose calculation'

endif

if(isors.eq.0)WRITE $\left(15,{ }^{*}\right)$ ' Source Strength : ',SS,' uCi'

if(isors.eq.1)then

if(igeo.eq.1)WRITE $\left(15,{ }^{*}\right)$ ' Source Strength : ',SS,' uCi/cm ^2'

if(igeo.gt.1)WRITE $\left(15,{ }^{*}\right)$ ' Source Strength : ',SS,' uCi/cm^^3'

endif

IF(IGEO.GT.1)WRITE(15, $\left.{ }^{*}\right)$ Source Density : ',dens,' g/cm ^3'

IF(IGEO.EQ.1.OR.IGEO.EQ.2)

1 WRITE $\left(15,{ }^{*}\right)$ ' Diameter of Disk : ',rmax1,' um'

if(igeo.eq.1)WRITE $\left(15,{ }^{*}\right)$ ' $\quad$ Area of Disk : ',earea,' $\mathrm{cm}^{\wedge} 2$ '

IF(IGEO.EQ.2)WRITE $\left(15,{ }^{*}\right)$ ' Thickness of Disk : ',dth1,' um'

IF(IGEO.EQ.3)WRITE $\left(15,{ }^{*}\right)$ ' Diameter of Sphere : ',rmax1,' um'

IF(IGEO.EQ.4)THEN

WRITE $\left(15,{ }^{*}\right)^{\prime} \quad X$ Side Length : ',xmax 1 ,' um'

WRITE $\left(15,{ }^{*}\right)$ ' Y Side Length : ',ymax1,' um'

WRITE $\left(15,{ }^{*}\right)$ ' Thickness : ',dth1,' um'

if(ipar.eq.1)WRITE $\left(15,{ }^{*}\right)$ ' Particle Offset : ',dpar1,' um'

ENDIF

WRITE $\left(15,{ }^{*}\right)^{\prime} \quad$ Skin Depth : ',dmax1,' $\mathrm{mg} / \mathrm{cm}$ ^2'

WRITE $\left(15,{ }^{*}\right)$ ' Thickness of Cover : ',tc1,' mm'

if(tc1.gt.0.)WRITE(15, $\left.{ }^{*}\right)$ Cover Density : ',dc,' g/cm ^3'

WRITE $\left(15,{ }^{*}\right)$ ' Air Gap Thickness : ',ta1,' mm'

WRITE $\left(15,{ }^{*}\right)$ ' Irradiation Time : ',ttime,' min'

if(idos.lt.0)go to 1100 
if(IGEO.EQ.4)go to 1100

if(IVOL.EQ.1)go to 625

WRITE $(15,600)$

600 FORMAT(/' Calculated Results:',

1 If Radial ',6X,' Dose ', $P$ Distance', 6x,' Rate',

2 P (cm) ',6x,' (rad/hr)',P -...--.-',6X,'---.-...')

DO $610 \mathrm{I}=1, \mathrm{npt}$

610 WRITE $(15,620) \mathrm{XB}(\mathrm{I}), \mathrm{DOSE}(\mathrm{I})$

620 FORMAT( OpF10.4, 1pe16.2)

ENDIF

625 if(idos.lt.0)go to 1100

$\mathrm{C}$

if(IGEO.EQ.4)go to 1100

C Calculate the dose rate averaged over the radius of the target sphere

$\mathrm{C}$

C First, spline-fit the calculated dose-distance data for the

$\mathrm{C}$ depth dose points

C

C

CALL SPLINE(npt,xb,dose,Bx,Cx,Dx1)

C Evaluate the spline for 100 points over the target radius

C STEP is the step size for the averaging.

C

C Set the target radius for the average

C

630 radtar $=$ sqrt $($ darea/pi $)$

radtem $=$ radtar

if(radtar.gt.xb(npt))radtar $=x b(n p t)$

step $=(\operatorname{radtar}-x b(1)) / 99$.

dostar $=$ seval(npt,radtar,xb,dose,bx,cx,dx1)

dos $=$ radtar ${ }^{*}$ dostar

Do $640 \mathrm{I}=1,49$

stepo $=$ float $(2 * \mathrm{i}-1){ }^{*}$ step

C

stepe $=$ float $(2 * i) *$ step

C Use Simpson's $1 / 3$ rule to calculate the average dose

C

aone $=\operatorname{seval}(\mathrm{npt}, \mathrm{stepo}, \mathrm{xb}, \mathrm{dose}, \mathrm{bx}, \mathrm{cx}, \mathrm{dx} 1)$

bone $=\operatorname{seval}(\mathrm{npt}, \mathrm{stepe}, \mathrm{xb}, \mathrm{dose}, \mathrm{bx}, \mathrm{cx}, \mathrm{dx1})$

dos $=$ dos $+4 .{ }^{*}$ stepo*aone $+2 .{ }^{*}$ stepe $*$ bone

640 continue

$\mathrm{C}$

C Complete the calculation of the averages

$\mathrm{C}$

C

$\operatorname{dos}=2 .{ }^{*} \operatorname{dos}{ }^{*} \operatorname{step} /\left(3{ }^{*}\right.$ radtem ${ }^{*}$ radtem $)$

C Write the results to the lineprinter file

C

if(xb(npt).lt.radtem)then 
Appendix C

dos $1=$ dos ${ }^{*}$ radtem ${ }^{*}$ radtem $/ \mathrm{xb}(\mathrm{npt}) / \mathrm{xb}(\mathrm{npt})$

write $\left({ }^{*}, 649\right) \times b(n p t)$, dos 1

if(iout.eq.1)write(15,649)xb(npt),dos1

649 format $(\rho$ The dose averaged over the area of irradiation',

$1 \rho($ radius $=$ ',f10.4,' cm $)=$ ',1 pe10.2,' $\mathrm{rad} / \mathrm{hr}$ ')

else

write $(*, 648)$ darea

if(iout.eq.1)write $(15,648)$ darea

648 format $(\rho$ The area of irradiation is larger than ',f10.4,

1' square cm')

endif

1100 write $\left({ }^{*}, 650\right)$ darea,dos

if(iout.eq.1)write $(15,650)$ darea,dos

650 format $(P$ The beta dose rate averaged over ',f10.4,' square cm',

1 '=',

$\left.11 \mathrm{pe} 10.2, \mathrm{rad}^{\mathrm{h}} \mathrm{hr}\right)$

dost $=$ ttime ${ }^{*}$ dos $/ 60$.

write $(*, 6601)$ darea,dost

if(iout.eq.1)write $(15,6601)$ darea,dost

6601 format $(P$ The total beta dose averaged over ',f10.4,' square cm',

$\mathrm{C}$ $1 '=$ ',1pe10.2,' rad')

C Continue the volume averaging routine

C Loop to line 234 to calculate another dose, if necessary.

C

IF (ivol.EQ.1) THEN

write $\left({ }^{*}, 6499\right)$

6499 format(/P Volume-Averaged Dose Model')

VDose $($ ICount $)=$ Dos

ICount $=$ ICount +1

DMax $=$ XDepth(ICount)

IF (ICount.LT.NDep) THEN

percnt $=100 *$ Float $($ ICount-1)/FLoat(NDep-1)

WRITE $(*, 6500)$ percnt

6500 format (2x,f6.2,' \% complete.',/)

GOTO 234

ELSE

WRITE $(*, 6501)$

6501 format(' Intial Calculations are complete')

ENDIF

C

C Compute Spline Coefficients for VDose array

$\mathrm{C}$

WRITE $(*, 651)$

if(iout.eq.1)WRITE(15,651)

651 format(' DEPTH $\left(\mathrm{mg} / \mathrm{cm}^{\wedge} 2\right)$ DOSE $\left.(\mathrm{rad} / \mathrm{h})^{\prime}\right)$

DO $1111 \mathrm{I}=1, \mathrm{NDEP}$

xdepa $=1000{ }^{*}$ xdepth $(\mathrm{i})$

WRITE $(*, 652)$ XDEPA, VDOSE(I) 
if(iout.eq.1)WRITE(15,652)XDEPTH(I),VDOSE(I)

652 format(5x,1pe10.2,15x,1pe10.2)

1111 CONTINUE C

CALL SPLINE(NDep,XDEPTH,VDOSE,VB,VC,VD)

C Ask the user for the range of values over which to compute the

C volume-averaged dose.

C

1112 Write $\left({ }^{*}, 11121\right)$ skinmxi

11121 format $(/ /$ The range of values allowed for calculating the'

1 'volume-averaged dose is 0 to ',f10.3, $P$ Enter the depth ',

2 ' $(\mathrm{mg} / \mathrm{cm} 2)$ corresponding to the top of the cell',

$3 P$ (the shallower depth) over',

4 'which to average the dose (default is 0 ): ' $\$$ )

$\operatorname{read}\left({ }^{*}, 5\right)$ xlow1

if(xlow1.It.0.)then

write $\left({ }^{*}, 11123\right)$

11123 format ( $\rho$ ERROR: The shallow depth must be non-negative')

go to 1112

endif

if(xlow1.ge.skinmx1)then

write $\left({ }^{*}, 21123\right)$ skinmx1

21123 format( $/$ ERROR: The shallow depth must be less than ',f10.3)

go to 1112

endif

xlow $=$ xlow $1 / 1000$.

11124 Write $\left({ }^{*}, 11122\right)$ skinmx1

11122 format $(/ /$ Enter the depth $(\mathrm{mg} / \mathrm{cm} 2)$ corresponding to the ',

1 'bottom of the cell', $f$ (the deeper depth) over',

2 'which to average the dose (maximum is ',f10.3,'): '\$)

read $\left({ }^{*}, 5\right) \times$ high 1

if(xhigh1.le.0.)xhigh1 = skinmx1

if(xhigh1.le.xlow1)then

write $\left({ }^{*}, 11125\right)$

11125 format $(P$ The deep depth must be greater than the shallow depth')

go to 11124

endif

if(xhigh1.gt.skinmx1)then

write $\left({ }^{*}, 11127\right)$ skinm $x 1$

11127 format $(P$ The deep depth must be less than ',f10.3)

go to 11124

endif

C

xhigh $=$ xhigh $1 / 1000$.

C Use Simpson's $1 / 3$ rule to evaluate the dose between xlow and xhigh

C

step $=($ xhigh $-x$ low $) / 99$.

doslow = seval(ndep,xlow,xdepth,vdose,vb,vc,vd)

doshi = seval(ndep,xhigh,xdepth,vdose,vb,vc,vd) 
Appendix C

$$
\begin{aligned}
& \text { dos }=\text { doslow }+ \text { doshi } \\
& \text { Do } 1640 \mathrm{I}=1,49 \\
& \text { stepo }=\text { xlow }+ \text { float }(2 * \mathrm{i}-1) * \text { step }
\end{aligned}
$$


$\mathrm{C}$

if (gdepth.le.0.)gdepth $=0.000027$

gdos1 $=$ pi $^{*}$ gamfac*alog $^{*} 1 .+$ grad $\left.^{*} \mathrm{grad} / \mathrm{gdepth} / \mathrm{gdepth}\right)$

C Electronic Equilibrium Model

C

gdep1 $=\mathrm{dmax}+\mathrm{ta} *$ dena $+\mathrm{tc}^{*} \mathrm{dc}$

if(gdep1.le.0.02)then

afac $=0.129+0.000338 *$ geavg

bfac $=171.8 * \exp (-0.00162 *$ geavg $)$

else

afac $=0.062+0.0003055^{*}$ geavg

bfac $=156.0 * \exp \left(-0.00193^{*}\right.$ geavg $)$

endif

eleceq $=1$. - afac ${ }^{*} \exp \left(-\mathrm{bfac}{ }^{*}\right.$ gdepth $)$

ssg $=$ ss

if(isors.eq.1.and.igeo.le.2)ssg $=\mathrm{ss} 1{ }^{*} \mathrm{pi}^{*}{ }^{*} \mathrm{rmax}^{*} \mathrm{rmax}^{*} \mathrm{dth}$

if(isors.eq.1.and.igeo.eq.3)ssg $=\mathrm{ss} 1 * 4 .{ }^{*} \mathrm{pi}^{*}{ }^{*} \max ^{*} \mathrm{rmax}{ }^{*} \mathrm{rmax} / 3$.

if(isors.eq.1.and.igeo.eq.4)ssg $=4{ }^{*}{ }^{*} \mathrm{ss} 1{ }^{*} \mathrm{xmax}{ }^{*} \mathrm{ymax}{ }^{*} \mathrm{dth}$

gamdos $=(\mathrm{SSG} / 1000)$.$* gdos 1 *$ eleceq $* 0.96$

if(igam.eq.1)write $\left({ }^{*}, 655\right)$ darea,gamdos

if(iout.eq.1.and.igam.eq.1)write $(15,655)$ darea,gamdos

655 format ( $P$ The gamma dose rate averaged over ',f10.4,' square cm'

1 ' = ',1 pe10.2,' $\mathrm{rad} / \mathrm{hr}$ ')

gdost $=$ ttime ${ }^{*}$ gamdos $/ 60$.

if(igam.eq.1)write $\left({ }^{*}, 656\right)$ darea,gdost

if(iout.eq.1.and.igam.eq.1)write(15,656)darea,gdost

656 format $(r$ The total gamma dose averaged over ',f10.4,' square cm'

$1^{\prime}$ = ',1pe10.2,' rad')

701 if(nnuc.gt.1.and.iint.eq.0.and.ivol.eq.0)then

dosrt $=$ dosrt + dos

if(igam.eq.1) gdosrt $=$ gdosrt + gamdos

iint $=1$

endif

darea $=0$.

igam $=0$

idos $=0$

if(jj.eq.nnuc.and.nnuc.eq.1)then

write $\left({ }^{*}, 660\right)$

660 format $(\rho$ Enter new area $(\mathrm{cm} \wedge 2)$ over which to average dose'

$1 \rho$ or $<$ RETURN $>$ to exit VARSKIN Mod 2: '\$) elseif(jj.eq.nnuc.and.nnuc.gt.1)then

write $\left({ }^{*}, 667\right)$ nnuc

667 format $\left(P\right.$ Enter new area $\left(\mathrm{cm}^{\wedge} 2\right)$ over which to average dose or '

1 '<RETURN $>$ to view the total doses from the ',i1,' nuclides',\$) else

write $(*, 661)$

661 format $\left(\rho\right.$ Enter new area $\left(\mathrm{cm}^{\wedge} 2\right)$ over which to average dose'

1 'or $<$ RETURN $>$ for next nuclide: '\$) endif 
Appendix C

$\operatorname{read}\left({ }^{*}, 5\right)$ darea

if(darea.le.0.)then

if(jj.eq.nnuc.and.nnuc.gt.1)then

dostot $=$ dosrt ${ }^{*}$ ttime $/ 60$.

if(gdosrt.gt.0.)gdostt $=$ gdosrt * ttime $/ 60$.

write $\left({ }^{*}, 662\right)$ ttime,nnuc, dosrt

if(iout.eq.1)write $(15,662)$ ttime,nnuc,dosrt

662 format(/Irradiation time = ',1pe10.1,' min', $/$ The beta dose ',

1 'rate for the ',i1,' radionuclides, averaged over',; 1 square',

2' cm, = ',1pe10.2,' $\mathrm{rad} / \mathrm{hr}$ ')

write $\left({ }^{*}, 663\right)$ nnuc,dostot

if(iout.eq.1)write(15,663)nnuc,dostot

663 format $(P$ The total beta dose for the ',il,' radionuclides, ',

\& 'averaged over', $\rho 1$ square $\mathrm{cm},=$,, 1 pe10.2,' rad')

if(gdosrt.gt.0.)then

write $\left({ }^{*}, 664\right)$ nnuc,gdosrt

if(iout.eq.1)write(15,664)nnuc,gdosrt

664 format $(\rho$ The gamma dose rate for the ',i1,' radionuclides, ',

\& 'averaged over', $f 1$ square $\mathrm{cm},=$ ',1 pe10.2,' $\mathrm{rad} / \mathrm{hr}$ ')

write $(*, 665)$ nnuc,gdostt

if(iout.eq.1)write $(15,665)$ nnuc, gdostt

665 format $(/$ The total gamma dose for the ',i1,' radionuclides, ', \& 'averaged over',, 1 square $\mathrm{cm},=$ ',1pe10.2,' rad')

endif

write $\left({ }^{*}, 668\right)$

668 format $(>$ Hit $<$ ENTER $>$ when you are ready to exit VARSKIN Mod 2',

\&\$)

$\operatorname{read}(*, 5)$

endif

iint $=0$

go to 10

endif

if(igeo,eq.4)go to 503

radtr2 $=$ sqrt $($ darea/pi)

if(ivol.eq.1)go to 2330

if $(x b(n p t)$.It.radtem.and.xb(npt).lt.radtr2)then

dos $=\operatorname{dos}^{*}$ radtem ${ }^{*}$ radtem $/$ radtr $2 /$ radtr 2

radtem $=$ radtr 2

go to 1100

endif

if (radtr2.gt.radtem)go to 411

go to 630

10 continue

if(iout.eq.1)close(15)

if(iout.eq.1)Write $\left({ }^{*}, 681\right)$ filname

681 format ( $P$ ',a10,' has been saved to disk.')

800 write $(*, 690)$

690 FORMAT( $($ End of Varskin-Mod2')

STOP 
END

C

$\mathrm{C}^{* * * * * * * * * * * * * * * * * * * * * * * * * * * * * * * * * * * * * * * * * * * * * * * * * * * * * * * * * * *}$

C

SUBROUTINE READX(Default,X)

C This subroutine Reads upto 12 characters from the keyboard and

C returns REAL*4 Default value of X if the RETURN key is pressed.

$C$ Otherwise the characters are converted to a real number and

$C$ returned in $X$. R.D. Stewart 4-11-91

REAL*4 X,Default

CHARACTER*12 Ans

1 FORMAT(A12)

$\operatorname{READ}\left({ }^{*}, 1\right)$ Ans

IF (Ans.EQ.' ') THEN

C Use Default Value

$\mathrm{X}=$ Default

ELSE

READ(Ans,'(F12.0)') X

ENDIF

RETURN

END

C

$C^{* * * * * * * * * * * * * * * * * * * * * * * * * * * * * * * * * * * * * * * * * * * * * * * * * * * * * * * * * * * * * * * * * * * * * *}$

C

REAL FUNCTION QUAD1( ARG )

EXTERNAL ARG

DIMENSION F(8),X(8),XSAVE(4,30),FSAVE(4,30),QSAVE(30)

COMMON /QUADS/ A1,A2,A3,A4,A5,B1,B2,B3,B4,B5,RELERR

PARAMETER $(\mathrm{W} 0=0.3111111111, \mathrm{~W} 1=1.4222222222, \mathrm{~W} 2=0.5333333333$ )

PARAMETER $(E 0=0.279082892, E 1=1.661516755, E E 2=0.261869489)$

C

PARAMETER(E3 $=2.961834215, E E 4=1.281128748)$

$$
\begin{aligned}
& \mathrm{E} 2=-\mathrm{EE} 2 \\
& \mathrm{E} 4=-\mathrm{EE} 4 \\
& \mathrm{~A}=\mathrm{A} 1 \\
& \mathrm{~B}=\mathrm{B} 1
\end{aligned}
$$

C

ANS $=0$.

AREA $=0$.

C

QPREV $=0$.

C

$\mathrm{LEV}=0$

$\mathrm{X} 0=\mathrm{A}$

$\mathrm{X}(8)=\mathrm{B}$

$\mathrm{F} 0=\mathrm{QUAD} 2(\mathrm{x} 0, \mathrm{ARG})$

$\mathrm{X}(4)=(\mathrm{X} 0+\mathrm{X}(8))^{*} 0.5$

$\mathrm{X}(2)=(\mathrm{X} 0+\mathrm{X}(4)) * 0.5$

$\mathrm{X}(6)=(\mathrm{X}(4)+\mathrm{X}(8)) * 0.5$ 
Appendix C

C

$\mathrm{DO} 25 \mathrm{~J}=2,8,2$

$\mathrm{F}(\mathrm{J})=\mathrm{QUAD} 2(\mathrm{X}(\mathrm{J}), \mathrm{ARG})$

25 CONTINUE

$30 \mathrm{X}(1)=(\mathrm{X} 0+\mathrm{X}(2)) * 0.5$

$F(1)=Q U A D 2(X(1), A R G)$

DO $35 \mathrm{~J}=3,7,2$

$\mathrm{X}(\mathrm{J})=(\mathrm{X}(\mathrm{J}-1)+\mathrm{X}(\mathrm{J}+1))^{*} 0.5$

$F(J)=Q U A D 2(X(J), A R G)$

35 CONTINUE

C

$\mathrm{STEP}=(\mathrm{X}(8)-\mathrm{X} 0) / 8$.

$\mathrm{QRIGHT}=\left(\mathrm{W} 0 *(\mathrm{~F}(4)+\mathrm{F}(8))+\mathrm{W} 1^{*}(\mathrm{~F}(5)+\mathrm{F}(7))+\mathrm{W} 2 * \mathrm{~F}(6)\right) * \mathrm{STEP}$

$\mathrm{QLEFT}=(\mathrm{W} 0 *(\mathrm{~F} 0+\mathrm{F}(4))+\mathrm{W} 1 *(\mathrm{~F}(1)+\mathrm{F}(3))+\mathrm{W} 2 * \mathrm{~F}(2)) * \mathrm{STEP}$

$\mathrm{EIGHT}=(\mathrm{E} 0 *(\mathrm{~F} 0+\mathrm{F}(8))+\mathrm{E} 1 *(\mathrm{~F}(1)+\mathrm{F}(7))+\mathrm{E} 2 *(\mathrm{~F}(2)+\mathrm{F}(6))+$

$1 \mathrm{E} 3 *(\mathrm{~F}(3)+\mathrm{F}(5))+\mathrm{E} 4 * \mathrm{~F}(4)){ }^{*} \mathrm{STEP}$

C

QADD $=$ QLEFT + QRIGHT

DIFF $=$ QADD-QPREV

C

AREA $=$ AREA+DIFF

C

IF( ABS(QADD-EIGHT) .LE. RELERR*ABS(AREA) ) GOTO 99

C

$\mathrm{SIM}=\mathrm{STEP}^{*}\left(\mathrm{~F}(6)+\mathrm{F}(4)+4 .^{*}(\mathrm{~F}(7)+\mathrm{F}(5))+\mathrm{F}(8)+\mathrm{F}(6)\right) / 3$.

C

IF( ABS(QRIGHT-SIM) .LE. RELERR*ABS(AREA) ) GOTO 60

$\mathrm{LEV}=\mathrm{LEV}+1$

C

IF(LEV.GE.30)GOTO900

FSAVE $(1, \mathrm{LEV})=\mathrm{FO}$

$\mathrm{XSAVE}(1, \mathrm{LEV})=\mathrm{X} 0$

DO $52 \mathrm{I}=2,4$

FSAVE $(\mathrm{I}, \mathrm{LEV})=\mathrm{F}(\mathrm{I}-1)$

XSAVE $(I, L E V)=X(I-1)$

52 CONTINUE

QSAVE $($ LEV $)=$ QLEFT

C

$\mathrm{X} 0=\mathrm{X}(4)$

$\mathrm{F} 0=\mathrm{F}(4)$

C

DO $55 \mathrm{I}=1,3$

$\mathrm{F}\left(2^{*} \mathrm{I}\right)=\mathrm{F}(4+\mathrm{I})$

$\mathrm{X}\left(2^{*} \mathrm{I}\right)=\mathrm{X}(4+\mathrm{I})$

55 CONTINUE

QPREV $=$ QRIGHT

GOTO30

60 ANS $=$ ANS + QRIGHT

DO $65 \mathrm{I}=4,1,-1$ 


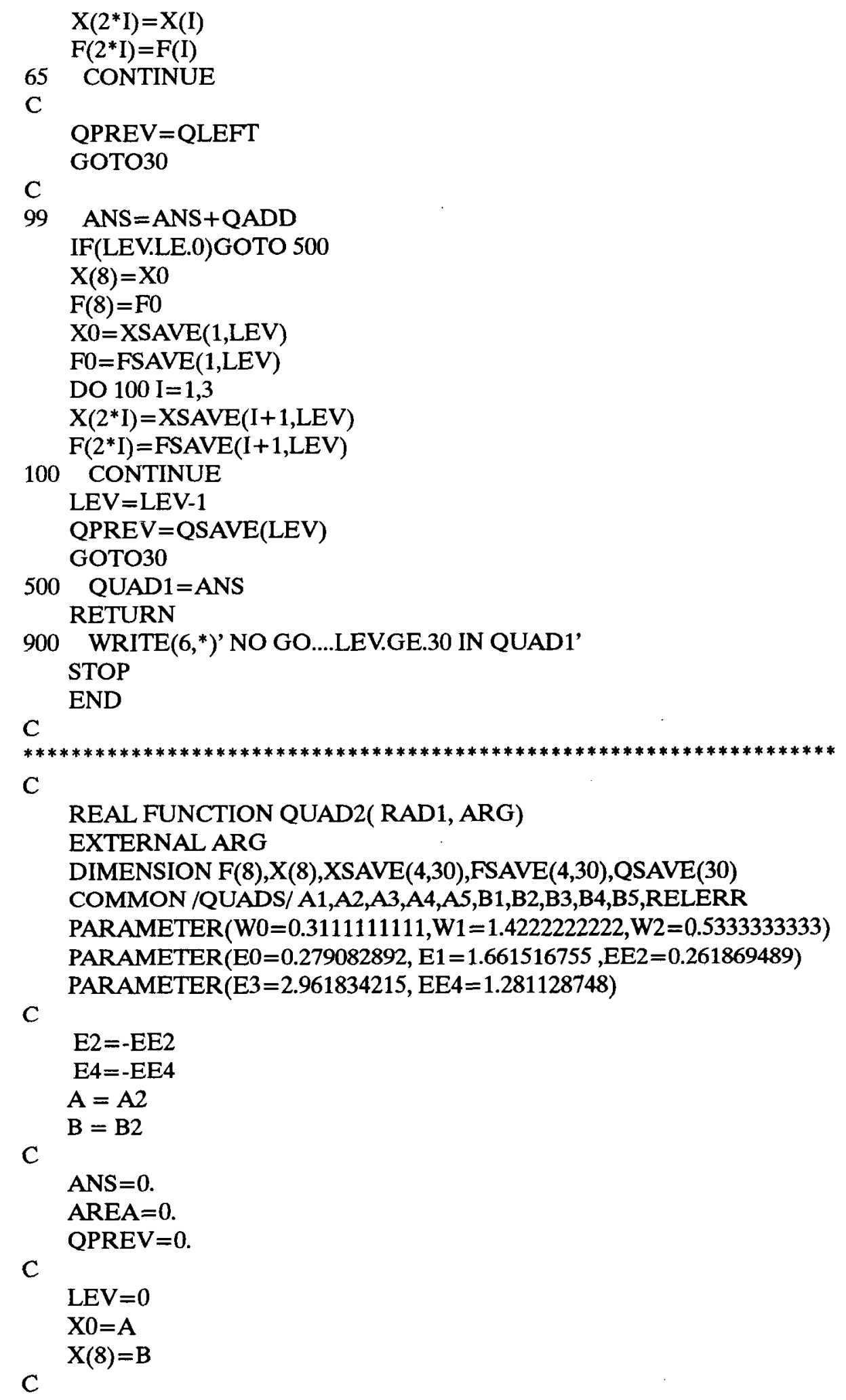


Appendix C

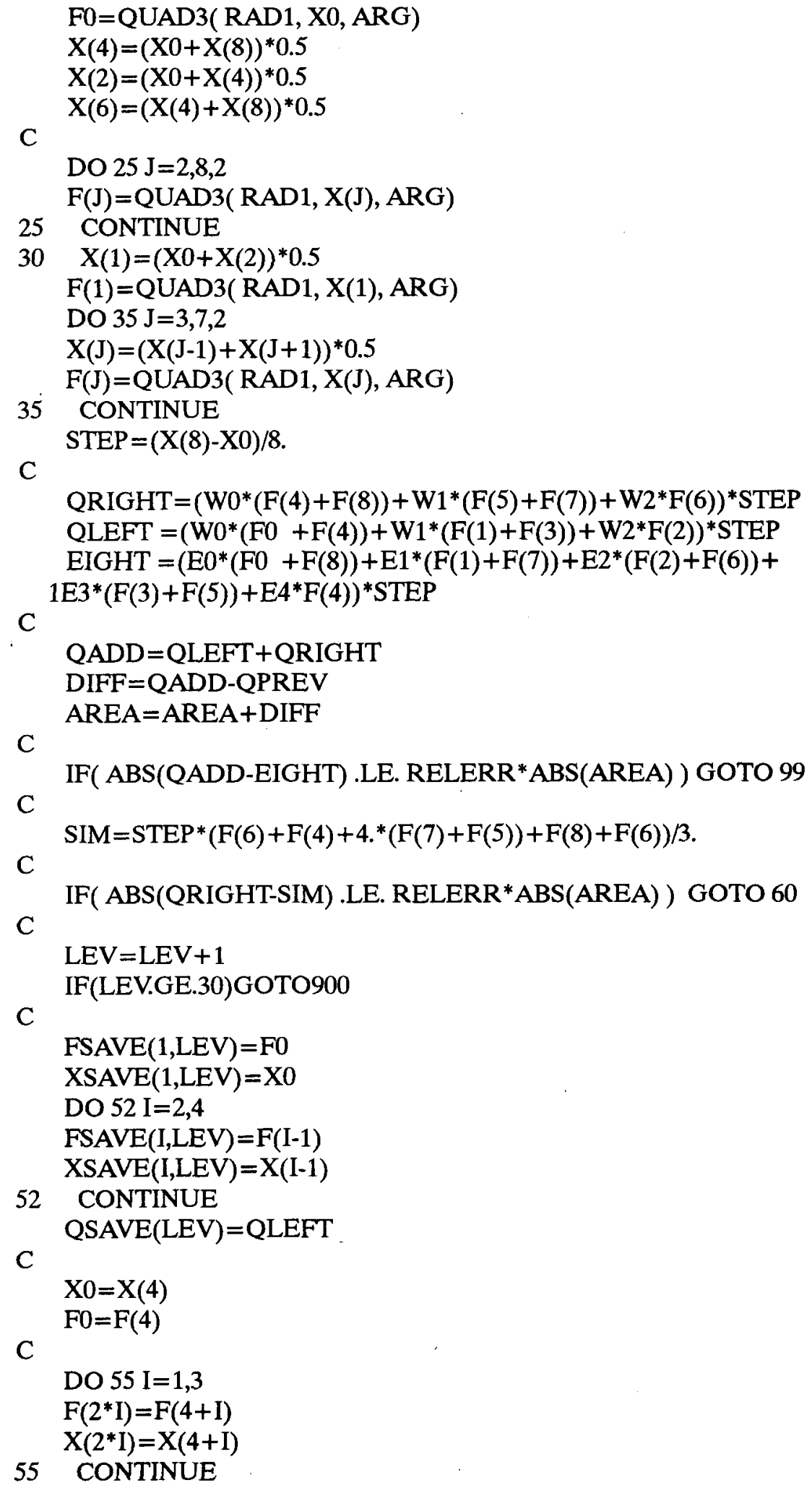




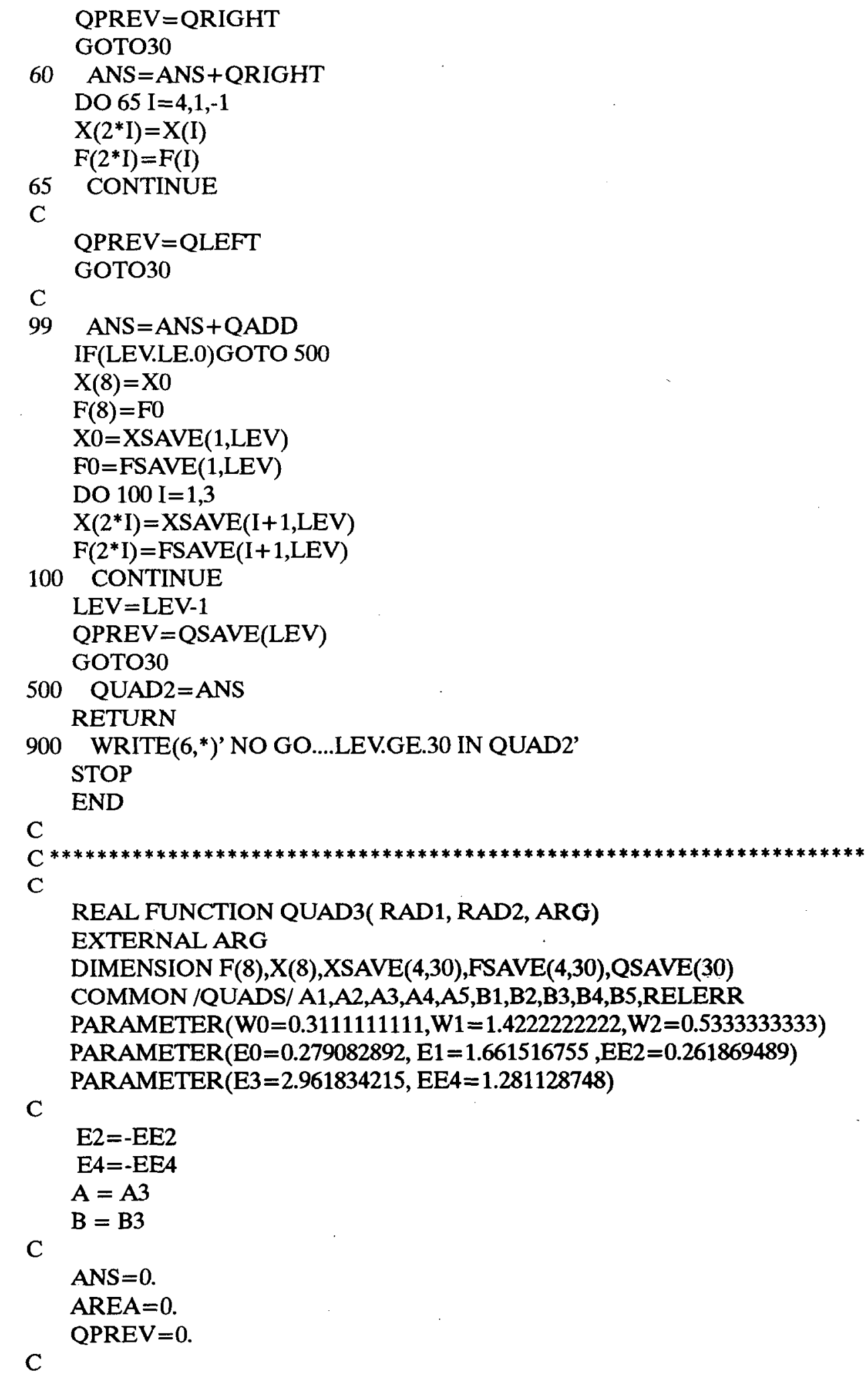


Appendix C

$\mathrm{LEV}=0$

$\mathrm{X} 0=\mathrm{A}$

C

$X(8)=B$

$\mathrm{F} 0=\mathrm{QUAD} 4(\mathrm{X} 0, \mathrm{RAD} 1, \mathrm{RAD} 2, \mathrm{ARG})$

$\mathrm{X}(4)=(\mathrm{X} 0+\mathrm{X}(8)) * 0.5$

$X(2)=(X 0+X(4)) * 0.5$

C

$\mathrm{X}(6)=(\mathrm{X}(4)+\mathrm{X}(8)) * 0.5$

DO $25 \mathrm{~J}=2,8,2$

$\mathrm{F}(\mathrm{J})=\mathrm{QUAD} 4(\mathrm{X}(\mathrm{J}), \mathrm{RAD} 1, \mathrm{RAD} 2, \mathrm{ARG})$

25 CONTINUE

$30 \mathrm{X}(1)=(\mathrm{X} 0+\mathrm{X}(2)) * 0.5$

$\mathrm{F}(1)=\mathrm{QUAD} 4(\mathrm{X}(1), \mathrm{RAD} 1, \mathrm{RAD} 2, \mathrm{ARG})$

DO $35 \mathrm{~J}=3,7,2$

$\mathrm{X}(\mathrm{J})=(\mathrm{X}(\mathrm{J}-1)+\mathrm{X}(\mathrm{J}+1)) * 0.5$

$\mathrm{F}(\mathrm{J})=\mathrm{QUAD} 4(\mathrm{X}(\mathrm{J}), \mathrm{RAD} 1, \mathrm{RAD} 2, \mathrm{ARG})$

35 CONTINUE

$\mathrm{STEP}=(\mathrm{X}(8)-\mathrm{X} 0) / 8$.

C

$\mathrm{QRIGHT}=(\mathrm{W} 0 *(\mathrm{~F}(4)+\mathrm{F}(8))+\mathrm{W} 1 *(\mathrm{~F}(5)+\mathrm{F}(7))+\mathrm{W} 2 * \mathrm{~F}(6)) * \mathrm{STEP}$

QLEFT $=(\mathrm{W} 0 *(\mathrm{~F} 0+\mathrm{F}(4))+\mathrm{W} 1 *(\mathrm{~F}(1)+\mathrm{F}(3))+\mathrm{W} 2 * \mathrm{~F}(2)) * \mathrm{STEP}$

$\mathrm{EIGHT}=(\mathrm{E} 0 *(\mathrm{~F} 0+\mathrm{F}(8))+\mathrm{E} 1 *(\mathrm{~F}(1)+\mathrm{F}(7))+\mathrm{E} 2 *(\mathrm{~F}(2)+\mathrm{F}(6))+$

C

$1 \mathrm{E3} *(\mathrm{~F}(3)+\mathrm{F}(5))+\mathrm{E} 4 * \mathrm{~F}(4)){ }^{*}$ STEP

$\mathrm{QADD}=\mathrm{QLEFT}+\mathrm{QRIGHT}$

DIFF $=$ QADD-QPREV

$\mathrm{C}$

AREA=AREA+DIFF

C

IF( ABS(QADD-EIGHT) .LE. RELERR*ABS(AREA) ) GOTO 99

C

$\operatorname{SIM}=\operatorname{STEP}^{*}\left(\mathrm{~F}(6)+\mathrm{F}(4)+4 .^{*}(\mathrm{~F}(7)+\mathrm{F}(5))+\mathrm{F}(8)+\mathrm{F}(6)\right) / 3$.

C

IF( ABS(QRIGHT-SIM) .LE. RELERR*ABS(AREA) ) GOTO 60

C

$\mathrm{LEV}=\mathrm{LEV}+1$

IF(LEV.GE.30)GOTO900

$\operatorname{FSAVE}(1, \mathrm{LEV})=\mathrm{F} 0$

$\mathrm{XSAVE}(1, \mathrm{LEV})=\mathrm{X} 0$

DO $52 \mathrm{I}=2,4$

FSAVE $(\mathrm{I}, \mathrm{LEV})=\mathrm{F}(\mathrm{I}-1)$

$\mathrm{XSAVE}(\mathrm{I}, \mathrm{LEV})=\mathrm{X}(\mathrm{I}-1)$

52 CONTINUE

QSAVE $($ LEV $)=$ QLEFT

C

$X 0=X(4)$
$F 0=F(4)$

C 


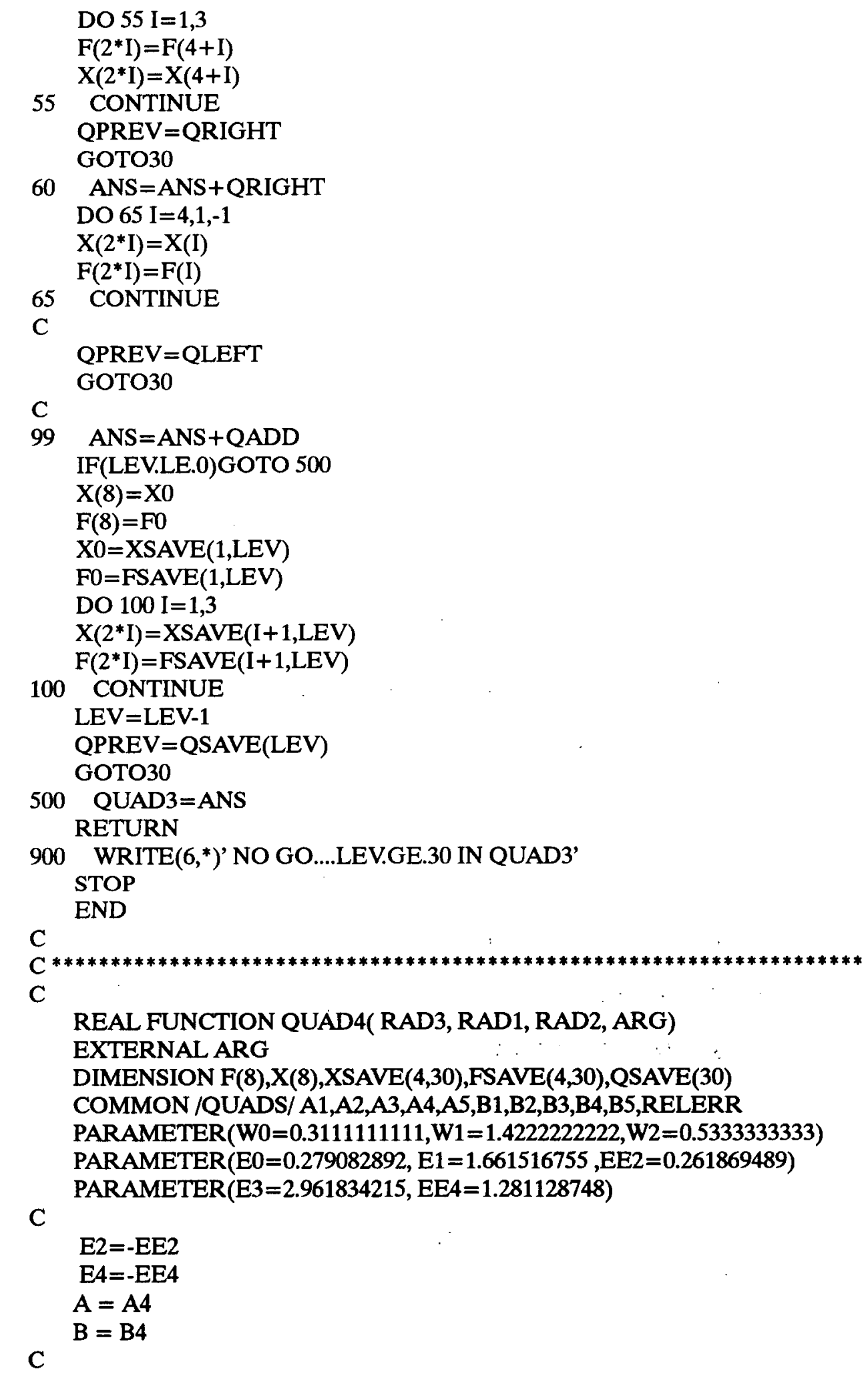


Appendix C

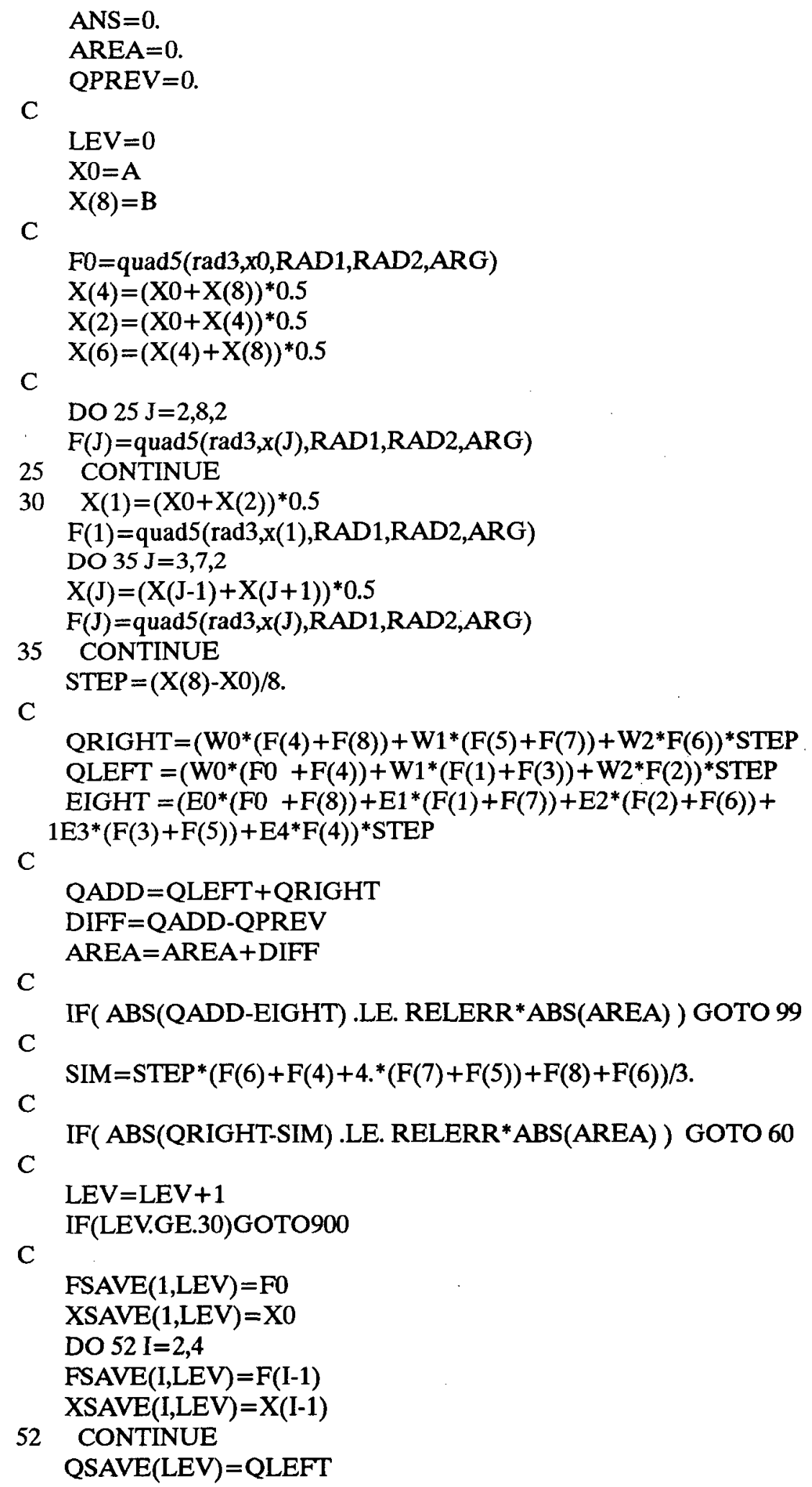


C

$\mathrm{X} 0=\mathrm{X}(4)$

$\mathrm{F} 0=\mathrm{F}(4)$

C

DO $55 \mathrm{I}=1,3$

$\mathrm{F}\left(2^{*} \mathrm{I}\right)=\mathrm{F}(4+\mathrm{I})$

$\mathrm{X}\left(2^{*} \mathrm{I}\right)=\mathrm{X}(4+\mathrm{I})$

55 CONTINUE

QPREV $=$ QRIGHT

GOTO30

60 ANS $=$ ANS + QRIGHT

DO $65 \mathrm{I}=4,1,-1$

$\mathrm{X}\left(2^{*} \mathrm{I}\right)=\mathrm{X}(\mathrm{I})$

$\mathrm{F}\left(2^{*} \mathrm{I}\right)=\mathrm{F}(\mathrm{I})$

65 CONTINUE

C

QPREV $=$ QLEFT

GOTO30

C

99 ANS $=$ ANS + QADD

IF(LEV.LE.0)GOTO 500

$\mathrm{X}(8)=\mathrm{X} 0$

$\mathrm{F}(8)=\mathrm{F} 0$

$\mathrm{X} 0=\mathrm{XSAVE}(1, \mathrm{LEV})$

F0 $=$ FSAVE $(1$, LEV $)$

DO $100 \mathrm{I}=1,3$

$\mathrm{X}\left(2^{*} \mathrm{I}\right)=\mathrm{XSAVE}(\mathrm{I}+1, \mathrm{LEV})$

$\mathrm{F}\left(2^{*} \mathrm{I}\right)=$ FSAVE $(\mathrm{I}+1, \mathrm{LEV})$

100 CONTINUE

$L E V=L E V-1$

QPREV $=$ QSAVE(LEV)

GOTO30

500 QUAD4 $=$ ANS

RETURN

900 WRITE $\left(6,{ }^{*}\right)$ ' NO GO....LEV.GE.30 IN QUAD4'

STOP

END

C

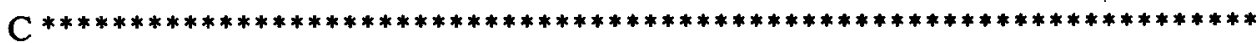

$\mathrm{C}$

REAL FUNCTION QUAD5( RAD3, RAD4, RAD1, RAD2, ARG)

EXTERNAL ARG

DIMENSION F(8),X(8),XSAVE(4,30),FSAVE(4,30),QSAVE(30)

COMMON /QUADS/ A1,A2,A3,A4,A5,B1,B2,B3,B4,B5,RELERR

PARAMETER $(W 0=0.3111111111, \mathrm{~W} 1=1.4222222222, \mathrm{~W} 2=0.5333333333$ )

PARAMETER(E0 $=0.279082892, \mathrm{E} 1=1.661516755, \mathrm{EE} 2=0.261869489)$

C

PARAMETER(E3 $=2.961834215, \mathrm{EE} 4=1.281128748)$

$\mathrm{E} 2=-\mathrm{EE} 2$ 
Appendix C

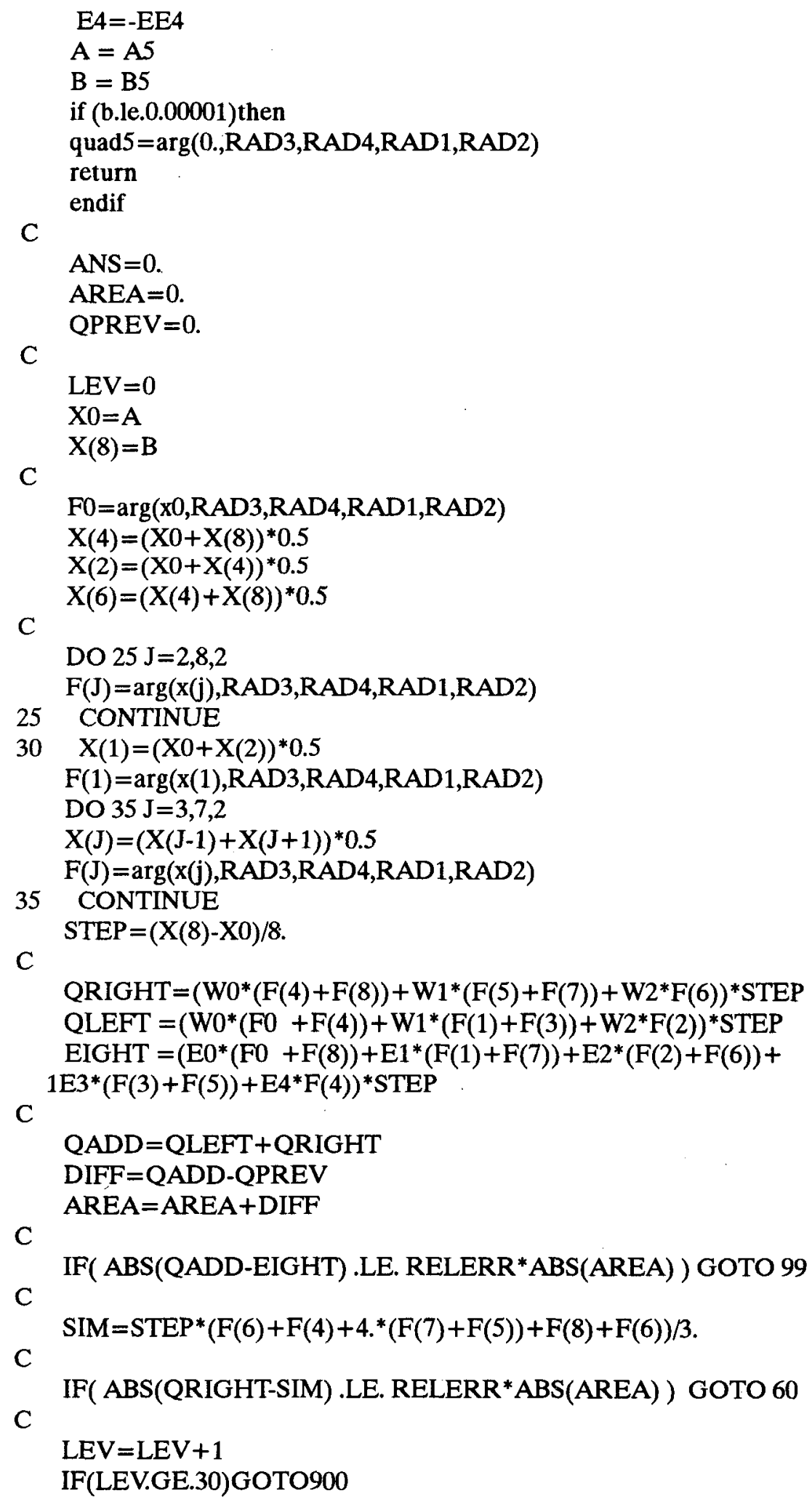


C

$\operatorname{FSAVE}(1, \mathrm{LEV})=\mathrm{F} 0$

$\mathrm{XSAVE}(1, \mathrm{LEV})=\mathrm{X} 0$

DO $52 \mathrm{I}=2,4$

FSAVE $(I, L E V)=F(I-1)$

XSAVE $(I, L E V)=X(I-1)$

52 CONTINUE

QSAVE $($ LEV $)=$ QLEFT

C

$\mathrm{X} 0=\mathrm{X}(4)$

$\mathrm{F} 0=\mathrm{F}(4)$

C

DO $55 \mathrm{I}=1,3$

$\mathrm{F}\left(2^{*} \mathrm{I}\right)=\mathrm{F}(4+\mathrm{I})$

$X(2 * I)=X(4+I)$

55 CONTINUE

QPREV $=$ QRIGHT

GOTO30

$60 \quad$ ANS $=$ ANS + QRIGHT

DO $65 \mathrm{I}=4,1,-1$

$\mathrm{X}\left(2^{*} \mathrm{I}\right)=\mathrm{X}(\mathrm{I})$

$\mathrm{F}\left(2^{*} \mathrm{I}\right)=\mathrm{F}(\mathrm{I})$

65 CONTINUE

C

QPREV $=$ QLEFT

GOTO30

C

99 ANS $=$ ANS + QADD

IF(LEV.LE.0)GOTO 500

$\mathrm{X}(8)=\mathrm{X} 0$

$\mathrm{F}(8)=\mathrm{F} 0$

$\mathrm{X} 0=\operatorname{XSAVE}(1, \mathrm{LEV})$

$\mathrm{F} 0=\mathrm{FSAVE}(1, \mathrm{LEV})$

DO $100 \mathrm{I}=1,3$

$\mathrm{X}(2 * \mathrm{I})=\mathrm{XSAVE}(\mathrm{I}+1, \mathrm{LEV})$

$\mathrm{F}\left(2^{*} \mathrm{I}\right)=\mathrm{FSAVE}(\mathrm{I}+1, \mathrm{LEV})$

100 CONTINUE

LEV $=$ LEV -1

QPREV $=$ QSAVE $($ LEV)

GOTO30

500 QUAD5 $=$ ANS

RETURN

900 WRITE(6,*)' NO GO....LEV.GE.30 IN QUAD5'

STOP

END

$\mathrm{C}$

$\mathrm{C} * * * * * * * * * * * * * * * * * * * * * * * * * * * * * * * * * * * * * * * * * * * * * * * * * * * * * * * * * * * * * * * * * * * * *)$

C

REAL FUNCTION ARG1(DRAD,SRAD,ALPHA,DUM1,DUM2) 
Appendix C

DIMENSION X(30),B(30),C(30),D(30), $\mathrm{FB}(30)$

COMMON X,FB,B,C,D,EAVE, $x 90$

COMMON/DENSE/ DENS,DENA,RMAX,DMAX,TA,TC,DC,XMAX,YMAX,PS

$\operatorname{dum} 1=0$.

dum2 $=0$.

$\mathrm{N}=30$

C Calculate the actual distance from source to point

depth $=$ dmax + tc + drad + ta

sarg $=$ depth*depth + srad*srad + ps*ps $-2 .{ }^{*}{ }^{*}$ srad $^{*}$ ps* ${ }^{*} \cos ($ alpha $)$

if(sarg.gt.-.0001.and.sarg.lt.0.)sarg $=0$.

rho $=$ sqrt (sarg)

if (rho.lt..000027)rho $=.000027$

$C$ Calculate the projected distance

sarg $=$ rho*rho - depth ${ }^{*}$ depth

if(sarg.gt.-.0001.and.sarg.1t.0.)sarg $=0$.

rhop $=$ sqrt(sarg)

if (rhop.lt..000027)rhop $=.000027$

rhoa $=0$.

xsors $=0$.

if(depth.gt.0.)xsors $=$ rho $^{*}$ drad/depth

$\mathrm{C}$ Calculate the distance traveled in the source

if(ps.le.rmax)then

C Exists through bottom

rhos $=$ xsors

else

if(srad.le.0.00001)then

$\operatorname{srad}=0.0$

$\cos b=1$.

else

$\operatorname{cosb}=\left(\right.$ ps*ps-srad ${ }^{*}$ srad-rhop*rhop $) /\left(2 .{ }^{*}\right.$ srad ${ }^{*}$ rhop $)$

endif

$C$ Look at source from side in plane of rho:

C xout is the projected distance from the center of the disk to the

$C$ location at which the path crosses the bottom of the disk

xout $=0$.

if(depth.gt.0.)xout $=$ drad*rhop/depth

C Look at source from the top: xproj is the projected distance

$C$ inside the source

sarg $=\operatorname{srad}^{*} \operatorname{srad}^{*} \cos b^{*} \cos b+\operatorname{rmax}^{*}{ }^{*} \max -$ srad $^{*}$ srad

if(sarg.le.0) sarg $=0$.

xproj=srad ${ }^{*} \operatorname{cosb}+$ sqrt(sarg)

if(xproj.le.0.000027) xproj $=0$.

if(xproj.lt.xout)then

C Exists through side of source

rhos $=$ xproj*rho/rhop

rhoa $=$ xsors - rhos

else

C Exists through bottom

rhos $=0$. 
if(drad.gt.0.)rhos $=$ drad $*$ rho/depth

endif

endif

C Calculate the actual distances in water, air, and cover material

rhoc $=0$.

if(depth.gt.0.)rhoc $=$ rho ${ }^{*}$ tc $/$ depth

rair $=0$.

if(depth.gt.0.)rair $=$ rho $*$ ta $/$ depth

rhow $=0$.

if (depth.gt.0.)rhow $=$ rho $*(\mathrm{dmax} / \mathrm{depth})$

path $=$ rhos ${ }^{*}$ dens $+($ rhoa + rair $) *$ dena + rhoc ${ }^{*} d c+$ rhow

if(path.le.0.000027)path $=0.000027$

SQIGL $=$ path $/$ X90

IF(SQIGL.GT.1.7999)THEN

$\mathrm{ARG} 1=0$.

ELSE

ARG1 $=$ SRAD * SEVAL (N,SQIGL,X,FB,B,C,D) / (RHO*RHO)

ENDIF

RETURN

END

$\mathrm{C}$

$C * * * * * * * * * * * * * * * * * * * * * * * * * * * * * * * * * * * * * * * * * * * * * * * * * * * * * * * * * * * * * * * * * * *)$

$\mathrm{C}$

REAL FUNCTION ARG2(PHI,SRAD,THETA,DUM1,DUM2)

DIMENSION X(30), B(30), C(30),D(30), $\mathrm{FB}(30)$

COMMON X,FB,B,C,D,EAVE, $\mathrm{x} 90$

COMMON/DENSE/ DENS,DENA,RMAX,DMAX,TA,TC,DC,XMAX,YMAX,PS

c

C

if(srad.eq.0.0)srad $=0.000027$

C Calculate the actual distance from source to point

$\mathrm{C}$

$\operatorname{dum} 1=0$

dum2 $=0$

$\mathrm{n}=\mathbf{3 0}$

dep1 $=$ dmax + rmax $+\operatorname{srad}^{*} \cos (p h i)+$ tc + ta

d1 $=\mathrm{dmax}+\mathrm{rmax}+\mathrm{tc}+\mathrm{ta}$

rsphi $=\operatorname{srad} * \sin (p h i)$

side $=\operatorname{dep} 1{ }^{*}$ dep $1+$ rsphi: ${ }^{*}$ rsphi + ps ${ }^{*}$ ps $-2 .{ }^{*}$ rsphi ${ }^{*}$ ps ${ }^{*} \cos ($ theta $)$

if(side.gt.. .0001 . and.side. 1 t. 0 .) side $=0$.

rho $=$ sqrt(side)

C Eliminate points that can not contribute

if (rho.lt..000027)rho $=.000027$

$\mathrm{C}$

C Calculate the distance above skin and skin covering

$\mathrm{C}$

$\mathrm{C}$

rhoask $=\operatorname{rho}^{*}\left(\operatorname{rmax}+\operatorname{srad}^{*} \cos (\mathrm{phi})\right) / \mathrm{dep} 1$

C Calculate the distance traveled in the source 
Appendix C

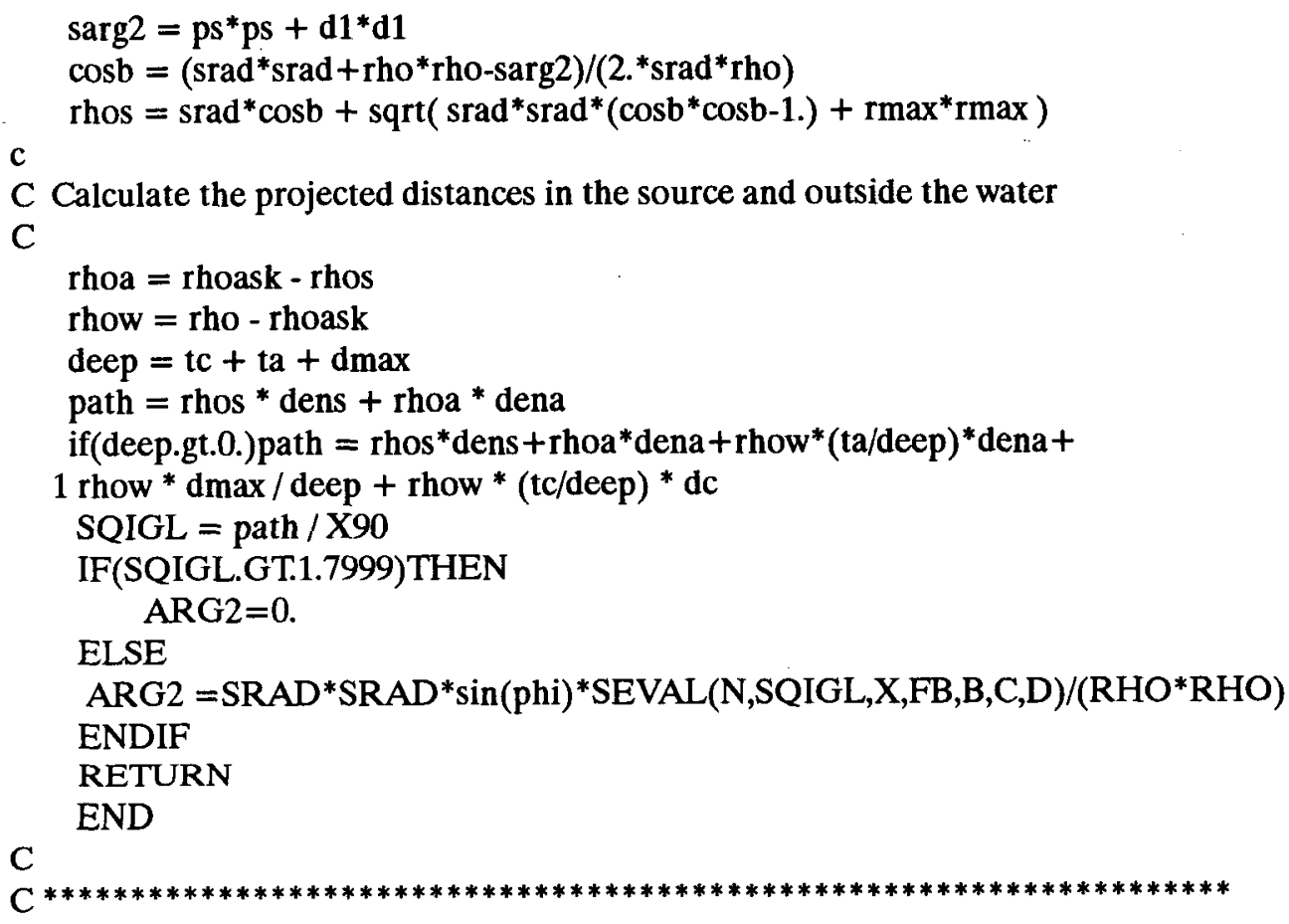

$\mathrm{C}$ Convert srad and alpha to cartesian coordinates

$\mathrm{xcar}=\operatorname{srad}^{*} \cos ($ alpha)

ycar $=\operatorname{srad}^{*} \sin ($ alpha $)$

$C$ Calculate the actual distance from source to point

xdis $=$ xrad - xcar

ydis $=$ yrad - ycar

$\mathrm{dep}=\mathrm{ta}+\mathrm{tc}+\mathrm{dmax}+\mathrm{zrad}$

sarg $=$ xdis*xdis +ydis*ydis + dep*dep

if(sarg.gt.-.0001.and.sarg.lt.0.)sarg $=0$.

rho $=$ sqrt(sarg)

if (rho.lt..000027)rho $=.000027$

rhoa $=0$.

C Calculate the actual distance above the source level dout $=0$. if(dep.gt.0.)dout $=$ rho ${ }^{*} \mathrm{zrad} / \mathrm{dep}$

C Check $x$ direction for exiting through side of source if(xcar.gt.xmax)then zunk $=\operatorname{dep} *(1 .+($ xrad-xmax $) /($ xcar-xrad $))$ if(zunk.gt.tc + dmax + ta)then

$C$ Exits through $x$-side of source rhoa $=$ dout $^{*}($ zunk-tc-dmax-ta $) /$ dep 
go to 1

endif

endif

C Check y direction for exiting through side of source

if(ycar.le.ymax)go to 1 zunk $=$ dep * $(1 .+($ yrad-ymax $) /($ ycar-yrad $))$

$C$ Exits through y-side of source if(zunk.gt.tc+dmax +ta)rhoa $=$ dout * $($ zunk-tc-dmax-ta) $/$ dep

$C$ Calculate the actual distances in the source, air, cover and water

1 rhos = dout - rhoa

rhow $=0$.

if(dep.gt.0.)rhow $=$ rho ${ }^{*}(\mathrm{dmax} / \mathrm{dep})$

rhoc $=0$.

if(dep.gt.0.)rhoc $=$ rho ${ }^{*}$ (tc/dep)

rair $=0$.

if(dep.gt.0.)rair $=$ rho $*$ (ta/dep)

path $=$ rhow + rhoc*dc $+\operatorname{rhos}^{*}$ dens $+(\text { rair }+ \text { rhoa })^{*}$ dena

if(path.1t.0.000027)path $=0.000027$

SQIGL $=$ path $/$ X90

IF(SQIGL.GT.1.7999)THEN

$$
A R G 3=0 .
$$

ELSE

ARG3 = SRAD * SEVAL $(\mathrm{N}, \mathrm{SQIGL}, \mathrm{X}, \mathrm{FB}, \mathrm{B}, \mathrm{C}, \mathrm{D}) /($ RHO $*$ RHO $)$

ENDIF

RETURN

END

$\mathrm{C}^{*}$

\section{SUBROUTINE SPLINE(M,X,Y,B,C,D)}

INTEGER N,M

C

REAL X(M), Y(M),B(M),C(M),D(M)

C THE COEFFICIENTS B(I), C(I), AND D(I), $I=1,2 . . . N$ ARE COMPUTED

C FOR A CUBIC INTERPOLATING SPLINE

C

$\mathrm{C} \mathrm{S}(\mathrm{X})=\mathrm{Y}(\mathrm{I})+\mathrm{B}(\mathrm{I}) *\left(\mathrm{X}-\mathrm{X}(\mathrm{I})+\mathrm{C}(\mathrm{I}) *(\mathrm{X}-\mathrm{X}(\mathrm{I}))^{* *} 2+\mathrm{D}(\mathrm{I})^{*}(\mathrm{X}-\mathrm{X}(\mathrm{I}))^{* * 3}\right.$

C

C FOR X(I).LE.X.LE.X(I+1)

$\mathrm{C}$

C INPUT..

C

C N= NUMBER OF DATA POINTS OF KNOTS (N.GE.2)

C $\mathrm{X}=$ THE ABSCISSAS OF THE KNOTS IN STRICTLY INCREASING ORDER

C $Y=$ THE ORDINATES OF THE KNOTS

$\mathrm{C}$

C OUTPUT..

$\mathrm{C}$

C B,C,D = ARRAYS OF SPLINE COEFFICIENTS AS DEFINED ABOVE 


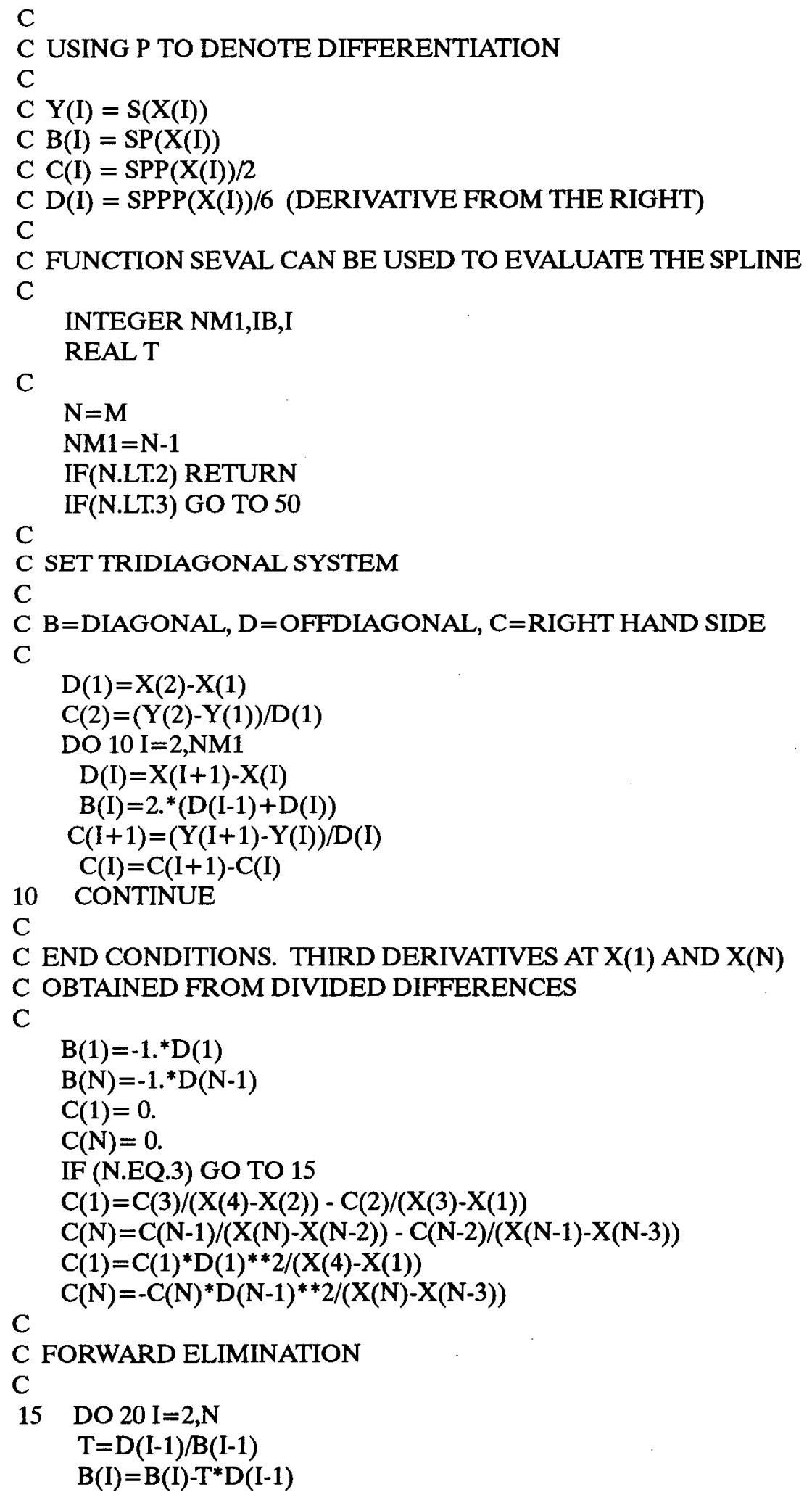




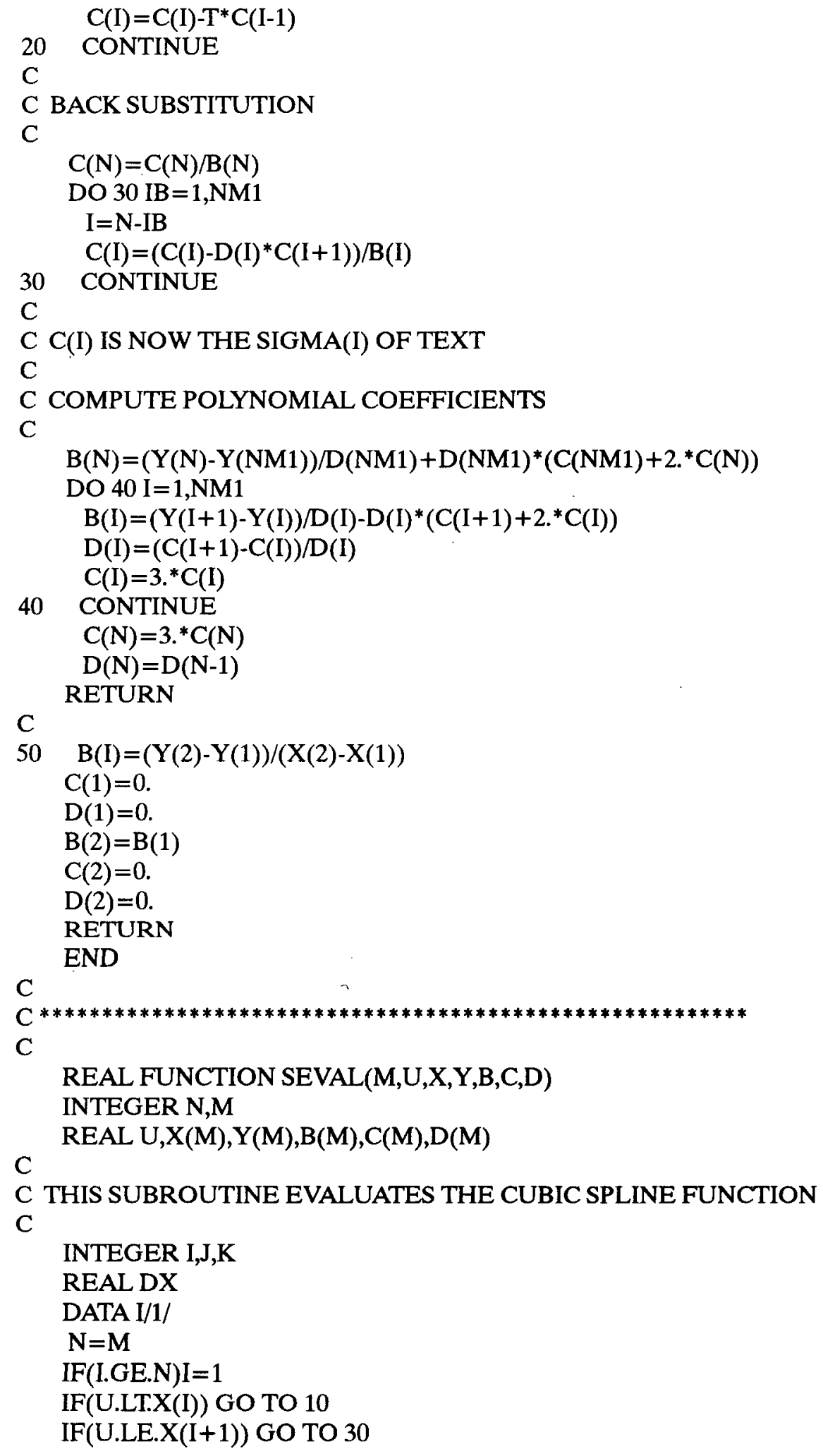


Appendix C

C

C BINARY SEARCH

C

$10 \quad \mathrm{I}=1$

$\mathrm{J}=\mathrm{N}+1$

$20 \mathrm{~K}=(\mathrm{I}+\mathrm{J}) / 2$

$\operatorname{IF}(U . L T . X(K)) J=K$

IF(U.GE.X(K)) $\mathrm{I}=\mathrm{K}$

IF(J.GT.I+1) GO TO 20

$C$ EVALUATE SPLINE

$30 \quad \mathrm{DX}=\mathrm{U}-\mathrm{X}(\mathrm{I})$

SEVAL $=\mathrm{Y}(\mathrm{I})+\mathrm{DX}^{*}\left(\mathrm{~B}(\mathrm{I})+\mathrm{DX}^{*}\left(\mathrm{C}(\mathrm{I})+\mathrm{DX} \mathrm{X}^{*} \mathrm{D}(\mathrm{I})\right)\right)$

RETURN

END 


\section{APPENDIX D}

TEXT OF THE USER INTERFACE MODULES 


\section{APPENDIX D}

TEXT OF THE USER INTERFACE MODULES

\section{PROGRAM FILE VARMAIN.C}

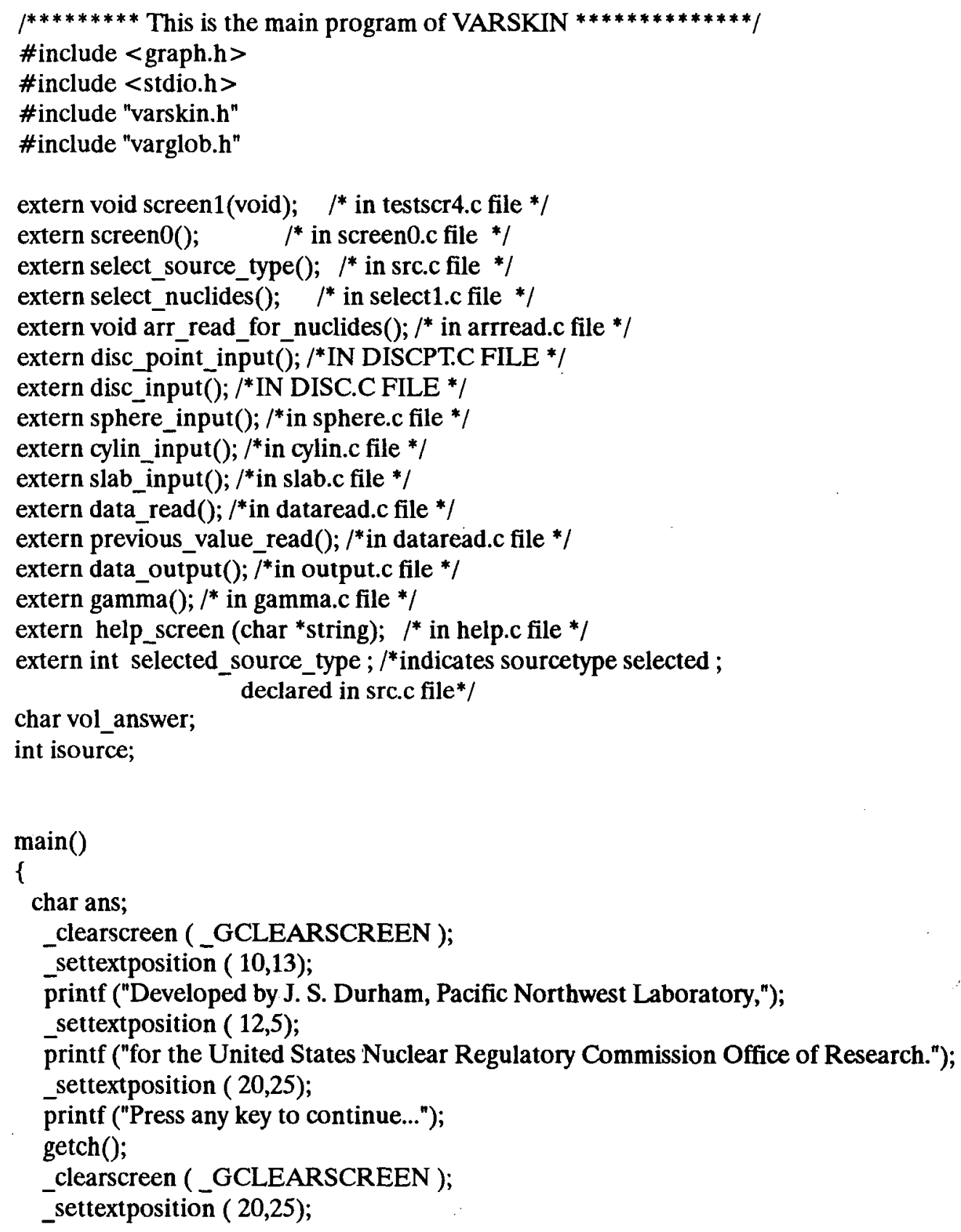




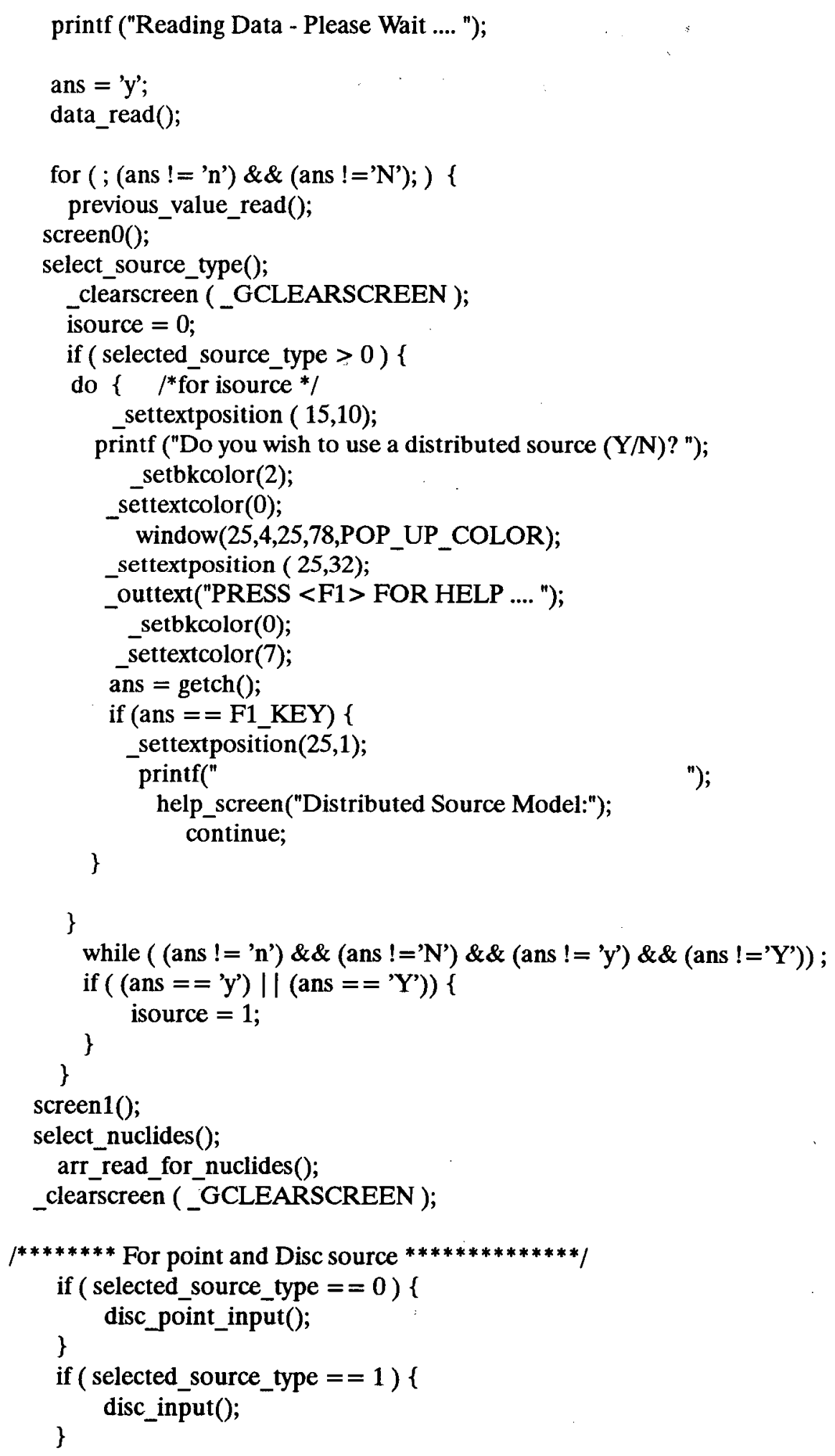




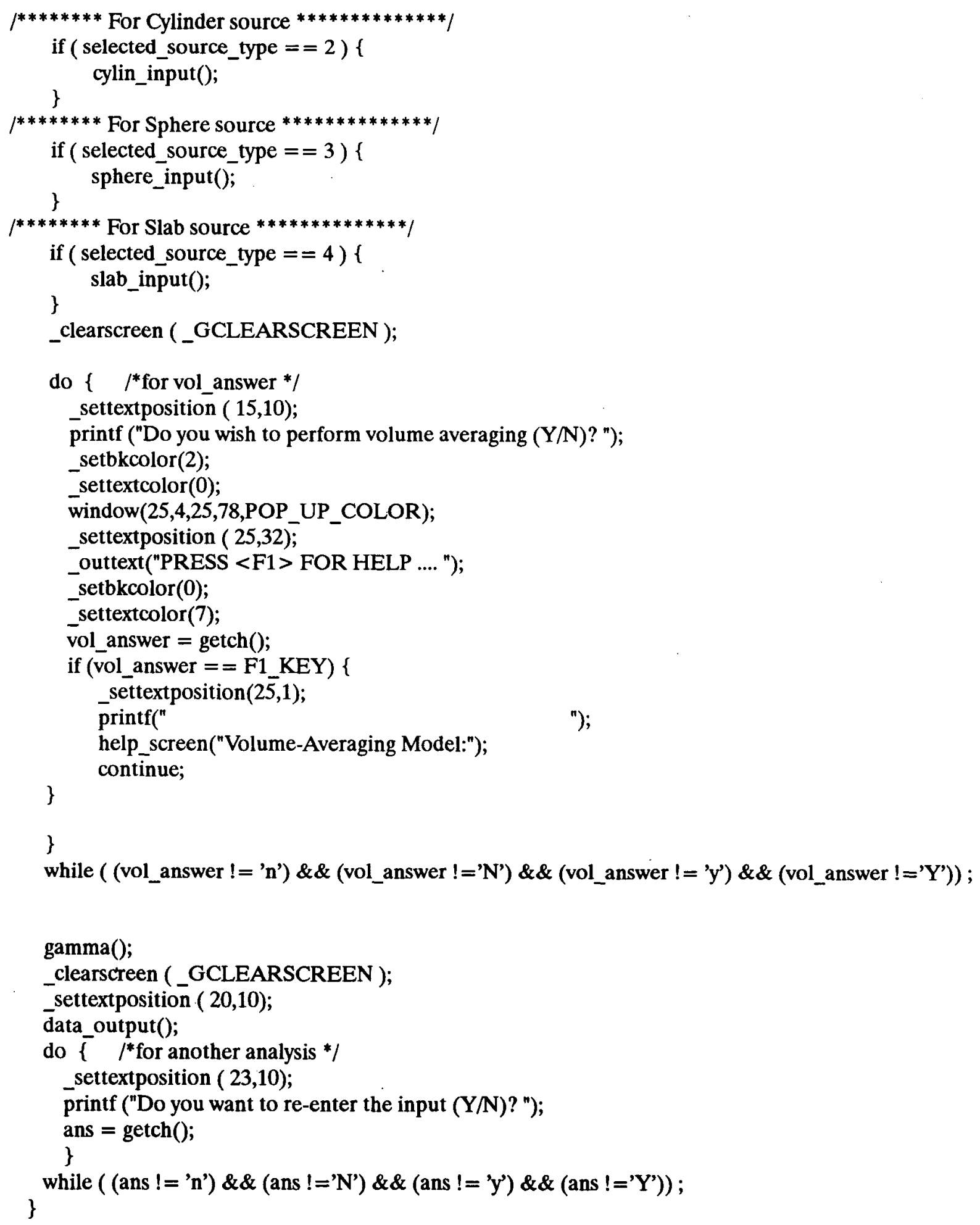


Appendix D

$\left.\right|^{* * * * * * * * * *}$ End of main program of VARSKIN $* * * * * * * * * * * * /$ 


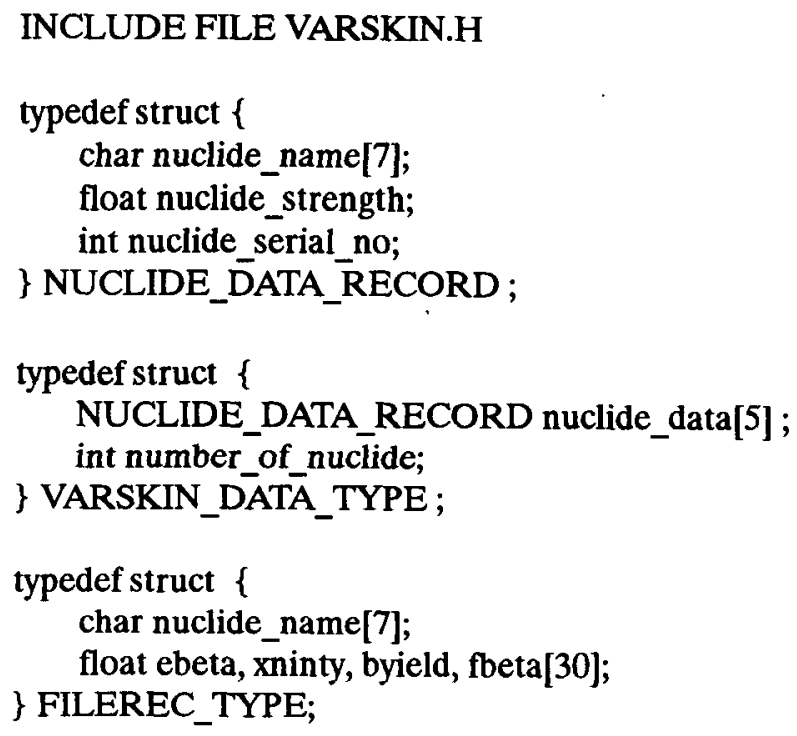


Appendix D

INCLUDE FILE VARGLOB.H

\#define BASE 0xb8000000L

$\begin{array}{lr}\text { \#define LENGTH_OF_SRC 8 } & / * \text { length of each SOURCE item * } / \\ \text { \#define LENGTH_OF_NUCLIDE } 7 & / * \text { length of each NUCLIDE item */ }\end{array}$

I* video address */

\#define LEN 8 $/^{*}$ length of each item */

\#define HIGH 0x71 /* intensified attribute for item highlight*/

\#define BK_COLOR 0x17 /*reverse backgroun color for nuclides */

\#define POP_UP_COLOR 0x27 /*pop up_window color */

\#define WINDOW

\#define ESCAPE_KEY 27

\#define ENTER_KKEY 13

\#define MAX_NUMBER_OF_SELECTION 5

\#define DOWN_KEY 80

\#define UP_KEY 72

\#define LEFTT_KEY 75

\#define RIGHT_KEY 77

\#define F1_KEY 59

\#define PGDN_KEY 81

\#define PGUP_KEY 73

\#define SPACE $0 \times 20$

\#define BACKSPACE 8

\#define DEL_KEY 83 


\section{INCLUDE FILE NUCLIDE.H}

\section{\#define ROW1 3 \\ /* Row position of 1 st nuclide */}

\#define COL1 5

\#define NUCLIDE_PER_LINE 6

\#define NUMBER_OF_R̄OW 14

\#define COL_DISTANCE $13 / /$ distance between adjacent nuclides */

\#define ROW_DISTANCE $1 /{ }^{*}$ distance between rows of nuclides */ 
Appendix D

INCLUDE FILE SRCTYPE.H

\#define ROW1 $8 \quad I^{*}$ Row position of 1st nuclide *

\#define COL1 $45 \quad / *$ Column position of 1st nuclide */

\#define SOURCE_PER_LINE 1

\#define NUMBER_OF_ROW 5

\#define COL_DISTANCE $0 \quad / *$ distance between adjacent source_type */

\#define ROW_DISTANCE $2 \quad / *$ distance between rows of source_type */ 


\section{PROGRAM FILE SRC.C}

\#include <stdlib.h>

\#include <graph.h>

\#include <stdio.h $>$

\#include "varskin.h"

\#include "varglob.h"

\#include "srctype.h"

extern char *source_type $] ; \quad / *$ in select $0 . c$ file */

extern get_string(char *string, int string_size, int text_row , int text_col); $/{ }^{*}$ in select1.c file*/

extern save_back_ground( int top_row,int left_col, int bottom_row, int right_col);

extern restore_back_ground (int top_row,int left_col, int bottom_row, int right_col);

extern memory_copy ( char far *source, char far * dest, int bytes_in_a_line );

extern high_light (int row, int col, int length);

extern normal_light ( int row, int col, int length);

extern help_screen (char *string); $/{ }^{*}$ in help.c file * $/$

extern window( int window_row1, int window_col1, int window_row2, int window_col2, char attr);

$/ *$ in select0.c file ${ }^{*} /$

int selected_source_type; /*indicates sourcetype selected */

$/ * * * * * * * * * * *$ This function selects an item from a list $* * * * * * * * * * * /$

select_source_type()

\{

const int string_size $=8$;

int row, col, size;

char c;

char skin_string[8];

char diameter_string[8];

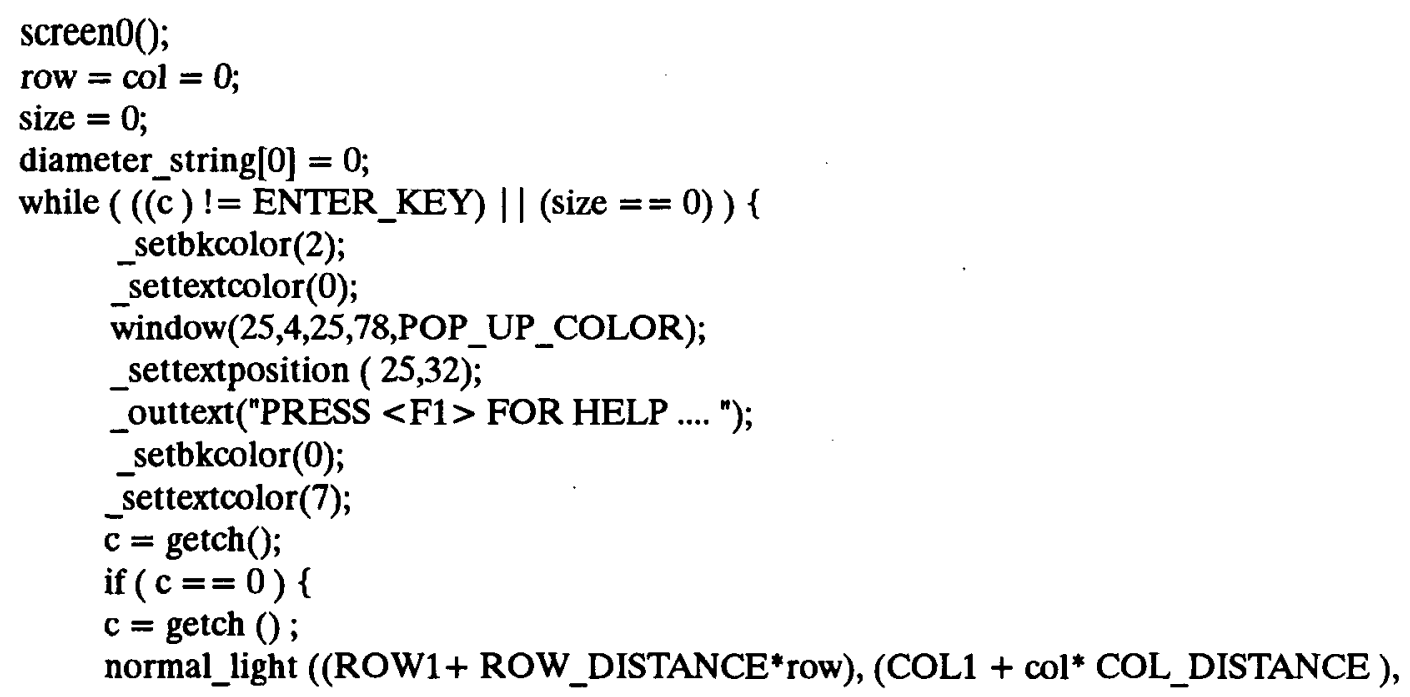


Appendix D

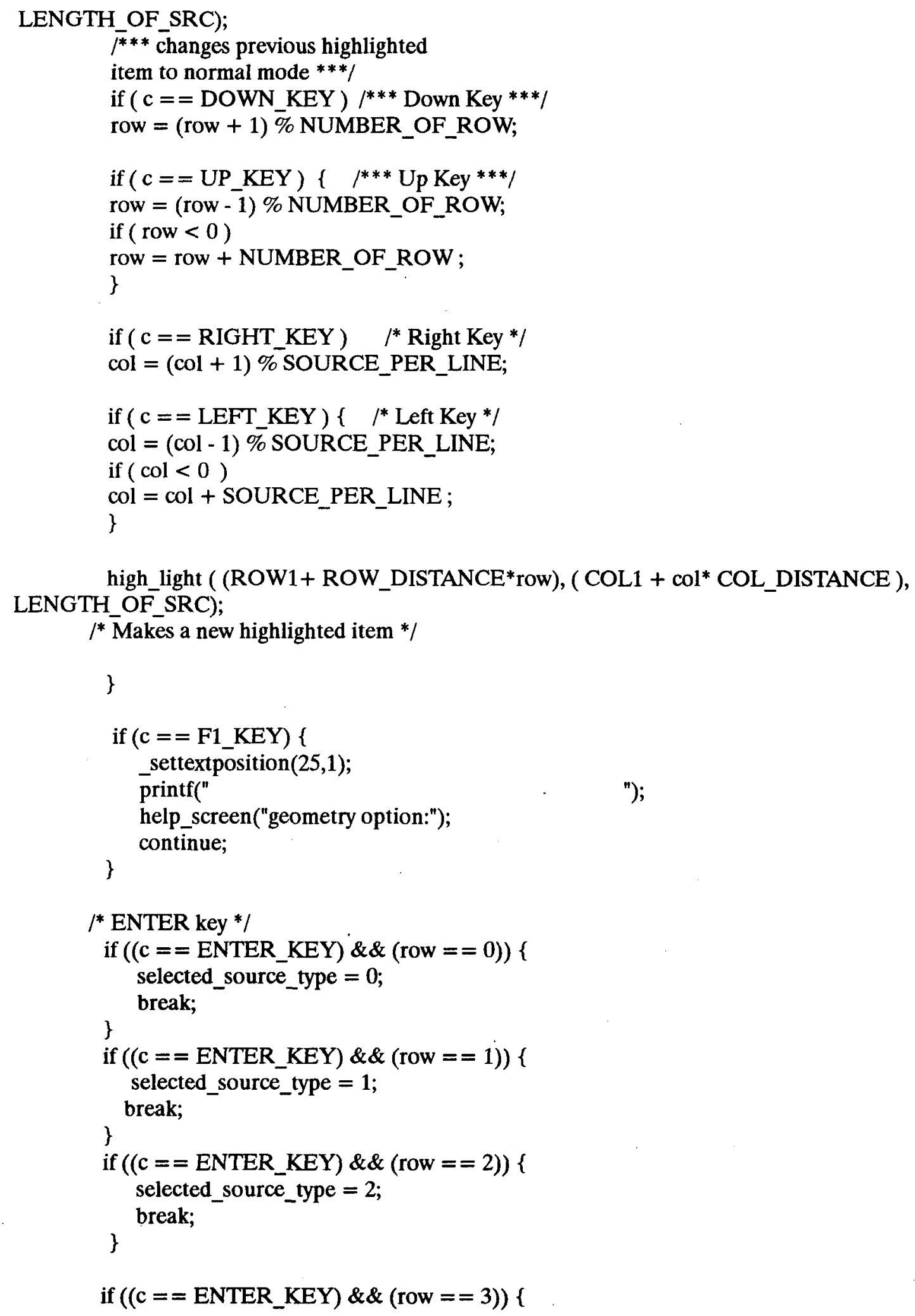




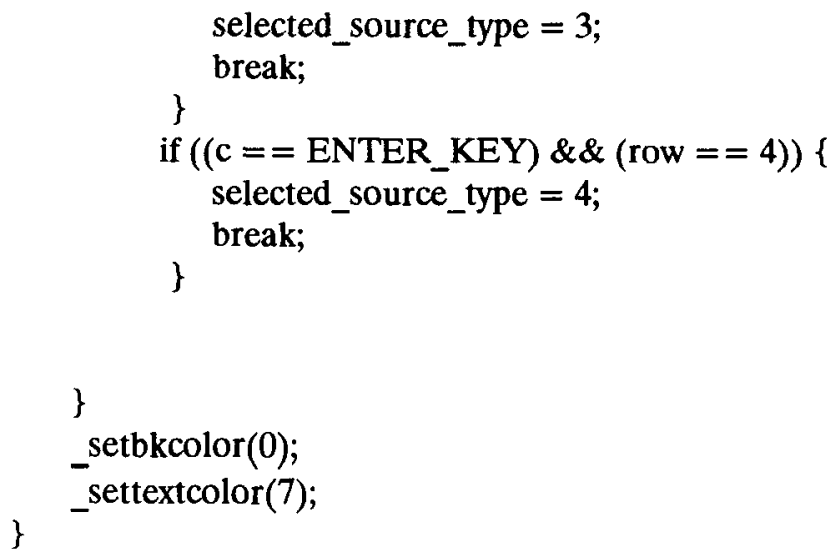


Appendix D

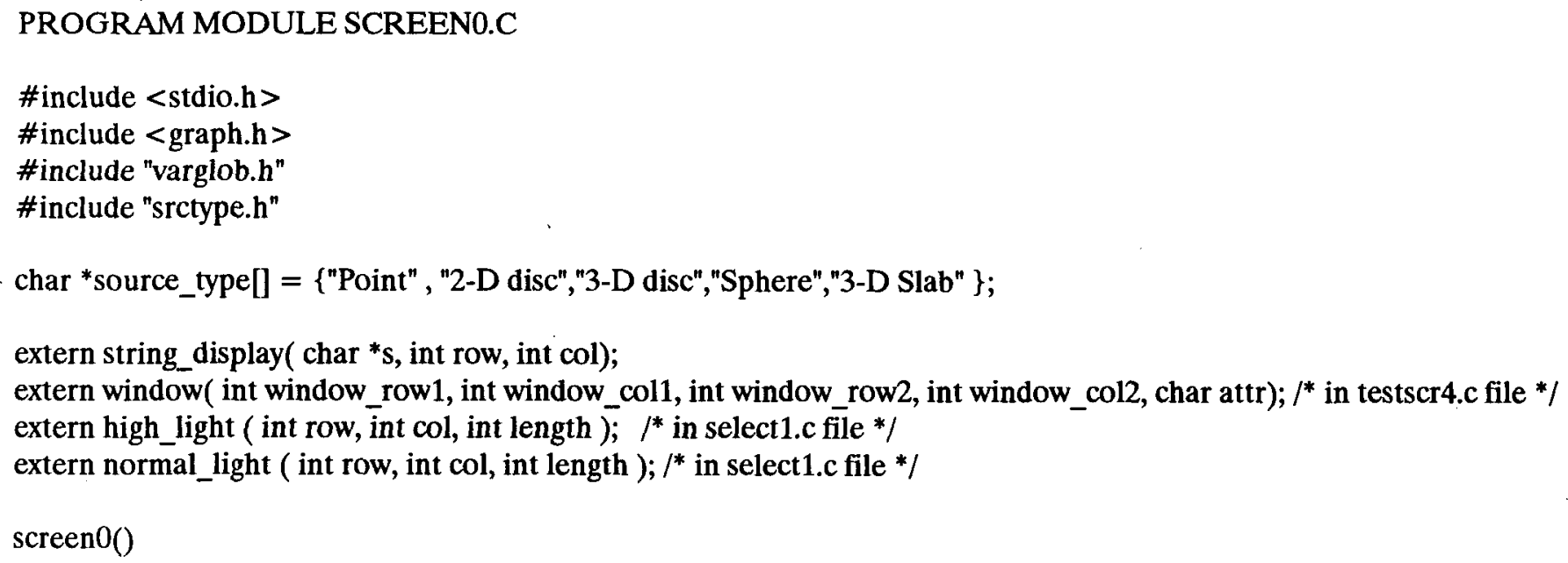




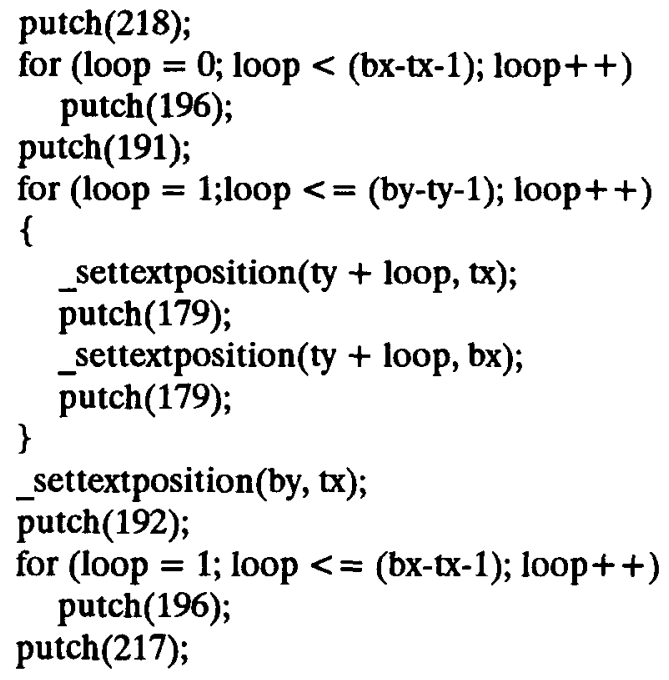


Appendix D

\section{PROGRAM MODULE TESTSCR4.C}

\#include < stdio.h $>$

\#include < graph.h >

\#include "varglob.h"

\#include "nuclide.h"

extern high_light ( int row, int col, int length ); $l^{*}$ in select1.c file */

extern normal_light ( int row, int col, int length ); ${ }^{*}$ in select1.c file */

extern char nuclides_all[100][8];

extern int total_nuclides;

void screen1(void)

/****** This program makes a Pull-Down Menu to select an item ******/

\{

int col_count, row_count, row, col ;

_clearscreen(_GCLEARSCREEN);

_settextposition( 1, (80 - strlen("Program VARSKIN Mod 2"))/2);

printf("Program VARSKIN Mod 2");

row $=$ ROW 1

$\mathrm{col}=\mathrm{COL1}$

window (3,4,17,78,BK_COLOR); /* makes a highlighted window */

for ( row_count $=0$; row_count $<$ NUMBER_OF_ROW; row_count ++ ) \{ for $($ col_count $=0$; col_count $<$ NUCLIDE_PER_LINE; col_count ++ ) \{ string_display (nuclides_all[row_count*NUCLIDE_PER_LINE + col_count], row , col );

high_light (ROW1, COL1, LENGTH_OF_NUCLIDE); /* highlights the first item */

\}

$1 * * * * * * * * * * * * * * * * * * * *$ End of screen 1()

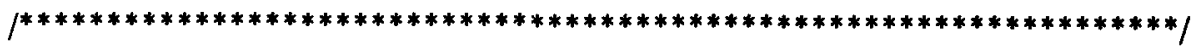

/*** Displays a string at a particular position $* * * /$

string_display( $s$, row, col)

char *s;

int row, col ;

\{

char far *pt;

register char c;

NUREG/CR-5873 
int width;

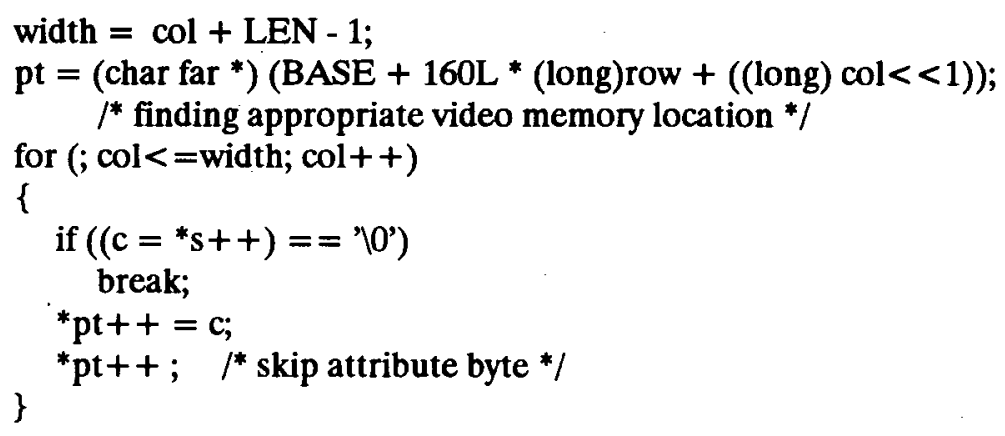




\section{Appendix D}

\section{PROGRAM MODULE DATAREAD.C}

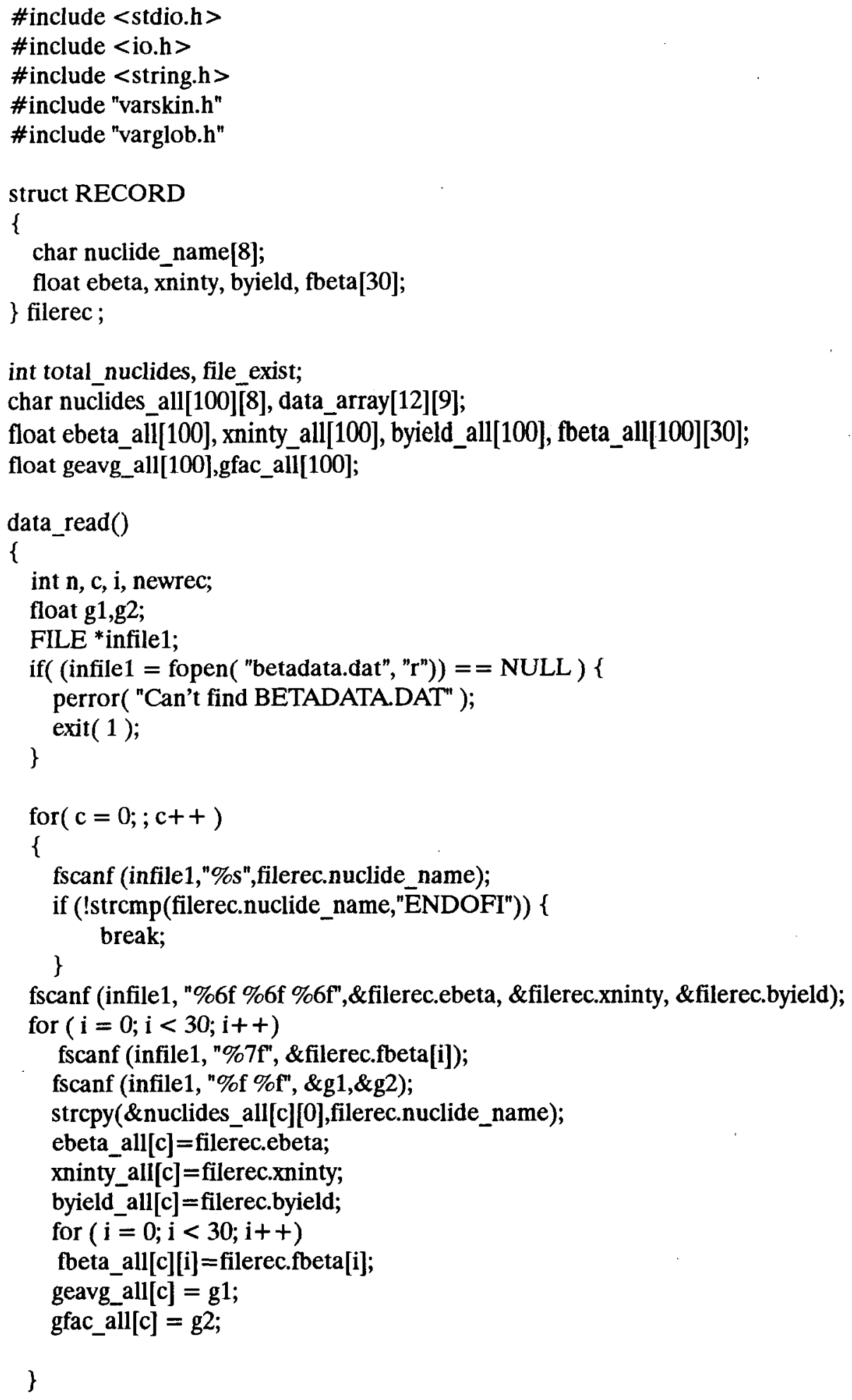




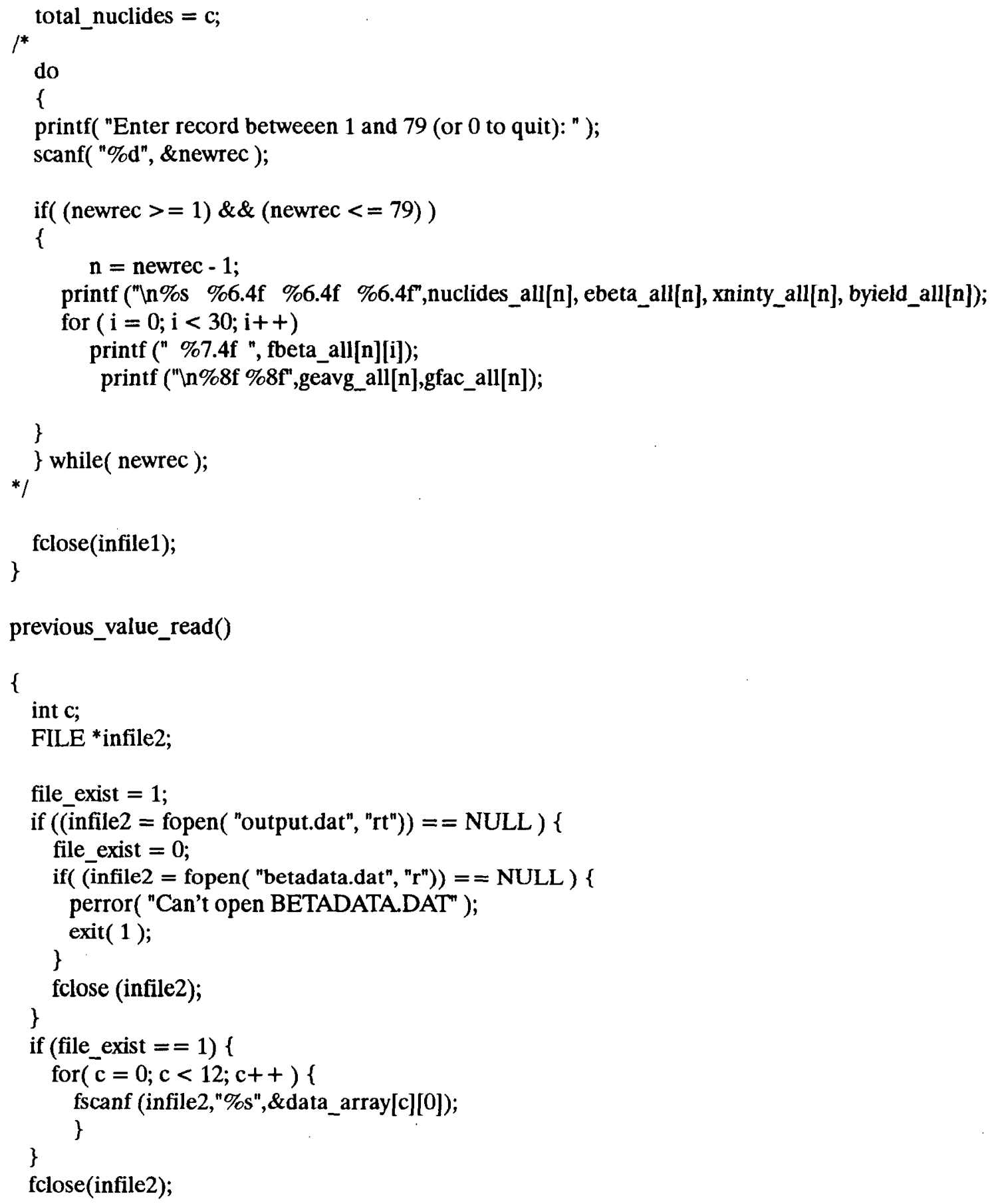


Appendix D

PROGRAM MODULE ARRREAD.C

\#include < graph.h $>$

\#include < stdio.h $>$

\#include "varskin.h"

extern VARSKIN_DATA_TYPE varskin_data; $\quad / *$ definition in varskin.h file */ extern char nuclides_all[100][8];

extern float ebeta_all[100], xninty_all[100], byield_all[100], fbeta_all[100][30];

extern float geavg_all[100], gfac_all[100];

float ebeta[6], xninty[6], byield[6], fbeta[6][30];

float geavg[6], gfac[6];

$/ * * * * * * * *$ This function reads other nuclide information from file $* * * * * * /$

void arr_read_for_nuclides()

\{

int $c, i$, newrec;

for $(i=0 ; i<$ varskin_data.number_of_nuclide $; i++)\{$

newrec $=$ varskin_data.nuclide_data[i].nuclide_serial_no ;

ebeta[i] = ebeta_all[newrec];

xninty[i] = xninty_all[newrec];

byield[i] = byield_all[newrec];

geavg[i] = geavg_all[newrec];

gfac[i] = gfac_all[newrec];

for $(c=0 ; c<30 ; c++)$

\}

fbeta[i][c] = fbeta_all[newrec] $[c]$;

\}

$/^{* * * * * * * * * * * *}$ End of fileread_for_nuclides() 
PROGRAM MODULE SELECT1.C

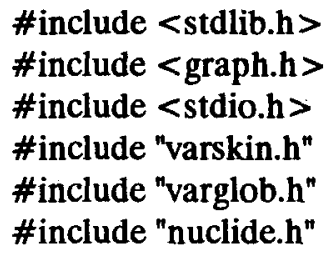

VARSKIN_DATA_TYPE varskin_data; $/{ }^{*}$ definition in varskin.h file */

extern int total_nuclides;

extern char nuclides_all[100][8]; $\quad I^{*}$ in testscr4.c file $* /$

extern window( int window_row1, int window_col1, int window_row2, int window_col2, char attr);

$l^{*}$ in testscr4.c file */

extern msg_screen (char *str); /*in cylin.c file */

extern int isource; /*indicates distributed source or not ; in varmain.c file*/

extern int selected_source_type; /*indicates sourcetype selected */

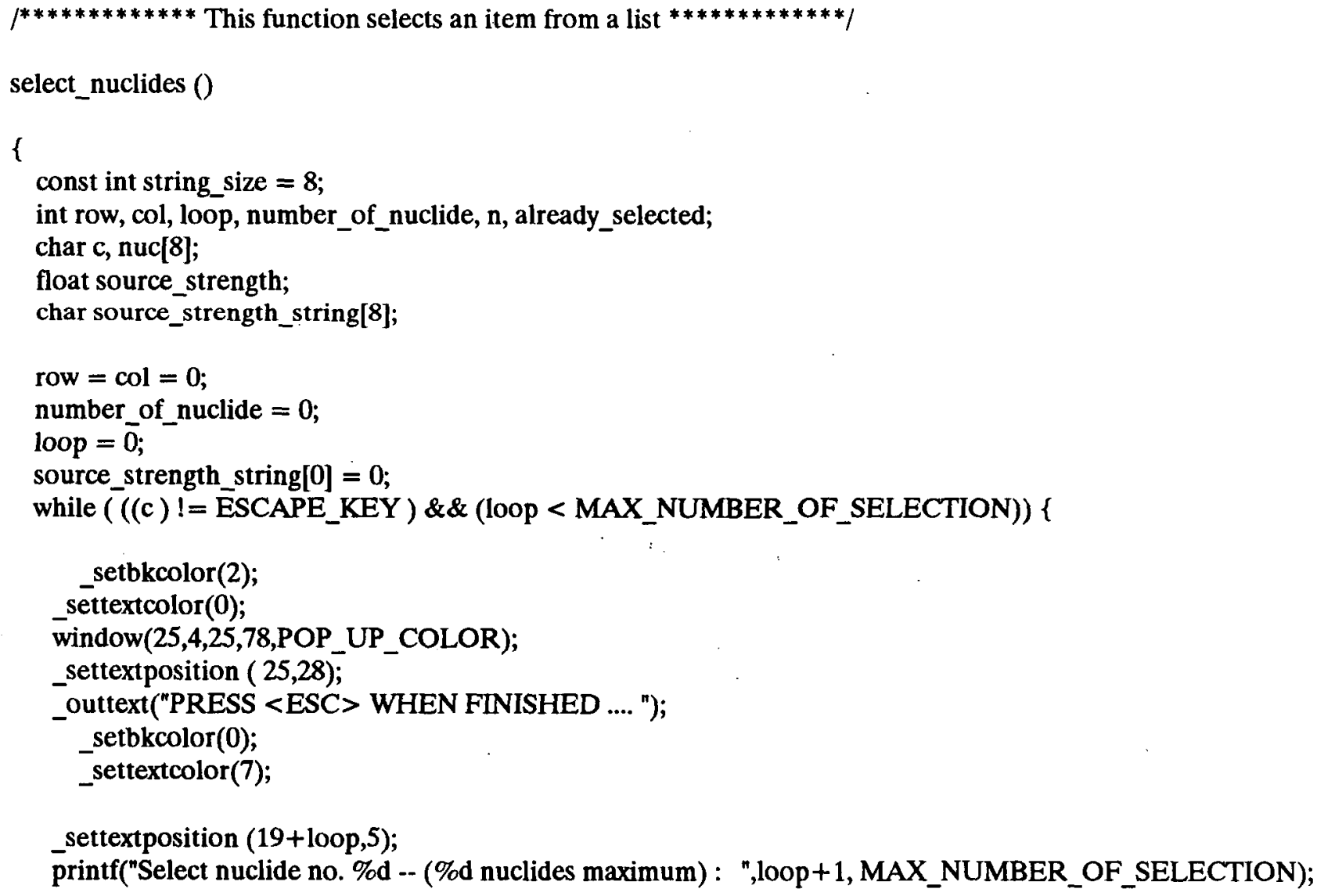


Appendix D

while $(((c=$ getch ()$) !=$ ESCAPE_KEY $) \|($ number_of_nuclide $==0))\{$

if $((\mathrm{c}==$ ESCAPE_KEY $) \& \&$ (number_of_nuclide $==0))\{$

putchar(BELL);

msg_screen ("Select atleast one nuclide ");

_settextposition $(19,54)$;

$$
\text { if }(c==0)\{
$$

$\mathrm{c}=\operatorname{getch}()$;

normal_light ((ROW1+ROW_DISTANCE*row), (COL1 + col* COL_DISTANCE

),LENGTH_OF_NUCLIDE);

$/^{* * *}$ changes previous highlighted

item to normal mode $* * *$ /

if $(\mathrm{c}==$ DOWN_KEY) $/ * * *$ Down Key ***/

row $=($ row $+\overline{1}) \%$ NUMBER_OF_ROW;

if $(\mathrm{c}==$ UP_KEY $)\{\quad / * *$ Up Key $* * * /$

row $=($ row - 1$) \%$ NUMBER_OF_ROW;

if $($ row $<0)$

\}

row $=$ row + NUMBER_OF_ROW;

if $(\mathrm{c}==\mathrm{RIGHT}$ KEY ) $\quad / *$ Right Key $* /$

$\mathrm{col}=(\mathrm{col}+1) \%$ NUCLIDE_PER_LINE;

if $(\mathrm{c}==$ LEFT_KEY $)\{\quad / *$ Left Key $* /$

$\mathrm{col}=(\mathrm{col}-1) \%$ NUCLIDE_PER_LINE;

if $(\mathrm{col}<0)$

\}

$\mathrm{col}=\mathrm{col}+$ NUCLIDE_PER_LINE;

high_light ( (ROW1+ ROW_DISTANCE*row), (COL1 + col* COL_DISTANCE),

\section{LENGTH OF NUCLIDE);}

$l^{*}$ Makes a new highlighted item */

\}

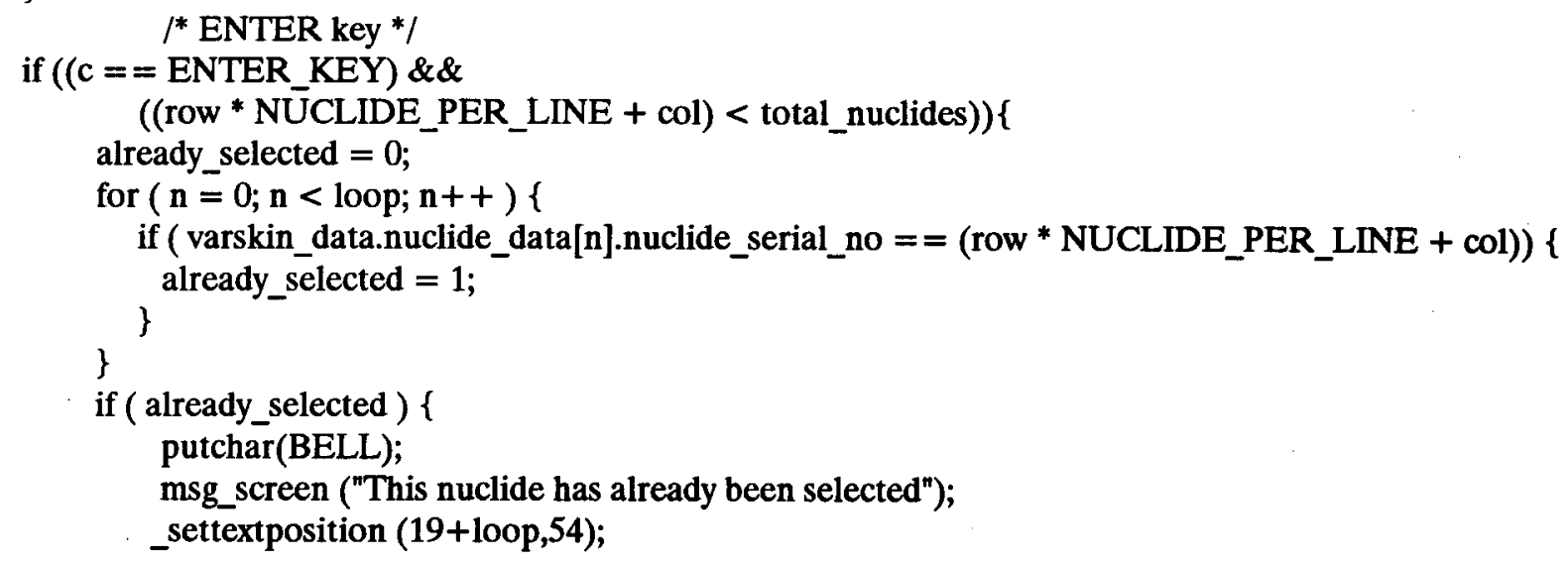


\}

else \{

setbkcolor(2);

settextcolor(0);

window $(25,4,25,78$, POP_UP_COLOR);

_settextposition (25,23);

_outtext("PRESS <ESC> TO SKIP THIS NUCLIDE .... ");

save_back_ground $(11,21,18,71) ; / *$ saves the window $(11,21)$ to $(18,71) * /$

window(11,21,18,71,POP_UP_COLOR);

_settextposition (12,22);

_outtext("Enter Source strength for ");

_outtext (nuclides_all[row * NUCLIDE_PER_LINE + col]);

settextposition (12,57);

window $(12,57,12,64,0 \times 07)$;

$/ *$ printf(" "); */ /* makes input number area */

_settextposition (14,22);

if (isource $==0$ ) \{

_outtext ("Enter source strength in microcuries");

$\overline{\}}$

if (isource $==1$ ) \{

if ( selected_source_type $==1$ ) \{

\}

_outtext ("Enter source strength in microCi/ $\mathrm{cm}^{\wedge} 2^{n}$ );

if ( selected_source_type $>1$ ) \{

\}

_outtext ("Enter source strength in microCi/(cm^^3");

\}

get_string ( source_strength_string, string_size, 12, 57);

$1 *$ Reads string with valid digits */

if (strlen (source_strength_string) $==0)\left\{I^{*}\right.$ Escaping from inputting strength *

restore_back_ground $(11,21,18,71) ; I^{*}$ retrieves window $(11,21)$ to $(18,71) * /$

window(25,4,25,78,POP_UP_COLOR);

_settextposition $(25,23)$;

_outtext("PRESS < ESC> WHEN FINISHED ...." );

_settextposition $(19+$ loop,54);

continue ;

\}

_settextposition (19+loop,54);

printf ("\%s",nuclides_all[row * NUCLIDE_PER_LINE + col]);

source_strength $=$ atof(source_strength_string); $/^{*}$ converts ascii to float */

_settextposition $(19+$ loop,65);

printf("\%f",source_strength);

strcpy (varskin_data.nuclide_data[loop].nuclide_name, nuclides_all [row * NUCLIDE_PER_LINE + col] ); varskin_data.nuclide_data[loop].nuclide_strength = source_strength;

varskin_data.nuclide_data[loop].nuclide_serial_no $=$ row * NUCLIDE_PER_LINE + col ;

number_of_nuclide $=100 \mathrm{p}+1$;

restore_back_ground $(11,21,18,71) ; l^{*}$ retrieves window $(11,21)$ to $(18,71) * /$ 
Appendix D

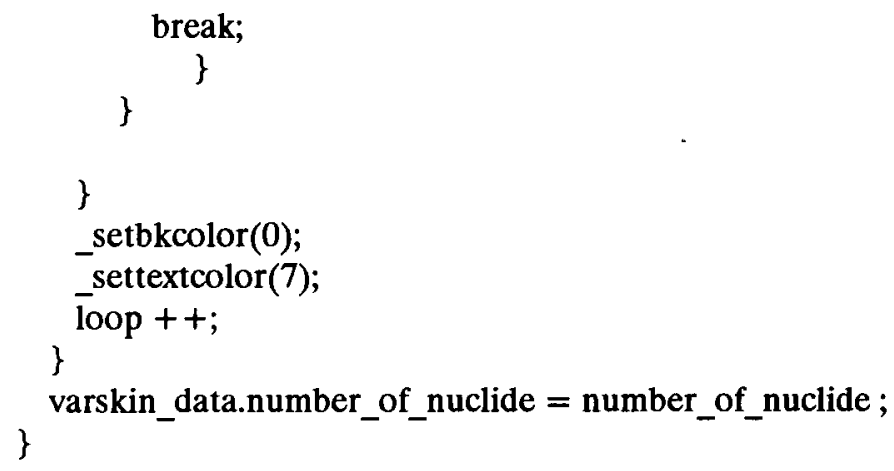


/* Filtering out invalid characters */

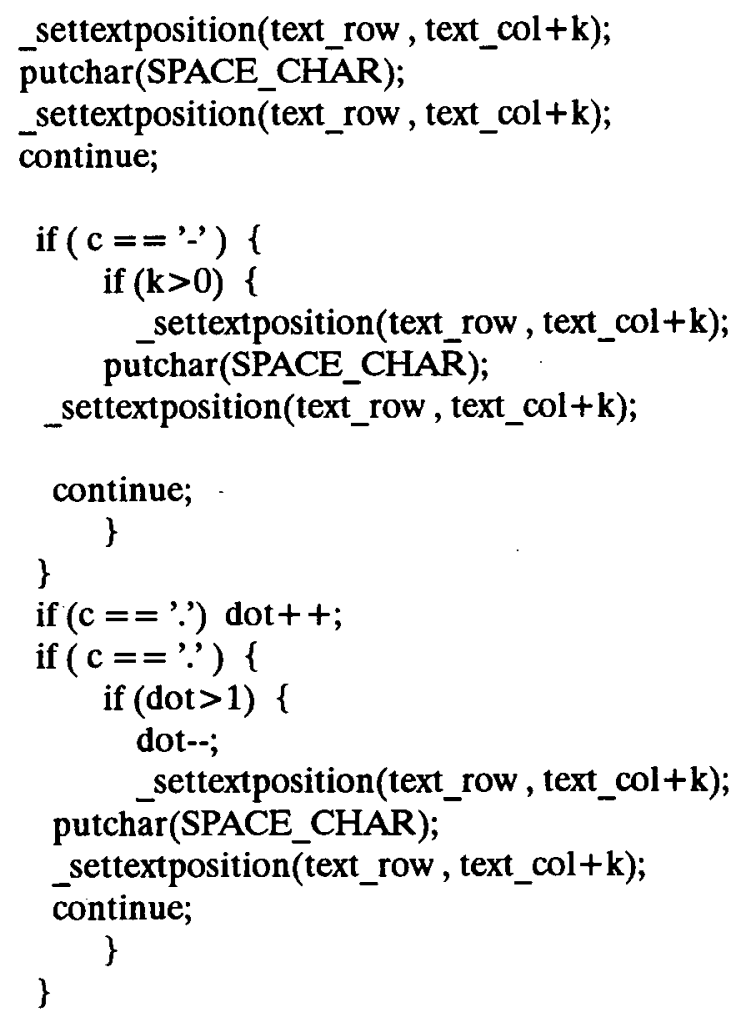


Appendix D

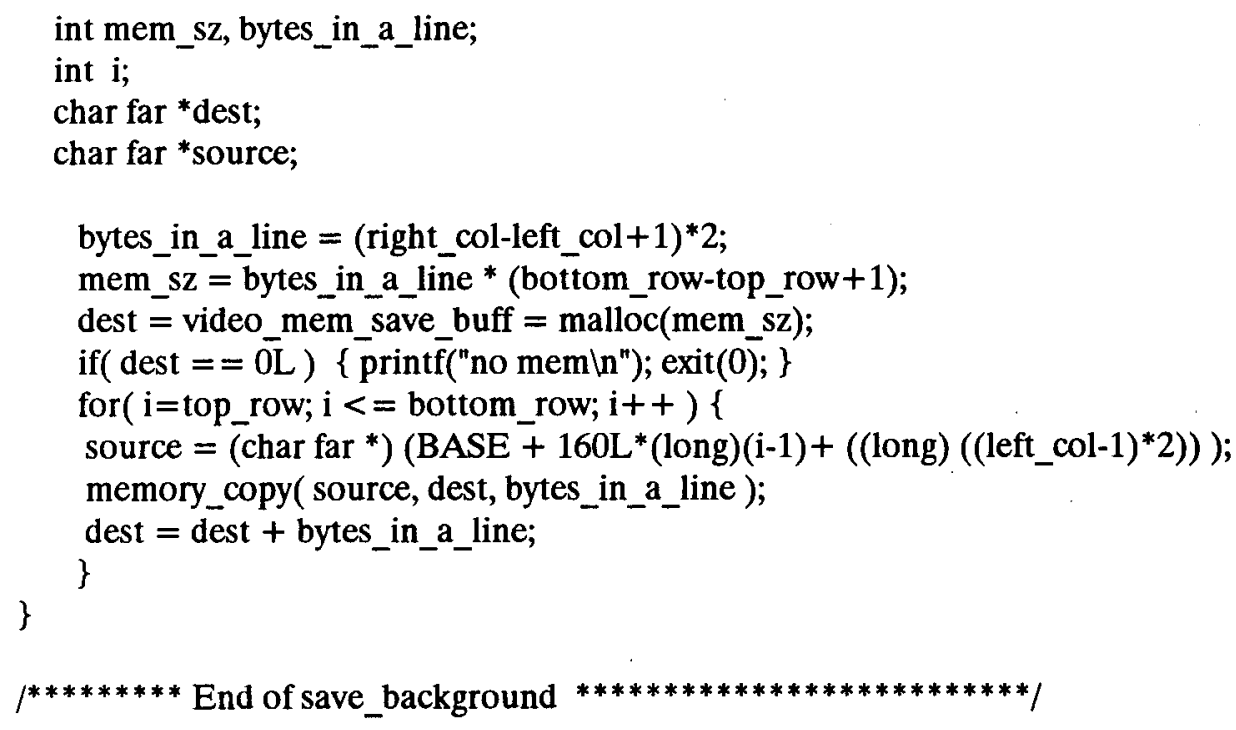




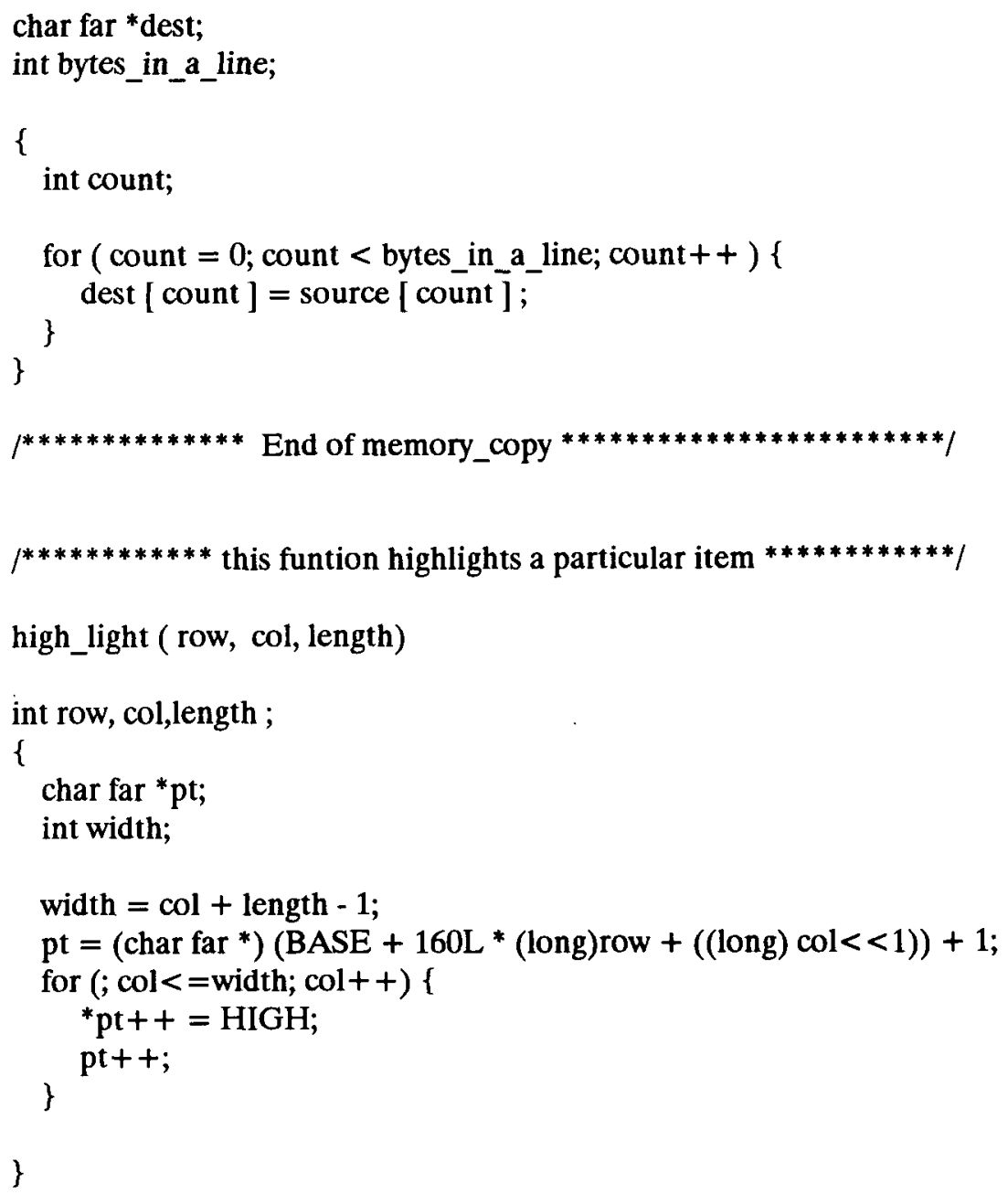


Appendix D

\}

$/{ }^{* * * * * * * * * * * * * * * * * *}$ End of normal_light $* * * * * * * * * * * * * * * * * * * * * * * /$ 


\section{PROGRAM MODULE DISCPT.C}

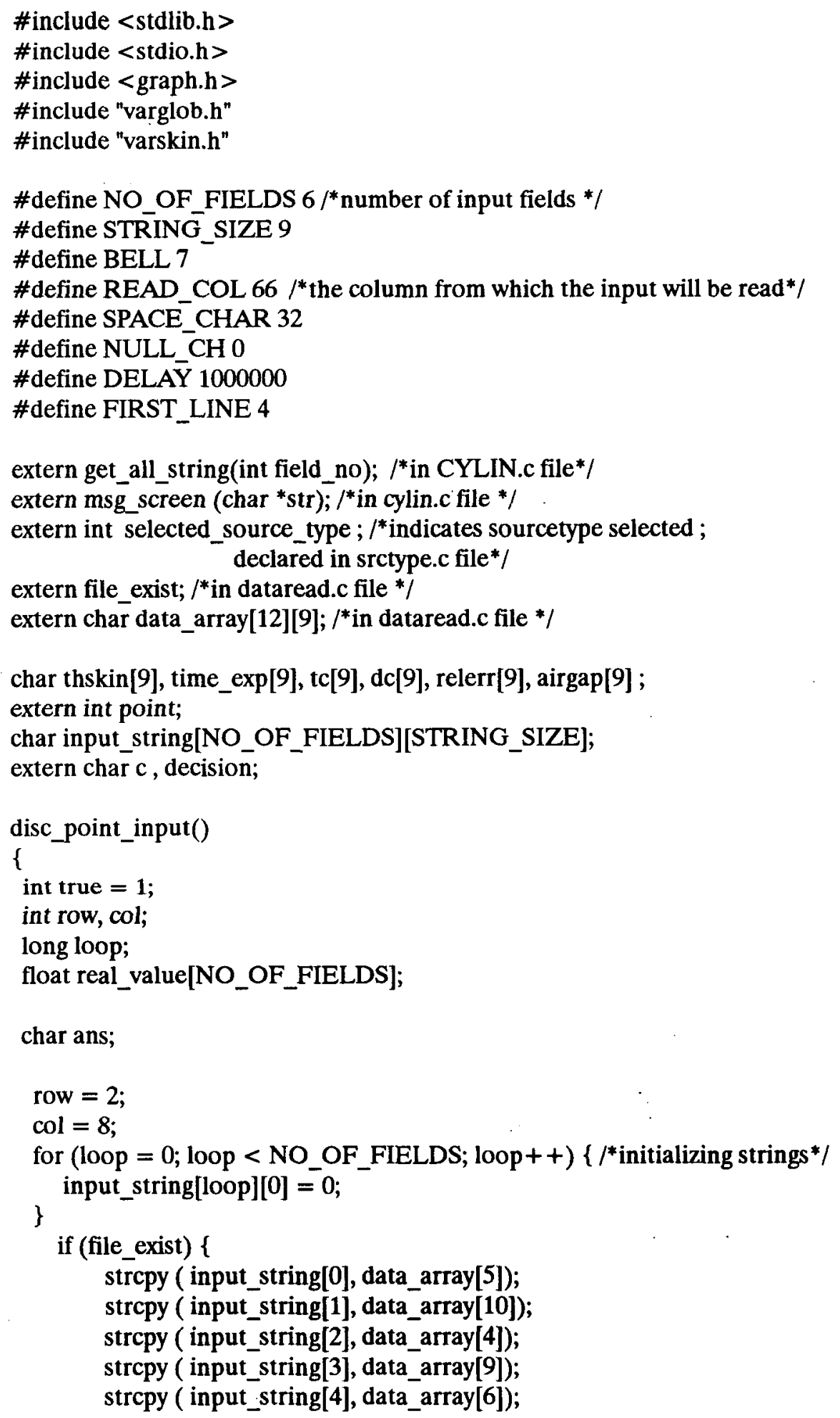


strcpy (input_string[5], data_array[7]);

\}

_clearscreen (_GCLEARSCREEN);

settextposition( $1,(80$ - strlen("POINT SOURCE GEOMETRY"))/2);

printf("POINT SOURCE GEOMETRY");

settextposition (row $+=2$, col);

printf ("Enter the skin density thickness in $\mathrm{mg} / \mathrm{cm}^{\wedge} 2 \quad$ : ");

Settextposition (row $+=2$, col);

printf ("Enter the exposure time in minutes : : ");

_settextposition (row $+=2$, col);

printf ("Enter the relative error

_settextposition (row $+=2$, col);

printf ("Enter the air gap thickness in $\mathrm{mm} \quad$ : ");

settextposition (row $+=2$, col);

printf ("Enter the thickness of material between the source and : ");

_settextposition (row $+=1$, col);

printf (" skin (protective clothing) in $\mathrm{mm}^{\prime \prime}$ );

settextposition (row $+=1$, col);

printf ("Enter the density of covering material in $\mathrm{g} / \mathrm{cm}^{\wedge} 3 \quad$ : ");

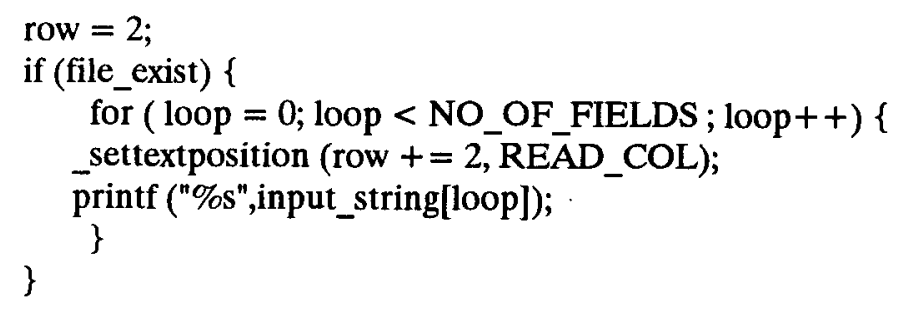

while (true) \{

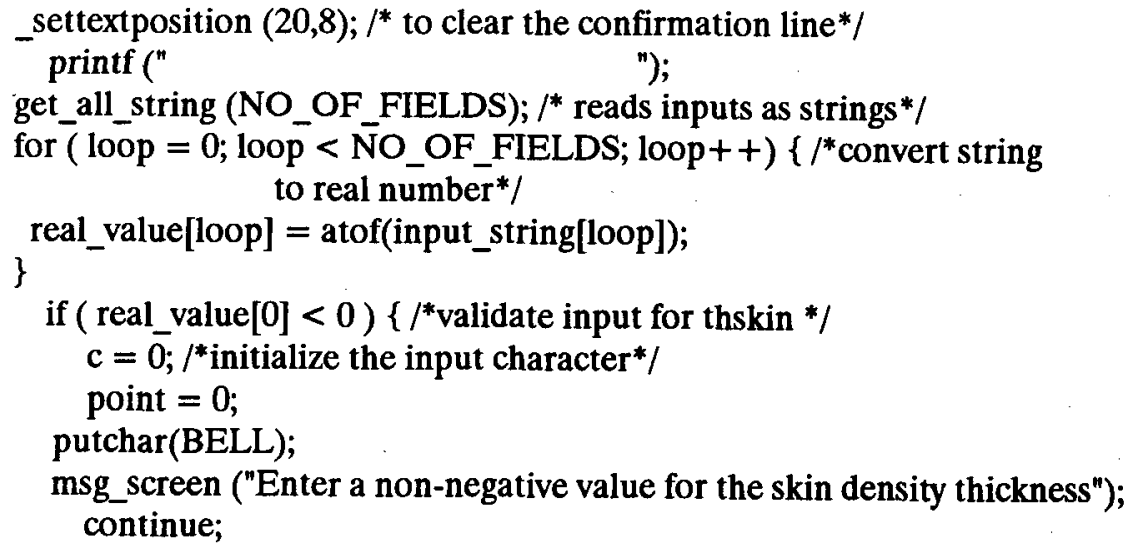




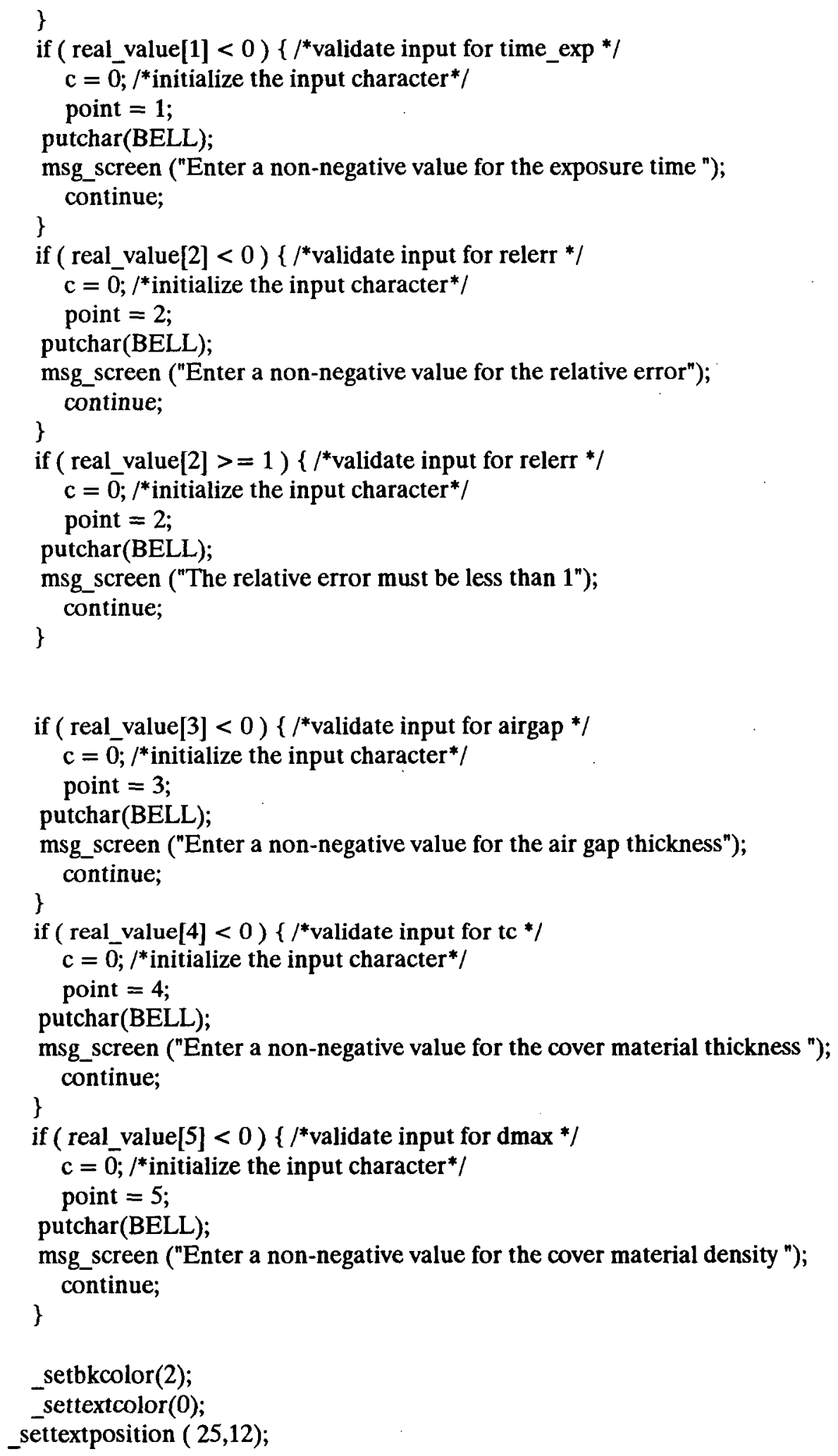




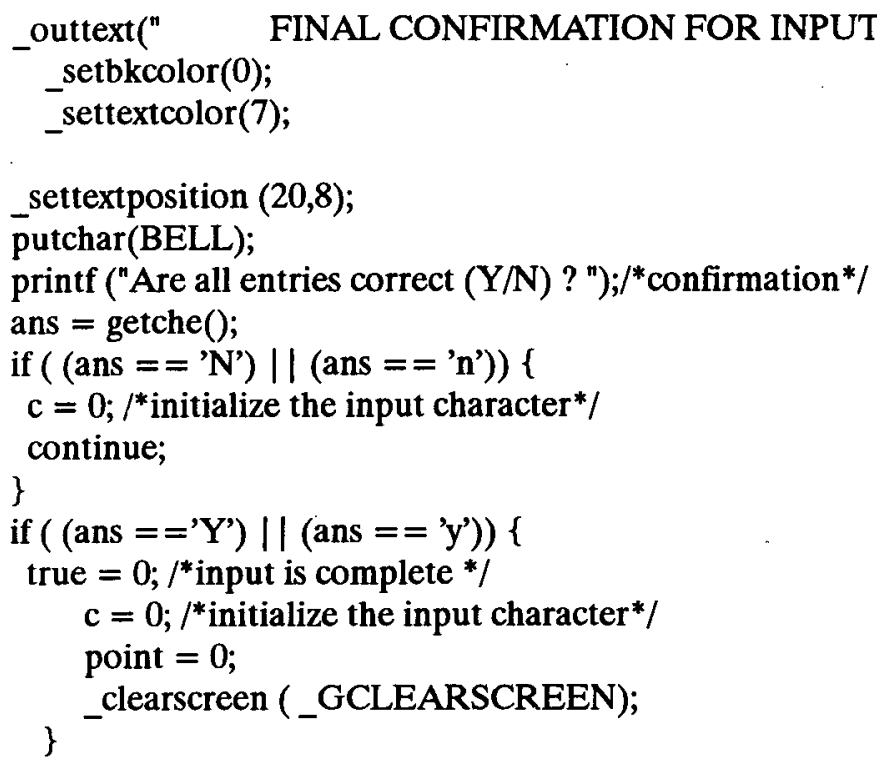


PROGRAM MODULE DISC.C

\#include <stdlib.h $>$ \#include <stdio.h $>$

\#include < graph.h> \#include "varglob.h"

\#include "varskin.h"

\#define NO_OF_FIELDS $8 /{ }^{*}$ number of input fields */

\#define STRING_SIZE 9

\#define BELL 7

\#define READ_COL $66 / *$ the column from which the input will be read*/

\#define SPACE_CHAR 32

\#define NULL_CH 0

\#define DELAY 100000

\#define FIRST_LINE 4

\#define PI $3.14 \overline{2} 8$

extern get_all_string(int field_no); $/ *$ in CYLIN.c file* $/$

extern msg_screen (char *str); $/ *$ in cylin.c file */

extern int selected_source_type ; $/ *$ indicates sourcetype selected ; declared in srctype.c file*/

extern file exist; $/{ }^{*}$ in dataread.c file */

extern char data_array[12][9]; /*in dataread.c file */

char diameter[9], thskin[9], time_exp[9], tc[9], dc[9], relerr[9], airgap[9],

area_exp[9] ;

extern int point;

char input_string[NO_OF_FIELDS][STRING_SIZE];

extern char c , decision;

disc_input()

\{

int true $=1$;

int row, col;

long loop;

float real_value[NO_OF_FIELDS];

float area_value;

char ans;

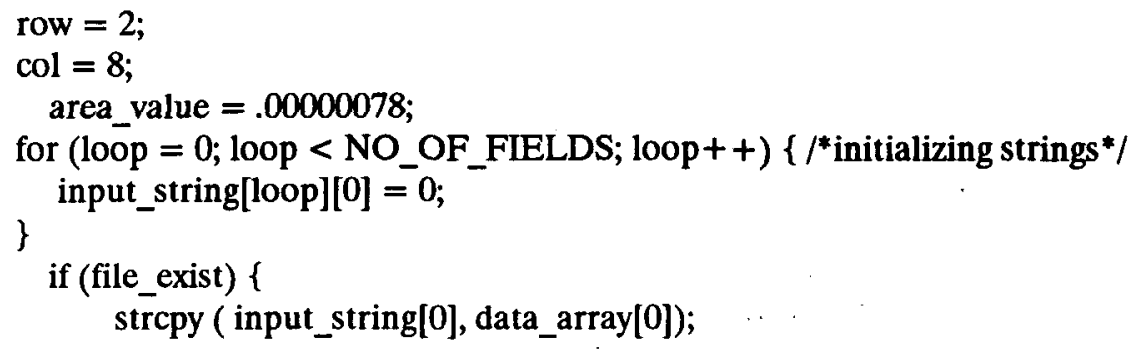


strcpy (input_string[1], data_array[11]); strcpy (input_string[2], data_array[5]); strcpy (input_string[3], data_array[10]); strcpy (input_string[4], data_array[4]); strcpy (input_string[5], data_array[9]); strcpy (input_string[6], data_array[6]); strcpy (input_string[7], data_array[7]);

\}

_clearscreen (_GCLEARSCREEN); settextposition( 1, (80 - strlen("2-D DISC GEOMETRY"))/2); printf("2-D DISC GEOMETRY"); settextposition (row $+=2$, col);

printf ("Enter the 2-D disk diameter in micrometers : "); settextposition (row $+=2$, col);

printf ("Enter the 2-D disk area in $\mathrm{cm}^{\wedge} 2$ : "); settextposition (row $+=2$, col);

printf ("Enter the skin density thickness in $\mathrm{mg} / \mathrm{cm}^{\wedge} 2 \quad:$ :); settextposition (row $+=2$, col);

printf ("Enter the exposure time in minutes : "); settextposition (row $+=2$, col); printf ("Enter the relative error _settextposition (row $+=2, \mathrm{col}$ ); printf ("Enter the air gap thickness in $\mathrm{mm} \quad$ :"); _settextposition (row $+=2$, col);

: ");

printf ("Enter the thickness of material between the source and : "); settextposition (row $+=1$, col);

printf (" skin (protective clothing) in $\mathrm{mm}^{\prime \prime}$ ); settextposition (row $+=1, \mathrm{col}$ );

printf ("Enter the density of covering material in $\mathrm{g} / \mathrm{cm}^{\wedge} 3 \quad:$ ");

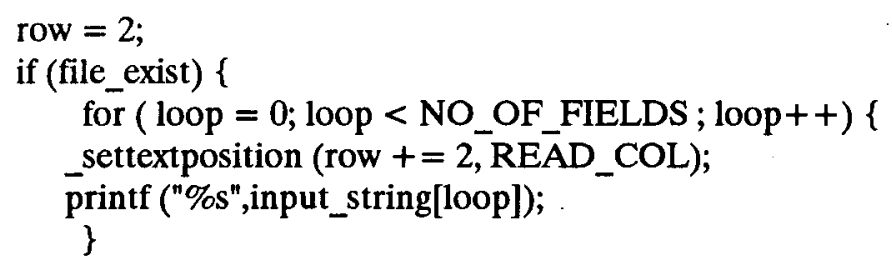

while (true) \{

_settextposition $(20,8) ; /^{*}$ to clear the confirmation line $* /$

printf (" ");

get_all_string (NO_OF_FIELDS); /* reads inputs as strings*/

for $($ loop $=0$; loop $<$ NO_OF_FIELDS; loop ++$)\{/ *$ convert string 


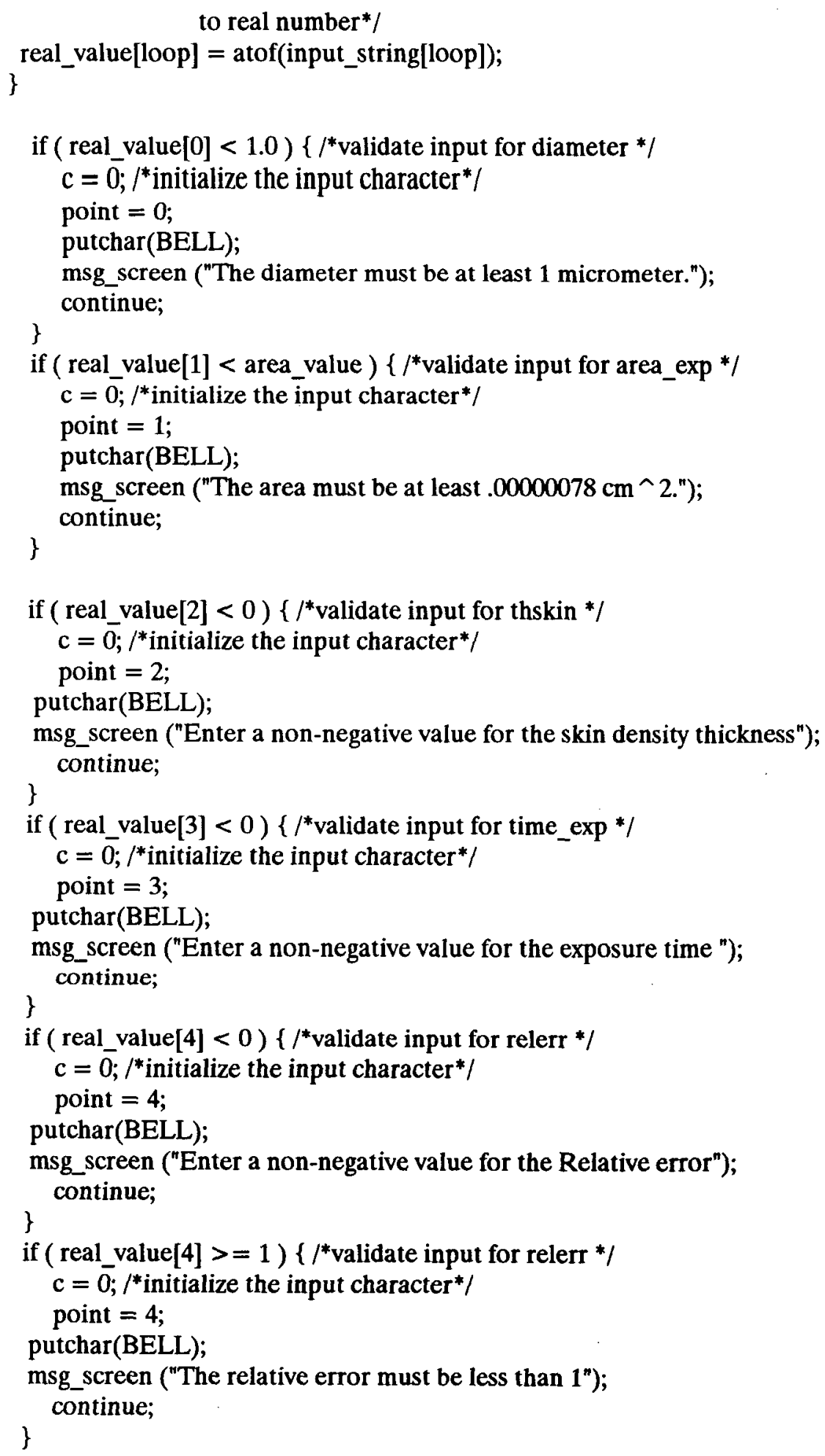




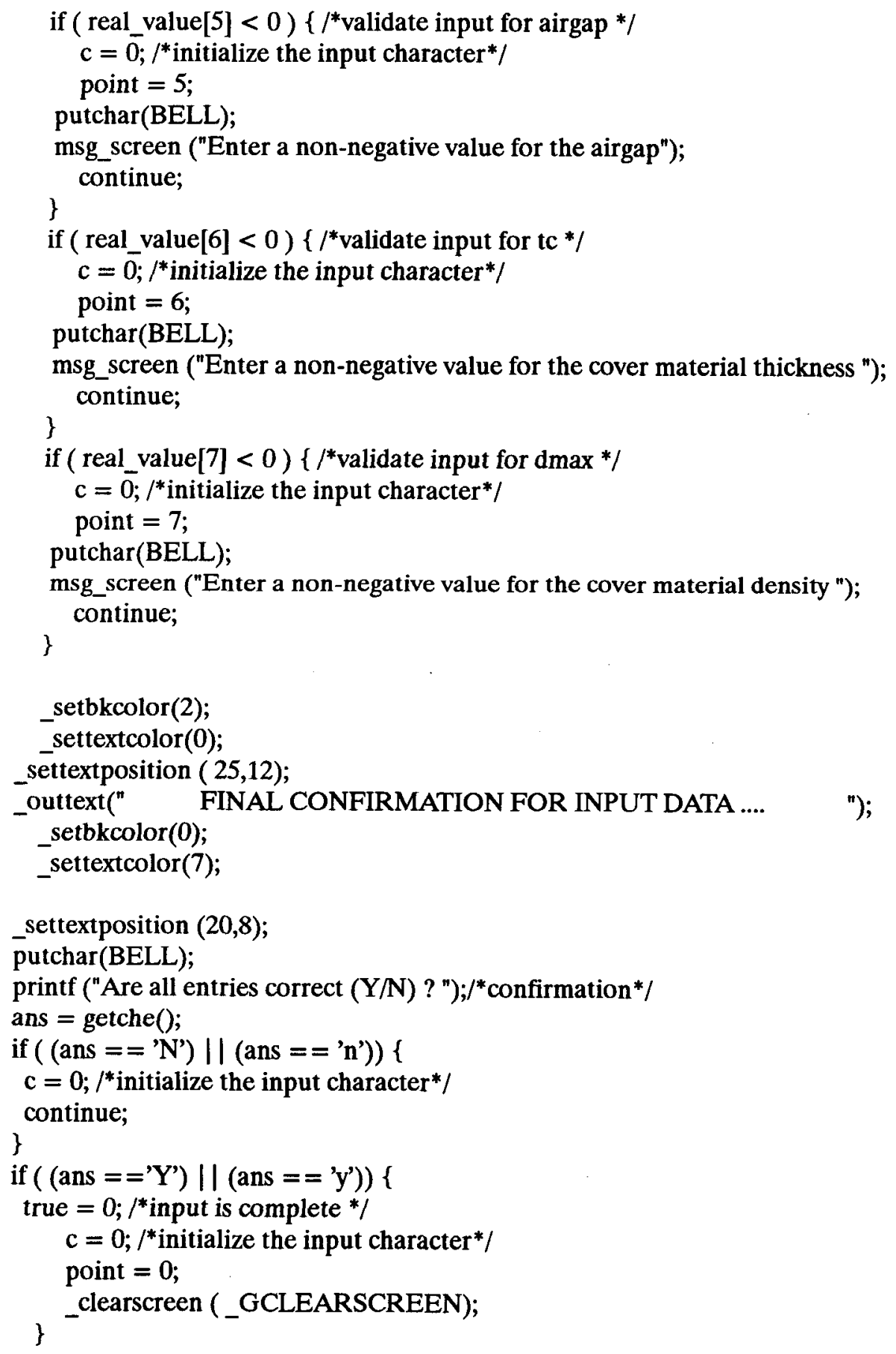


strcpy (data_array[4], input_string[4]); strcpy (data_array[9], input_string[5]); strcpy (data_array[6], input_string[6]); strcpy (data_array[7], input_string[7]);

strcpy (diameter, input_string[0]); strcpy (area_exp, input_string[1]); strcpy (thskin, input_string[2]);

strcpy ( time_exp, input_string[3]);

strcpy ( relerr, input_string[4]);

strcpy (airgap, input_string[5]);

strcpy ( tc , input_string[6]);

strcpy ( dc , input_string[7]); 
Appendix D

\section{PROGRAM MODULE CYLIN.C}

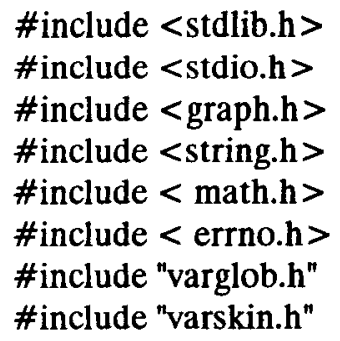

\#define NO_OF_FIELDS $9 / *$ number of input fields */

\#define STRING_SIZE 9

\#define BELL 7

\#define READ_COL $66 / *$ the column from which the input will be read*/

\#define SPACE_CHAR 32

\#define NULL_CH 0

\#define DELAȲ 1000000

\#define FIRST_LINE 4

\#define PI 3.1415926

extern VARSKIN_DATA_TYPE varskin_data; $\quad l^{*}$ definition in varskin.h file */

extern float xninty[6];

extern window( int window_row1, int window_col1, int window_row2, int window_col2, char attr);

$/ *$ in testscr4.c file */

extern help_screen (char *string); $/ *$ in help.c file */

extern file_exist; /*in dataread.c file */

extern char data_array[12][9]; /*in dataread.c file */

extern int selected_source_type; /*indicates sourcetype selected;

decalred in src.c file*/

char diameter[9], dth1 [9], time_exp[9], dens[9], relerr[9], dmax1[9],

airgap [9],tc[9], dc[9] ;

int point $=0$;

char input_string[NO_OF_FIELDS][STRING_SIZE];

char $\mathrm{c}=0$, decision;

cylin_input()

\{

int true = 1 ;

int row, col, $f$, invalid_dmax, iteration;

long loop;

float real_value[NO_OF_FIELDS];

char ans;

$$
\text { row }=2 \text {; }
$$

$\operatorname{col}=8$; 

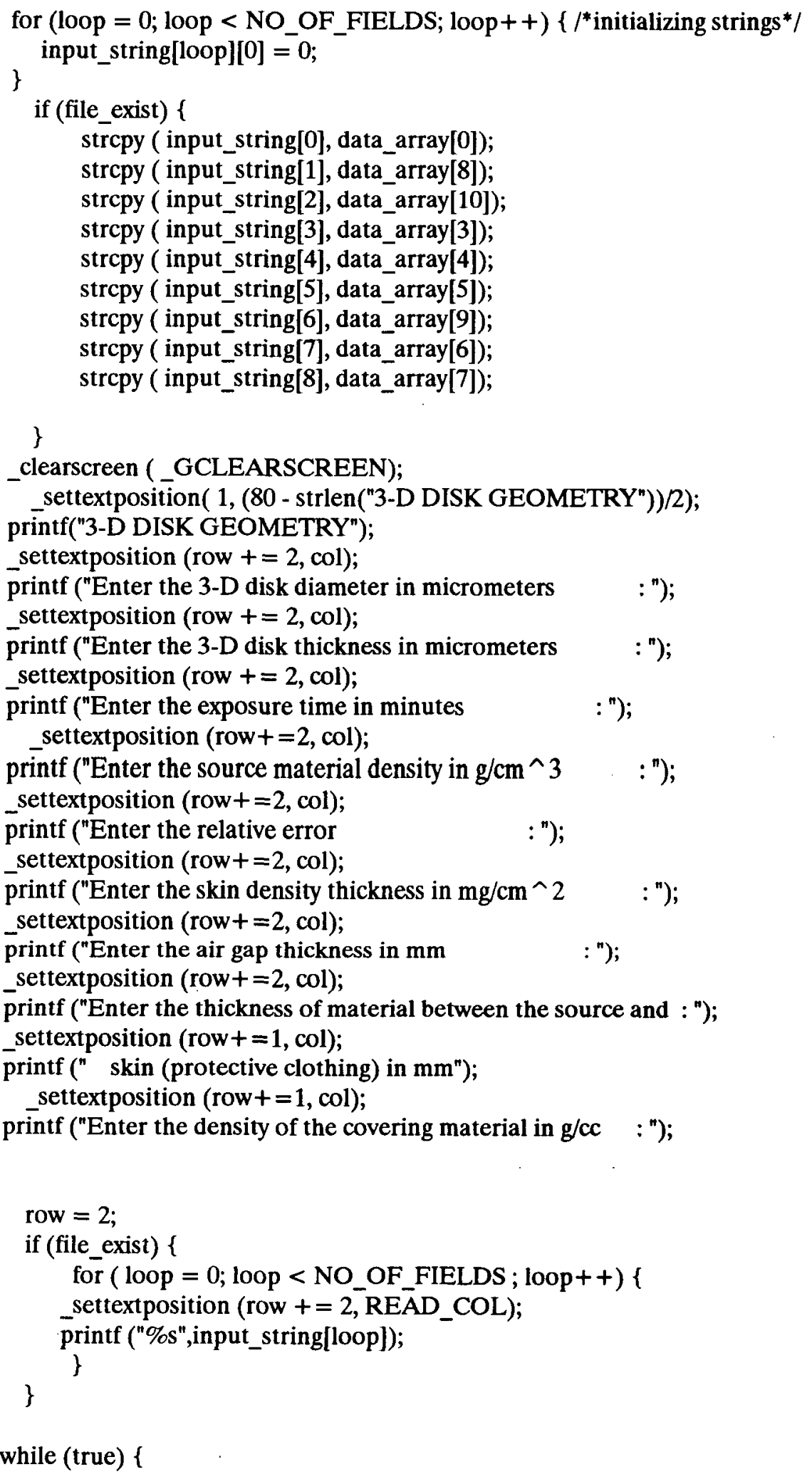
Appendix D

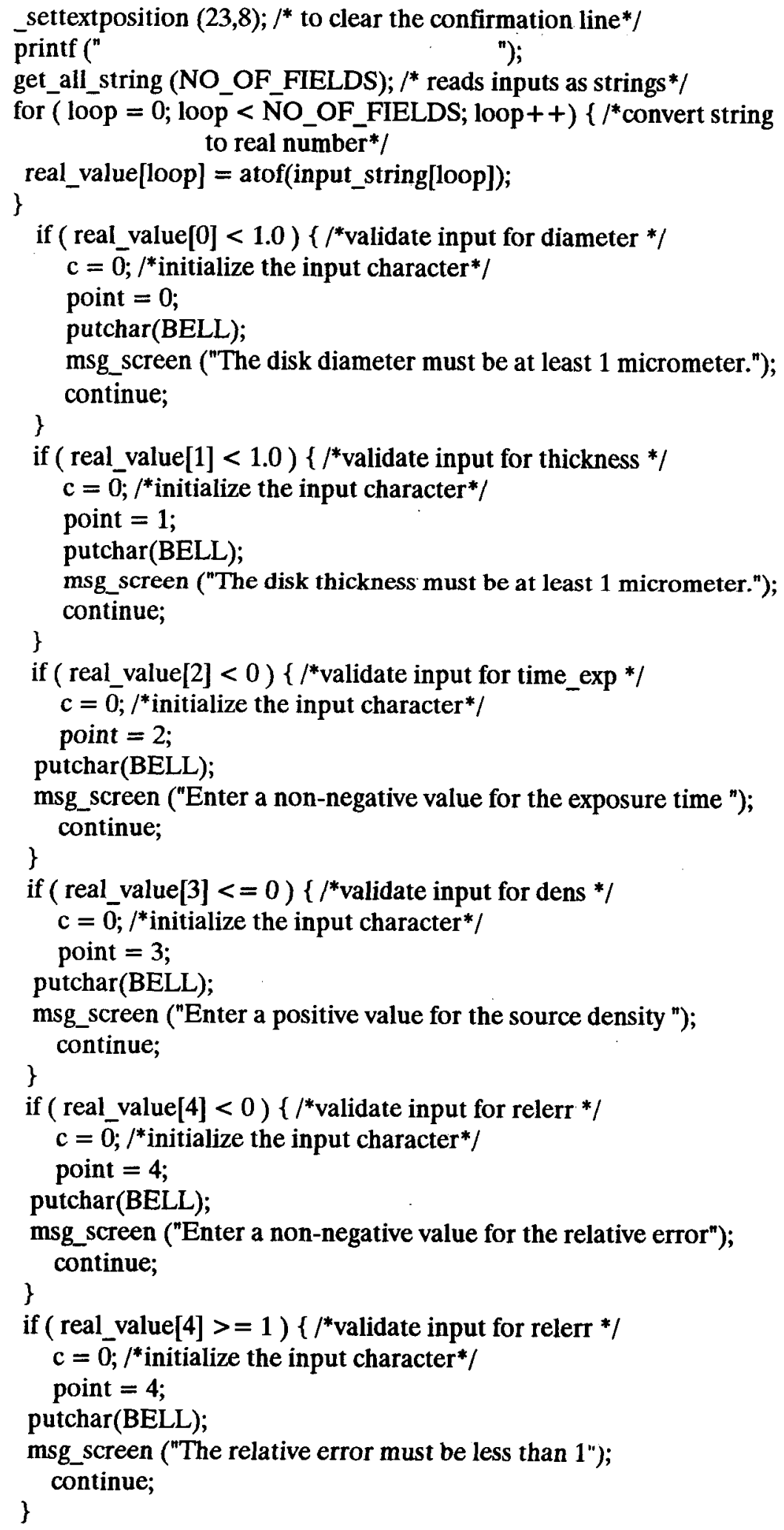




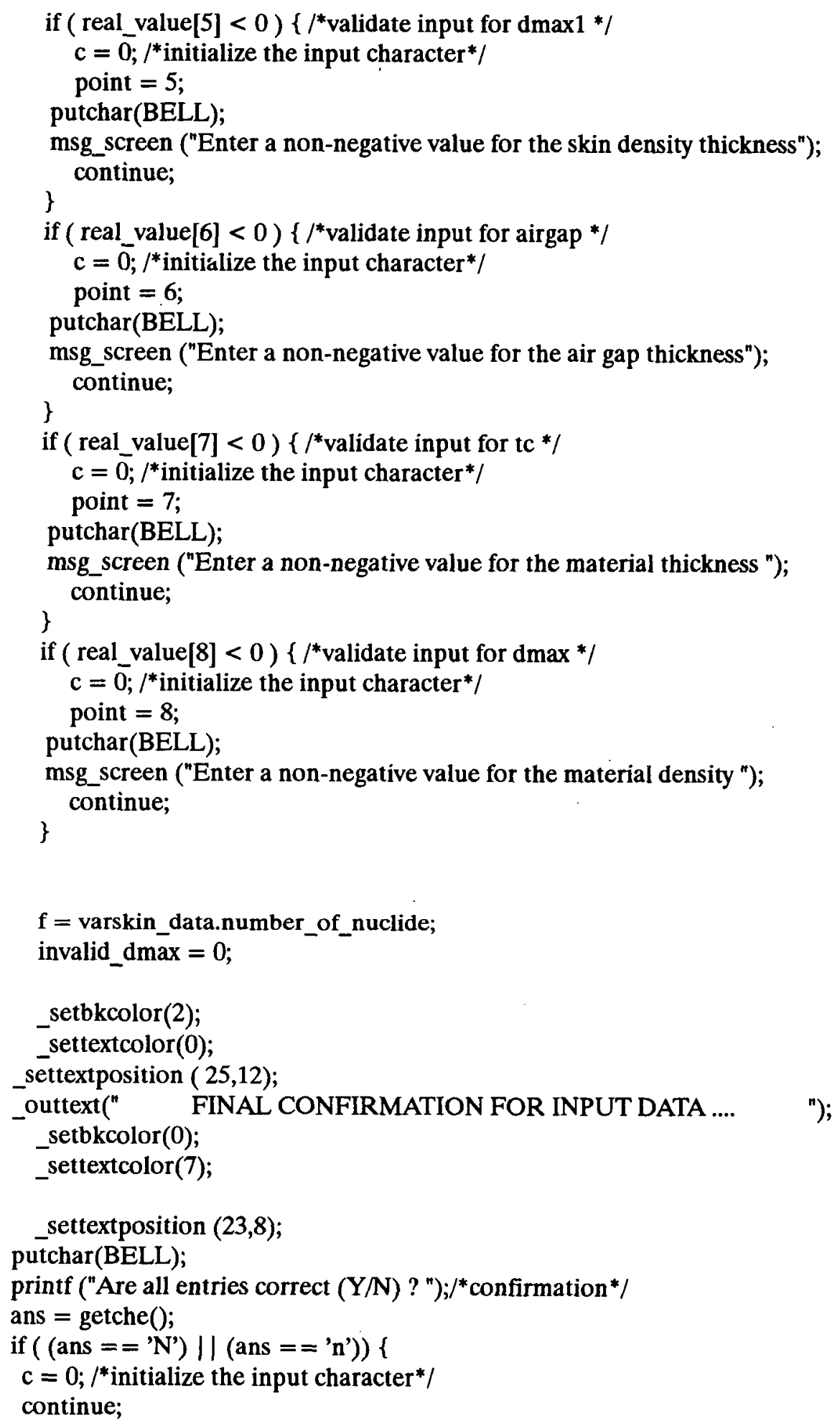


Appendix D

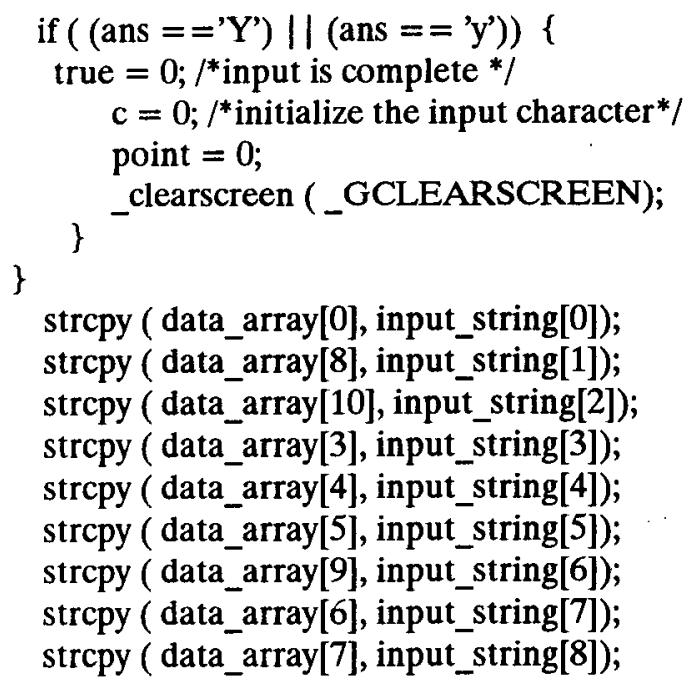

strcpy ( diameter, input_string[0]);

strcpy (dth1, input_string[1]);

strcpy ( time_exp, input_string[2]);

strcpy ( dens, input_string[3]);

strcpy ( relerr , input_string[4]);

strcpy (dmax1 , input_string[5]);

strcpy ( airgap, input_string[6]);

strcpy ( tc , input_string[7]);

strcpy ( dc , input_string[8]);

\}

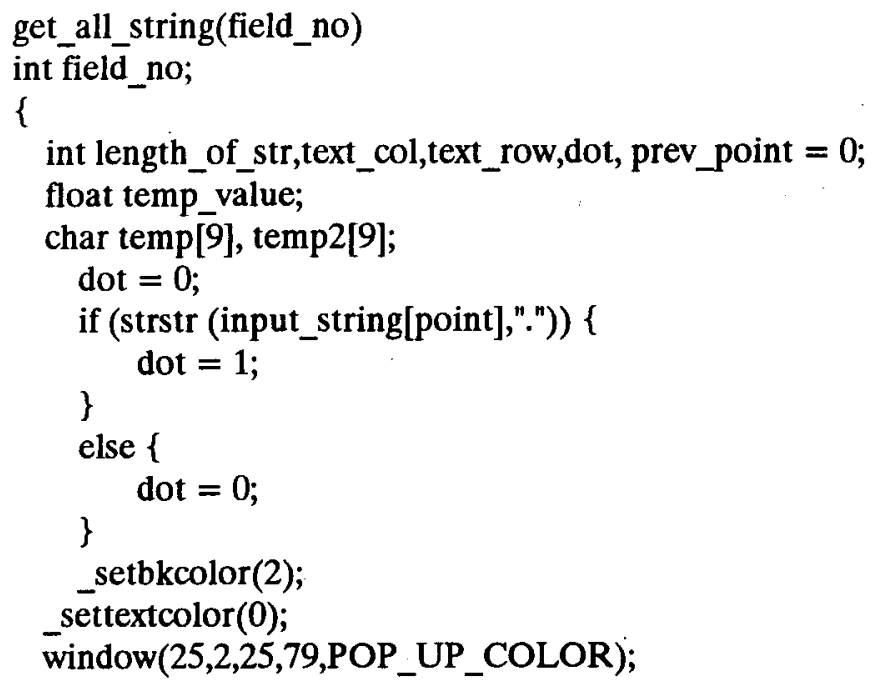




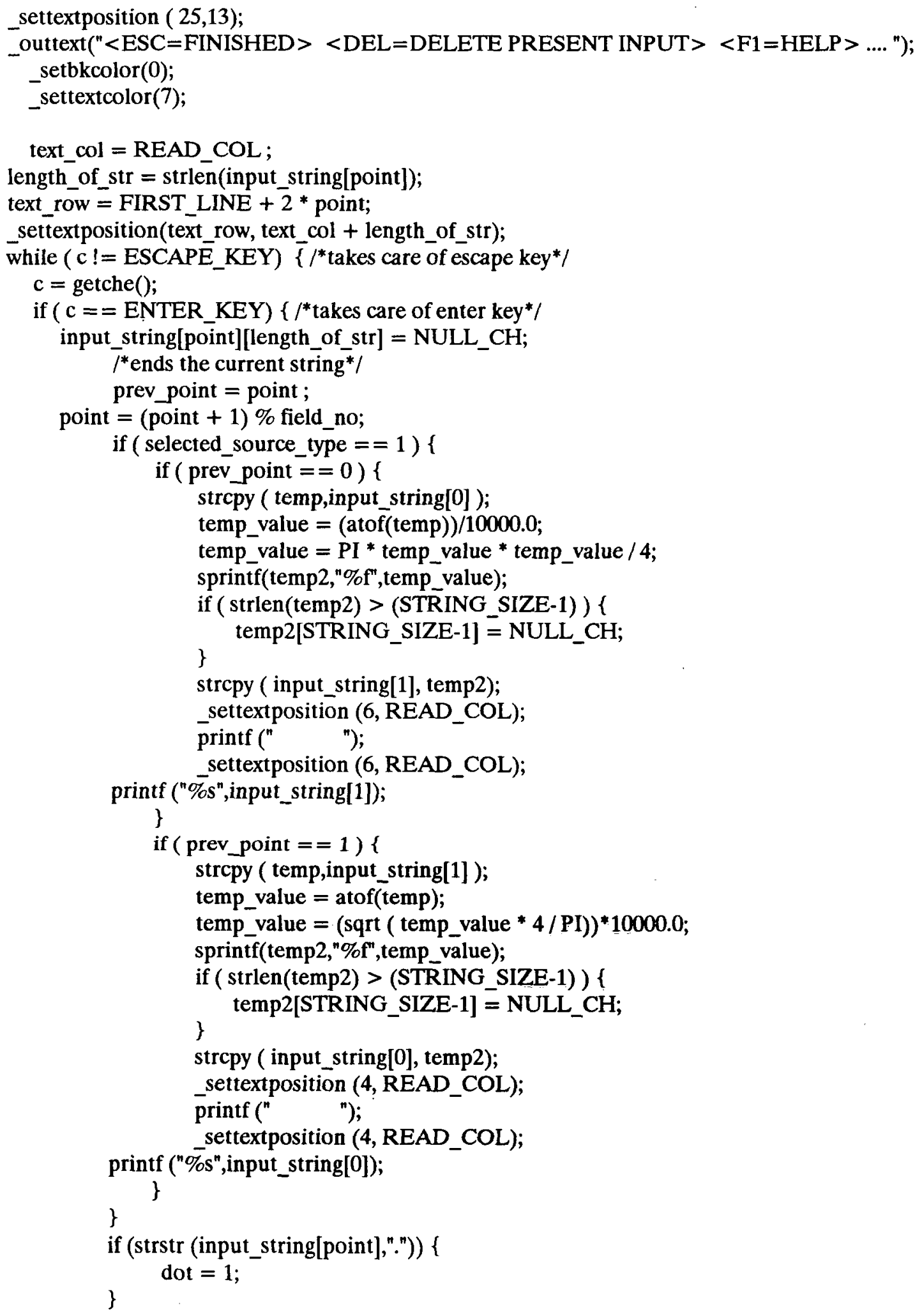


Appendix D

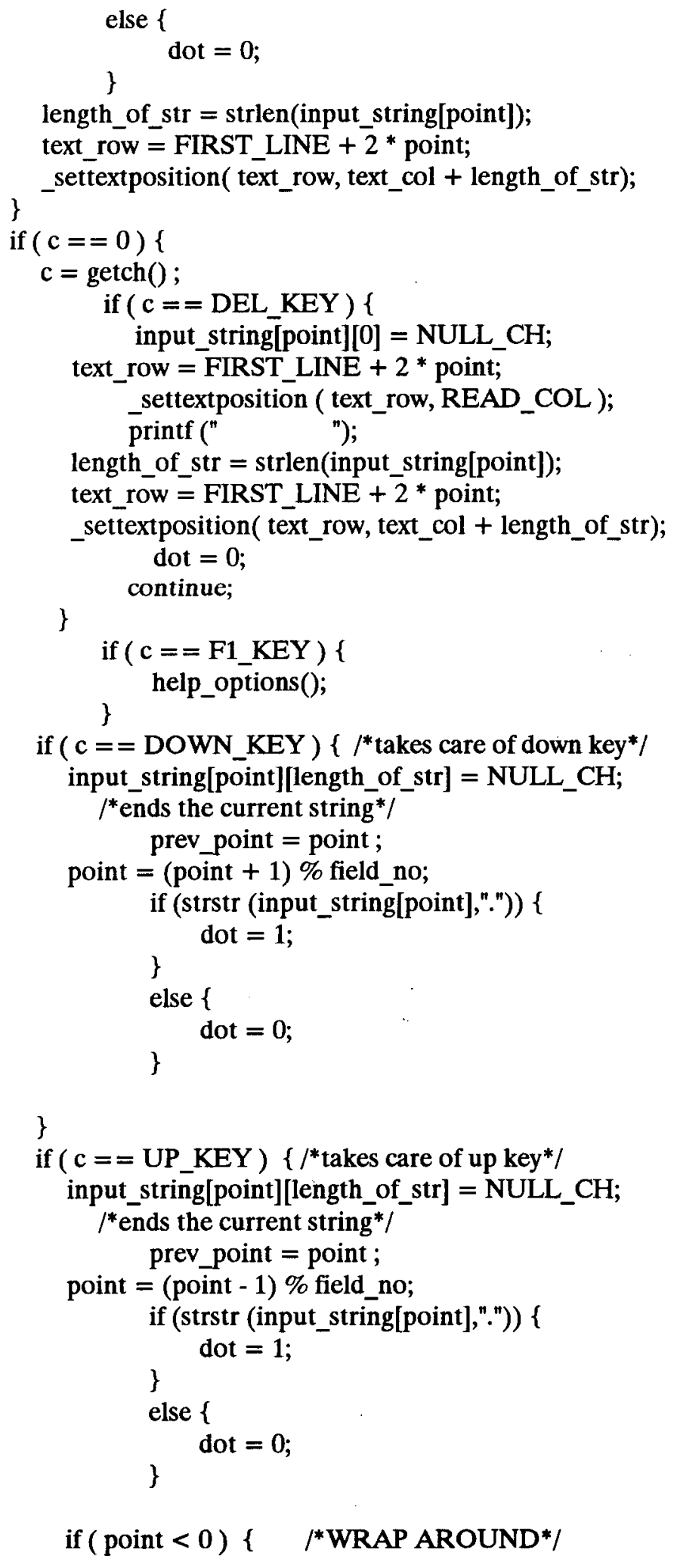




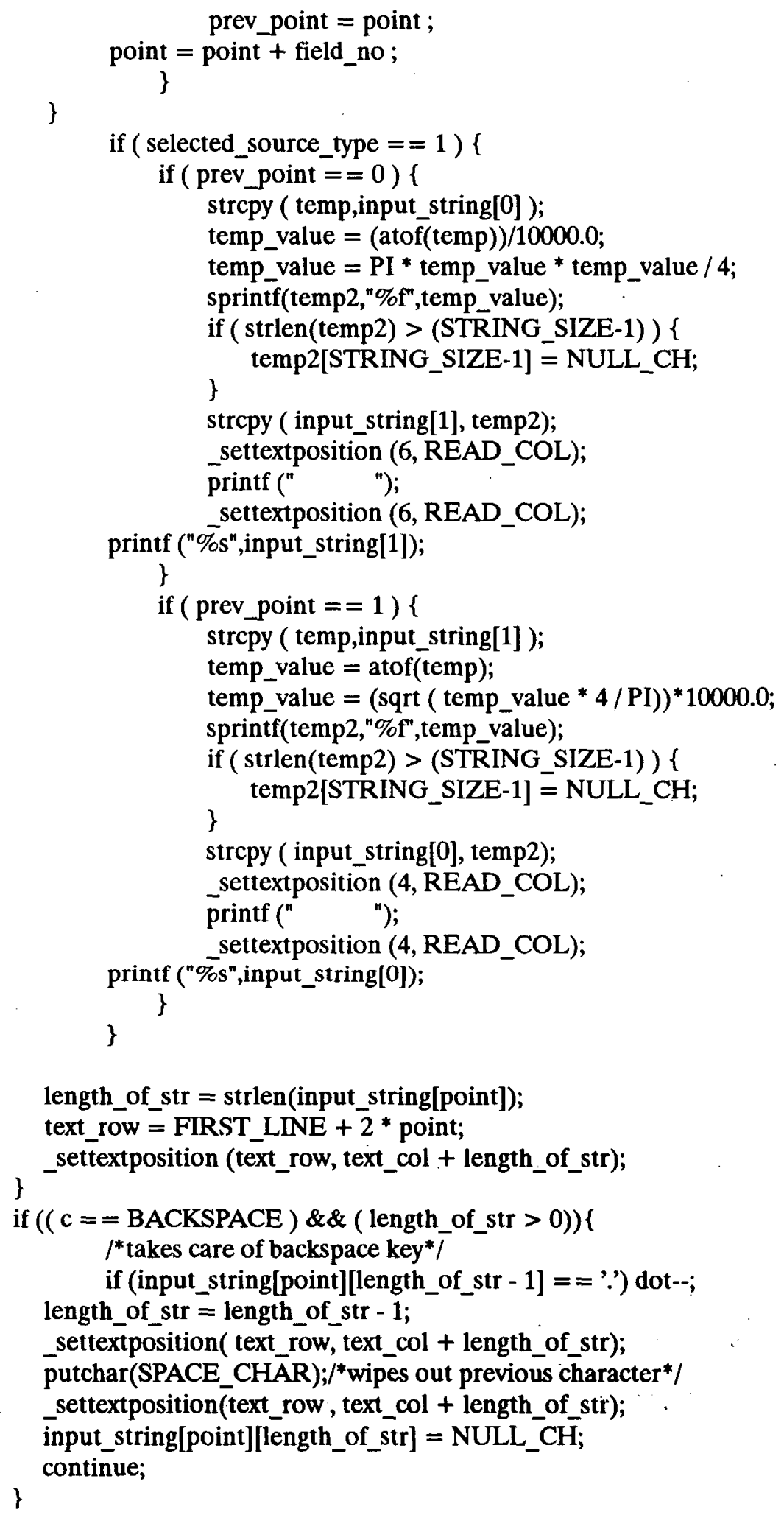


Appendix D

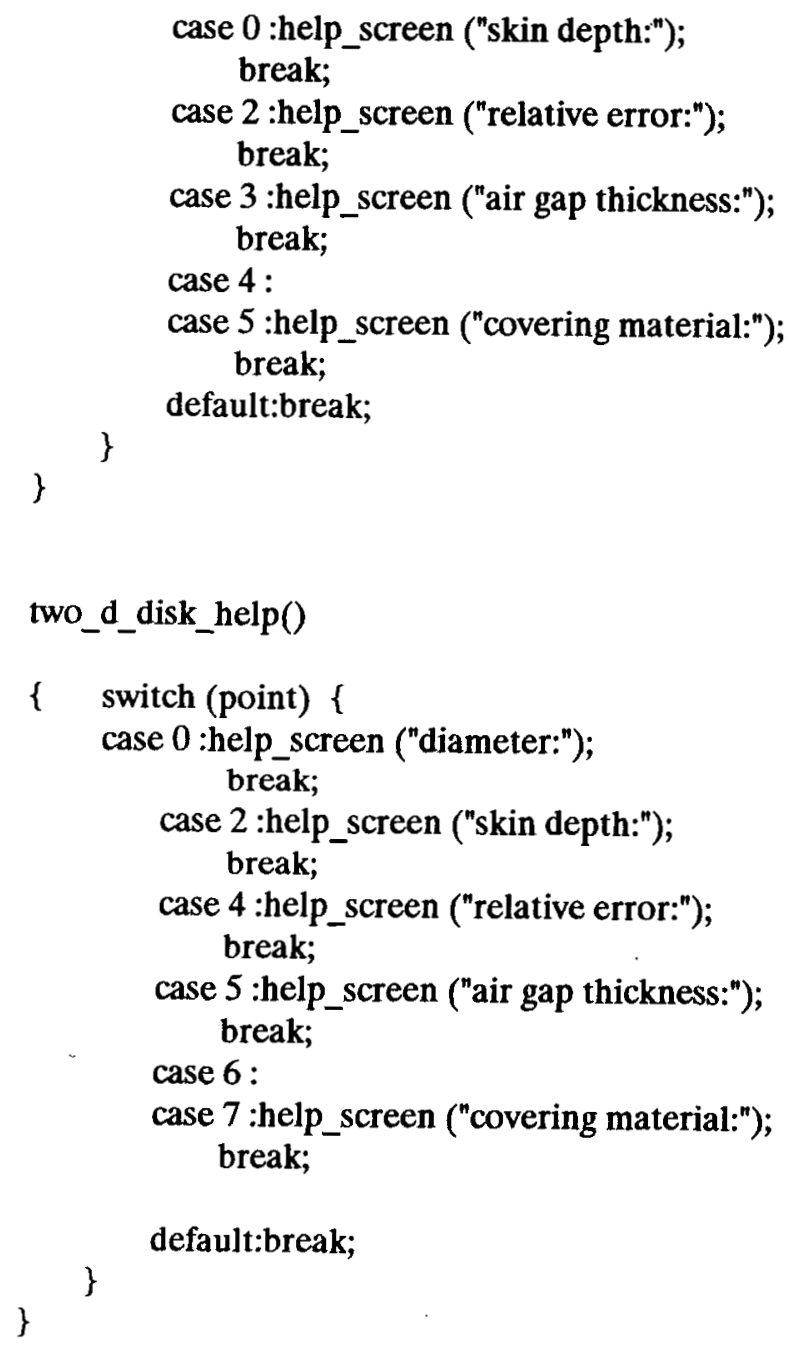




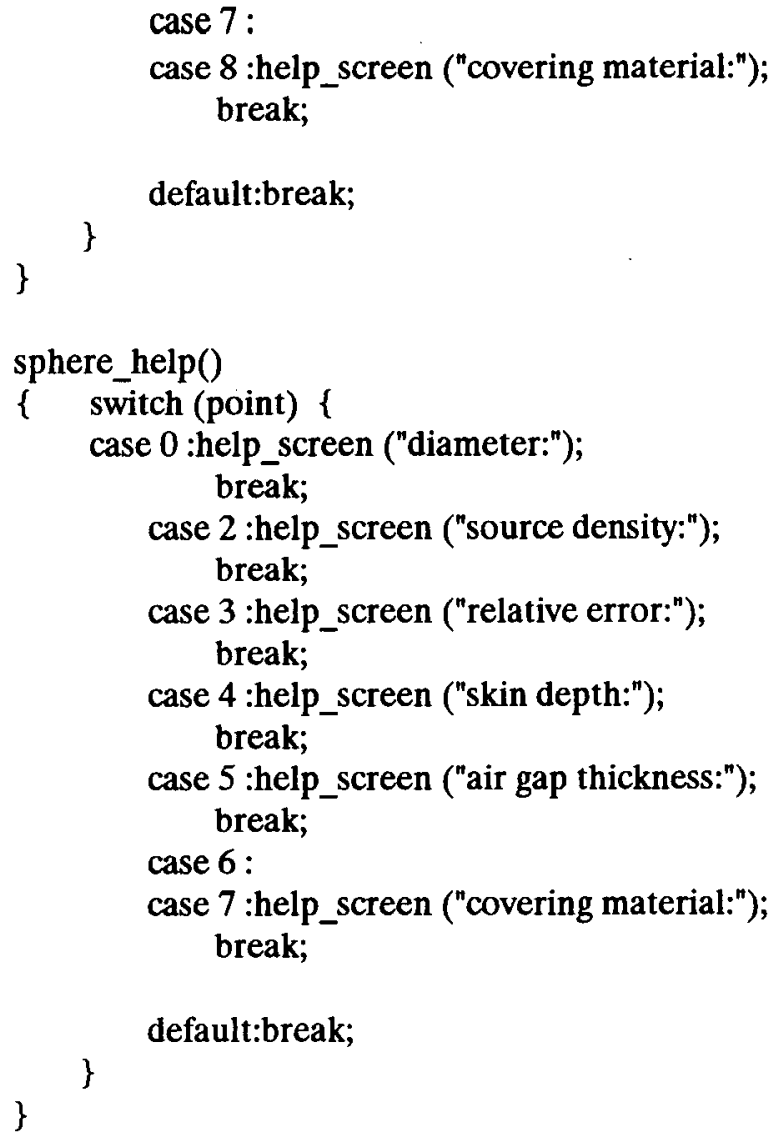




\section{Appendix D}

\}

\}

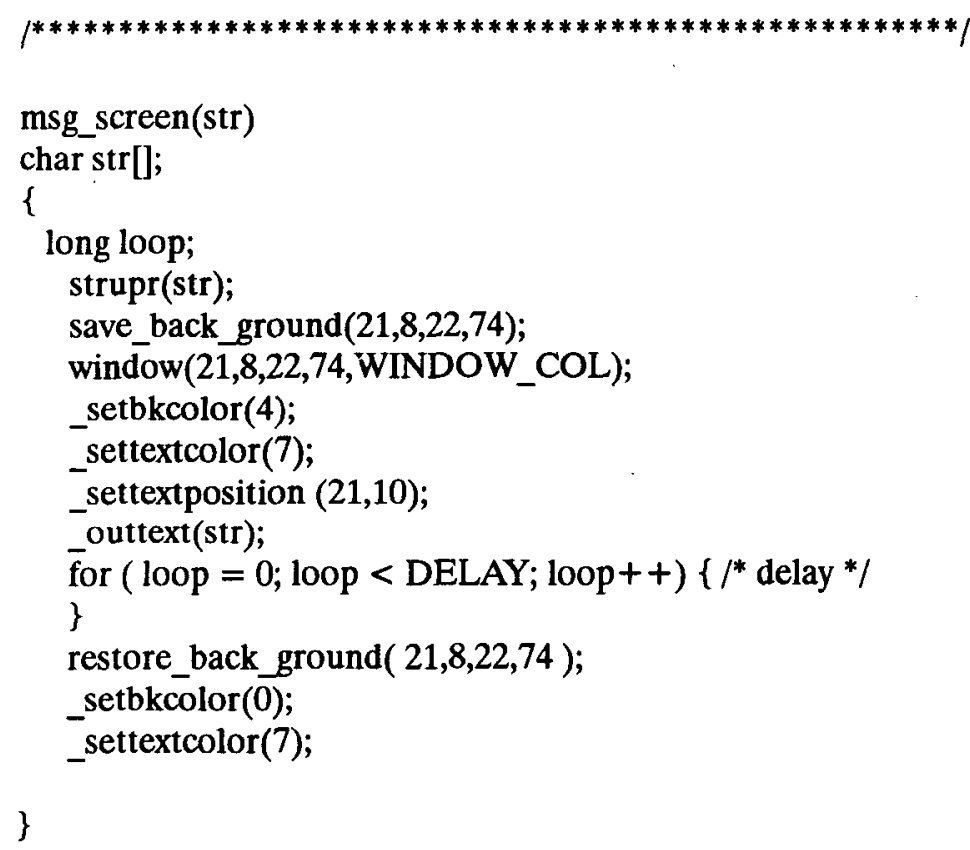




\section{PROGRAM MODULE SPHERE.C}

\#include $<$ stdlib.h $>$

\#include <stdio.h $>$

\#include < graph.h >

\#include "varglob.h"

\#include "varskin.h"

\#define NO_OF_FIELDS $8 /$ *number of input fields */

\#define STRING_SIZE 9

\#define BELL 7

\#define READ_COL $66 / /^{*}$ the column from which the input will be read*/

\#define SPACE_CHAR 32

\#define FIRST_LINE $4 / *$ first line of input */

\#define NULL_CH 0

\#define DELAY 1000000

extern VARSKIN_DATA_TYPE varskin_data; $\quad I^{*}$ definition in varskin.h file * $/$

extern get_all_string(int field_no);/*in C $\bar{Y} L I N . c$ file*/

extern msg_screen (char *str); $/{ }^{*}$ in CYLIN.c file*/

extern float xninty[6];

extern file exist; $/{ }^{*}$ in dataread.c file */

extern char data_array[12][9]; /*in dataread.c file */

char diameter[9], time_exp[9], dens[9], relerr[9], dmax1[9],

airgap [9], tc[9], dc[9] ;

extern int point ;

char input_string[NO_OF_FIELDS][STRING_SIZE];

extern char $\mathrm{c}$;

sphere_input()

\{

int true $=1$

int row, col, field_no, $f$, invalid_dmax,iteration ;

long loop;

float real_value[NO_OF_FIELDS];

char ans;

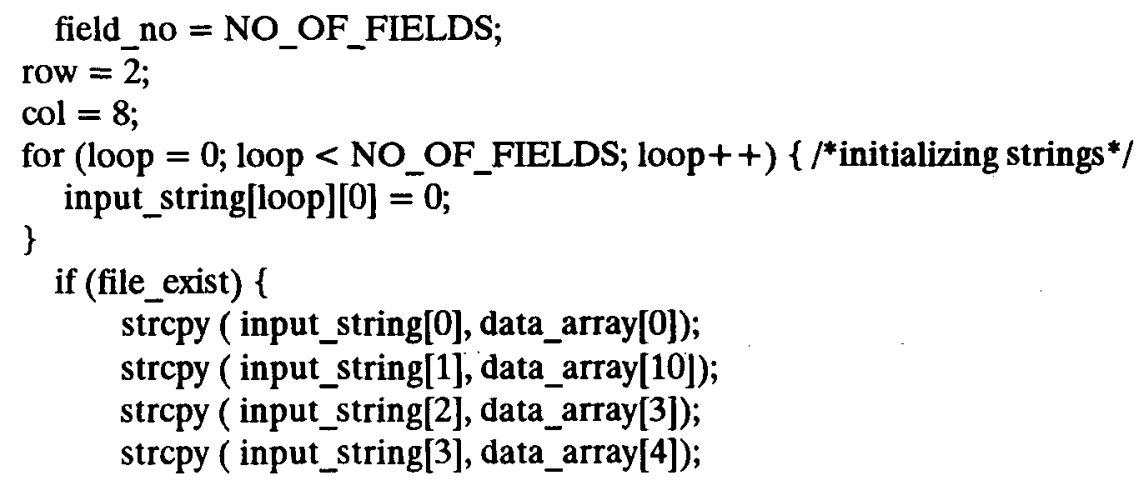


strcpy (input_string[4], data_array[5]);

strcpy (input_string[5], data_array[9]);

strcpy (input_string[6], data_array[6]);

strcpy (input_string[7], data_array[7]);

\}

_clearscreen (_GCLEARSCREEN);

settextposition( 1, (80 - strlen("SPHERICAL SOURCE GEOMETRY"))/2); printf("SPHERICAL SOURCE GEOMETRY");

settextposition (row $+=2$, col);

printf ("Enter the sphere diameter in micrometers : : ");

settextposition (row $+=2$, col);

printf ("Enter the exposure time in minutes : ");

settextposition (row $+=2, \mathrm{col}$ );

printf ("Enter the source material density in $\mathrm{g} / \mathrm{cm}^{-}$3 : ");

settextposition (row $+=2, \mathrm{col}$ );

printf ("Enter the relative error

settextposition (row $+=2$, col);

printf ("Enter the skin density thickness in $\mathrm{mg} / \mathrm{cm}^{\wedge} 2 \quad$ : ");

settextposition (row $+=2$, col);

printf ("Enter the air gap thickness in $\mathrm{mm} \quad$ : ");

_settextposition (row $+=2$, col);

printf ("Enter the thickness of material between the source and : ");

_settextposition (row $+=1$, col);

printf (" skin (protective clothing) in mm");

settextposition (row $+=1, \mathrm{col}$ );

printf ("Enter the density of the covering material in $\mathrm{g} / \mathrm{cm} \wedge 3$ : ");

row $=2$;

if (file_exist) \{

for $($ loop $=0$; loop < NO_OF_FIELDS ; loop ++$)\{$

settextposition (row $+=2, \overline{R E A}{ }_{-} \_C O L$ );

printf ("\%s",input_string[loop]);

\}

\}

while (true) \{

_settextposition $(22,8) ; /^{*}$ to clear the confirmation line*/

printf ("

");

get_all_string (field_no); ${ }^{*}$ reads inputs as strings*/

for $\left(100 p=0\right.$; loop $<$ NO_OF_FIELDS; loop ++ ) $\left\{/{ }^{*}\right.$ convert string

to real number*/

real_value[loop] = atof(input_string[loop]);

\}

if ( real_value $[0]<1.0$ ) $\{/$ validate input for diameter */ $c=\overline{0} ; /^{*}$ initialize the input character* 


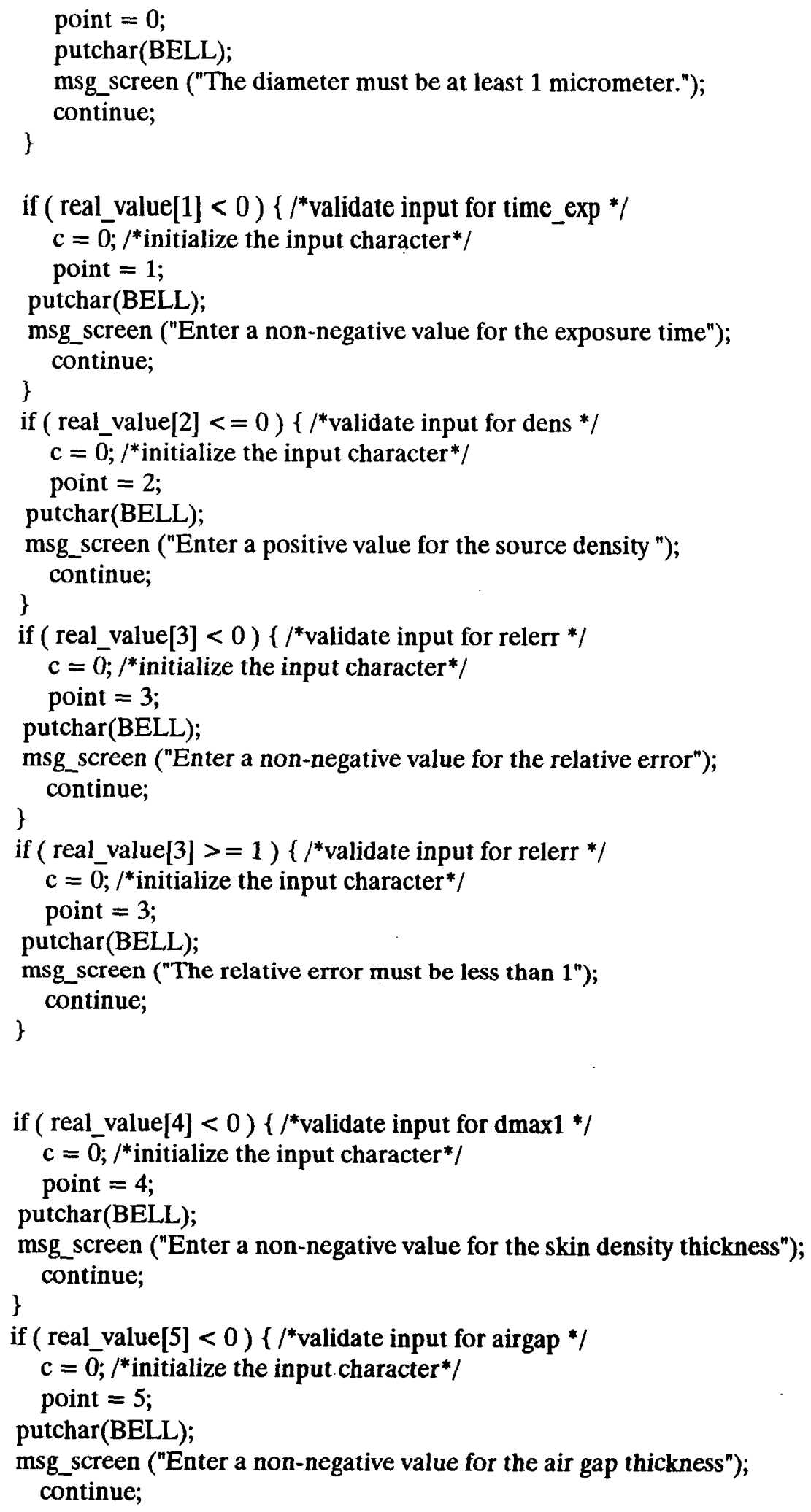




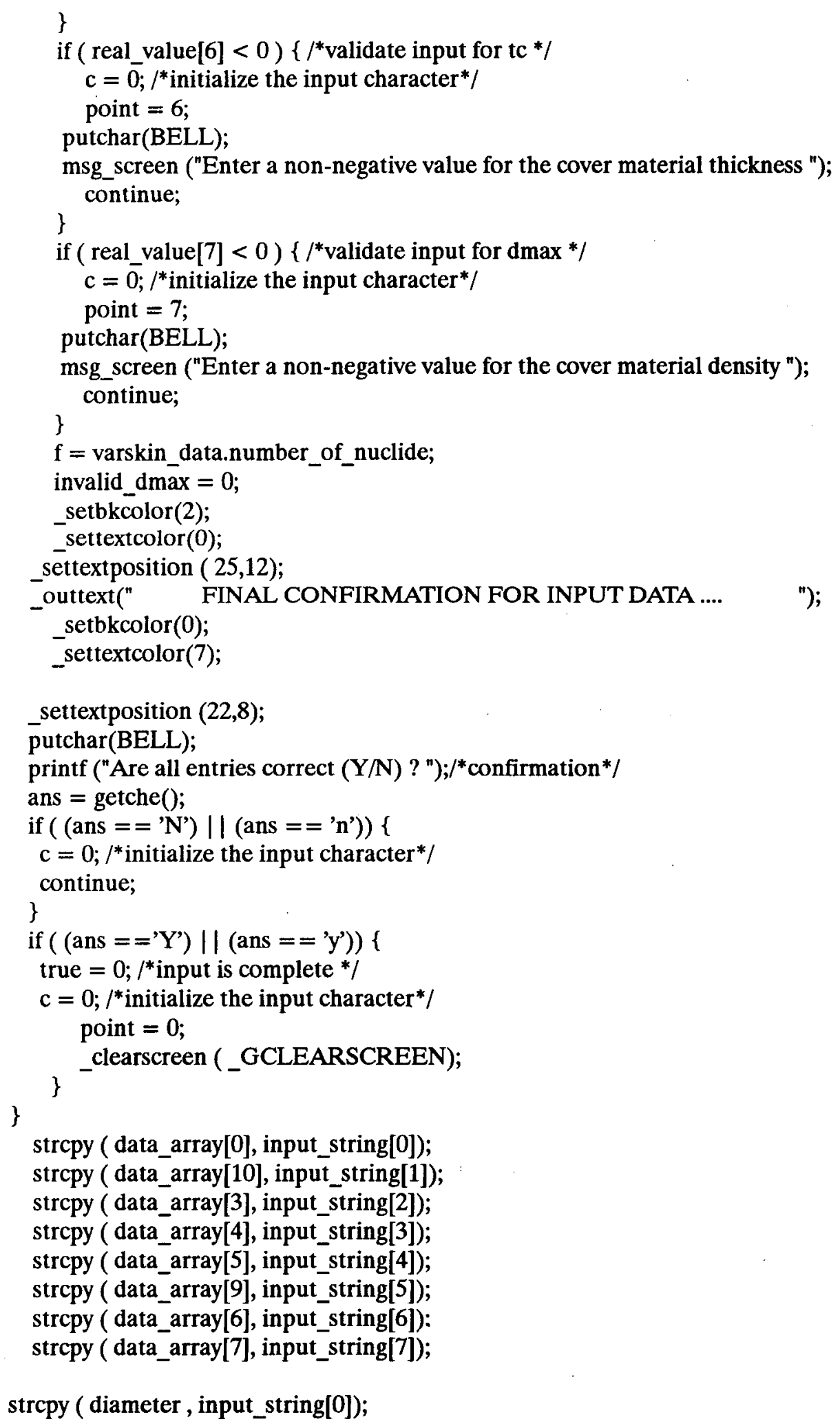


strcpy (time_exp, input_string[1]);

strcpy (dens , input_string[2]);

strcpy ( relerr, input_string[3]);

strcpy ( dmax 1, input_string[4]);

strcpy ( airgap, input_string[5]);

strcpy ( tc , input_string[6]);

strcpy (dc, input_string[7]); 
Appendix D

\section{PROGRAM SLAB.C}

\#include <stdlib.h>
\#include <stdio.h $>$
\#include < graph.h>
\#include "varglob.h"
\#include "varskin.h"

\#define NO_OF_FIELDS $10 /$ *number of input fields */

\#define STRING_SIZE 9

\#define BELL 7

\#define READ_COL $66 / *$ the column from which the input will be read*/

\#define SPACE_CHAR 32

\#define FIRST_LINE $4 / *$ first line of input */

\#define NULL $\mathrm{CH} 0$

\#define DELAȲ 1000000

extern VARSKIN_DATA_TYPE varskin_data; $\quad / *$ definition in varskin.h file */

extern get_all_string(int field_no);/*in CYYLIN.c file*/

extern ms $\bar{g}_{-}$screen (char *str); $/{ }^{*}$ in CYLIN.c file*/

extern float xninty[6];

extern file_exist; $/{ }^{*}$ in dataread.c file */

extern char data_array[12][9]; /*in dataread.c file */

char x_length[9],y_length[9], dth1[9], time_exp[9], dens[9], relerr[9],

dmax1 [9], airgap[9], tc[9], dc[9] ;

extern int point ;

char input_string[NO_OF_FIELDS][STRING_SIZE];

extern char c ;

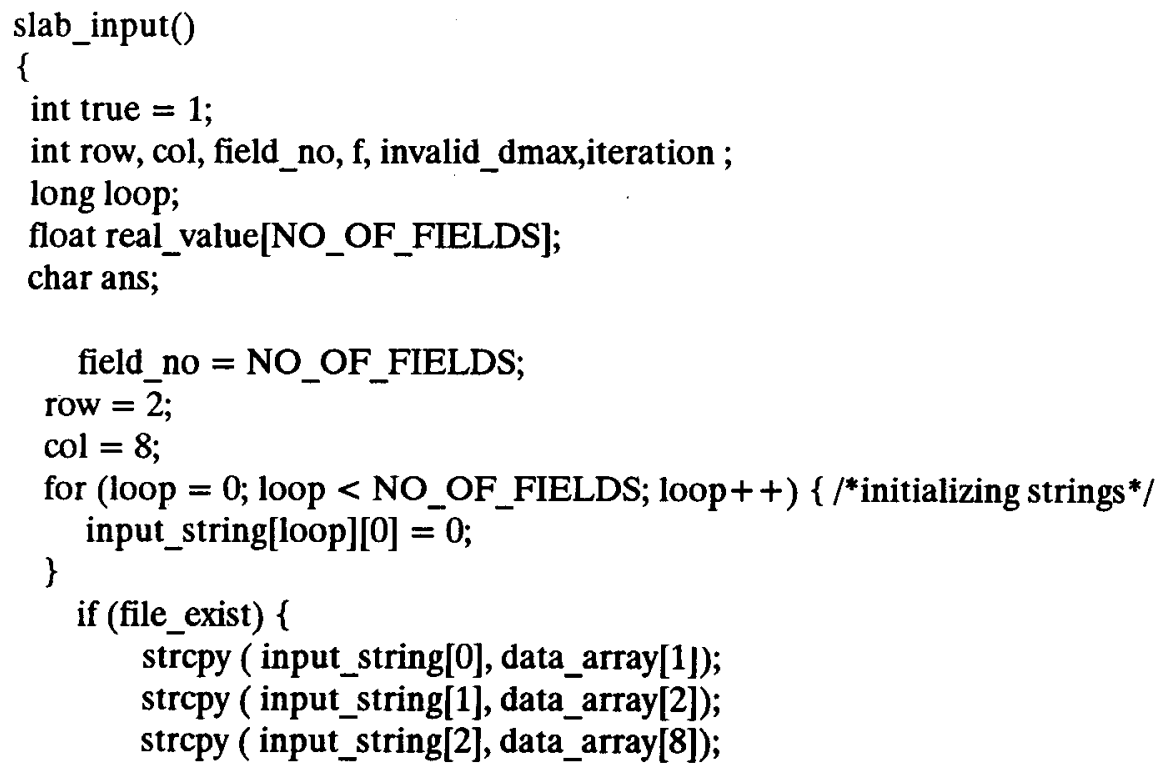


strcpy (input_string[3], data_array[10]);

strcpy (input_string[4], data_array[3]);

strcpy (input_string[5], data_array[4]);

strcpy (input_string[6], data_array[5]);

strcpy (input_string[7], data_array[9]);

strcpy (input_string[8], data_array[6]);

strcpy (input_string[9], data_array[7]);

\}

_clearscreen (_GCLEARSCREEN);

settextposition( 1, (80 - strlen("3-D SLAB GEOMETRY"))/2);

printf("3-D SLAB GEOMETRY");

settextposition (row $+=2$, col);

printf ("Enter the X-side length in micrometers : ");

settextposition (row $+=2$, col);

printf ("Enter the Y-side length in micrometers : ");

settextposition (row $+=2$, col);

printf ("Enter the slab thickness in micrometers : ");

settextposition (row $+=2$, col);

printf ("Enter the exposure time in minutes : ");

settextposition (row $+=2$, col);

printf ("Enter the source material density in $\mathrm{g} / \mathrm{cm}^{\wedge} 3 \quad:$ : );

settextposition (row $+=2$, col);

printf ("Enter the relative error : ");

settextposition (row $+=2, \mathrm{col}$ );

printf ("Enter the skin density thickness in $\mathrm{mg} / \mathrm{cm}^{\wedge} 2 \quad:$ ");

settextposition (row $+=2$, col);

printf ("Enter the air gap thickness in mm : ");

settextposition (row $+=2, \mathrm{col}$ );

printf ("Enter the thickness of material between the source and : ");

settextposition (row $+=1$, col);

printf (" skin (protective clothing) in $\mathrm{mm}^{\mathrm{n}}$ );

settextposition (row $+=1, \mathrm{col}$ );

printf ("Enter the density of covering material in $\mathrm{g} / \mathrm{cm}^{\wedge} 3 \quad:$ :);

row $=2$;

if (file_exist) \{

for $($ loop $=0$; loop < NO_OF_FIELDS ; loop ++ ) (

_settextposition (row $+=2$, READ_COL);

printf ("\%s",input_string[loop]);

\}

\}

while (true) \{

settextposition $(24,8) ; /^{*}$ to clear the confirmation line* ${ }^{*}$

printf ("

");

get_all_string (field_no); ${ }^{*}$ reads inputs as strings $* /$ 


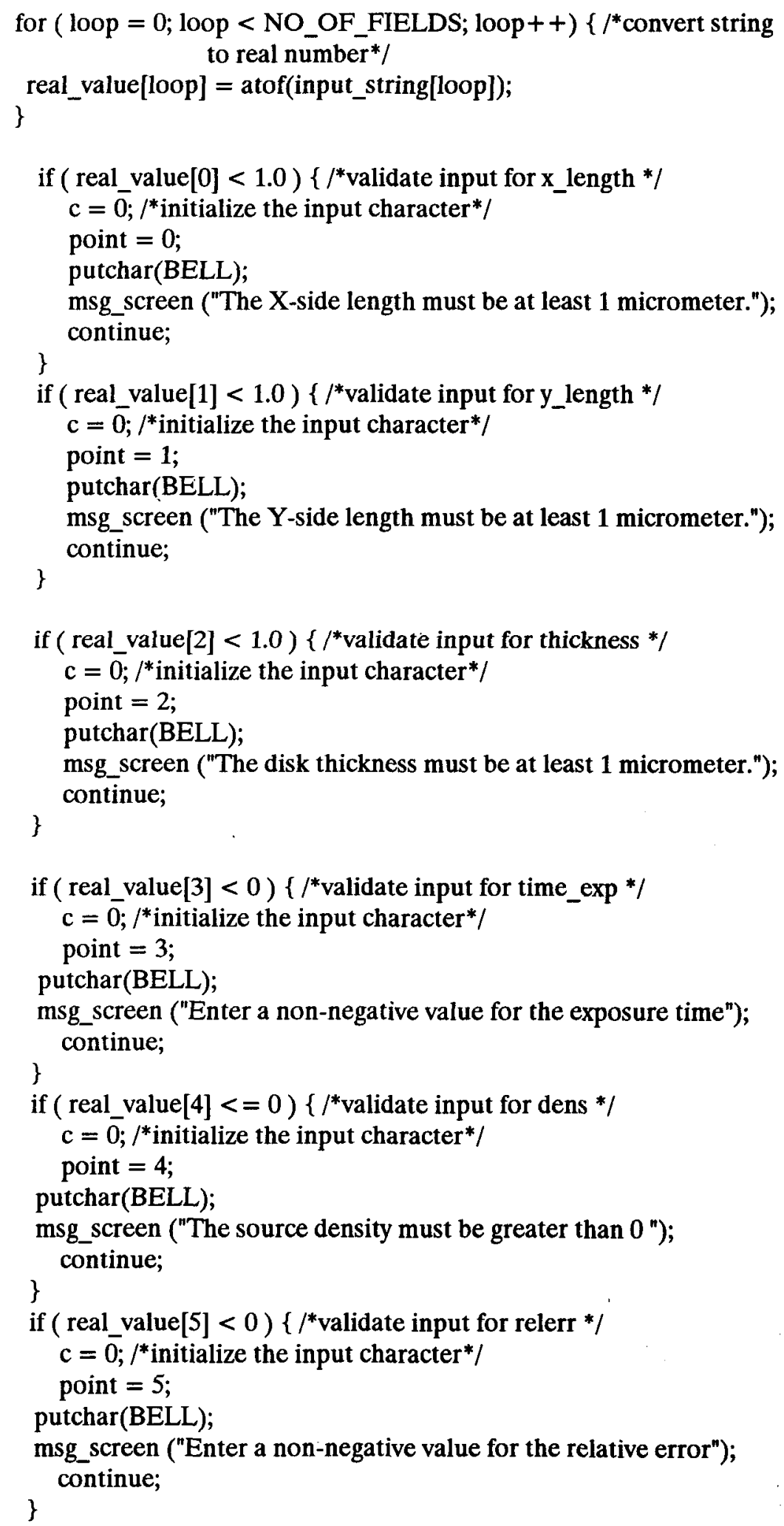



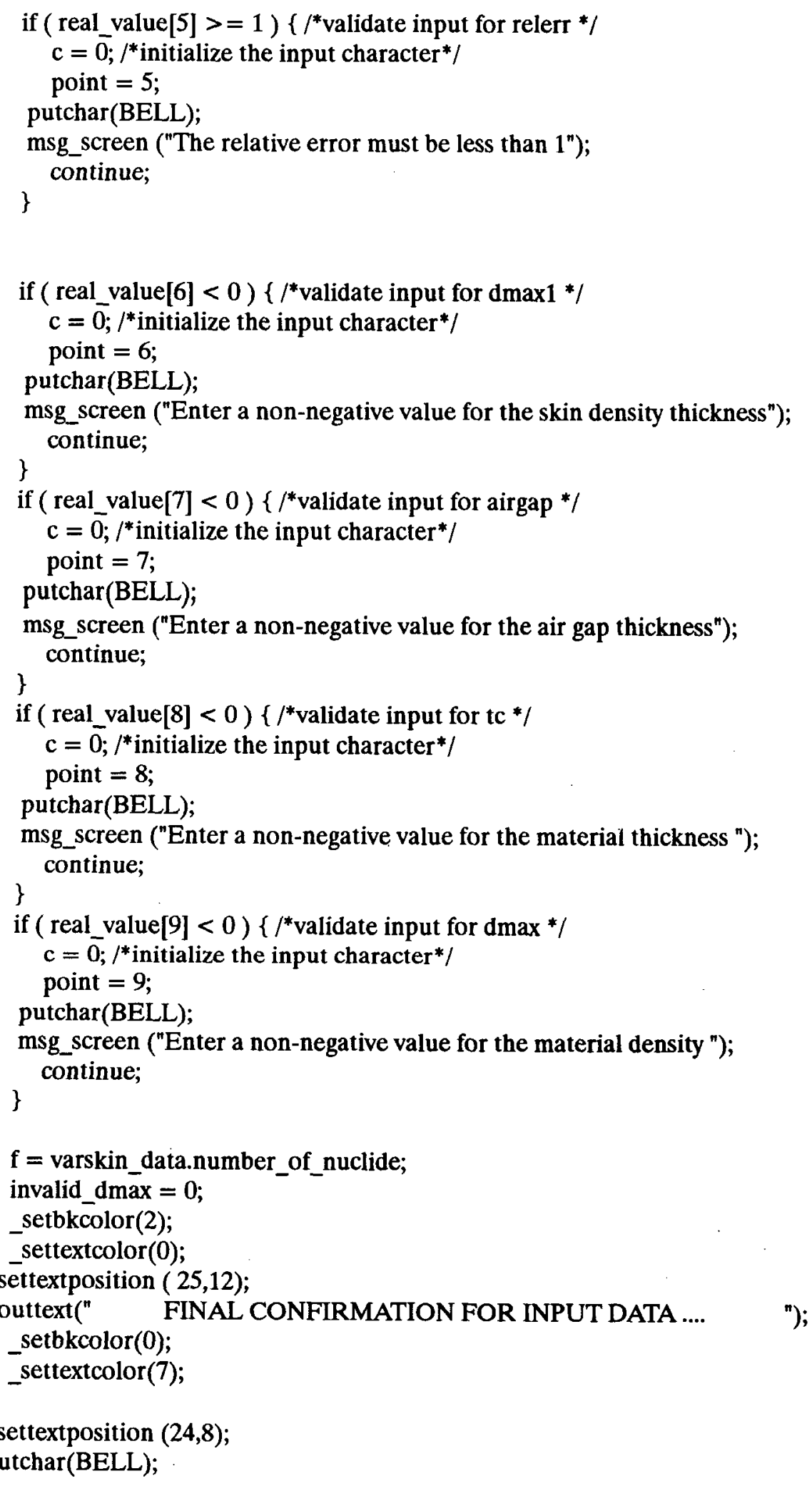


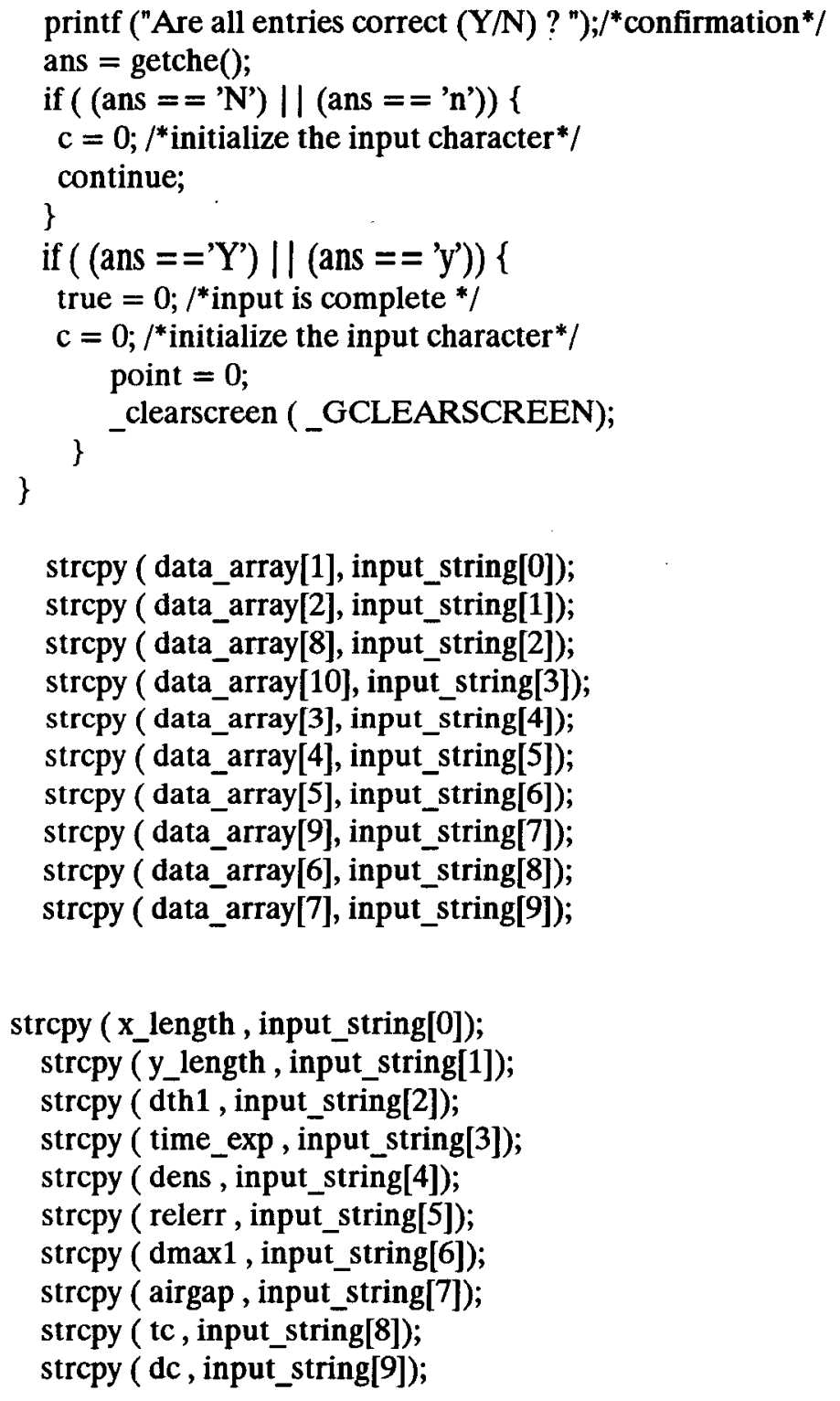




\section{PROGRAM MODULE GAMMA.C}

\#include < graph.h > \#include $<$ stdio.h $>$ \#include < math.h $>$ \#include "varglob.h" \#include "varskin.h"

\#define STRING_SIZE 9

\#define BELL 7

extern VARSKIN_DATA_TYPE varskin_data; $\quad l^{*}$ definition in varskin.h file */

extern window( int window_row1, int window_col1, int window_row2, int window_col2, char attr); $/ *$ in select 0. file $* /$

extern float geavg[6], gfac[6];

extern int selected_source_type; /*indicates sourcetype selected; decalred in src.c file*/

extern char diameter[9], dth1 [9], time_exp[9], dens[9], relerr[9], dmax1 [9],

airgap[9], tc[9], $x_{-}$length[9], y_length[9], dc[9]; char gamma_answer []$=$ "nnnnnn";

\section{gamma()}

\{

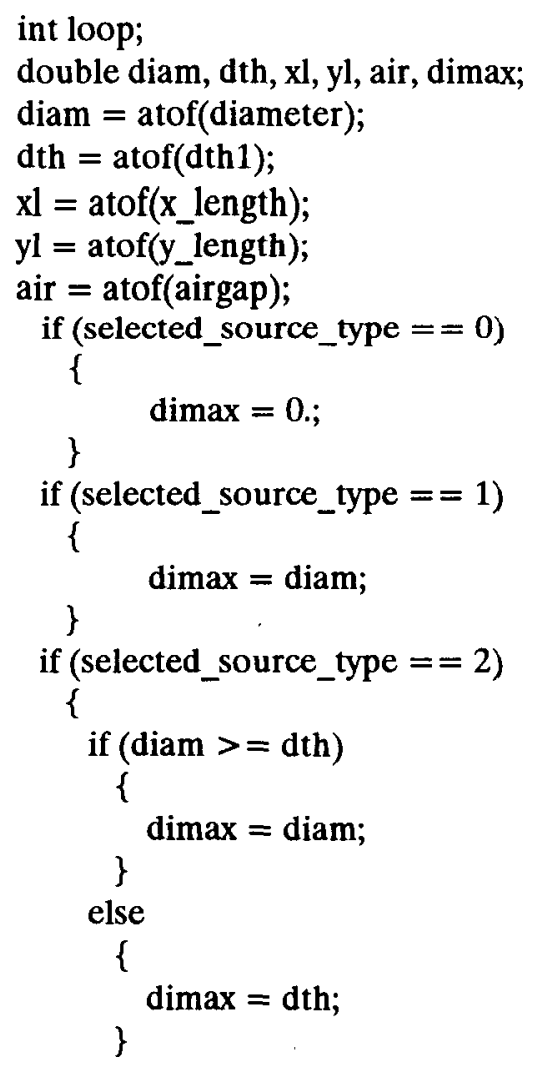


Appendix D

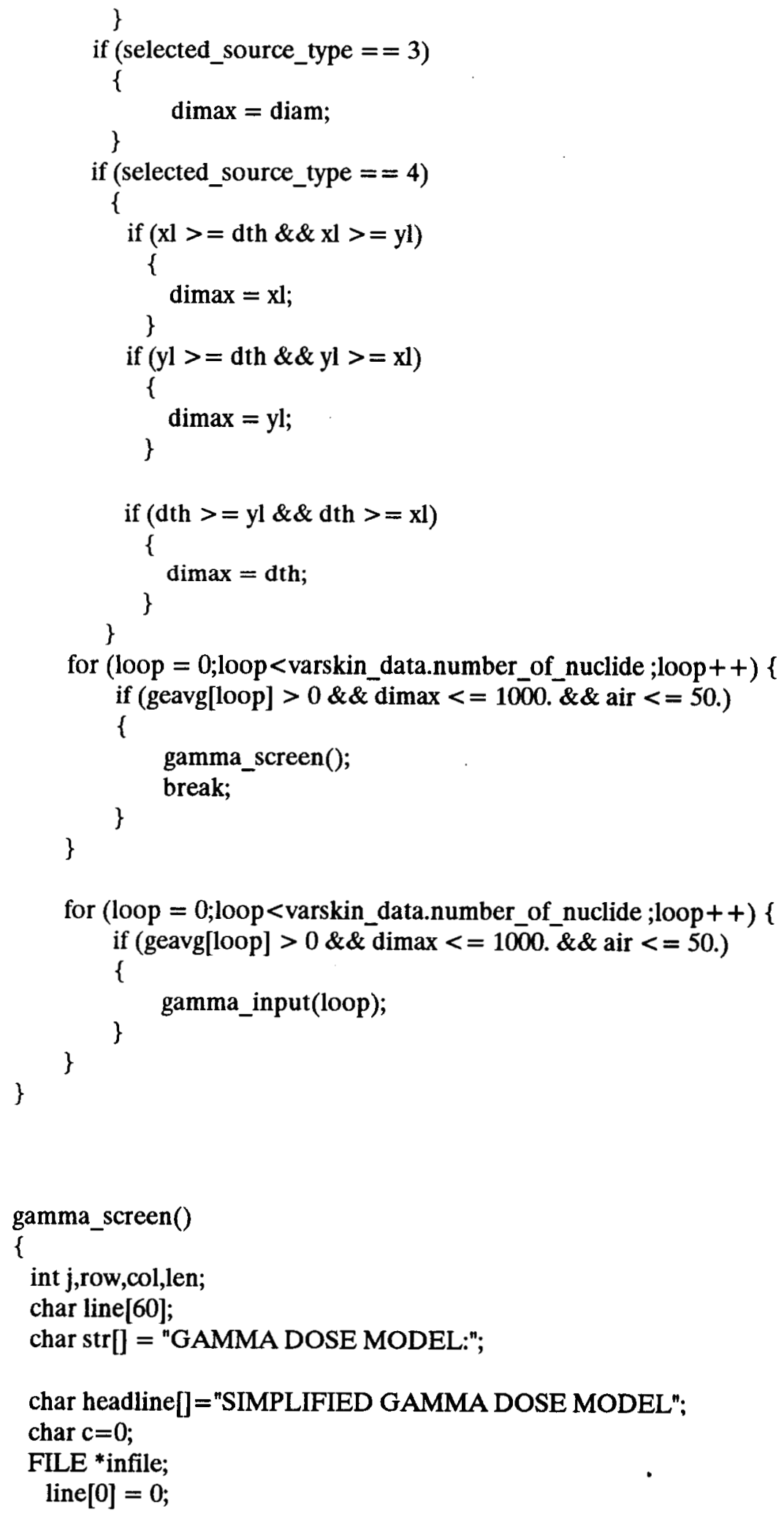




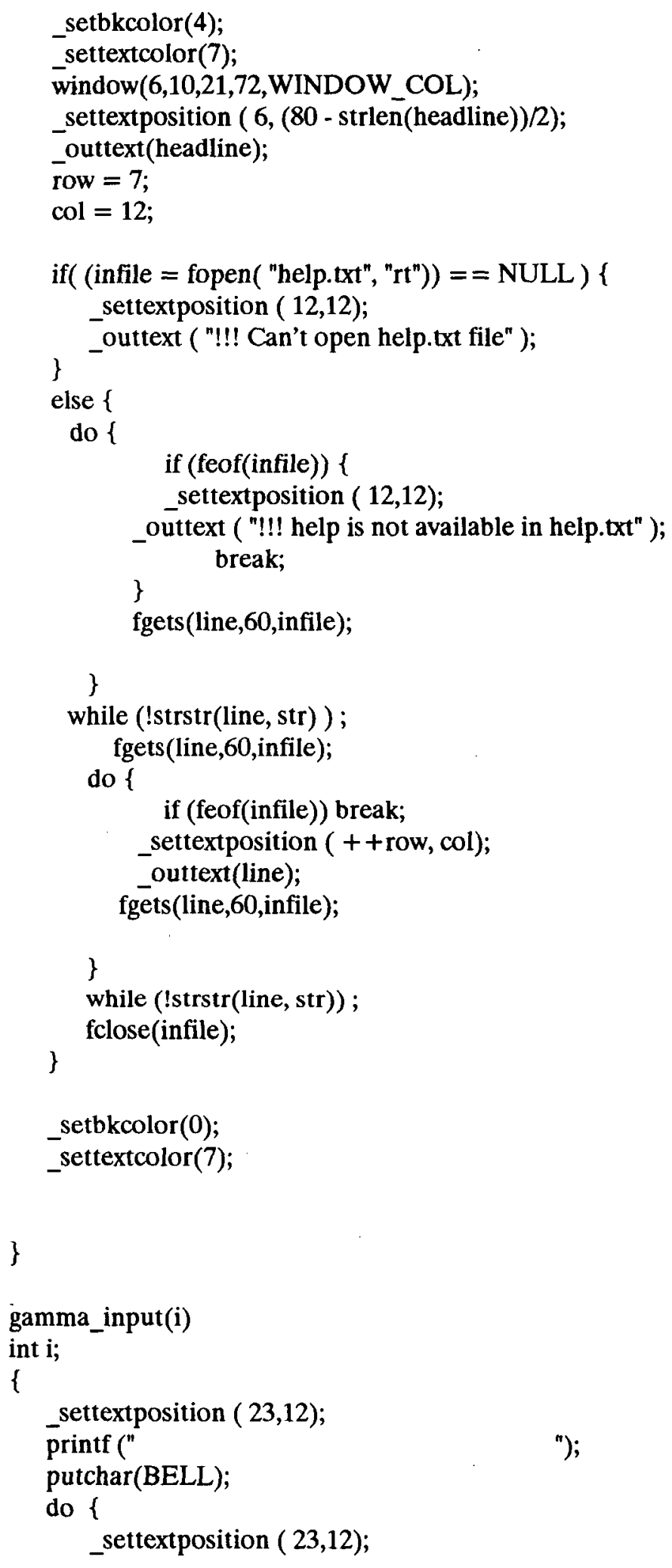


Appendix D

printf ("Do you want the gamma dose to be calculated for \%s (Y/N)? ", varskin_data.nuclide_data[i].nuclide_name);

\} gamma_answer $[\mathrm{i}]=$ getche () ;

while ( (gamma_answer[i] != 'n') \&\& (gamma_answer[i] !='N') \& \& (gamma_answer[i] ! = 'y') \&\& (gamma_answer [i] !='Y')); 


\section{PROGRAM MODULE OUTPUT.C}

\section{\#include < graph.h $>$ \\ \#include <stdio.h $>$ \\ \#include "varskin.h"}

extern VARSKIN_DATA_TYPE varskin_data; $\quad l^{*}$ definition in varskin.h file */

extern float ebeta[6], xninty[6], byield[6], fbeta[6][30];

extern float geavg[6], gfac[6];

extern char gamma_answer[6];

extern char vol_answer;

extern char data_array[12][9]; /*in dataread.c file */

char diameter[9], x_length[9], y_length[9], dth1[9], dens[9], relerr[9],

dmax 19], tc[9], dc [9], airgap [9], thskin[9], time_exp [9] ;

extern int isource $; / *$ indicates distributed source or not, declared in varmain.c file */

extern int selected_source_type ;/*indicates sourcetype selected

float ss[6];

$$
\text { declared in src.c file*/ }
$$

int geo, $\mathrm{f}=0$;

char name[6][8];

data_output()

\{

int $\mathbf{i}, \mathrm{j}, \mathrm{n}, \mathrm{c}$, loop, loc;

FILE *outfile;

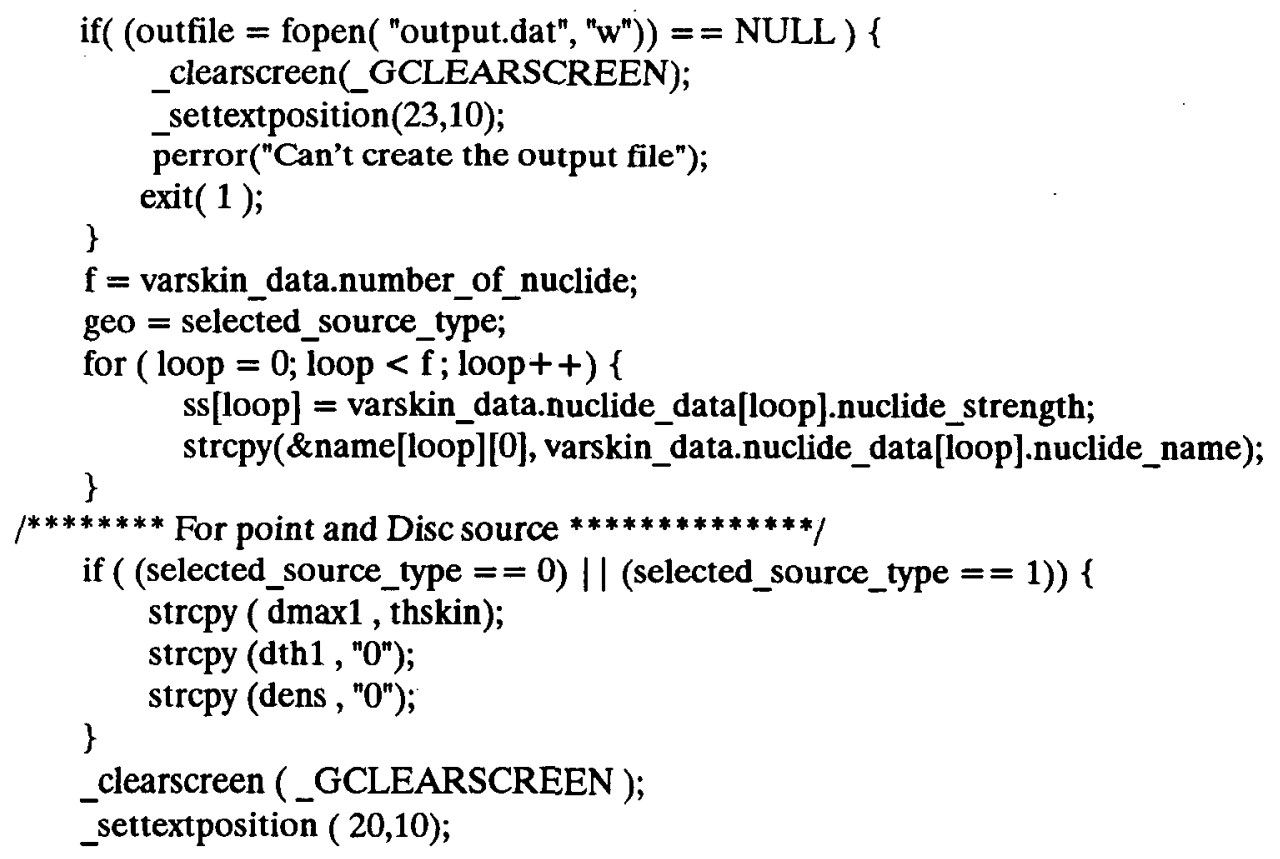




\section{Appendix D}

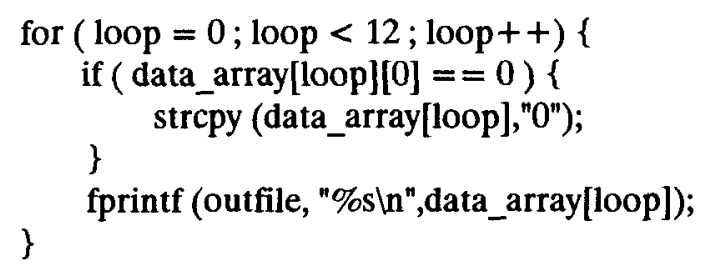




\section{PROGRAM MODULE HELP.C}

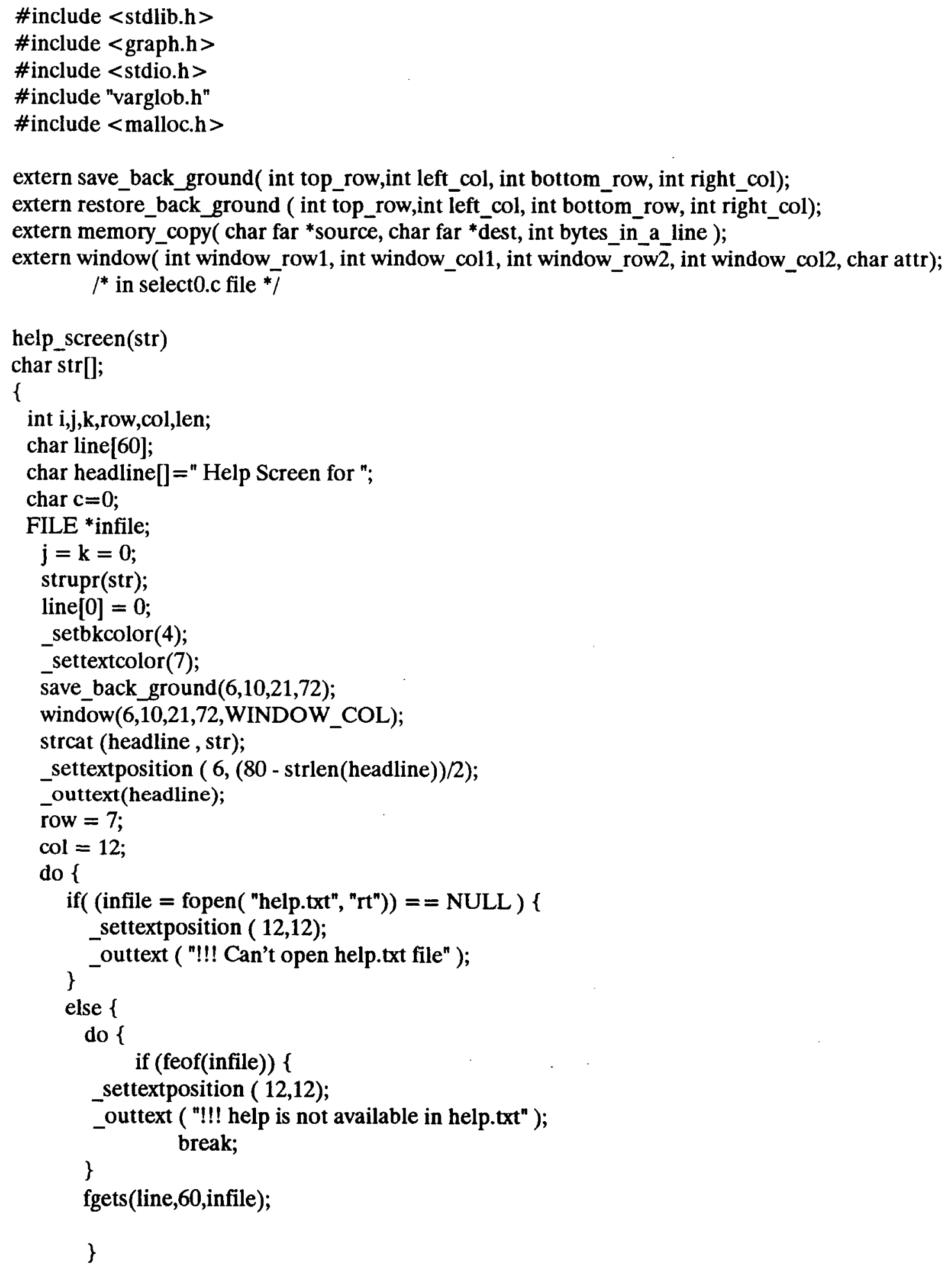




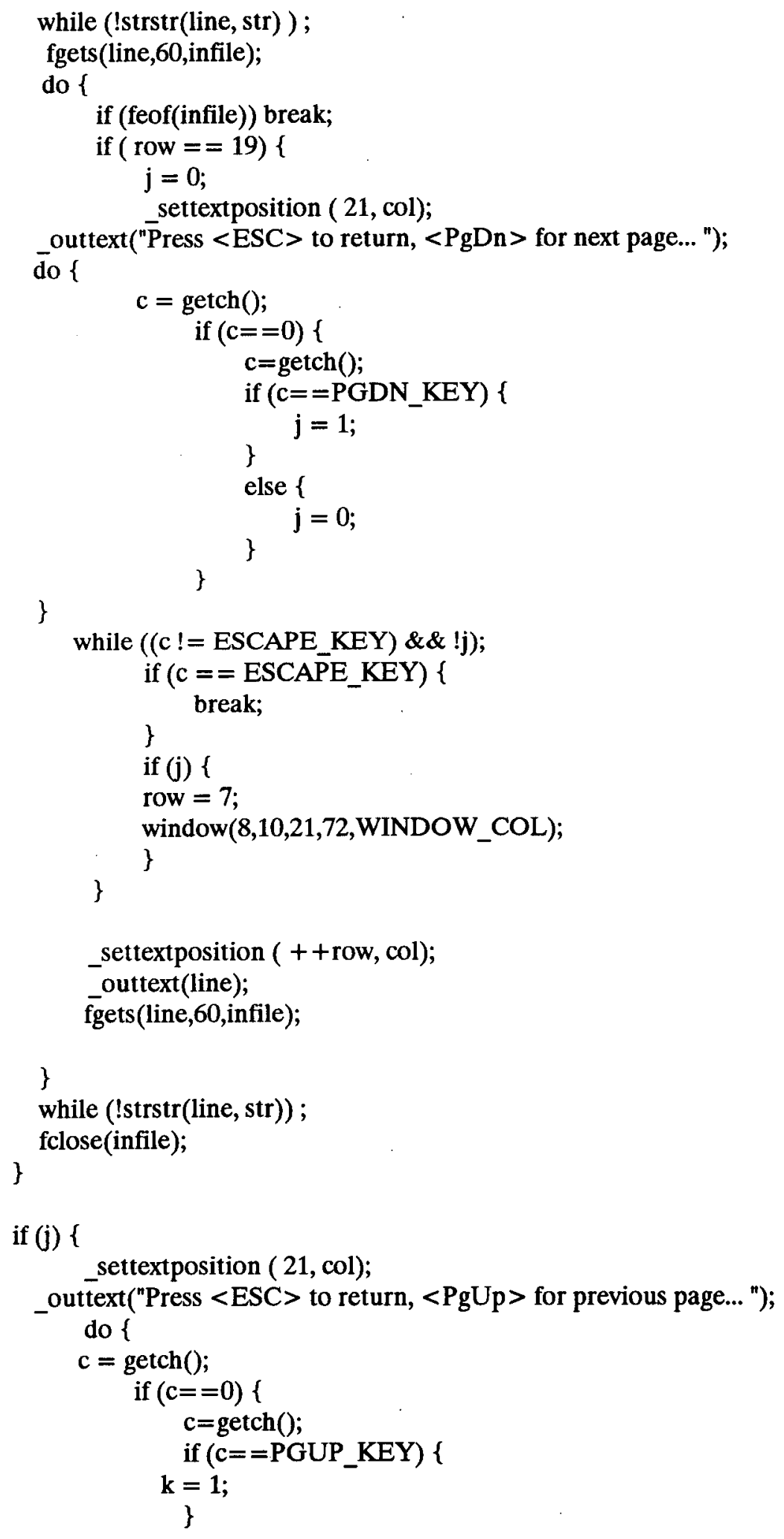




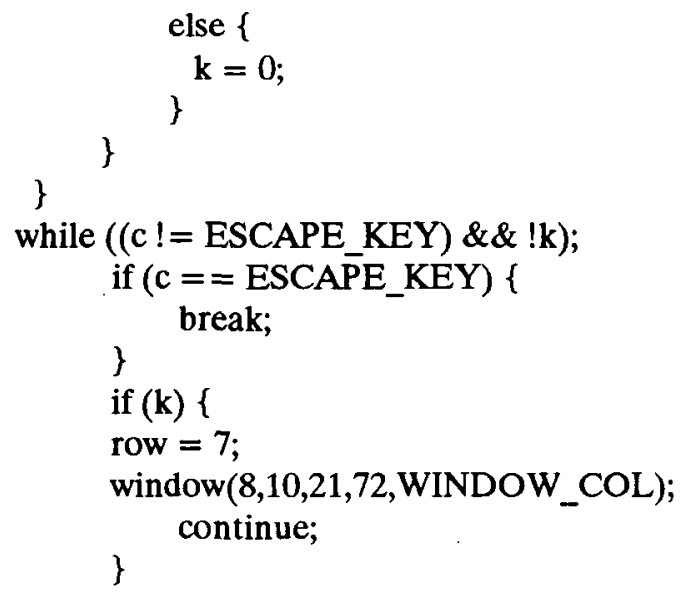

\}

_settextposition ( 21, col);

_outtext("Strike <ESC > to return ....");

while (c != ESCAPE_KEY) \{

$\mathrm{c}=\operatorname{getch}()$;

\}

break;

\}

while (1);

restore_back_ground $(6,10,21,72)$;

_setbkcolor(0);

_settextcolor(7);

\} 
APPENDIX E

TEXT OF SADDE Mod 2 CODE 


\section{APPENDIX E \\ TEXT OF SADDE Mod 2 CODE}

\section{\$LARGE}

program sadde 1

c Modified by JSD on 22 June 199, and 6 Oct 1992

dimension eb(150),spec(30,150),comp(1500),x(1000),phi(1000)

real xx90(1000),f(1000),b(150),c(150),d(150),by(30)

dimension $x r(30)$, fit(30),spec1(150),spec2(150), eele(30),yele(30)

character*1 answ

CHARACTER *6 NUCLIDE, nucnew

character*80 deck $(30,20)$,dtemp

LOGICAL*4 EXXT

c

data $x r / 0, . .01, .02, .03, .04, .05, .10, .15, .20, .25, .30, .35, .40, .45$,

1 . $50, .55, .60, .65, .70, .75, .80, .85, .90, .95,1.0,1.1,1.2,1.4,1.6,1.8 /$

c

$\mathrm{n}=150$

iwt $=1$

$\mathrm{nz}=8$

niso $=1$

ntot $=0$

byield $=0$.

emax $=0$.

write $\left({ }^{*},{ }^{*}\right)$ Please enter the isotope (or mixture) name (6 charact

\&ers)'

$\operatorname{read}(*, 1)$ nucnew

1 format(a6)

write $\left({ }^{*},{ }^{*}\right)$ ' Are multiple isotopes to be included? [y/n]'

$\operatorname{read}\left({ }^{*},{ }^{\prime}(a) '\right)$ answ

if(answ.eq.'y'.or.answ.eq.'Y')then

49 write $(*, *)$ ' Enter the total number of isotopes:'

$\operatorname{read}\left({ }^{*},{ }^{*}\right)$ niso

if(niso.gt.30)then

write $\left({ }^{*}{ }^{*}\right)$ ' The number of isotopes must be less than $30^{\prime}$

go to 49

endif

endif

$c+1$

do $502 \mathrm{j}=1$, niso

write $\left({ }^{*}, 10000\right) \mathrm{j}$ 
Appendix E

10000 format(' Data for isotope number ',i2)

write $\left({ }^{*}, 10001\right)$

10001 format $(\rho$ Enter the number of decay paths for this ',

\& 'radionuclide: '\$)

$48 \operatorname{read}\left({ }^{*},{ }^{*}\right) \bmod$

if(mod.gt.20)then

write $\left({ }^{*},{ }^{*}\right)$ ' The number of decay paths must be less than $20^{\prime}$

go to 48

endif

write $\left({ }^{*}, 10002\right)$

10002 format(' Enter the ATOMIC NUMBER of the transition (daughter)' \&'nucleus:',\$)

$\operatorname{read}\left({ }^{*},{ }^{*}\right) \mathrm{z}$

write $(*, 10003)$

10003 format(' Enter the ATOMIC MASS of the parent nucleus: '\$)

$\operatorname{read}\left({ }^{*},{ }^{*}\right) \mathrm{a}$

if(niso.gt.1)then

write $\left({ }^{*}, 10004\right)$

10004 format(' Enter the weighting factor for the radionuclide: '\$)

$\operatorname{read}\left({ }^{*}, *\right)$ wt

else

$\mathrm{wt}=1$.

endif

write(deck(j,1),'(i2,f7.1,99.3,e9.2,3i6)')mod,z,a,wt,n,iwt,nz

do $50 \mathrm{i}=2, \bmod +1$

write $\left.{ }^{*}, 10005\right) \mathrm{i}-1$

10005 format(' Decay Path Number ',i2)

write $\left({ }^{*}, 10006\right)$

10006 format $(/$ Enter the endpoint (maximum) beta energy in MeV: '\$) $\operatorname{read}\left({ }^{*},{ }^{*}\right) \mathrm{em}$

write $(*, 10007)$

10007 format $(\rho$ Enter the fractional Yield: ' $\$)$

$\operatorname{read}\left({ }^{*},{ }^{*}\right)$ yld

write $\left({ }^{*}, 10008\right)$

10008 format $(P$ Enter the forbiddenness: ',\$)

read $\left({ }^{*},{ }^{*}\right) \mathrm{nf}$

byield $=$ byield + yld ${ }^{*}$ wt

if(em.gt.emax)then

emax $=$ em

$\mathrm{jmax}=\mathbf{j}$

endif

write(deck(j,i),'(2f8.4,i6)')em,yld,nf

50 continue

by $(j)=$ byield

byield $=0$.

C 
C Add internal conversion and Auger electrons

C write $\left({ }^{*}, 10009\right)$

10009 format $(/$ Enter the number of internal conversion and Auger ', $\& \Gamma$ electrons for this radionuclide: ' $\$$ )

$\operatorname{read}\left({ }^{*},{ }^{*}\right)$ nele

ntot $=$ ntot + nele if(ntot.gt.30)then

write $\left({ }^{*}, 10011\right)$

10011 format $(\rho$ The TOTAL number of electrons in a run must be less ', \&'than 30.',/,' Please re-start Sadde Mod 2')

go to 552 endif

if(nele.eq.0)go to 502

do $502 \mathrm{i}=$ ntot-nele +1 ,ntot

6161 write $(*, 10010)$ i-ntot + nele

10010 format $(P$ Enter the energy in MeV for electron number ',i2,' :'

$\&, \$)$

$\operatorname{read}\left({ }^{*},{ }^{*}\right) \operatorname{eele}(\mathrm{i})$

if(eele(i).le.0.)then

write $\left({ }^{*}, 20001\right)$

20001 format $(P$ The electron energy must be greater than $0 . ')$

go to 6161

endif

write $\left({ }^{*}, 10012\right)$ i-ntot + nele

10012 format $(/$ Enter the fractional yield for electron number ',i2,

\& ','\$)

$\operatorname{read}\left({ }^{*}, *\right)$ yele(i)

if(yele(i).le.0.)then

write $\left({ }^{*}, 20002\right)$

20002 format $(\rho$ The yield must be greater than 0 . Restart this entry')

go to 6161

endif

byield $=$ byield + yele $(\mathrm{i})$

502 continue

445 if(answ.eq.'y'.or.answ.eq.'Y')then

c

c modify decks to account for emax

c

do $444 \mathrm{j}=1$,niso

if(j.eq.jmax)go to 444

$\operatorname{read}\left(\operatorname{deck}(\mathrm{j}, 1),{ }^{\prime}(\mathrm{i} 2, \mathbf{f 7} .1, \mathbf{f 9} .3, \mathrm{e} 9.2,3 \mathrm{i} 6)^{\prime}\right) \bmod , \mathrm{z}, \mathrm{a}, \mathrm{wt}, \mathrm{n}, \mathrm{iwt}, \mathrm{nz}$

$\bmod =\bmod +1$

write(deck(j,1),'(i2,f7.1,f9.3,e9.2,3i6)')mod,z,a,wt,n,iwt,nz

do $55 \mathrm{i}=\bmod +1,3,-1$

$\operatorname{deck}(\mathrm{j}, \mathrm{i})=\operatorname{deck}(\mathrm{j}, \mathrm{i}-1)$ 
Appendix E

$55 \quad \begin{gathered}\text { continue } \\ \text { write(deck(j,2),'(f8.4)')emax }\end{gathered}$

444 continue

c

c rank decks in order of decreasing endpoint energies

c

do $563 \mathrm{j}=1$, niso

$\operatorname{read}\left(\operatorname{deck}(\mathrm{j}, 1),{ }^{\prime}(\mathrm{i} 2, \mathrm{f} 7.1, \mathbf{f 9 . 3 , e 9 . 2 , 3 i 6 ) ' ) m o d , z , a , w t , n , i w t , n z}\right.$

if(mod.ge.2)then

561 nswap $=0$

do $562 \mathrm{i}=2$, $\bmod$

$\operatorname{read}\left(\operatorname{deck}(\mathrm{j}, \mathrm{i}),{ }^{\prime}(\mathrm{f} 8.4)^{\prime}\right) \mathrm{e} 1$

$\operatorname{read}\left(\operatorname{deck}(\mathrm{j}, \mathrm{i}+1),(\mathrm{f} 8.4)^{\prime}\right) \mathrm{e} 2$

if(e1.1t.e2)then

$\operatorname{dtemp}=\operatorname{deck}(\mathrm{j}, \mathrm{i}+1)$

$\operatorname{deck}(\mathrm{j}, \mathrm{i}+1)=\operatorname{deck}(\mathrm{j}, \mathrm{i})$

$\operatorname{deck}(\mathrm{j}, \mathrm{i})=\operatorname{dtemp}$

nswap $=1$.

endif

562 continue

if(nswap.ne.0)goto561

endif

563 continue

c

do $564 \mathrm{j}=1$,niso

call beta(j,eb,spec,deck)

byield $=$ byield + by $(j)$

564 continue

565 do $57 \mathrm{i}=1, \mathrm{n}$

do $566 \mathrm{j}=1$,niso

$\operatorname{spec} 1(i)=\operatorname{spec} 1(i)+b y(j) * \operatorname{spec}(j, i) /$ byield

566 continue

57 continue

else

c

c single isotope - reorder deck

c

byield $=$ by $(1)$

if(mod.ge.2)then

571 nswap $=0$

do $572 \mathrm{i}=2$, mod

$\operatorname{read}\left(\operatorname{deck}(1, \mathrm{i}),{ }^{\prime}(\mathbf{f 8 . 4 )})\right.$ ') 1

$\operatorname{read}\left(\operatorname{deck}(1, \mathrm{i}+1),{ }^{\prime}(\mathbf{f 8 . 4 )}) \mathrm{e} 2\right.$

if(e1.1t.e2)then

$\operatorname{dtemp}=\operatorname{deck}(1, \mathrm{i}+1)$

$\operatorname{deck}(1, \mathrm{i}+1)=\operatorname{deck}(1, \mathrm{i})$ 


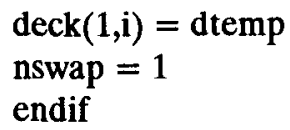

572 continue

if(nswap.ne.0)goto571

endif

c

call beta(1,eb,spec,deck)

do $477 \mathrm{i}=1,150$

$\operatorname{spec} 1(i)=\operatorname{spec}(1, i)$

477 continue

endif

C

C Monoeneretic electrons

$\mathrm{c}$ Re-order electrons by decreasing energies

1561 nswap $=0$

do $5621 \mathrm{i}=1$,ntot-1

if(eele(i).lt.eele $(i+1))$ then

tempe $=$ eele $(i+1)$

tempy $=$ yele $(i+1)$

eele $(i+1)=$ eele $(i)$

yele $(i+1)=\operatorname{yele}(i)$

eele $(i)=$ tempe

yele(i) $=$ tempy

nswap $=1$

endif

5621 continue

if(nswap.ne.0)goto1561

C

IF (ntot.gt.0)then

do $244 \mathrm{i}=1$, ntot

if(eele(i).gt.emax)then

C Generate new spectrum with larger EMAX

call spline(149,eb,spec1,b,c,d)

anorm $=$ eele $(\mathrm{i}) / \mathrm{emax}$

do $245 \mathrm{ii}=1,149$

$\mathrm{eb}(\mathrm{ii})=\mathrm{eb}(\mathrm{ii}) *$ anorm

$a=e b$ (ii)

$\operatorname{spec} 2(i i)=\operatorname{slvsp}(149, a, e b, s p e c 1, b, c, d)$

if (a.gt.emax)spec2(ii) $=0$.

if $($ spec2(ii).lt.0.)spec2(ii) $=0$.

245 continue

do $248 \mathrm{ij}=1,150$

$\operatorname{spec} 1(\mathrm{ij})=\operatorname{spec} 2(\mathrm{ij})$

$248 \operatorname{spec} 2(\mathrm{ij})=0$.

C Set new EMAX 
Appendix E

emax $=$ eele(i)

endif

$C$ Modify beta spectrum

edif $=\mathrm{eb}(10)-\mathrm{eb}(9)$

do $247 \mathrm{j}=1,149$

if $(e b(j) . g e . e e l e(i))$ then

$\operatorname{spec} 1(j)=\operatorname{spec} 1(j)+$ yele $(i) / e d i f$

go to 244

endif

if(j.eq.149)spec1(149) $=\operatorname{spec} 1(149)+$ yele(i)/edif

247 continue

244 continue

ENDIF

$249 \mathrm{~m}=149$

c

write $\left({ }^{*},{ }^{*}\right)$ ' generating beta spectrum'

c

$\mathrm{c}$ to save beta spectrum for verification

c

open(4,file='spectrum',status='unknown')

do $199 \mathrm{i}=1,150$

write $\left(4,{ }^{*}\right)$ eb(i),spec1(i)

199 continue

close (4)

c

200 call spline(m,eb,spec1,b,c,d)

c

do $250 \mathrm{i}=1,1500$

$\operatorname{comp}(\mathrm{i})=0.0$

250 continue

c

de $=\operatorname{emax} / 1500$.

eav $=0.0$

c

do $300 \mathrm{i}=1,1500$

$\mathrm{a}=\operatorname{de}^{*}$ (float(i)-0.5)

ans $=\operatorname{sivsp}(m, a, e b, \operatorname{spec} 1, b, c, d)$

if (ans.lt.0.)then

ans $=0$.

else

$\mathrm{e} 1=\mathrm{e} 1+$ ans $* \mathrm{a}$

$\mathrm{Y} 1=\mathrm{Y} 1+$ ans

endif

$300 \operatorname{comp}(\mathrm{i})=$ ans

eav $=\mathrm{e} 1 / \mathrm{y} 1$

c 


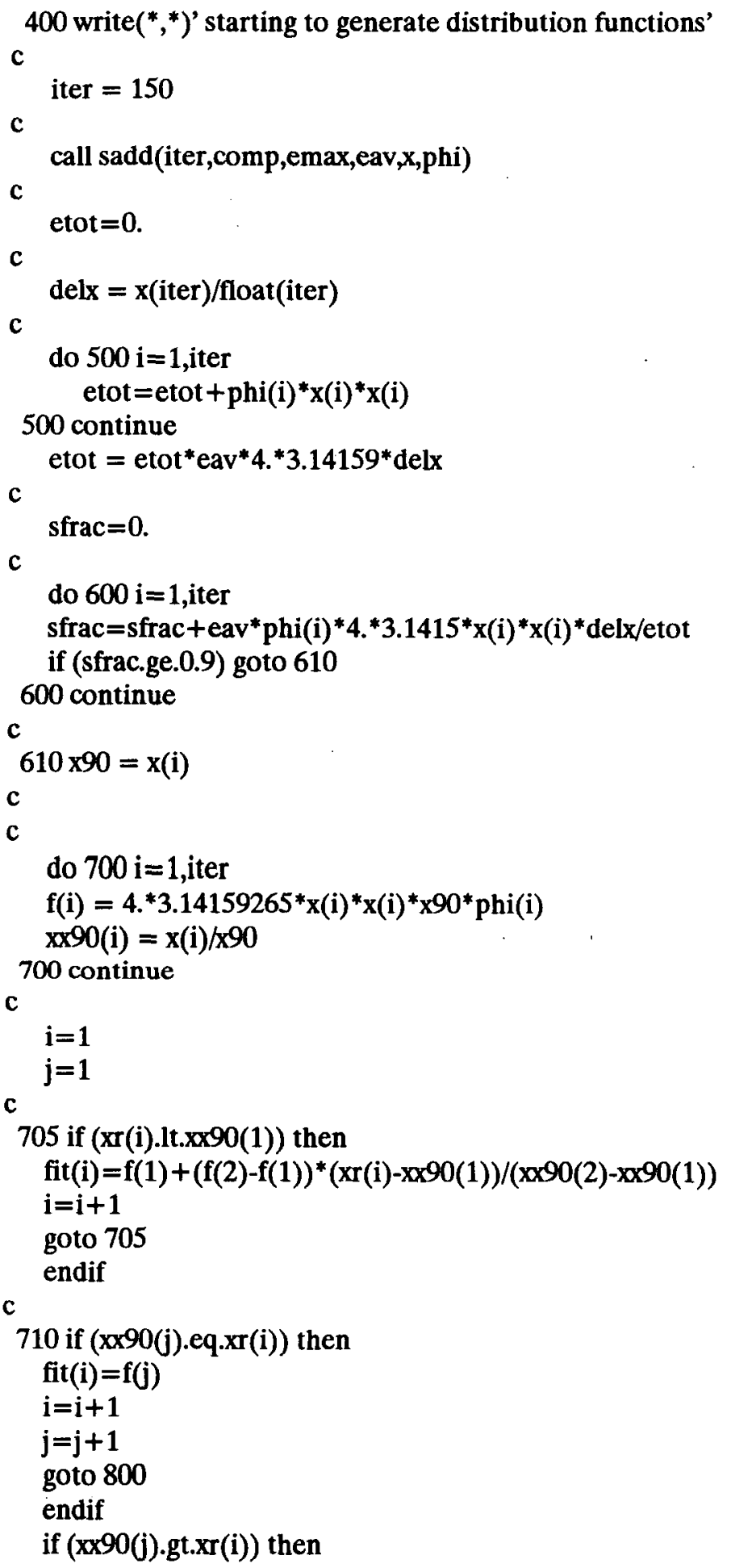


Appendix E

$$
\begin{aligned}
& \quad f i t(i)=f(j-1)+(f(j)-f(j-1))^{*}(x r(i)-x \times 90(j-1)) /(x \times 90(j)-x \times 90(j-1)) \\
& i=i+1 \\
& \text { else } \\
& j=j+1 \\
& \text { endif } \\
& 800 \text { if }(i . l e .30) \text { goto } 710
\end{aligned}
$$

c

INQUIRE(FILE='BETADATA.DAT',EXIST=EXXT)

IF ( EXXT) GOTO 549

553 WRITE(*,*)'DATA FILE BETADATA.DAT IS'

WRITE $\left({ }^{*},{ }^{*}\right)$ 'NOT ACCESIBLE'

GOTO 552

549 OPEN(21, FILE='BETADATA.DAT',STATUS='OLD')

DO 1115 INUC $=1,100$

READ(21,111)NUCLIDE

111 FORMAT(4X,A6)

IF( NUCLIDE .EQ. nucnew) then

print*, This is a duplicate nuclide.'

print*', Program Terminated'

print ${ }^{*}$, BETADATA.DAT was not altered'

close(21)

stop

endif

IF ( NUCLIDE .EQ. 'ENDOFI') then

if(inuc.gt.100)then

print*,' BETADATA.DAT contains too many isotopes'

print*', Please delete one or more isotopes and restart'

print*', this program'

go to 552

endif

backspace(21)

write $(21,111)$ nucnew

if(byield.lt.10.)then

write $(21,900)$ eav, $x 90$,byield

elseif(byield.1t.100.)then

write $(21,9001)$ eav, $x 90$,byield

else

write(21,9002)eav, x90,byield

endif

write $(21,901)($ fit $(j), j=1,30)$

write $(21,902)$

write $(21,903)$

GO TO 904

900 format $(4 x, 3 f 8.4)$

9001 format $(4 x, 3 f 8.3)$

9002 format $(4 x, 3 f 8.2)$ 


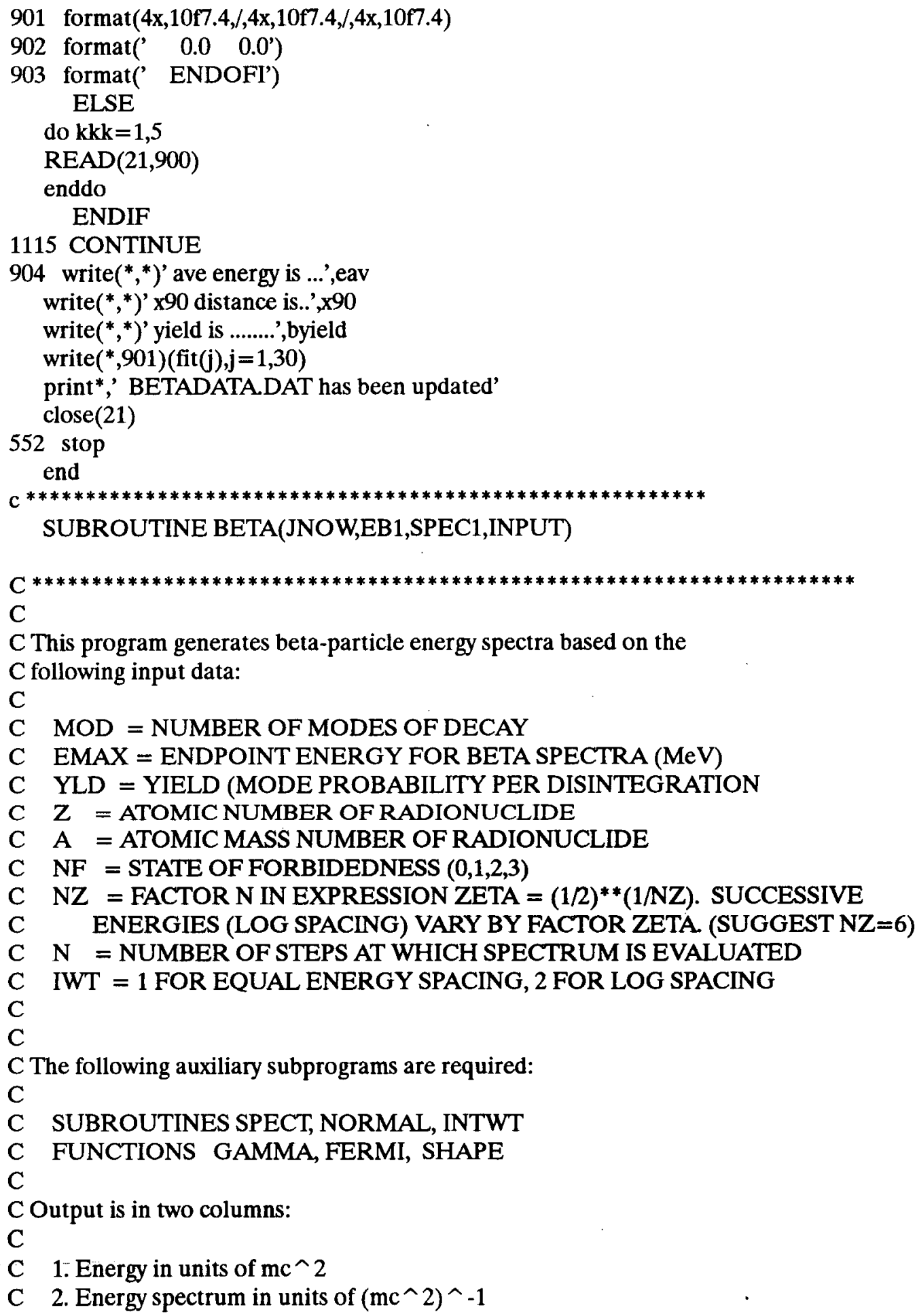


Appendix E

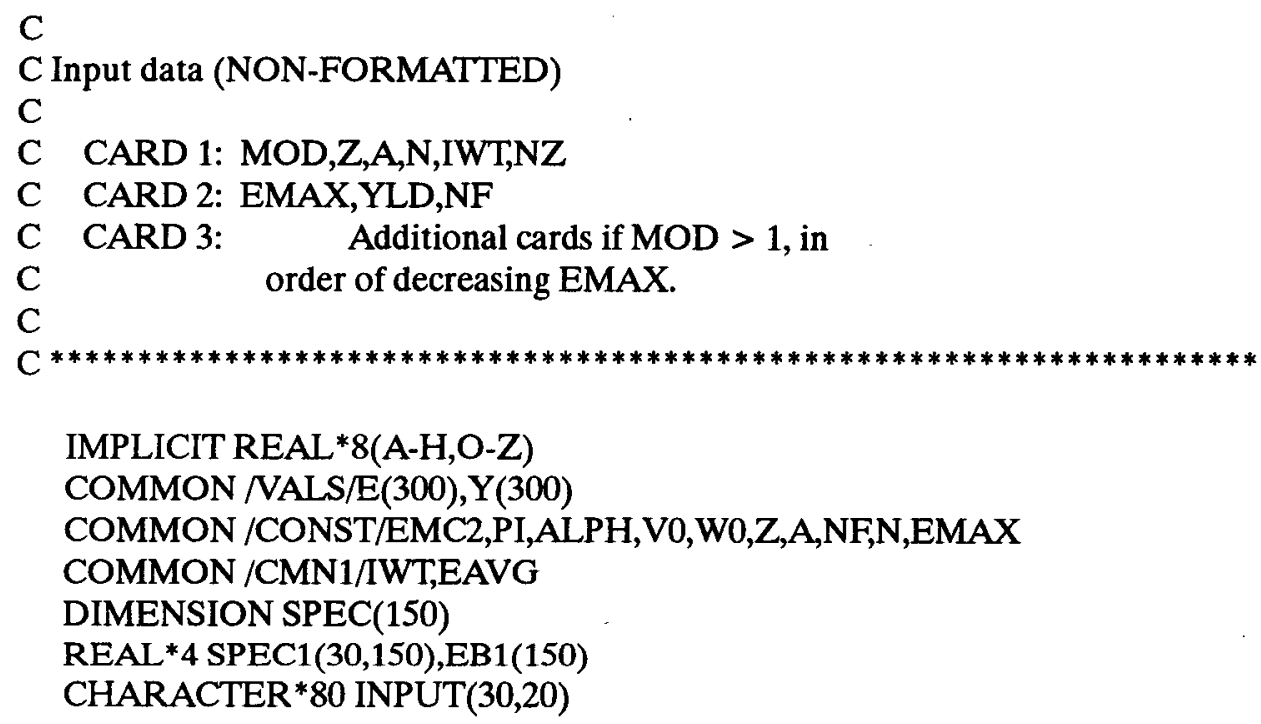


$15 \mathrm{U}=\mathrm{EMAX}^{*} \mathrm{ZT}^{* *}(\mathrm{~N}-1) / \mathrm{EMC2}$

IF (U.LT.V0) THEN

$\mathrm{N}=\mathrm{N}-1$

GO TO 15

ENDIF

NTOT $=\mathrm{N}$

DO $10 \mathrm{I}=1, \mathrm{~N}$

$\mathrm{E}(\mathrm{I})=\mathrm{EMAX}^{*} \mathrm{ZT}^{* *}(\mathrm{~N}-\mathrm{I}) / \mathrm{EMC} 2$

10 CONTINUE

ENDIF

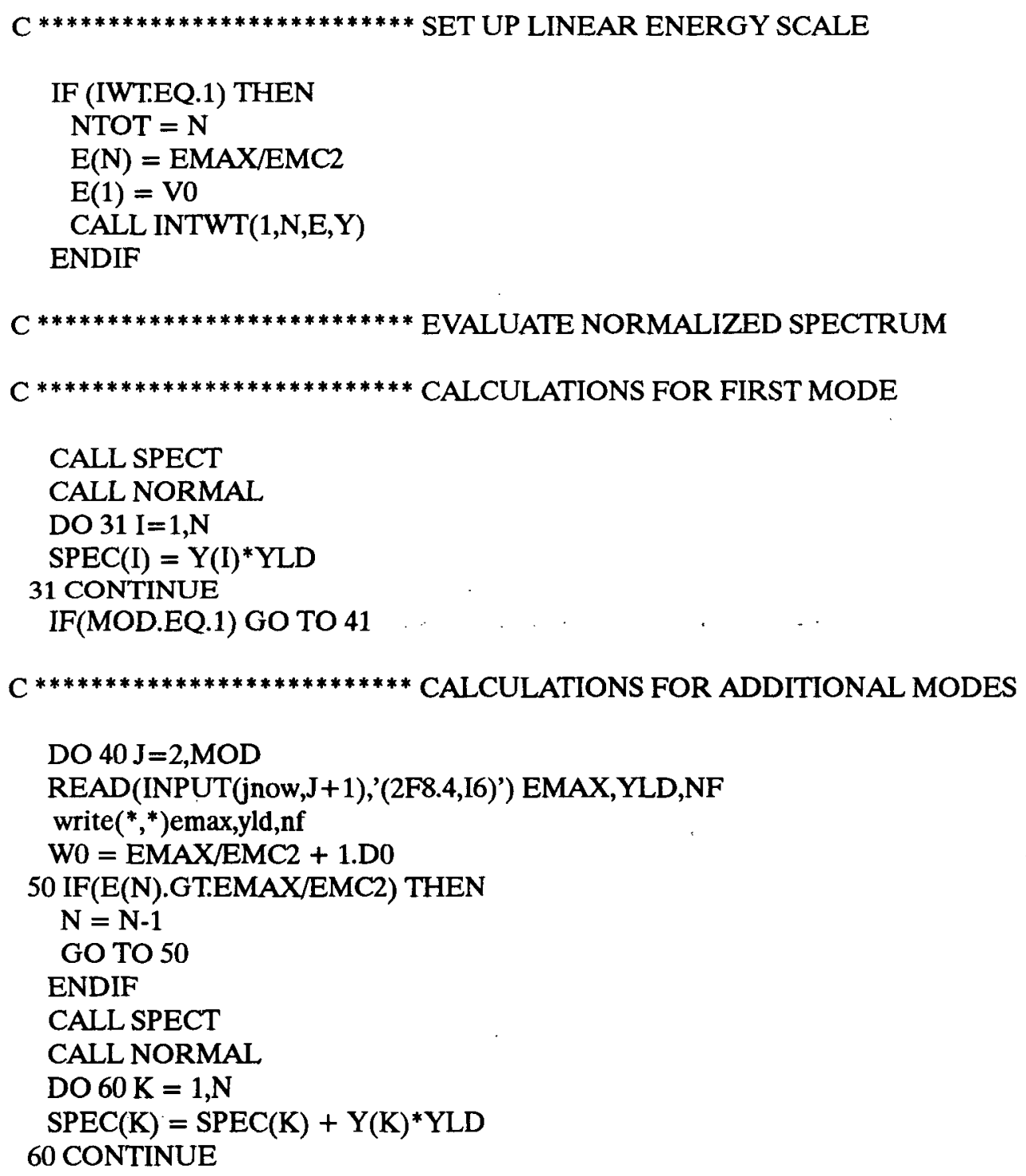


Appendix E

40 CONTINUE

41 IF(IWT.EQ.1) NSTRT $=2$

IF(IWT.EQ.2) NSTRT $=1$

80 DO 12 I=NSTRT,NTOT

$\mathrm{EB} 1(\mathrm{I}-1)=\mathrm{E}(\mathrm{I}) * \mathrm{EMC} 2$

SPEC1(jnow,I-1) $=$ SPEC(I)/EMC2

12 CONTINUE

RETURN

END

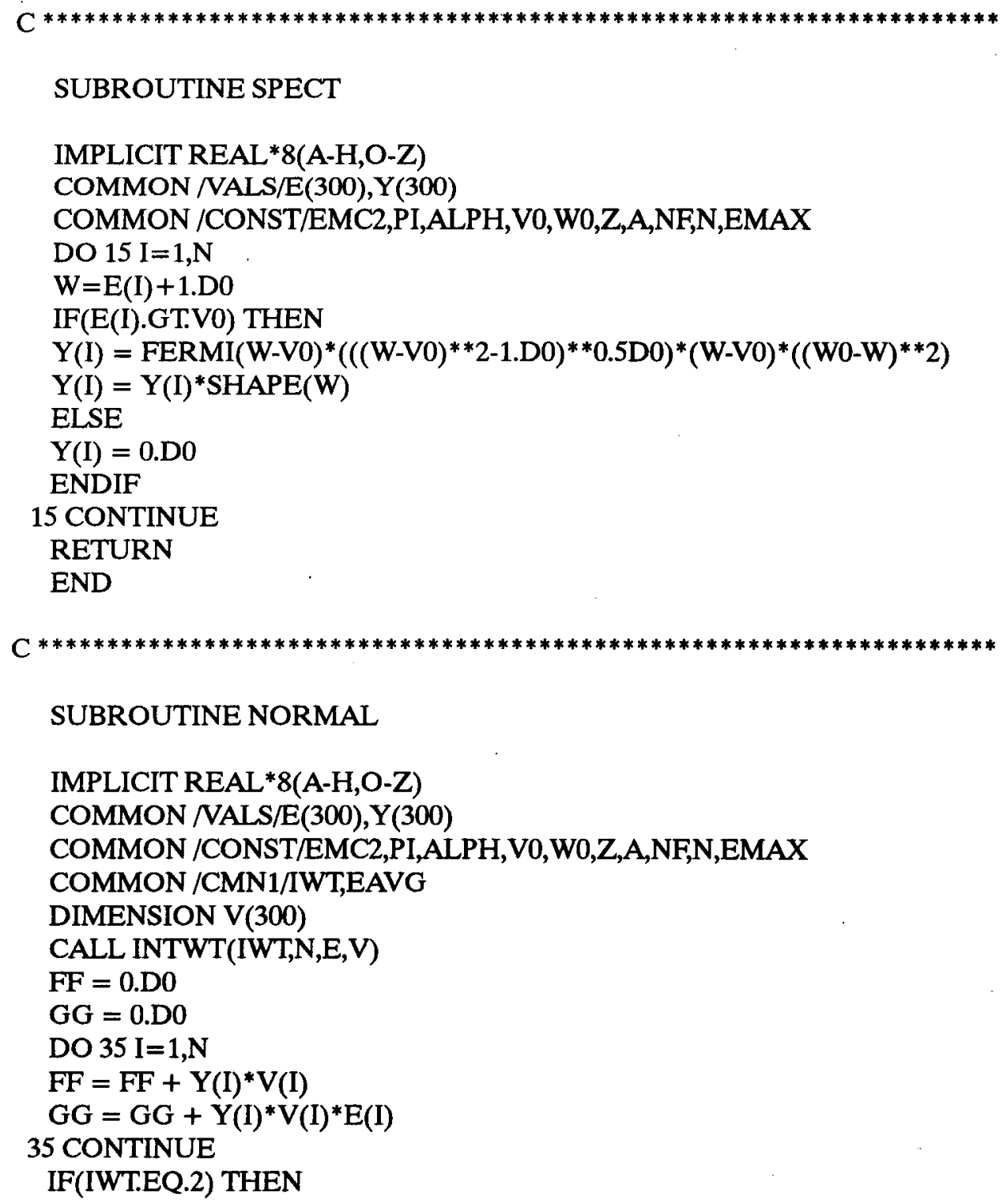




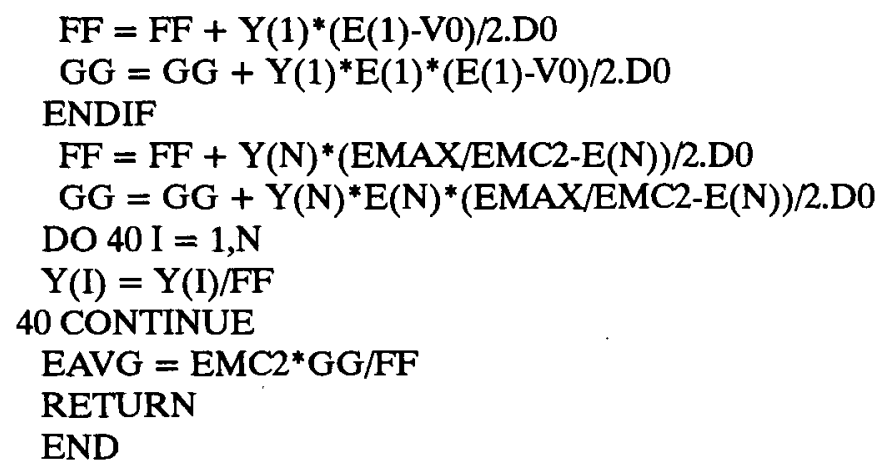

DOUBLE PRECISION FUNCTION GAMMA(X)

IMPLICIT REAL*8(A-H,O-Z)

$\mathrm{B} 1=-.577191652 \mathrm{D} 0$

$\mathrm{B} 2=.988205891 \mathrm{D} 0$

$\mathrm{B} 3=-.897056937 \mathrm{D} 0$

$\mathrm{B} 4=.918206857 \mathrm{D} 0$

$\mathrm{B} 5=-.756704078 \mathrm{D} 0$

$\mathrm{B} 6=.482199394 \mathrm{D} 0$

$\mathrm{B} 7=-.193527818 \mathrm{D} 0$

$\mathrm{B} 8=.035868343 \mathrm{D} 0$

$\mathrm{Z}=1 . \mathrm{D} 0$

10 IF(X.GE.2.D0) THEN

$\mathrm{Z}=\mathrm{Z}^{*}(\mathrm{X}-1 . \mathrm{D} 0)$

$\mathrm{X}=\mathrm{X}-1 . \mathrm{D0}$

GOTO 10

ENDIF

$\mathrm{Y}=\mathrm{X}-1 . \mathrm{D} 0$

$\mathrm{Y} 2=\mathrm{Y}^{*} \mathrm{Y}$

$\mathrm{Y} 4=\mathrm{Y} 2 * \mathrm{Y} 2$

GAMMA $=\mathrm{Z}^{*}\left(1 . \mathrm{D} 0+\mathrm{B} 1^{*} \mathrm{Y}+\mathrm{B} 2^{*} \mathrm{Y} 2+\mathrm{B} 3{ }^{*} \mathrm{Y}^{*} \mathrm{Y} 2+\mathrm{B} 4^{*} \mathrm{Y} 4+\mathrm{B} 5^{*} \mathrm{Y}^{*} \mathrm{Y} 4+\mathrm{B} 6^{*} \mathrm{Y} 4^{*} \mathrm{Y} 2+\mathrm{B} 7^{*} \mathrm{Y} 4^{*} \mathrm{Y} 2^{*} \mathrm{Y}\right.$

$\&+B 8 * Y 4 * Y 4)$

END

$\mathrm{C}$

DOUBLE PRECISION FUNCTION FERMI(W)

* Revised 2 Jul 86

IMPLICIT REAL*8(A-H,O-Z)

COMMON/CONST/EMC2,PI,ALPH,V0,W0,Z,A,NF,N,EMAX

$\mathrm{S}=\left(1 . \mathrm{D} 0-\left(\mathrm{ALPH}{ }^{*} \mathrm{Z}\right)^{* *} 2\right)^{* *} 0.5 \mathrm{D} 0-1 . \mathrm{D} 0$ 
Appendix E

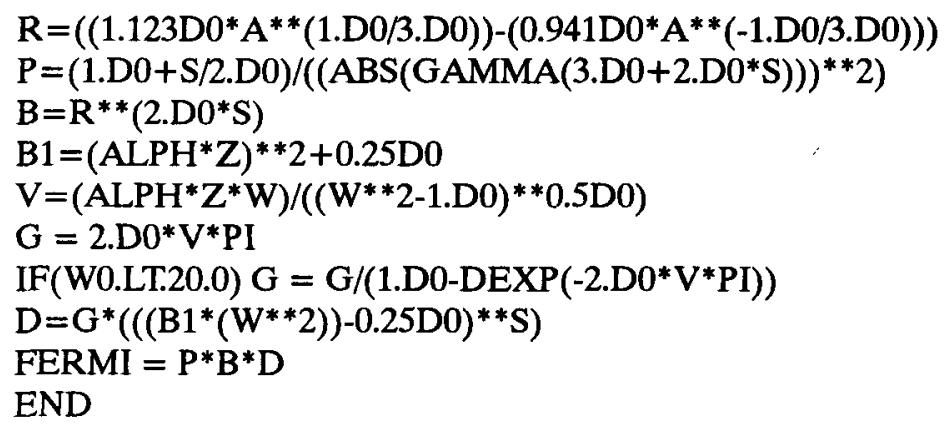

$\mathrm{C} * * * * * * * * * * * * * * * * * * * * * * * * * * * * * * * * * * * * * * * * * * * * * * * * * * * * * * * * * * * * * * * * * * * * *$

DOUBLE PRECISION FUNCTION SHAPE(W)

IMPLICIT REAL*8(A-H,O-Z)

COMMON /CONST/EMC2,PI,ALPH,V0,W0,Z,A,NF,N,EMAX

GOTO(1,2,3)NF

GOTO 10

$3 \mathrm{SN}=\left(\mathrm{W}^{* *} 2-1 . \mathrm{D} 0\right)^{* *} 3+(\mathrm{W} 0-\mathrm{W})^{* *} 6+7 . \mathrm{D} 0 *\left(\mathrm{~W}^{* *} 2-1 . \mathrm{D} 0\right)^{*}\left((\mathrm{~W} 0-\mathrm{W})^{* *} 2\right)$

$\&^{*}\left(\left(\mathrm{~W}^{* *} 2-1 . \mathrm{D} 0\right)+(\mathrm{W} 0-\mathrm{W})^{* *} 2\right)$

GO TO 14

$2 \mathrm{SN}=\left(\mathrm{W}^{* *} 2-1 . \mathrm{D} 0\right)^{* *} 2+(\mathrm{W} 0-\mathrm{W}) * * 4+(10 . \mathrm{D} 0 / 3 . \mathrm{D} 0) *\left(\mathrm{~W}^{* *} 2-1 . \mathrm{D} 0\right)^{*}((\mathrm{~W} 0-\mathrm{W}$

$\&)^{* *}$ )

GO TO 14

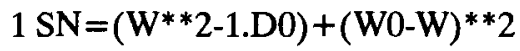

GO TO 14

$10 \mathrm{SN}=1 . \mathrm{D} 00$

$14 \mathrm{SHAPE}=\mathrm{SN}$

END

$\mathrm{C}$

SUBROUTINE INTWT(IWT,NWT,WTAB,WATES)

C IWT $=1$,EQUALLY SPACED POINTS

C IWT $=2$,SUCCESSIVELY MULTIPLICATED SPACED POINTS

C NWT---NUMBER OF ENTRIES

C WTAB---ABSCISSA LIST FOR WHICH INTEGRATION WEIGHTS ARE DETERMINED

C WATES--LIST OF INTEGRATION WEIGHTS TO BE DETERMINED

IMPLICIT REAL*8(A-H,O-Z)

DIMENSION WTAB(300),WATES(300)

WTA $=$ NWT

$W T A B 1=W T A B(1)$

WTABN $=$ WTAB $($ NWT $)$

IF(NWT-2)10,20,20 


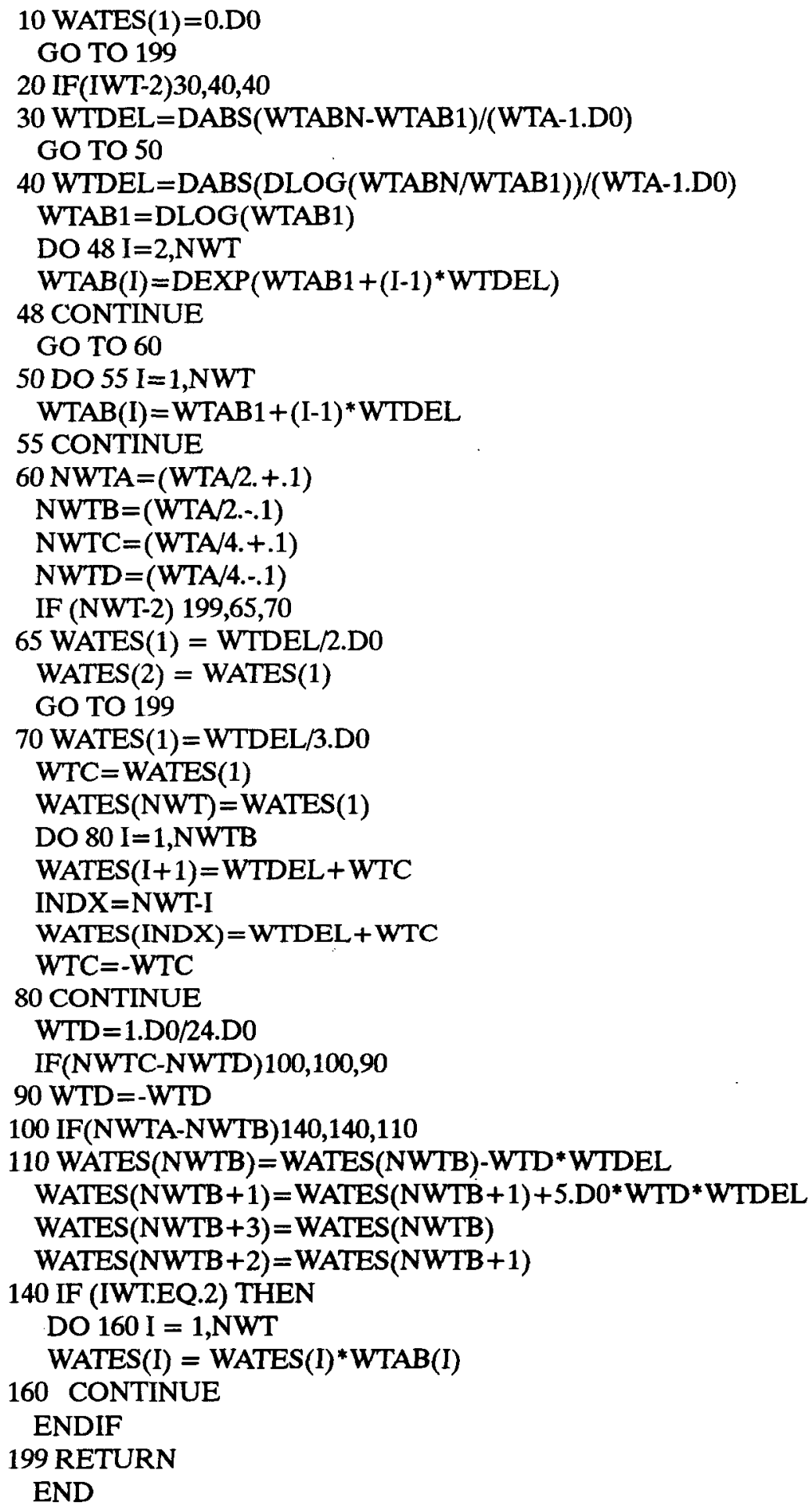


Appendix E

C

$\mathrm{C}^{* * * * * * * * * * * * * * * * * * * * * * * * * * * * * * * * * * * * * * * * * * * * * * * * * * * * * * * * * * * * * * * * * * * * * * * * * *}$

SUBROUTINE SPLINE(M,X,Y,B,C,D)

INTEGER N,M

C

REAL X(M),Y(M),B(M),C(M),D(M)

$\mathrm{N}=\mathrm{M}$
$\mathrm{NM} 1=\mathrm{N}-1$
$\mathrm{IF}(\mathrm{N} . L T .2)$ RETURN
IF(N.LT.3) GO TO 50

C

$\mathrm{D}(1)=\mathrm{X}(2) \cdot \mathrm{X}(1)$

$\mathrm{C}(2)=(\mathrm{Y}(2)-\mathrm{Y}(1)) / \mathrm{D}(1)$

DO $10 \mathrm{I}=2$,NM1

$\mathrm{D}(\mathrm{I})=\mathrm{X}(\mathrm{I}+1)-\mathrm{X}(\mathrm{I})$

$\mathrm{B}(\mathrm{I})=2 . *(\mathrm{D}(\mathrm{I}-1)+\mathrm{D}(\mathrm{I}))$

$\mathrm{C}(\mathrm{I}+1)=(\mathrm{Y}(\mathrm{I}+1)-\mathrm{Y}(\mathrm{I})) / \mathrm{D}(\mathrm{I})$

$\mathrm{C}(\mathrm{I})=\mathrm{C}(\mathrm{I}+1) \cdot \mathrm{C}(\mathrm{I})$

10 CONTINUE

C

$B(1)=-1 . * D(1)$

$\mathrm{B}(\mathrm{N})=-1 .{ }^{*} \mathrm{D}(\mathrm{N}-1)$

$C(1)=0$.

$\mathrm{C}(\mathrm{N})=0$.

IF (N.EQ.3) GO TO 15

$\mathrm{C}(1)=\mathrm{C}(3) /(\mathrm{X}(4)-\mathrm{X}(2))-\mathrm{C}(2) /(\mathrm{X}(3)-\mathrm{X}(1))$

$\mathrm{C}(\mathrm{N})=\mathrm{C}(\mathrm{N}-1) /(\mathrm{X}(\mathrm{N})-\mathrm{X}(\mathrm{N}-2))-\mathrm{C}(\mathrm{N}-2) /(\mathrm{X}(\mathrm{N}-1)-\mathrm{X}(\mathrm{N}-3))$

$\mathrm{C}(1)=\mathrm{C}(1)^{*} \mathrm{D}(1)^{* *} 2 /(\mathrm{X}(4)-\mathrm{X}(1))$

C

$\mathrm{C}(\mathrm{N})=-\mathrm{C}(\mathrm{N}) * \mathrm{D}(\mathrm{N}-1) * * 2 /(\mathrm{X}(\mathrm{N})-\mathrm{X}(\mathrm{N}-3))$

15 DO $20 \mathrm{I}=2, \mathrm{~N}$

$\mathrm{T}=\mathrm{D}(\mathrm{I}-1) / \mathrm{B}(\mathrm{I}-1)$

$\mathrm{B}(\mathrm{I})=\mathrm{B}(\mathrm{I})-\mathrm{T}^{*} \mathrm{D}(\mathrm{I}-1)$

$\mathrm{C}(\mathrm{I})=\mathrm{C}(\mathrm{I})-\mathrm{T}^{*} \mathrm{C}(\mathrm{I}-1)$

20 CONTINUE

C

$\mathrm{C}(\mathrm{N})=\mathrm{C}(\mathrm{N}) / \mathrm{B}(\mathrm{N})$

DO $30 \mathrm{IB}=1$,NM1

$\mathrm{I}=\mathrm{N}$-IB

$\mathrm{C}(\mathrm{I})=(\mathrm{C}(\mathrm{I})-\mathrm{D}(\mathrm{I}) * \mathrm{C}(\mathrm{I}+1)) / \mathrm{B}(\mathrm{I})$

30 CONTINUE

C

$$
\begin{aligned}
& \mathrm{B}(\mathrm{N})=(\mathrm{Y}(\mathrm{N})-\mathrm{Y}(\mathrm{NM} 1)) / \mathrm{D}(\mathrm{NM} 1)+\mathrm{D}(\mathrm{NM} 1)^{*}\left(\mathrm{C}(\mathrm{NM} 1)+2 .{ }^{*} \mathrm{C}(\mathrm{N})\right) \\
& \mathrm{DO} 40 \mathrm{I}=1, \mathrm{NM} 1 \\
& \mathrm{~B}(\mathrm{I})=(\mathrm{Y}(\mathrm{I}+1)-\mathrm{Y}(\mathrm{I})) / \mathrm{D}(\mathrm{I})-\mathrm{D}(\mathrm{I})^{*}\left(\mathrm{C}(\mathrm{I}+1)+2 .{ }^{*} \mathrm{C}(\mathrm{I})\right) \\
& \mathrm{D}(\mathrm{I})=(\mathrm{C}(\mathrm{I}+1)-\mathrm{C}(\mathrm{I})) / \mathrm{D}(\mathrm{I})
\end{aligned}
$$




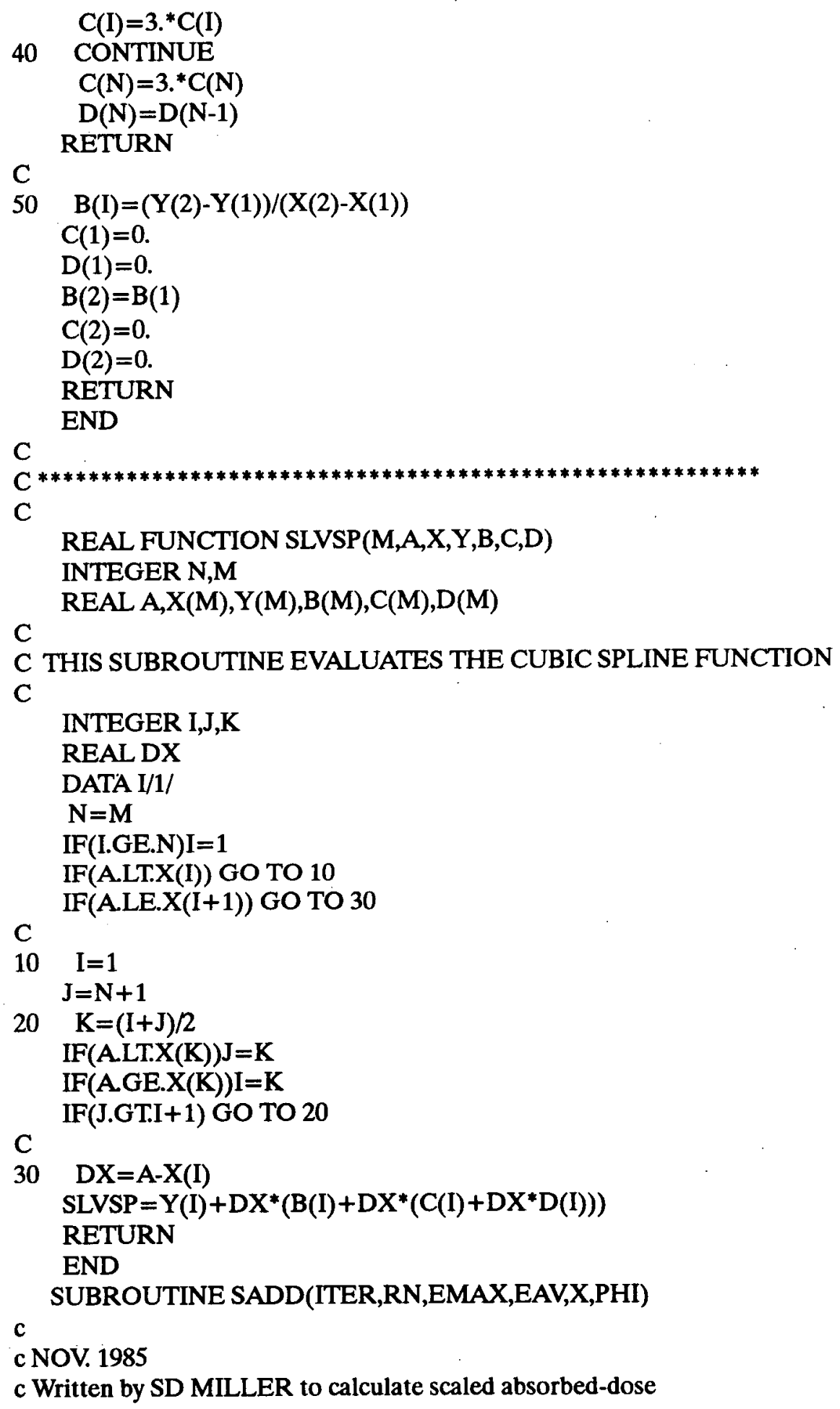


Appendix E

c distributons for beta spectra.

c

c

c Modified Dec. 1987 by SD MILLER to work with betacomp.f

c routine for complex beta emmitters.

c

c

dimension $\mathrm{rn}(1500)$, temp(1000),X(1000),PHI(1000)

dimension range $(40)$,energy $(40)$

c

data energy $/ .010, .015, .020,025, .030, .035, .040, .045$,

$1.050, .055, .060, .065, .070, .075, .080, .085, .090, .095, .10$,

$2.15, .20, .25, .30, .35, .40, .45, .50, .60, .70, .80, .90,1.00$,

3 1.5,2.0,3.0,4.0,5.0,6.0,7.0,8.0/

c

data range $/ .00024, .00050, .00083, .00124, .00171, .00225$,

1 .00285,.00351,.00422,.00499,.00580,.00667,.00759,.00855,

2 .00956,.0106,.0117,.0128,.0140,.0276,.0440,.0625,.0826,

3 .104,.126,.150,.174,.223,.273,.325,.377,.430,.696,.961,

4 1.49,2.00,2.50,2.99,3.47,3.95/

c

c Compute maximum electron range based on maximum beta energy

c

$\mathrm{jj}=1$

c

5 if (emax.lt.energy(1)) then

$x \max =$ emax $^{*}$ range(1)/energy(1)

goto 100

endif

c

10 if (energy(jj).eq.emax) then $\mathrm{xmax}=$ range $(\mathrm{jj})$

$\mathrm{jj}=\mathrm{jj}+1$

goto 100

endif

if (energy(jj).gt.emax) then

$x \max =$ range $(\mathrm{jj}-1)+($ range $(\mathrm{jj})$-range $(\mathrm{jj}-1))$

1 *(emax-energy(jj-1))/(energy(jj)-energy(jj-1)) goto 100

else

$\mathrm{jj}=\mathrm{jj}+1$

endif

goto 10

100 continue

c

delx $=\operatorname{xmax} /$ float(iter) 


$$
\text { dele }=\operatorname{emax} / \text { float(iter) }
$$

c c step through each energy in the beta spectra until EMAX.

c

do $199 \mathrm{i}=1$,iter

$199 \operatorname{temp}(\mathrm{i})=0$.

c

c pick an energy

c write $\left({ }^{*}, *\right)$

c do $299 \mathrm{k}=1$, iter

c $\mathrm{e}=\operatorname{dele}^{*}($ float $(k)-0.5)$

prob $=0$. do $298 \mathrm{~m}=1,10$ prob $=\operatorname{prob}+\operatorname{rn}((k-1) * 10+m)$

298 continue prob $=0.1 *$ prob

c c normalize the phi function before use

c

write $\left({ }^{*}, 398\right) k$,iter

398 format('+ on iteration',i4,' of',i4) sum $=0$.

c

do $399 \mathrm{i}=1$, iter $\mathrm{xs}=$ float $(\mathrm{i}) * \operatorname{del} \mathrm{x}$

c

$$
\begin{aligned}
& \text { imax }=i \\
& \text { adder }=\text { spens(e,xs) }
\end{aligned}
$$

if(adder.eq.0.0)then

goto 400

else

c

sum $=$ sum + adder ${ }^{*}$ delx $* 3.14159^{*} 4 .{ }^{*} x s^{*} x s$

endif

c

399 continue

c

400 if (sum.ne.0.) then

do $499 \mathrm{i}=1$, imax

xs $=$ float $(\mathbf{i}) * \operatorname{del} x$

temp $(i)=\operatorname{temp}(i)+$ dele $^{*}$ spens $(e, x s)^{*}$ prob*e/sum $^{*}$

499 continue

endif 
Appendix E

c

299 continue

c

do $599 \mathrm{i}=1$,iter

$x(i)=$ float $(\mathbf{i}) *$ del $x$

phi(i) $=$ temp(i)/eav

599 continue

c

c

return

end

c

$\mathrm{c} * * * * * * * * * * * * * * * * * * * * * * * * * * * * * * * * * * * * * * * * * * * * * * * * * * * * * * * * *$

c

$\mathrm{C}$

REAL FUNCTION SPENS(ENERGY,DISTANCE)

C THIS FUNCTION CALCULATES PHI OF A MONOENERGETIC ELECTRON USING DATA

C FROM THE SPENSER TABLES. PHI IS THE FRACTION OF THE TOTAL ENERGY

C DEPOSITED AT "DISTANCE" PER UNIT MASS. PHI IS CALCULATED KNOWING THE

C INITIAL ELECTON ENERGY AND THE DISTANCE FROM THE SOURCE TO THE DOSE

C POINT.

C WRITTEN BY WD REECE DEC 1987. Modified by JS Durham October 1991.

$\mathrm{C}$

DIMENSION E(20),FX90(13),F(13,20)

DIMENSION EUP(20),X90T(20)

C

DIMENSION B(13,20),C(13,20),D(13,20),B1(20),C1(20),D1(20)

C

COMMON /COMSPLI/ B,C,D,B1,C1,D1

DATA E/4.,2.,1.,0.7,0.4,0.2,0.1,0.05,0.03,0.025,0.02,0.015,

$\& 0.01,0.005,0.002,0.001,0.0005,0.0002,0.0001,0.00005 /$

DATA EUP/0.,0.0001,0.0002,0.0005,0.001,0.002,0.005,

\&0.010,0.015,0.02,0.025,0.03,0.05,.1,.2,.4,7,1.,2.,4./.

DATA X90T/0.,.0000003,.0000005,.0000018,0.0000049,0.000016,

$\& 0.000071,0.00021,0.00044,0.00075,0.00107,0.00140,0.00318,0.0106$,

$\mathrm{C}$ $\& 0.0334,0.096, .207, .328, .74,1.57 /$

DATA FX90/0.,.1,.2,.3,.4,.5,.6,.7,.8,.9,1.,1.1,1.15/

DATA F/0.75,.75,.76,.78,.80,.83,.89,.99,1.09,1.13,.97,.45,.12,

$\& 0.68, .69, .71, .74, .78, .84,0.92,1.04,1.16,1.20,1.00, .47, .18$,

$\& 0.61, .62, .65, .70, .76, .85,0.95,1.08,1.22,1.29,1.03, .49, .20$,

$\& 0.57, .59, .62, .68, .75, .85,0.96,1.10,1.25,1.31,1.04, .50, .21$,

$\& 0.52, .54, .58, .65, .73, .84,0.98,1.13,1.30,1.37,1.06, .51, .22$,

$\& 0.48, .50, .55, .62, .72, .84,1.00,1.20,1.35,1.39,1.09, .51, .22$,

$\& 0.45, .47, .53, .61, .71, .85,1.01,1.19,1.37,1.42,1.11, .50, .22$,

$\& 0.43, .45, .51, .59, .71, .86,1.03,1.21,1.37,1.41,1.13, .49, .20$, 


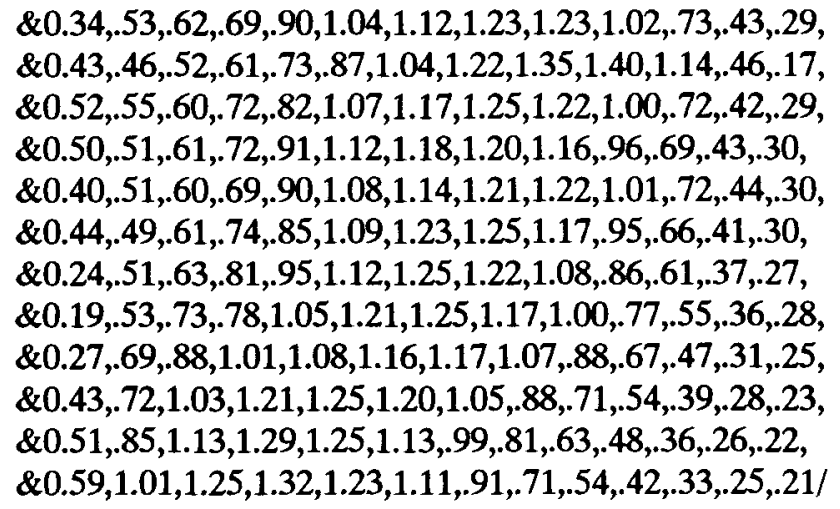

16 IF(FRACTION.GE.1.17)THEN SPENS $=0$. RETURN

ELSE IF(FRACTION.GE. 1.15 )THEN 
Appendix E

C

C FRACTION IS BETWEEN 1.15 AND 1.17

$\mathrm{C}$

SPENS $=\left(\mathrm{F}(13, \mathrm{I}-1)+\mathrm{DELE}^{*}(\mathrm{~F}(13, \mathrm{I})-\mathrm{F}(13, \mathrm{I}-1))\right)^{*}(1.17-\mathrm{FRACTION}) * 50$.

$\mathrm{SPENS}=\mathrm{SPENS} /\left(4.0^{*} 3.14159\right)$

SPENS $=$ SPENS $*$ DX90*FACT $*$ FACT

RETURN

ENDIF

$\mathrm{C}$

21 SPENS = (1.-DELE)*SLVSP2D (13,I-1,FRACTION,FX90,F,B,C,D) $\&+\quad$ DELE*SLVSP2D(13,I,FRACTION,FX90,F,B,C,D)

C

SPENS $=$ SPENS ${ }^{*}$ DX90*FACT*FACT/(4.0*3.14159)

\section{RETURN}

ENDIF

END

$$
\mathrm{C}^{*}
$$

SUBROUTINE SPLIN2D(M,IE,X,Y,B,C,D)

INTEGER N,M

REAL X(13),Y(13,20),B(13,20),C(13,20),D(13,20)

INTEGER NM1,IB,I

C

REAL T

$$
\mathrm{N}=\mathrm{M}
$$

$\mathrm{NM} 1=\mathrm{N}-1$

IF(N.LT.2) RETURN

C

IF(N.LT.3) GO TO 50

$\mathrm{D}(1, \mathrm{IE})=\mathrm{X}(2)-\mathrm{X}(1)$

$\mathrm{C}(2, \mathrm{IE})=(\mathrm{Y}(2, \mathrm{IE})-\mathrm{Y}(1, \mathrm{IE})) / \mathrm{D}(1, \mathrm{IE})$

DO $10 \mathrm{I}=2, \mathrm{NM} 1$

$\mathrm{D}(\mathrm{I}, \mathrm{IE})=\mathrm{X}(\mathrm{I}+1)-\mathrm{X}(\mathrm{I})$

$\mathrm{B}(\mathrm{I}, \mathrm{IE})=2{ }^{*}(\mathrm{D}(\mathrm{I}-1, \mathrm{IE})+\mathrm{D}(\mathrm{I}, \mathrm{IE}))$

$\mathrm{C}(\mathrm{I}+1, \mathrm{IE})=(\mathrm{Y}(\mathrm{I}+1, \mathrm{IE})-\mathrm{Y}(\mathrm{I}, \mathrm{IE})) / \mathrm{D}(\mathrm{I}, \mathrm{IE})$

$\mathrm{C}(\mathrm{I}, \mathrm{IE})=\mathrm{C}(\mathrm{I}+1, \mathrm{IE})-\mathrm{C}(\mathrm{I}, \mathrm{IE})$

10 CONTINUE

C

$\mathrm{B}(1, \mathrm{IE})=-1 .{ }^{*} \mathrm{D}(1, \mathrm{IE})$

$\mathrm{B}(\mathrm{N}, \mathrm{IE})=-1 .{ }^{*} \mathrm{D}(\mathrm{N}-1, \mathrm{IE})$

$\mathrm{C}(1, \mathrm{IE})=0$.

$\mathrm{C}(\mathrm{N}, \mathrm{IE})=0$.

IF (N.EQ.3) GO TO 15

$\mathrm{C}(1, \mathrm{IE})=\mathrm{C}(3, \mathrm{IE}) /(\mathrm{X}(4)-\mathrm{X}(2))-\mathrm{C}(2, \mathrm{IE}) /(\mathrm{X}(3)-\mathrm{X}(1))$

$\mathrm{C}(\mathrm{N}, \mathrm{IE})=\mathrm{C}(\mathrm{N}-1, \mathrm{IE}) /(\mathrm{X}(\mathrm{N})-\mathrm{X}(\mathrm{N}-2))-\mathrm{C}(\mathrm{N}-2, \mathrm{IE}) /(\mathrm{X}(\mathrm{N}-1)-\mathrm{X}(\mathrm{N}-3))$

$\mathrm{C}(1, \mathrm{IE})=\mathrm{C}(1, \mathrm{IE}) * \mathrm{D}(1, \mathrm{IE}) * * 2 /(\mathrm{X}(4)-\mathrm{X}(1))$

$\mathrm{C}(\mathrm{N}, \mathrm{IE})=-\mathrm{C}(\mathrm{N}, \mathrm{IE}) * \mathrm{D}(\mathrm{N}-1, \mathrm{IE})^{* *} 2 /(\mathrm{X}(\mathrm{N})-\mathrm{X}(\mathrm{N}-3))$ 


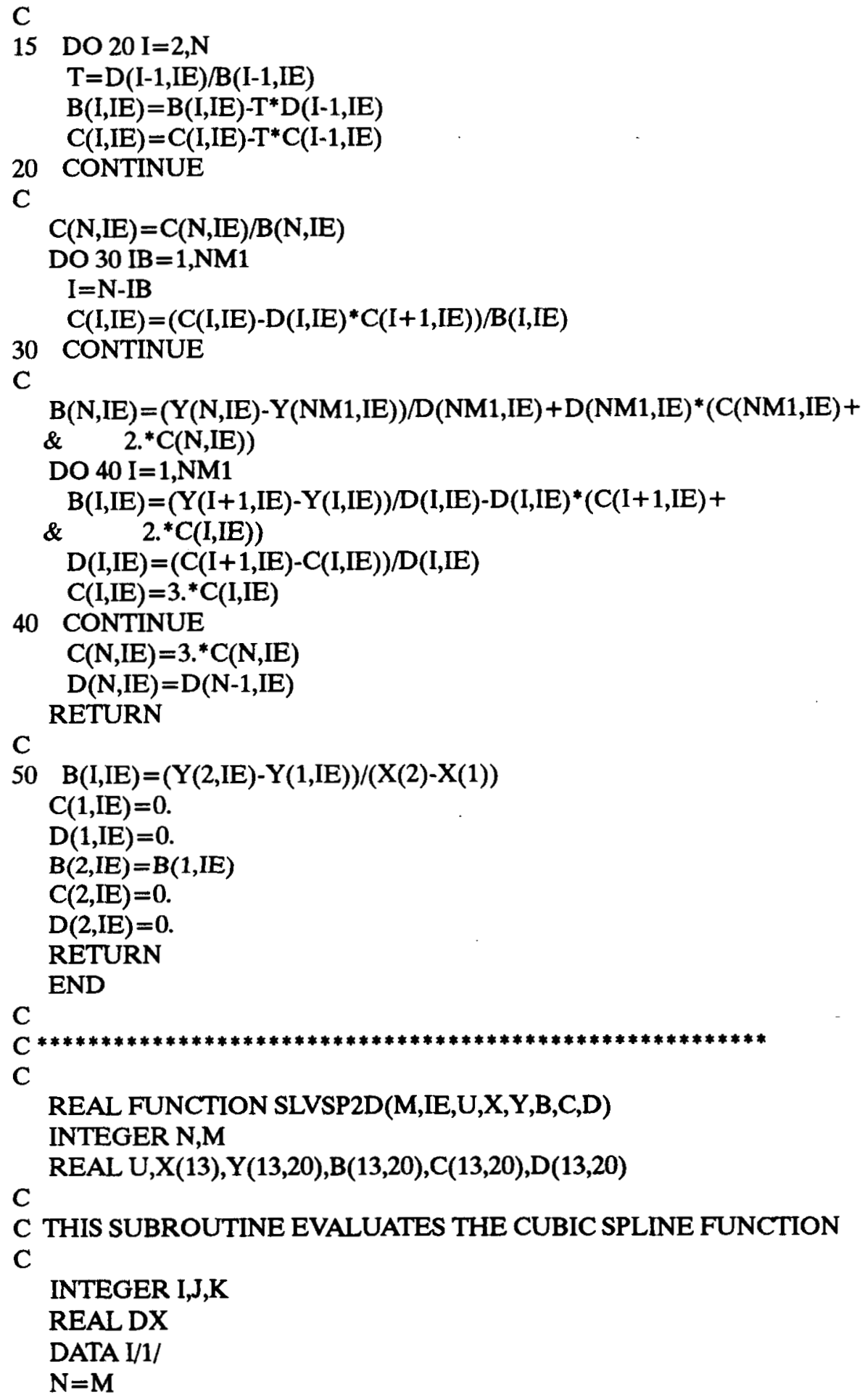


Appendix E

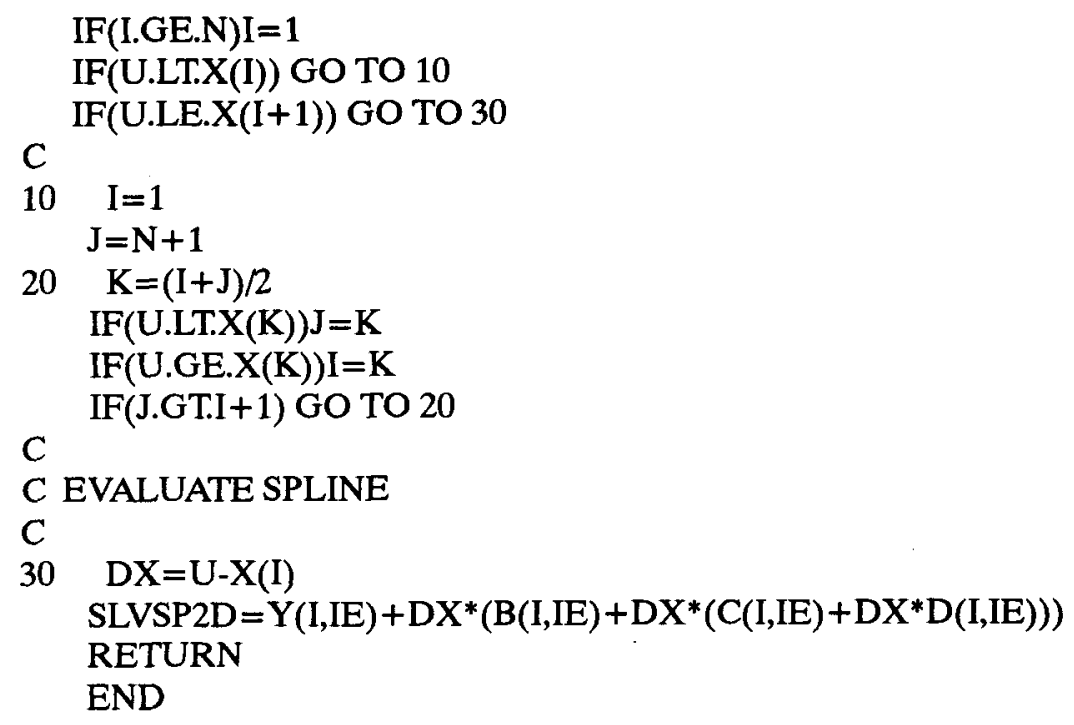




\section{DISTRIBUTION}

No. of

Copies

\section{OFFSITE}

\section{J. M. Bell}

Division of Radiation Protection \&

Emergency Preparedness

Office of Nuclear Reactor Regulation

U.S. Nuclear Regulatory Commission

Washington, DC 20555

\section{A. K Roecklein}

Radiation Protection \& Health

Office of Nuclear Regulatory Research

U.S. Nuclear Regulatory Commission

Washington, DC 20555

W. D. Reece

Texas A\&M University

Department of Nuclear Engineering, Room 129

College Station, TX 77843-3133

\section{ONSITE}

50 Pacific Northwest Laboratory

\section{G. Akabani}

J. S. Durham (39)

R. D. Stewart

W. E. Wilson

Health Physics Department Library

Publishing Coordination (2)

Technical Report Files (5)

Distr. 1 


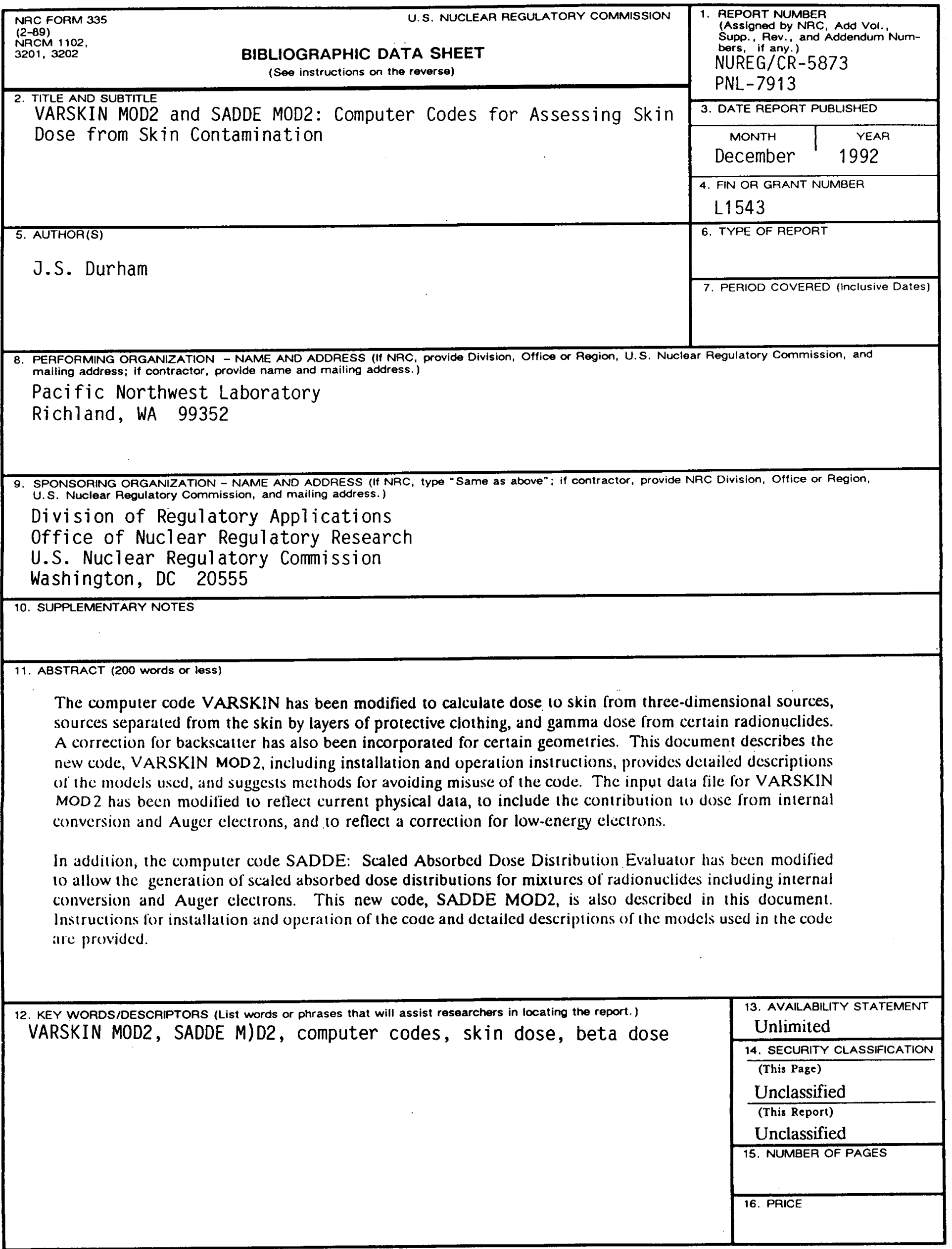

